SERVIÇO DE PÓS-GRADUAÇÃO DO ICMC-USP

Data de Depósito: 07/05/2002

Assinatura: Uourel

\title{
Categorização em Text Mining
}

\author{
Lea Silvia Martins Gonçalves
}

Orientadora: Profa. Dra. Solange Oliveira Rezende

Dissertação apresentada ao Instituto de Ciências Matemáticas e de Computação - ICMC-USP, como parte dos requisitos para obtenção do título de Mestre em Ciências de Computação e Matemática Computacional.

USP - São Carlos

Maio/2002 


\section{A Comissão Julgadora:}

Profa. Dra. Solange Oliveira Rezende

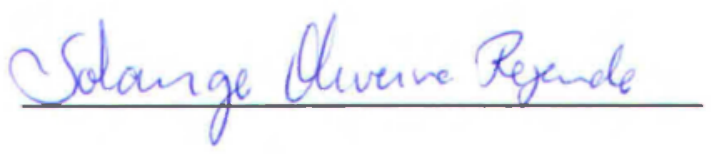

Prof. Dr. André Carlos Ponce de Leon Ferreira de Carvalho

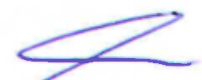

Profa. Dra. Aurora Trinidad Ramirez Pozo

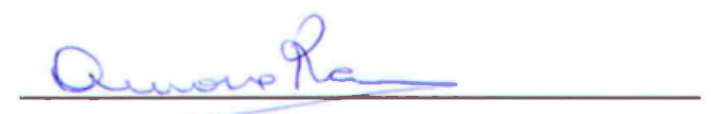


Este documento foi preparado com o formatador de textos LATEX. O sistema de citações de referências bibliográficas utiliza o padrão Chicago do sistema BibTeX.

(c) Copyright 2002 por Lea Silvia Martins Gonçalves

Todos os Direitos Reservados 


\section{Dedicatória}

Aos meus pais, Ricardo e Lourdes.

Aos meus queridos familiares Rodrigo, Larissa e Wilson. 


\section{Agradecimentos}

Tive a felicidade de contar com a colaboração de muitas pessoas nesta minha volta aos bancos escolares. Graças ao incentivo e apoio recebidos tive a satisfação de perceber em mim um gosto muito grande pelo ambiente acadêmico.

A Profa. Dra. Solange Oliveira Rezende pela orientação, dedicação, incentivo e amizade, propiciando-me engrandecimento profissional e pessoal.

À todos os professores e funcionários do ICMC-USP.

À todos os amigos do LABIC pelo companheirismo: Wálter, Valéria, Claudinha, Patrícia, Roberta, Tati, Gerson, Robson, Valmir, Adriano Pila, Flávia, João, Ronaldo; e aqueles que muito me ajudaram: Débora, Aline, Elisa, Célia e Orlando.

$\grave{A}$ amizade, carinho e ajuda da Cristiane Yae-Mi Imamura.

À Katti, Marcos Paula, Daniel e Jaqueline que auxiliaram na revisão desta dissertação.

Aos diretores do Centro de Processamento de Dados, da Escola de Engenharia de São Carlos - USP, que permitiram e apoiaram todo meu esforço na obtenção deste título, em especial, Prof. Dr. Adilson Gonzaga.

Àos amigos do Centro de Processamento de Dados, Rose Marta Marques Lourenço e Evandro Cesar Ferri Gonçalves, pelo apoio e amizade em todos os momentos.

À querida amiga e professora Helédia Calil Bueno da Costa pelo incentivo e exemplo de dignidade como ser humano.

À todos que contribuíram de um modo ou de outro, para que este trabalho pudesse ser realizado.

Agradeço a Deus pela força divina que me possibilitou a conclusão deste trabalho. 


\section{Resumo}

Os avanços tecnológicos e científicos ocorridos nas últimas décadas têm proporcionado o desenvolvimento de métodos cada vez mais eficientes para o armazenamento e processamento de dados. Através da análise e interpretação dos dados, é possível obter o conhecimento. Devido o conhecimento poder auxiliar a tomada de decisão, ele se tornou um elemento de fundamental importância para diversas organizações. Uma grande parte dos dados disponíveis hoje se encontra na forma textual, exemplo disso é o crescimento vertiginoso no que se refere à internet. Como os textos são dados não estruturados, é necessário realizar uma série de passos para transformá-los em dados estruturados para uma possível análise. O processo denominado de Text Mining é uma tecnologia emergente e visa analisar grandes coleções de documentos. Esta dissertação de mestrado aborda a utilização de diferentes técnicas e ferramentas para Text Mining. Em conjunto com o módulo de Pré-processamento de textos, projetado e implementado por Imamura (2001), essas técnicas e ferramentas podem ser utilizadas para textos em português. São explorados alguns algoritmos utilizados para extração de conhecimento de dados, "como:

Vizinho mais Próximo, Naive Bayes, Árvore de Decisão, Regras de Decisão, Tabelas de Decisão e Support Vector Machines. Para verificar o comportamento desses algoritmos para textos em português, foram realizados alguns experimentos. 


\section{Abstract}

The technological and scientific progresses that happened in the last decades have been providing the development of methods that are more and more efficient for the storage and processing of data. It is possible to obtain knowledge through the analysis and interpretation of the data. Knowledge has become an element of fundamental importance for several organizations, due to its aiding in decision making. Most of the data available today are found in textual form, an example of this is the Internet vertiginous growth. As the texts are not structured data, it is necessary to accomplish a series of steps to transform them in structured data for a possible analysis. The process entitled Text Mining is an emergent technology and aims at analyzing great collections of documents. This masters dissertation approaches the use of different techniques and tools for Text Mining, which together with the Text pre-processing module projected and implemented by Imamura (2001), can be used for texts in Portuguese. Some algorithms, used for knowledge extraction of data, such as: Nearest Neighbor, Naive Bayes, Decision Tree, Decision Rule, Decision Table and Support Vector Machines, are explored. To verify the behavior of these algorithms for texts in Portuguese, some experiments were realized. 


\section{Sumário}

1 Introdução 1

2 Uma Visão Geral de Text Mining 5

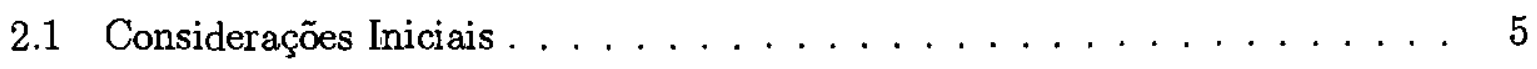

2.2 Etapas do Processo de Text Mining $\ldots \ldots \ldots \ldots \ldots$

2.2 .1 Coleta de Documentos $\ldots \ldots \ldots \ldots \ldots$

2.2 .2 Pré-processamento $\ldots \ldots \ldots \ldots \ldots \ldots$

2.2 .3 Extração de Conhecimento . . . . . . . . . . . . . 12

2.2.4 Avaliação e Interpretação dos Resultados . . . . . . . . . . . . 13

2.3 Algumas Áreas de Apoio . . . . . . . . . . . . . . . . . 17

2.3 .1 Recuperação da Informaçāo . . . . . . . . . . . . . 17

2.3.2 Processamento de Língua Natural . . . . . . . . . . . . . 18

2.3.3 Aprendizado de Máquina . . . . . . . . . . . . . . . 20

2.4 Principais Técnicas Utilizadas . . . . . . . . . . . . . . . . . . 24

2.4.1 Categorização de Textos . . . . . . . . . . . . . . . . 24

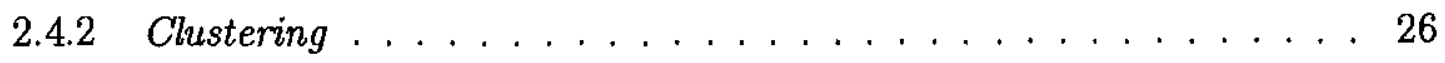

2.5 Considerações Finais $\ldots \ldots \ldots \ldots \ldots \ldots \ldots \ldots$

3 Algoritmos e Ferramentas para Categorizaçāo de Textos 28

3.1 Considerações Iniciais . . . . . . . . . . . . . . . . . . . . . 28

3.2 Alguns Algoritmos Utilizados para Text Mining . . . . . . . . . . . 30

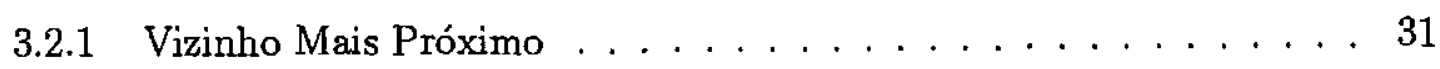

3.2 .2 Naive Bayes . . . . . . . . . . . . . . . 33

3.2.3 Algoritmos que geram Árvores de Decisão . . . . . . . . . . 34 
3.2.4 Algoritmos que geram Regras de Decisão . . . . . . . . . . . . 35

3.2.5 Redes Neurais Artificiais . . . . . . . . . . . . . . . . . 36

3.2 .6 AutoClass . . . . . . . . . . . . . . . . . 36

$3.2 .7 \quad K$-Means . . . . . . . . . . . . . . . . 38

3.2.8 Support Vector Machines . . . . . . . . . . . . . . . . . . . 39

3.3 Algumas Ferramentas Disponíveis . . . . . . . . . . . . . . . 40

$3.3 .1 \mathcal{M L C}++\ldots \ldots \ldots \ldots$. . . . . . . . . . . 40

3.3.2 Mineset ${ }^{T M} \ldots \ldots \ldots \ldots \ldots$. . . . . . . . . . . . . . . . .

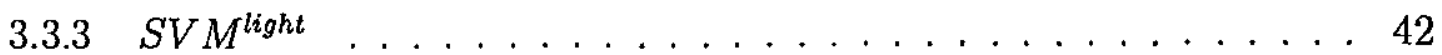

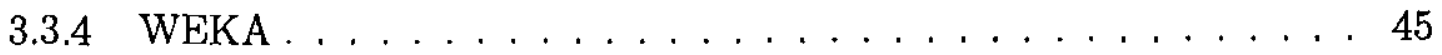

3.4 Considerações Finais . . . . . . . . . . . . . . . . 45

4 Support Vector Machines $\quad 47$

4.1 Considerações Iniciais . . . . . . . . . . . . . . . . . . . . . . . . 47

4.2 Descriçāo Geral do SVM . . . . . . . . . . . . . . . . . . 47

4.3 Teoria de Aprendizado Estatístico usado em SVM . . . . . . . . . . . 50

4.3.1 Princípio de Minimização de Risco e Convergência Uniforme . . . . 53

4.3 .2 Dimensão Vapnik-Chervonenkis . . . . . . . . . . . . . . . 59

4.3.3 Minimização de Risco Estrutural . . . . . . . . . . . . . . . . 60

4.4 Classificação usando Vetores de Suporte - Casos Lineares . . . . . . . . . 61

4.5 Generalizaçāo para Problemas não Lineares . . . . . . . . . . . . . . 65

4.6 Considerações Finais . . . . . . . . . . . . . . . . . . . . 68

$\begin{array}{lll}5 & \text { Experimentos } & 70\end{array}$

5.1 Considerações Iniciais . . . . . . . . . . . . . . . . . . . . . 70

5.2 Módulo de Pré-processamento de Textos . . . . . . . . . . . . . . . 70

5.3 Planejamento dos Experimentos . . . . . . . . . . . . . 73

5.3.1 Primeira Etapa: Coleta de Dados e Pré-processamento . . . . . . . 74

5.3.2 Segunda Etapa: Extração de Conhecimento . . . . . . . . . . 85

5.3.3 Terceira Etapa: Avaliação dos Resultados . . . . . . . . . . . . 89

5.4 Considerações Finais . . . . . . . . . . . . . . . . . . . 89 
6 Apresentação dos Resultados $\quad 90$

6.1 Considerações Iniciais . . . . . . . . . . . . . . . . . . . . . . 90

6.2 Resultados: Experimento 1 - DCan_set . . . . . . . . . . . . . . . 91

6.2.1 Sem Redução de features . . . . . . . . . . . . . . . . 91

6.2 .2 Redução para 250 features . . . . . . . . . . . . . . . . . . . . 94

6.2 .3 Redução para 500 features . . . . . . . . . . . . . . . . . 96

6.2 .4 Redução para 750 features . . . . . . . . . . . . . . . . . 101

6.2.5 Redução para 1.000 features . . . . . . . . . . . . . . . . . . . 104

6.2 .6 Redução para 1.500 features . . . . . . . . . . . . . . . . 107

6.2 .7 Redução para 3.000 features . . . . . . . . . . . . . . . 110

6.3 Resultados: Experimento 2 - DStem_set . . . . . . . . . . . . . . 112

6.3 .1 Sem Redução de features . . . . . . . . . . . . . . . . . . . . 112

6.3 .2 Redução para 250 features . . . . . . . . . . . . . . . 113

6.3.3 Redução para 500 features . . . . . . . . . . . . . . . . . 114

6.3 .4 Redução para 750 features . . . . . . . . . . . . . . 116

6.3 .5 Redução para 1.000 features . . . . . . . . . . . . . . . 117

6.3.6 Redução para 1.500 features . . . . . . . . . . . . . . . . 118

6.3 .7 Redução para 3.000 features . . . . . . . . . . . . . . . . . 120

6.4 Resultados: Experimento 3 - NCan_set . . . . . . . . . . . . . . . . . 122

6.4.1 Sem Redução de features . . . . . . . . . . . . . . . . . . . . . 122

6.4 .2 Redução para 250 features . . . . . . . . . . . . . . . . . . 123

6.4 .3 Redução para 500 features . . . . . . . . . . . . . . . . . . . . 124

6.4.4 Redução para 750 features . . . . . . . . . . . . . . . . 125

6.4 .5 Redução para 1.000 features . . . . . . . . . . . . . . . . . 126

6.4 .6 Redução para 1.500 features . . . . . . . . . . . . . . . . . 128

6.5 Resultados: Experimento 4 - NStem_set . . . . . . . . . . . . . . . 130

6.5.1 Sem Redução de features . . . . . . . . . . . . . . . . . . . . 130

6.5 .2 Redução para 250 features . . . . . . . . . . . . . . . . . 131

6.5 .3 Redução para 500 features . . . . . . . . . . . . . . . . 132

6.5.4 Redução para 750 features . . . . . . . . . . . . . . . . 135 
6.5.5 Redução para 1.000 features . . . . . . . . . . . . . . 138

6.5.6 Redução para 1.500 features . . . . . . . . . . . . . . . . . . 139

6.6 Síntese dos Experimentos . . . . . . . . . . . . . . . . . . 141

6.7 Considerações Finais . . . . . . . . . . . . . . . . . . . . 142

7 Conclusões $\quad 145$

$\begin{array}{ll}\text { Referências Bibliográficas } & 148\end{array}$ 


\section{Lista de Figuras}

2.1 Principais etapas do processo de Text Mining . . . . . . . . . . . 6

2.2 Atividades realizadas na etapa de Pré-processamento em Text Mining (Imamura 2001) ........................ 8

3.1 Esquema do processo de categorização de textos utilizando algoritmos de aprendizado de máquina . . . . . . . . . . . . . . . . . . . . 29

3.2 Representação do documento no formato bag of word . . . . . . . . . . . 30

3.3 Formato dos arquivos para entrada no $S V M^{l i g h t} \ldots \ldots \ldots$. . . . . . . . . 44

3.4 Formato dos arquivos para entrada no Weka . . . . . . . . . . . . 46

4.1 Esquema das superfícies de decisão do SVM . . . . . . . . . . . . . . 48

4.2 Dois dos muitos hiperplanos de separação (Kecman 2001) . . . . . . . . . . 49

4.3 Dependência do erro do modelo em relação ao tamanho dos dados de treinamento $($ Kecman 2001) . . . . . . . . . . . . . . . . . 50 50

4.4 Características estocásticas do aprendizado em uma coleção para um conjunto de dados de treinamento $($ Kecman 2001) . . . . . . . . . . . . . 51

4.5 Estrutura de funções de hipóteses e diferentes erros que dependem do número de funções básicas $n$, para um número fixo de exemplos $l$ (Kec$\operatorname{man} 2001) \ldots \ldots \ldots \ldots \ldots \ldots$

4.6 Dimensão VC (três pontos em $R^{2}$ separados e todos os possíveis $2^{3}=8$ $\operatorname{modos}(h=3)) \ldots \ldots \ldots \ldots . \ldots \ldots$

4.7 Hiperplano de separação ótimo (aquele que separa os dados com uma margem máxima $\ldots \ldots \ldots \ldots \ldots$. . . . . . . . . . . . 62 
5.1 Scripts que integram o módulo de Pré-processamento de textos desenvolvidos em (Imamura 2001) . . . . . . . . . . . . . . . . . 72

5.2 As etapas do processo de Text Mining no planejamento dos experimentos . 74

5.3 Principais atividades do Pré-processamento . . . . . . . . . . . . . 77

5.4 Scripts que integram o módulo de Pré-processamento e utilizados neste

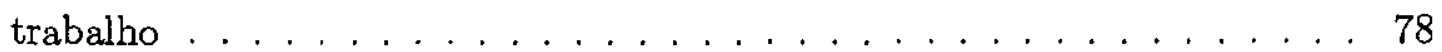

5.5 Coleta e Pré-processamento de documentos . . . . . . . . . . . . . . . 82

5.6 Segunda etapa: extração de conhecimento . . . . . . . . . . . . . . 86

5.7 Esquema para o algoritmo SVM utilizando diferentes funções Kernel . . 87

5.8 Terceira etapa: avaliação dos resultados . . . . . . . . . . . . . . . 89

6.1 Comparações dos resultados obtidos para o Erro Total com SVM e variações de parâmetros - DCan_set, sem redução de features . . . . . . . . . . 93

6.2 Comparações dos resultados obtidos (erro total) com SVM e variações de parâmetros - DCan_set, com reduçāo para 500 features . . . . . . . . . . 98

6.3 Comparaçōes dos resultados do Erro Total do SVM e os algoritmos da ferramenta Weka usando DCan_set . . . . . . . . . . . . . . . 100

6.4 Comparaçōes dos resultados obtidos (Erro Total) com SVM e variações de parâmetros com DCan_set e redução para 750 features . . . . . . . . . . 103

6.5 Comparações dos resultados obtidos (Erro Total) com SVM e variações de parâmetros, DCan_set e reduçāo para 1.000 features . . . . . . . . . . . 106

6.6 Comparações dos resultados obtidos (Erro Total) com SVM e variações de parâmetros, DCan_set e redução para 1.500 features . . . . . . . . . . . . 109

6.7 Comparações dos resultados obtidos (Erro Total) com SVM e os algoritmos da ferramenta Weka para o conjunto de dados NStem_set com redução para 500 features . . . . . . . . . . . . . . . . . 137 


\section{Lista de Tabelas}

2.1 Abordagens para atribuições de peso . . . . . . . . . . . . . . . . 10

2.2 Alguns métodos usados para seleçāo de Features em textos . . . . . . . . 12

2.3 Matriz de confusão e desempenho de classificação para duas classes . . . . 15

2.4 Cálculo das medidas de desempenho . . . . . . . . . . . . 15

2.5 Classificação dos algoritmos de AM . . . . . . . . . . . . . . . . 21

2.6 Matriz de categorização de textos . . . . . . . . . . . . . . 25

3.1 Representação de documentos, algoritmo de aprendizado usados em trabalhos relacionados . . . . . . . . . . . . . . . . . 31

5.1 Tamanho dos documentos utilizados nos experimentos . . . . . . . . 75

5.2 Exemplo da saída do algoritmo usando o etiquetador para identificação da forma canônica e stemming . . . . . . . . . . . . . . . . . . 77

5.3 Informações sobre os arquivos gerados pelos scripts de identificação de atributos . . . . . . . . . . . . . . . . . . . 79

5.4 Informaçōes sobre os arquivos gerados pelos scripts de identificação de atributos, após as reduçōes realizadas . . . . . . . . . . . . . . . . . 83

5.5 Informações sobre os arquivos gerados pelos scripts com relação aos n-grams 84

6.1 Experimento com DCan_set e algoritmo SVM, sem redução de features . . 92

6.2 Diferença em desvios-padrão com DCan_set e algoritmo SVM, sem redução de features . . . . . . . . . . . . . . . . . . . . . . 92

6.3 Experimento com DCan_set e algoritmo SVM, com redução para 250 fea-

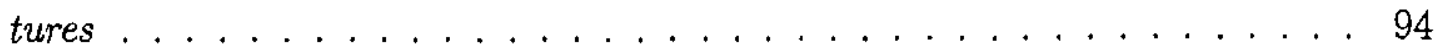


6.4 Diferença em desvios-padrão com DCan_set e algoritmo SVM, com redução para 250 features . . . . . . . . . . . . . . . . . . . . 95

6.5 Experimento com DCan_set e algoritmo SVM, com redução para 500 fea-

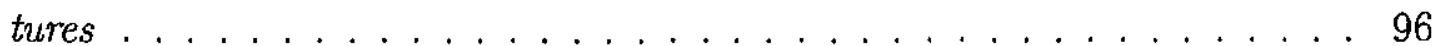

6.6 Diferença em desvios-padrão com DCan_set e algoritmo SVM, com redução para 500 features . . . . . . . . . . . . . . . . . . . . 97

6.7 Experimento com DCan_set usando a ferramenta Weka com os diferentes algoritmos e o algoritmo SVM, com redução para 500 features . . . . . . . 98

6.8 Diferença em desvios-padrão com DCan_set, algoritmo SVM e os algoritmos da Weka, com redução para 500 features . . . . . . . . . . . . . . 99

6.9 Diferença em desvios-padrão com DCan_set, algoritmo SVM e os algoritmos da Weka, com redução para 500 features . . . . . . . . . . . . . . . . . 99

6.10 Experimento com DCan_set e algoritmo SVM, com redução para 750 features . . . . . . . . . . . . . . . . . . . 101

6.11 Diferença em desvios-padrão com DCan_set e algoritmo SVM, com redução para 750 features . . . . . . . . . . . . . . . . . . . . . . . 102

6.12 Experimento com DCan_set e algoritmo SVM, com redução para 1.000 features . . . . . . . . . . . . . . . . . . . 104

6.13 Diferença em desvios-padrão com DCan_set e algoritmo SVM, com redução para 1.000 features . . . . . . . . . . . . . . . . . . . . . 105

6.14 Experimento com DCan_set e algoritmo SVM, com redução para 1.500

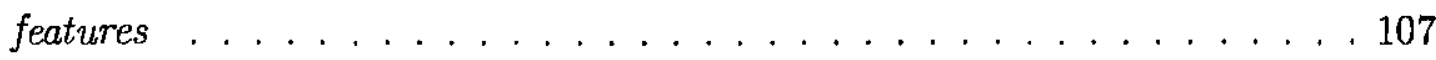

6.15 Diferença em desvios-padrão com DCan_set e algoritmo SVM, com redução para 1.500 features . . . . . . . . . . . . . . . . . . . 108

6.16 Experimento com DCan_set e algoritmo SVM, com redução para 3.000

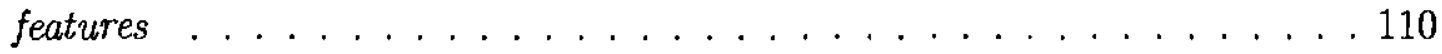

6.17 Diferença em desvios-padrão com DCan_set e algoritmo SVM, com redução para 3.000 features . . . . . . . . . . . . . . . . . . . 111

6.18 Experimento com DStem_set e algoritmo SVM, sem redução de features . 112 
6.19 Diferença em desvios-padrão com DStem_set e algoritmo SVM, sem redução de features . . . . . . . . . . . . . . . . . . . . . . . . . 113

6.20 Experimento com DStem_set e algoritmo SVM, com redução para 250

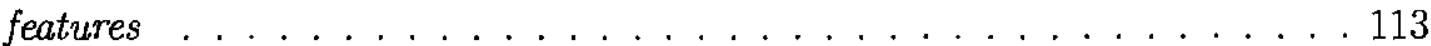

6.21 Diferença em desvios-padrão com DStem_set e algoritmo SVM, com redução para 250 features . . . . . . . . . . . . . . . . . . . . . . . 114

6.22 Experimento com DStem_set e algoritmo SVM, com redução para 500 features . . . . . . . . . . . . . . . . . . . . 115

6.23 Diferença em desvios-padrão com DStem_set e algoritmo SVM, com redução para 500 features . . . . . . . . . . . . . . . . . . . . . 116

6.24 Experimento com DStem_set e algoritmo SVM, com redução para 750

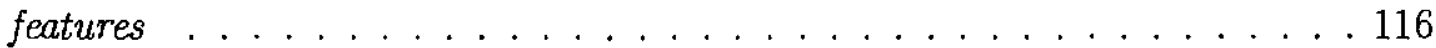

6.25 Diferença em desvios-padrão com DStem_set e algoritmo SVM, com redução para 750 features . . . . . . . . . . . . . . . . . . . . 117

6.26 Experimento com DStem_set e algoritmo SVM, com redução para 1.000 features . . . . . . . . . . . . . . . . . 118

6.27 Diferença em desvios-padrão com DStem_set e algoritmo SVM, com redução para 1.000 features . . . . . . . . . . . . . . . . . . . . . . 119

6.28 Experimento com DStem_set e algoritmo SVM, com redução para 1.500

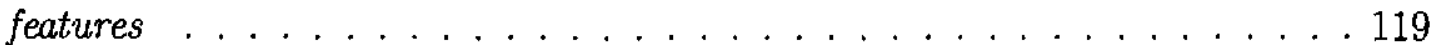

6.29 Diferença em desvios-padrão com DStem_set e algoritmo SVM, com redução para 1.500 features . . . . . . . . . . . . . . . . . . . . . . . . 120

6.30 Experimento com DStem_set e algoritmo SVM, com redução para 3.000 features . . . . . . . . . . . . . . . . . . . 120

6.31 Diferença em desvios-padrão com DStem_set e algoritmo SVM, com redução para 3.000 features . . . . . . . . . . . . . . . . . . . . . . . . 121

6.32 Experimento com NCan_set e algoritmo SVM, sem redução de features . . 122

6.33 Diferença em desvios-padrão com NCan_set e algoritmo SVM, sem redução de features . . . . . . . . . . . . . . . . . . . . . . . 123 
6.34 Experimento com NCan_set e algoritmo SVM, com reduçāo para 250 features . . . . . . . . . . . . . . . . . . . . 123

6.35 Diferença em desvios-padrão com NCan_set e algoritmo SVM, com redução para 250 features . . . . . . . . . . . . . . . . . . . . . . . . 124

6.36 Experimento com NCan_set e algoritmo SVM, com redução para 500 features . . . . . . . . . . . . . . . . . . . . . 125

6.37 Diferença em desvios-padrão com NCan_set e algoritmo SVM, com redução para 500 features . . . . . . . . . . . . . . . . . . . . 125

6.38 Experimento com NCan_set e algoritmo SVM, com redução para 750 features . . . . . . . . . . . . . . . . . . . . . 126

6.39 Diferença em desvios-padrão com NCan_set e algoritmo SVM, com redução para 750 features . . . . . . . . . . . . . . . . . . . . . . 127

6.40 Experimento com NCan_set e algoritmo SVM, com redução para 1.000 features . . . . . . . . . . . . . . . . . . 127

6.41 Diferença em desvios-padrão com NCan_set e algoritmo SVM, com redução para 1.000 features . . . . . . . . . . . . . . . . . . . . . . 128

6.42 Experimento com NCan_set e algoritmo SVM, com redução para 1.500 features . . . . . . . . . . . . . . . . . . . 128

6.43 Diferença em desvios-padrão com NCan_set e algoritmo SVM, com redução para 1.500 features . . . . . . . . . . . . . . . . . . . . . . . . . . . . 129

6.44 Experimento com NStem_set e algoritmo SVM, sem redução de features 130

6.45 Diferença em desvios-padrão com NStem_set e algoritmo SVM, sem redução de features . . . . . . . . . . . . . . . . . . . . . . . 131

6.46 Experimento com NStem_set e algoritmo SVM, com redução para 250

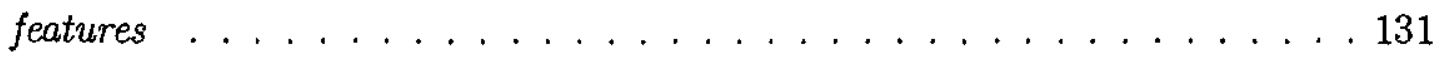

6.47 Diferença em desvios-padrão com NStem_set e algoritmo SVM, com redução para 250 features . . . . . . . . . . . . . . . . . . . . . . 132

6.48 Experimento com NStem_set e algoritmo SVM, com reduçāo para 500

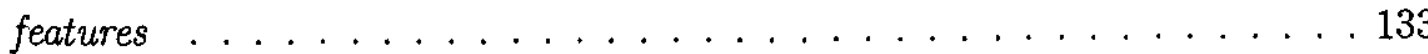


6.49 Diferença em desvios-padrão com NStem_set e algoritmo SVM, com redução para 500 features . . . . . . . . . . . . . . . . . . . . 133

6.50 Experimento com NStem_set usando a ferramenta Weka e os diferentes algoritmos e algoritmo SVM, com redução para 500 features . . . . . . . . 134

6.51 Diferença em desvios-padrão com NStem_set, algoritmo SVM e os algoritmos da Weka, com redução para 500 features . . . . . . . . . . . . 135

6.52 Diferença em desvios-padrão com NStem_set, algoritmo SVM e os algoritmos da Weka, com redução para 500 features . . . . . . . . . . . . . 136

6.53 Experimento com NStem_set e algoritmo SVM, com redução para 750

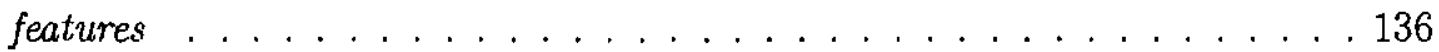

6.54 Diferença em desvios-padrão com NStem_set e algoritmo SVM, com redução para 750 features . . . . . . . . . . . . . . . . . . . 137

6.55 Experimento com NStem_set e algoritmo SVM, com redução para 1.000

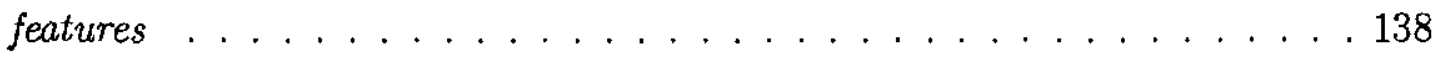

6.56 Diferença em desvios-padrão com NStem_set e algoritmo SVM, com redução para 1.000 features . . . . . . . . . . . . . . . . . . . . . . 139

6.57 Experimento com NStem_set e algoritmo SVM, com redução para 1.500

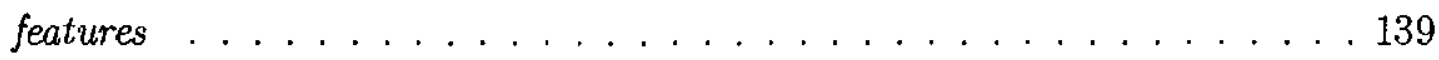

6.58 Diferença em desvios-padrão com NStem_set e algoritmo SVM, com redução para 1.500 features . . . . . . . . . . . . . . . . . . . . . 140

6.59 Erro total com algoritmo SVM, e as diferentes reduções de features . . . 141

6.60 Erro total com algoritmos da Weka, e as diferentes reduções de features . . 143 


\section{Capítulo 1}

\section{Introdução}

O advento da sociedade da informação é mais uma das grandes transformações que está sendo vivenciada nesse final de século. Passamos da era industrial, em que a capacidade de produção era o fator essencial da competitividade para um novo patamar, onde o conhecimento e a capacidade inovadora das organizações definem sua posição competitiva.

Evidentemente, o conhecimento não é o único elemento que determina se uma organização é competitiva, mas vem se tornando cada vez mais um fator decisivo na manutenção e busca de mercado. Essa importância decorre, principalmente, dos avanços tecnológicos que possibilitam acessar e armazenar uma grande gama de dados, a partir dos quais é possível inferir características de negócio de uma organização, possibilitando, por exemplo, fazer prediçōes de estratégias empregadas por seus competidores.

Um outro ponto chave para o engrandecimento do interesse em analisar tais dados é o fato de poder acessá-los e disponibilizá-los facilmente através da World Wide Web (Web ou WWW), principalmente no que se refere a dados textuais: e-mail eletrônico de clientes contendo respostas sobre produtos e serviços prestados; documentos intranets, tais como memorandos e apresentações internas; relatórios técnicos que descrevem novas tecnologias adotadas ou simplesmente em estudo; documentos e outras informações extraídos da própria Web (Tkach 1998).

Além dos textos da Web, dados textuais também são encontrados em campos de textos de base de dados, documentos eletrônicos digitalizados a partir de papéis, entre outros. Aliado ao fato de que muitas pesquisas acadêmicas têm obtido êxito com a 
aplicação do processo de Extração de Conhecimento de Base de Dados para auxiliar nas tomadas de decisões, a importância da extração de conhecimento a partir de textos, que representa cerca de $80 \%$ do conteúdo presente nos bancos de dados do mundo, vêm aumentando significativamente.

Os textos podem ser considerados, em muitos casos, como uma seqüência de dados, similares aos dados coletados por sensores ou outro sistema de informação, que podem ser processados por métodos similares aos utilizados no processo de Extração de Conhecimento de Base de Dados. A adaptação desse processo a formas textuais dá origem a um processo denominado Text Mining (Tan 1999), também conhecido como Descoberta de Conhecimento em Textos (Knowledge Discovery in Text - KDT) (Dagan 1995), ou ainda Text Data Mining (Hearst 1999). Neste trabalho será usada a nomenclatura Text Mining, que se refere à tecnologia capaz de analisar grandes coleções de documentos não estruturados com o propósito de extrair conhecimento, padrões de interesse previamente desconhecidos e potencialmente úteis.

Text Mining, tal como o processo de Extração de Conhecimento de Base de Dados, é um campo interdisciplinar, que emergiu da interseção de várias áreas, principalmente Recuperação de Informação, Aprendizado de Máquina, Estatística e Banco de Dados.

As aplicações de Text Mining podem ser úteis:

- execução de sumários, que consiste sumarizar textos, tentando apanhar as idéias mais importantes;

- clustering, que é o agrupamento de texto de acordo com semelhanças ou similaridades no conteúdo;

- identificação de idiomas;

- extração de termos;

- categorização de texto, que é classificar um documento segundo categorias (tópicos ou temas) preexistentes de documentos;

- e outras aplicações, como gestão de correio eletrônico, gestão de documentos, pesquisa e investigação de mercado, etc. 
Neste trabalho será focada principalmente categorização ${ }^{1}$ de textos, que em Text Mining, visa identificar as principais categorias em um documento, e associar esse documento a uma ou mais categorias pré-definidas (Yang \& Pedersen 1997).

A utilização de técnicas de aprendizado para categorização de textos não é uma tarefa trivial, e a etapa de aprendizado é de extrema importância, sendo a escolha da forma de representação, as escolhas das técnicas de aprendizado e suas parametrizações vitais para o desempenho do sistema.

Uma das grandes problemáticas da categorização de textos é a alta dimensionalidade dos documentos. Um documento que possui alta dimensionalidade consiste em ter centenas e até milhares de atributos. Para solucionar isso, são utilizadas técnicas de seleção de atributos, realizando operações, como remoções de atributos pouco significativos, dentre outras. O uso de técnicas de Processamento de Língua Natural se faz necessário para auxiliar na representação final dos atributos dos documentos.

Para apoiar o estudo realizado neste trabalho foram escolhidos textos em português sobre Aquisição de Conhecimento e Redes Neurais. Entretanto a maioria das ferramentas existentes para Text Mining foram desenvolvidas para o processamento de textos em inglês. Para minerar documentos em outras línguas são necessárias mudanças e adaptações das ferramentas, uma vez que mudam as características da língua (Tan 1999). Para o português muitas adaptações precisam ser realizadas, principalmente na etapa de Préprocessamento de textos.

Em (Imamura 2001), foi desenvolvido um módulo de pré-processamento de textos, que consta de scripts em Perl para a preparação dos textos em português, como: identificação de atributos e atribuições de pesos. Identificação de atributos e atribuição de pesos sāo necessários para a construção da representação textual, que normalmente é fornecida no formato atributo-valor.

Nesse contexto, este trabalho tem como objetivo principal realizar a investigação empírica de alguns algoritmos usados para extração de conhecimento de textos e prosseguir na avaliação do processo de Text Mining.

Foram investigados alguns algoritmos de aprendizado de máquina, tais como Árvores

\footnotetext{
${ }^{1}$ Também conhecido como classificação (Sebastiani 2002). Neste trabalho esses termos serão usados indistintamente.
} 
de Decisão, Regras de Decisão, Naive Bayes e principalmente, o algoritmo Support Vector Machines, considerado um dos principais algoritmos para o tratamento de bases textuais.

Para tanto, este trabalho está organizado da seguinte forma: no Capítulo 2 é feita uma abordagem sobre Text Mining, com uma breve descrição dos principais conceitos, técnicas utilizados nas aplicações de Text Mining e algumas áreas de apoio.

No Capítulo 3 é feita uma descrição de alguns dos principais algoritmos utilizados em Text Mining e algumas ferramentas que apoiaram o trabalho desenvolvido.

No Capítulo 4 é descrito o algoritmo Support Vector Machines, que vem sendo um dos mais utilizados para Text Mining, justamente pela sua alta eficiência em aplicações práticas.

No Capítulo 5 é descrito o planejamento dos experimentos e as etapas a serem realizadas. No Capítulo 6 são apresentados os resultados dos experimentos realizados, bem como uma análise dos resultados obtidos. No Capítulo 7, são apresentadas algumas considerações finais, evidenciando as linhas de pesquisa futuras e por fim as referências bibliográficas. 


\section{Capítulo 2}

\section{Uma Visão Geral de Text Mining}

\subsection{Considerações Iniciais}

O processo de Text Mining é considerado uma adaptação do processo Knowledge Discovery in Database (KDD), que vem sendo utilizado com bastante êxito em grandes bases de dados, na transformação de conhecimento implícito (embutido nos dados) em conhecimento explícito (Fayyad, Shapiro, \& Smyth 1996).

Como textos são dados não estruturados, eles precisam ser padronizados. Porém, textos normalmente aparecem em um formato difícil de ser padronizado, sendo necessário realizar um grande trabalho de pré-processamento para a utilização dos métodos de mineração de dados (Weiss \& Indurkhya 1998). Recuperação da Informação, Compreensão de Texto, Banco de Dados e Aprendizado de Máquina são áreas muito importantes dentro de Text Mining.

As técnicas mais utilizadas para o processamento textual são: categorização, clustering, sumarização e associação. O enfoque deste trabalho é a categorização de textos.

Com o objetivo de fornecer um melhor entendimento sobre algumas abordagens relacionadas ao processo Text Mining, este capítulo está dividido da seguinte forma: na Seção 2.2 são descritas as etapas desse processo. Na Seção 2.3 são apresentadas algumas áreas que o apóiam. Na seção 2.4 são descritas as principais técnicas utilizadas no processamento textual. 


\subsection{Etapas do Processo de Text Mining}

Como citado, Text Mining é um processo cuja finalidade é a extração de informações a partir de um conjunto de documentos textuais não estruturados. Esse processo pode ser dividido em quatro grandes etapas, como mostrado na Figura 2.1.

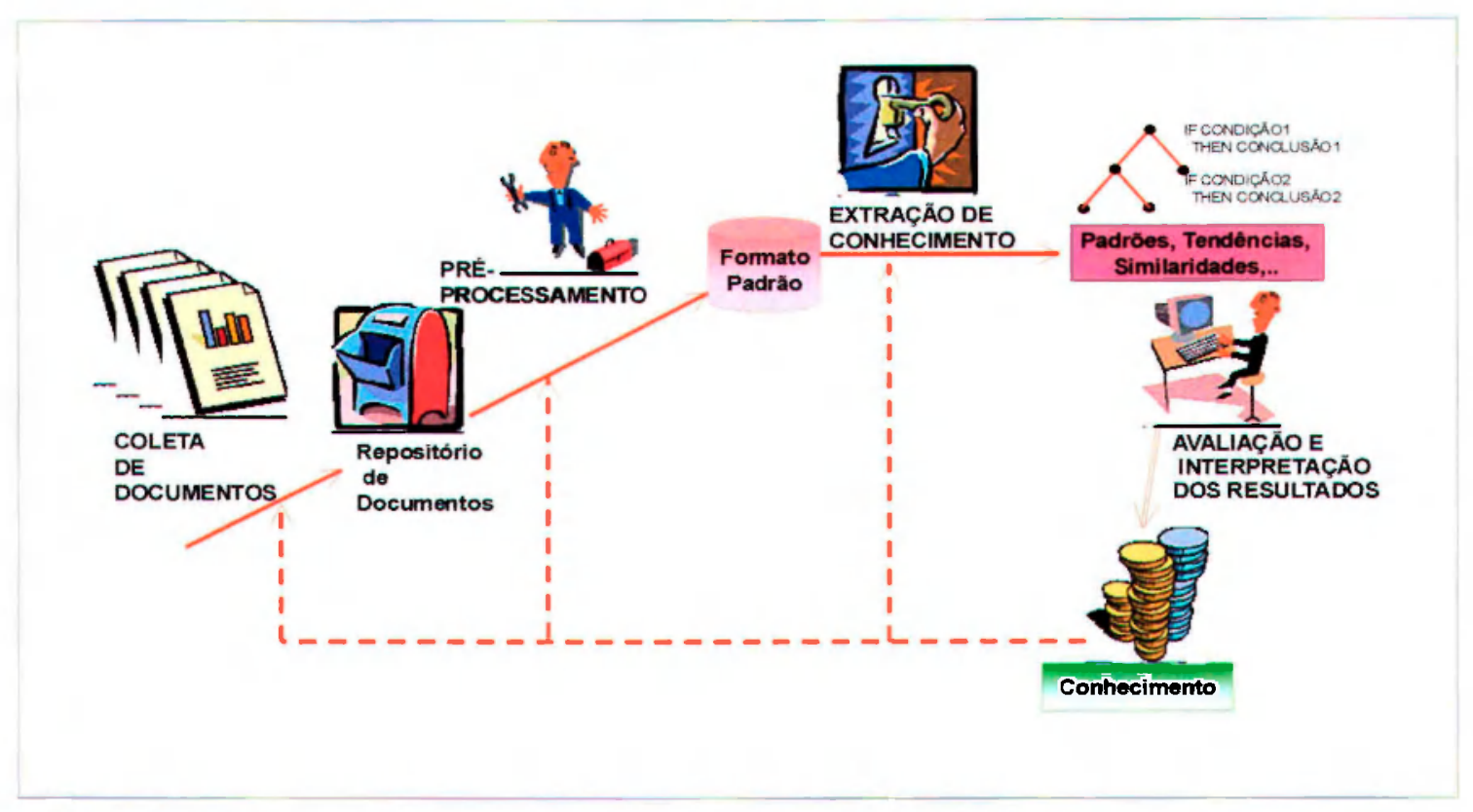

Figura 2.1: Principais etapas do processo de Text Mining

Essas etapas são descritas sucintamente a seguir:

- Coleta de Documentos: nessa etapa, os dociumentos relacionados com o domínio da aplicação final são coletados. Na maioria das vezes isso é feito automaticamente.

- Pré-processamento: consiste de um conjunto de ações realizadas sobre o conjunto de textos obtido na etapa anterior, com o objetivo de prepará-los para a extração de conhecimento. Geralmente, têm-se como resultado a padronização dos documentos em um formato atributo-valor.

- Extração de Conhecimento: nessa etapa são utilizados alguns algoritmos de aprendizado com o objetivo de extrair a partir dos documentos pré-processados, conhecimento na forma de regras de associação, relações, segmentação. classificação de textos, entre outros.

- Avaliação e Interpretação dos Resultados: nessa etapa os resultados obtidos são analisados, filtrados e selecionados para que o usuário possa ter um melhor entendimento dos textos coletados. Esse entendimento maior pode auxiliar em algum processo de tomada de decisão. 
Nas próximas subseções são descritas as abordagens e estratégias envolvidas na realização de cada etapa citada.

\subsubsection{Coleta de Documentos}

A coleta de documentos é uma etapa bastante trabalhosa. Normalmente, as ferramentas que dão suporte a essa tarefa apresentam alguns problemas, tais como:

- Falta de precisão: decorre do fato de que ainda é comum o uso de uma linguagem pouco expressiva, tornando difícil expressar qual a informação desejada; ou ainda devido ao fato de que algumas tecnologias de indexação possuem limitações no que se refere à organização de grande quantidade de documentos.

- Falta de análise inteligente: isso é ocasionado pelo fato de que não é possível verificar se determinados textos estão relacionados a algum assunto específico. Isso acontece com freqüência em documentos que são constantemente alterados, e cujas palavras chaves não cobrem os novos assuntos incluídos.

Esses problemas, entre outros, fazem com que o usuário obtenha uma lista contendo uma grande gama de documentos, muitos dos quais talvez não lhe interessam. Assim, a análise e filtragem desses documentos é uma tarefa tediosa e demorada, porém necessária.

Diante disso, técnicas utilizadas nas áreas de Recuperação da Informação, Processamento de Língua Natural e Aprendizado de Máquina vêm sendo combinadas com o intuito de melhorar a qualidade das ferramentas de busca e recuperação de documentos (Mladenić 1998).

Vale ressaltar que os documentos a serem coletados nesta etapa não são apenas aqueles disponíveis na Web, podendo ser provenientes de alguma empresa, por exemplo. Além disso, alguns campos presentes nas tabelas de bases de dados, também podem conter descrições textuais que não são interpretadas pelos métodos de extração de conhecimento de dados. Porém, esses campos podem possuir informações valiosas e, portanto, também poderiam ser considerados no processamento textual ${ }^{1}$.

\footnotetext{
${ }^{1}$ Dados textuais fazem parte da categoria de dados não-estruturados.
} 


\subsubsection{Pré-processamento}

Textos de várias origens e nos mais variados formatos podem ser coletados. No entanto, para que o processo Text Mining seja realizado, freqüentemente esses documentos são convertidos para um formato padrão (geralmente o formato $\mathrm{TXT}^{2}$ ) e armazenados em uma base de texto. Os documentos que possuem formatos diferentes são padronizados para um formato único e armazenados em uma base de textos. Dessa base de textos é extraída uma representação no formato atributo-valor que torne possível a aplicação dos algoritmos de aprendizado (Aas \& Eikvil 1999). Para realizar essa conversão, que não é uma tarefa trivial, é necessário:

- Especificar um conjunto de atributos; e

- Associar valores a esses atributos.

Essas operações podem ser identificadas, respectivamente, pela criação de uma lista de termos relevantes que pode ser auxiliada por um dicionário e pela atribuição de valores ou pesos às features ${ }^{3}$ selecionadas, gerando assim, a representação no formato atributo- valor, mostrada na Figura 2.2.

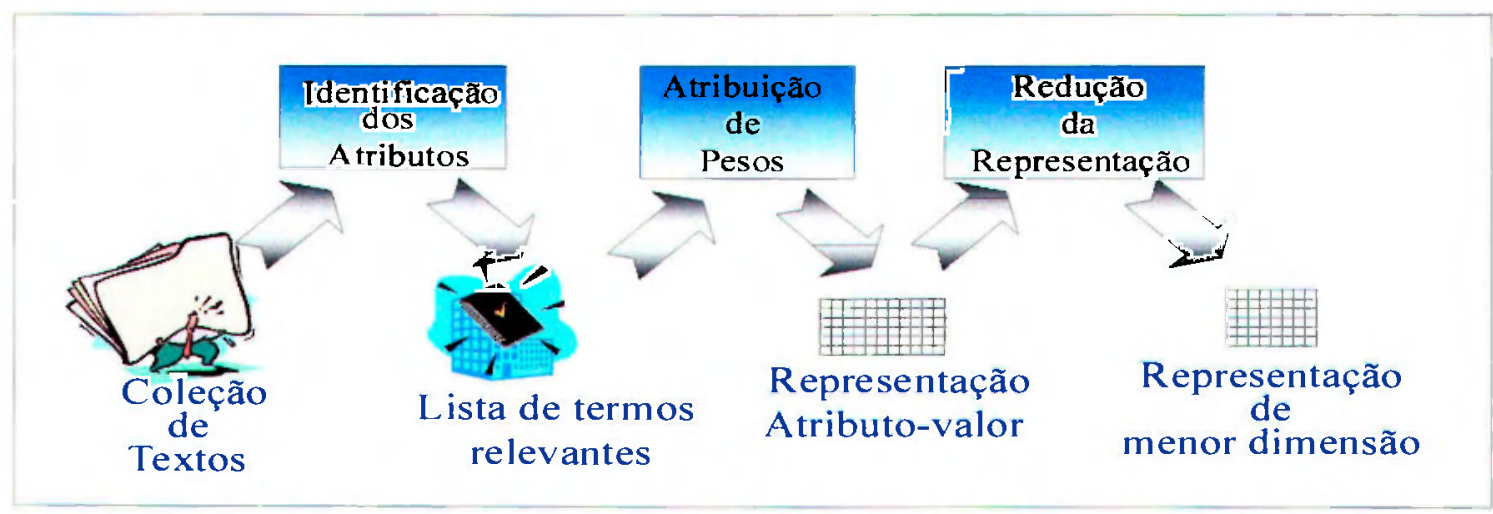

Figura 2.2: Atividades realizadas na etapa de Pré-processamento em Text Mining (Imamura 2001)

\footnotetext{
${ }^{2}$ Formato TXT refere-se a arquivos ASCII.

${ }^{3}$ Os termos features c atributos serão utilizados indistintamente neste trabalho por serem amplamente aceitos pela comunidade acadêmica da área de Inteligência Artificial.
} 
Vale ressaltar que essa representação normalmente gera arquivos com alta dimensionalidade, necessitando assim, de uma seleção de features.

O conjunto de features resultante pode utilizar dois tipos de dicionários (Weiss, Apté, Damerau, E.Johnson, \& adn Thomas Hampp 1999):

- Dicionário Universal: cuja construção envolve todos os documentos da coleção de textos, geralmente requerendo uma técnica de redução de features.

- Dicionário Local: construído considerando-se apenas os documentos de um tópico específico, e com uma quantidade fixa de termos simples selecionados de acordo com a freqüência de ocorrência na classe.

Um dicionário usualmente é composto de uma lista de palavras (features), contendo parte de todas as features do assunto abordado, relativo a língua adotada. A escolha de qual tipo de dicionário deve ser usado depende de muitos aspectos. Segundo Weiss, Apté, , Damerau, Johnson, Oles, Goetz, \& Hampp (1999), se os campos de textos ou documentos possuem tópicos altamente especializados, um dicionário universal é necessário, uma vez que a abrangência dos tópicos satisfaz a todos os documentos. Porém, se muitos tópicos distintos são cobertos, sendo que alguns deles apresentam pouca prevalência, então dicionários locais para cada tópico são mais indicados. Pode-se também fazer uso dos dicionários para identificação automática de palavras sinônimas, utilizando-se assim, de um Thesaurus.

- Para cada feature identificada deve-se associar um valor que indica o grau de relação entre a palavra e os documentos em que ela aparece.

Considerando a realização dessas duas atividades, uma coleção de documentos é representada por uma matriz $\mathbf{A}$, na qual cada entrada $a_{i k}$ representa o valor (ou peso) que é atribuído à feature $i$ com relação ao documento $k$ :

$$
A=\left(a_{i k}\right)
$$

Para calcular $a_{i k}$, algumas estratégias podem ser usadas, como peso booleano, peso pela freqüência da palavra, peso $t f^{4} \times \mathbf{i d f}^{5}$, peso $t f c^{6}$, peso $l t c^{7}$ e peso baseado em entropia.

\footnotetext{
${ }^{4} \mathrm{tf}$ (term frequency)

${ }^{5}$ idf (inverse document frequency)

${ }^{6} \mathrm{tfc}$ (term frequency component)

${ }^{7}$ Itc (logarithm term component)
} 
Na Tabela 2.1 encontram-se descritas as respectivas fórmulas usadas para calcular $a_{i k}$ seguindo cada uma dessas estratégias, de acordo com Aas \& Eikvil (1999). Nessa tabela tem-se a seguinte nomenclatura: $f_{i k}$ designa a freqüência da feature ${ }_{i}$ no documento $k, \mathbf{M}$ é o número de documentos da coleção e $\mathbf{N}$ representa o número de palavras presentes na coleção.

\begin{tabular}{|c||c|}
\hline \hline Atribuição de Peso & Fórmula Matemática \\
\hline \hline Booleano & $a_{i k}=\left\{\begin{array}{l}1, \text { se } f_{i k}>0 \\
0, \text { caso contrário }\end{array}\right.$ \\
\hline Freqüência de Palavra & $a_{i k}=f_{i k}$ \\
\hline tf $\times$ idf & $a_{i k}=f_{i k} \times \log \left(\frac{N}{n_{i}}\right)$ \\
\hline tfc & $a_{i k}=\frac{f_{i k} \times \log \left(\frac{N}{n_{i}}\right)}{\sqrt{\sum_{j=1}^{M}\left[f_{j k} \times \log \left(\frac{N}{n_{j}}\right)\right]^{2}}}$ \\
\hline Itc & $a_{i k}=\frac{\log \left(f_{i k}+1.0\right) \times \log \left(\frac{N}{n_{i}}\right)}{\sqrt{\sum_{j=1}^{M}\left[\log \left(f_{j k}+1.0\right) \times \log \left(\frac{N}{n_{j}}\right)\right]^{2}}}$ \\
\hline Entropia & $a_{i k}=\log \left(f_{i k}+1.0\right) \times\left(1+\frac{1}{\log (N)} \sum_{j=1}^{N}\left[\frac{f_{i j}}{n_{i}} \times \log \left(\frac{f_{i j}}{n_{i}}\right)\right]\right)$ \\
\hline
\end{tabular}

Tabela 2.1: Abordagens para atribuições de peso

$\mathrm{Na}$ abordagem do peso booleano, $a_{i k}$ indica a presença ou ausência da palavra no texto, enquanto que no peso pela freqüência da palavra, $a_{i k}$ refere-se ao número de ocorrências da palavra no documento. O peso tf $\times$ idf leva em consideração, além da freqüência da palavra no documento, a sua freqüência em todos os documentos da coleção. O peso tfc é semelhante a esta última abordagem, mas usa o tamanho do documento para normalizar o valor associado à feature. O peso ltc consegue reduzir o efeito das grandes diferenças de freqüência utilizando o logaritmo dos termos envolvidos na função do peso tfc. Já o peso baseado em entropia é a abordagem mais sofisticada e está centrada na idéia de informação teórica, em que leva em consideração o modo como os atributos estão distribuídos.

Muitas vezes, o tamanho da representação obtida, em relação ao número de features é grande, e conseqüentemente, pode ocorrer overfitting ${ }^{8}$, uma vez que muitas palavras podem separar as classes somente pela ocorrência randômica de suas combinações (Weiss,

\footnotetext{
${ }^{8}$ Caracteriza-se pela criação de um modelo especializado nas peculiaridades dos exemplos de treinamento, o que leva a degradação das classificações futuras e ocorre quando há um excesso de dadas para ser analisados.
} 
Apté, Damerau, Johnson, Oles, Goetz, \& Hampp 1999). Além disso, a grande quantidade de features pode levar a um processamento extremamente custoso em termos computa-. cionais e a resultados não muito confiáveis.

Para resolver os problemas de dimensão é realizada uma redução do conjunto original de features com a aplicação de duas abordagens: seleção de features e indução construtiva de features (Aas \& Eikvil 1999).

$\mathrm{Na}$ abordagem de seleção de features, tenta-se descartar as palavras menos informativas, e assim, melhorar a efetividade das técnicas de extração de padrões a serem usadas e reduzir a complexidade computacional. Com os métodos de indução construtiva de features pode-se alcançar esses objetivos por meio da combinação dos elementos da representação original para a formação de novos elementos com maior poder preditivo. Vale ressaltar que fazendo uso dos métodos de indução construtiva de features nem sempre é possível obter uma representação com número de elementos menor que a original, pois a combinação dos termos não garante a remoção dos originais.

Segundo Yang \& Pedersen (1997) existem vários métodos de seleção de features e de indução construtiva de features em textos. Quatro métodos usados em seleção de features são descritos na Tabela 2.2. Os atributos da representação original são avaliados independentemente, sendo atribuído um valor de pontuação a cada um deles. Os atributos com base nesse valor são então ordenados, e um número $n$ de atributos é considerado para formar a nova representação.

Freqüência de Documento (FD) é uma das técnicas mais simples para redução de atributos. Trata-se de uma função simples que indica a quantidade de documentos em que um atributo aparece, como valor de sua relevância.

Ganho de Informação (GI) é um critério utilizado no campo de Aprendizado de Máquina. É uma abordagem que mede o ganho de informação obtido para a predição de uma classe, tendo conhecimento da presença ou ausência de um atributo no documento. É calculado pela diferença da entropia de uma classe $c_{i}$ antes e depois do uso de um atributo conhecido.

Informação Mútua (IM) é um critério comumente utilizado em modelagem estatística de associações de palavras e aplicações relacionadas. 


\begin{tabular}{|c||c|}
\hline \hline Método & Descrição \\
\hline \hline \multirow{2}{*}{$\begin{array}{c}\text { Freqüência de Documento } \\
\text { (FD) }\end{array}$} & $\begin{array}{r}\text { Utiliza um limiar prédefinido que é comparado ao } \\
\text { número de documentos em que cada palavra } \\
\text { está presente. A palavra que possuir um valor }\end{array}$ \\
& menor que este limiar é removida \\
\hline Ganho de Informação & de uma classe por intermédio do conhecimento \\
(GI) & da presença ou ausência da palavra no documento. \\
\hline Informação Mútua & Critério usado na modelagem estatística de associações \\
(IM) & de palavras e aplicações relacionadas. \\
\hline$\chi^{2}-$ Estatístico & Mede a falta de independência entre uma feature \\
(CHI) & e uma classe \\
\hline \hline
\end{tabular}

Tabela 2.2: Alguns métodos usados para seleção de Features em textos

$\chi^{2}$ Estatístico (CHI) é uma medida estatística, que possui uma complexidade quadrática, similar a IM e GI. Trata-se de uma estratégia bastante conhecida para seleção de atributos, em que é possível medir a falta de independência entre um atributo e uma certa classe.

Yang \& Pedersen (1997) sugerem a utilização da FD ao invés dos outros métodos, por ser eficientemente semelhante aos outros, e ainda ser o método mais simples e de mais baixo custo computacional, principalmente comparado com GI e CHI, uma vez que a complexidade desses algoritmos é no mínimo de ordem quadrática. Portanto, neste trabalho optou-se pela utilização de FD.

Os métodos de redução de atributos são importantes, pois no caso de bases textuais em que palavras são normalmente indicadas como atributos, a possibilidade de se ter uma representação de grande dimensão é bastante alta.

\subsubsection{Extração de Conhecimento}

Após converter os documentos para uma representação padrão, através das técnicas já citadas, dá-se continuidade ao processo com a extração do conhecimento.

A extração de conhecimento é responsável por encontrar padrões dentro do conjunto de dados. Por se tratar de um processo interativo, pode ser necessário executar 
essa etapa diversas vezes para ajustar o conjunto de parâmetros, visando a obtençāo de resultados mais adequados aos objetivos pré-estabelecidos.

De acordo com o tipo de conhecimento almejado devem ser especificadas as tarefas de aprendizado a serem utilizadas, tais como: classificaçāo, clustering, sumarização e associação.

Decidido o tipo de tarefa a ser realizada para efetuar a busca de padrões ou modelos, é preciso escolher qual algoritmo será utilizado. Para a realização dessas tarefas podem ser empregados diversos algoritmos, sendo mais comuns os algoritmos de Aprendizado de Máquina, no caso de Categorização de textos. Porém, nenhum algoritmo é ótimo para todas as aplicações (Mitchell 1997).

Muitos estudos têm sido realizados para relacionar o algoritmo de aprendizado com a natureza do problema em questão. Uma possível solução que ainda precisa ser analisada para problemas com grande quantidade de dados, é combinar os resultados de vários algoritmos em vez de selecionar um único algoritmo. Ensembles (Breiman 1996), (Baranauskas \& Monard 2000) têm obtido muito sucesso em combinar os resultados de diferentes sistemas de aprendizado. Porém, sua utilização dificulta a fase de interpretação dos resultados e também aumenta a complexidade do modelo encontrado.

Por ser o foco principal deste trabalho, algumas técnicas e algoritmos utilizados para categorização de textos são abordadas no próximo capítulo.

\subsubsection{Avaliação e Interpretação dos Resultados}

Terminada a extração, os resultados devem ser avaliados e interpretados. Após executar o algoritmo de aprendizado e adquirir um modelo para o problema, o processo de Text Mining entra na fase de avaliação e interpretaçāo dos resultados. Nessa fase é verificado se o sistema de aprendizado atingiu as expectativas. Para isso, os resultados obtidos são avaliados por meio de algumas métricas como: taxas de erro, precisão, cobertura, entre outras.

Baseado na análise realizada nessa etapa, é determinado se os resultados obtidos são aplicáveis ao interesse originalmente proposto. Diante disso, podem ser sugeridas revisões para a formulação do problema ou até mesmo uma nova representação do problema 
analisado.

A aplicabilidade nāo é uma medida fácil de ser definida, uma vez que está diretamente relacionada com a noção de quão compreensível, válido, útil e novo é o conhecimento obtido. Um modelo pode ser considerado compreensível de acordo com sua simplicidade, porém a análise do que deve ser considerado um modelo complexo ou simples depende de muitos fatores.

Com o intuito de tornar mais simples, útil e até mesmo de facilitar a avaliação e interpretação dos padrões extraídos, muitas abordagens estatísticas e ferramentas de visualização podem ser usadas para apoiar a identificação e remoção de padrões redundantes, com pouca ou sem nenhuma utilidade para a aplicação final. Se houver necessidade de etapas anteriores serem refeitas, todo o processo será inicializado novamente, conforme ilustrado na Figura 2.1. Se não ocorreram problemas, os resultados podem ser validados e utilizados pelo usuário final em sua aplicação.

\section{Critérios de Avaliação dos Sistemas de Aprendizado}

Para um sistema de classificação de textos, as principais medidas de avaliação de desempenho são a precisão e a cobertura do sistema (Baeza-Yazte \& Ribeiro-Neto 1999). Para a tarefa de categorização de textos essas medidas devem ser calculadas para cada uma das classes consideradas.

A taxa de erro é um índice que mede mais facilmente e diretamente a performance de um sistema de aprendizado. Existem também outros critérios que podem ser utilizados para medir o desempenho do sistema de aprendizado, até mesmo com melhor eficiência do que simplesmente a taxa de erro, como velocidade, tempo de aprendizado, compreensibilidade, precisão, etc.

O desempenho de um sistema de classificação binário (ou para cada classe individual de um sistema de categorização múltiplo) é geralmente medido com o auxílio das chamadas matrizes de confusão, que oferecem uma medida da eficiência do modelo de classificação. Esse método consiste em montar uma matriz, onde é identificado o número de elementos classificados corretamente e incorretamente para cada classe, como mostrado na Tabela 2.3. 
Com base na matriz de confusão, é possível calcular: a precisão, a cobertura e os graus de falhas e acerto.

Pode-se também calcular a taxa de erro, que é uma dedida bastante utilizada para avaliar o desempenho de sistemas de aprendizado. Algumas taxas de erro são: erro total (Yang 1999), erro na classe positiva $C_{+}$e erro na classe negativa $C_{-}$(Mitchell 1997). Essas medidas estão mostradas na Tabela 2.4.

\begin{tabular}{|c||cc||cc|}
\hline \hline $\begin{array}{c}\text { Rótulo } \\
\text { da Classe }\end{array}$ & $\begin{array}{c}\text { Predito } \\
\text { como } C_{+}\end{array}$ & $\begin{array}{c}\text { Predito } \\
\text { como } C_{-}\end{array}$ & $\begin{array}{c}\text { Taxa de Erro } \\
\text { da Classe }\end{array}$ & $\begin{array}{c}\text { Taxa de Erro } \\
\text { Total }\end{array}$ \\
\hline \hline$C_{+}$ & $T_{p}$ & $F_{N}$ & $\frac{F_{N}}{T_{P}+F_{N}}$ & $\frac{F_{P}+F_{N}}{n}$ \\
$C_{-}$ & $F_{P}$ & $T_{N}$ & $\frac{F_{P}}{F_{P}+T_{N}}$ & \\
\hline
\end{tabular}

Tabela 2.3: Matriz de confusão e desempenho de classificação para duas classes

\begin{tabular}{|c|c|}
\hline \hline Critérios & Fórmula \\
\hline \hline Precisão & $\frac{T_{P}}{T_{P}+F_{N}}, T_{P}+F_{N}>0$ \\
\hline Cobertura (recall) & $\frac{T_{P}}{T_{P}+F_{P}}, T_{P}+F_{P}>0$ \\
\hline Grau de Falha (fallout) & $\frac{F_{N}}{F_{N}+T_{N}}, F_{N}+T_{N}>0$ \\
\hline Grau de Acerto (accuracy) & $\frac{T_{P}+T_{N}}{n}, n>0$ \\
\hline Erro Total & $\frac{F_{N}+F_{P}}{n}, n>0$ \\
\hline Erro em $C_{+}$ & $\frac{F_{N}}{T_{P}+F_{N}}, T_{P}+F_{N}>0$ \\
\hline Erro em $C_{-}$ & $\frac{F_{P}}{F_{P}+T_{N}}, F_{P}+T_{N}>0$ \\
\hline \hline
\end{tabular}

Tabela 2.4: Cálculo das medidas de desempenho

onde: total de instâncias $=\mathrm{n}=T_{P}+F_{N}+F_{P}+T_{N}$

$T_{P}=$ número de exemplos positivos classificados corretamente, ou verdadeiros positivos

$F_{N}=$ número de exemplos positivas classificadas de forma errada, ou falsos negativos

$F_{P}=$ número de exemplos negativos classificados de forma errada, ou falsos positivos

$T_{N}=$ número de exemplos negativas classificadas corretamente, ou verdadeiros negativos

Uma medida alternativa bastante difundida é a $E_{\text {measure }}$ que combina os valores de cobertura e precisão numa única fórmula. Essa medida nada mais é do que a média harmônica, favorecendo sistemas que tenham valores similares em sua precisão e cobertura (Rijsbergen 1979) apud (Lewis \& Gale 1994), cuja fórmula é apresentada na equação 2.2. 


$$
E_{\beta}=1-\frac{\left(\beta^{2}+1\right) \text { Precisao } * \text { Cobertura }}{\beta^{2} * \text { Precisao }+ \text { Cobertura }}
$$

onde $\beta$ é um parâmetro definido pelo usuário, entre 0 e infinito, e controla o peso relativo entre precisão e cobertura.

Para $\beta=1$, corresponde a igualar em importância a precisão e a cobertura, onde se define $F_{\beta=1}=1-E_{\beta=1}$. Para Text Mining $F_{1}$ é uma medida bastante utilizada (Yang \& Liu 1999), cuja fórmula é dada na equação 2.3

$$
F_{1}=\frac{2 * \text { Precisao } * \text { Cobertura }}{\text { Precisao }+ \text { Cobertura }}
$$

Outra medida utilizada é a diferença em desvio-padrão do erro. Suponha que se queira comparar dois algoritmos com taxas de erro iguais a $9,00 \pm 1,00$ e 7,50 $\pm 0,80$. Qual dessas taxas é a melhor? Será que existe uma diferença significativa de um para o outro com relação às taxas de erro apresentadas por eles, com nível de confiança de $95 \%$ ?

Para responder essa pergunta, assume-se o caso geral para determinar se a diferença de um algoritmo para outro, por exemplo $A_{1}$ e $A_{2}$, é significativa ou nāo. Combina-se a média e o desvio padrão, conforme as Equações 2.4 e 2.5, respectivamente. Já a diferença absoluta em desvio-padrão é calculada através da Equação 2.6 (Weiss \& Indurkhya 1998) e (Baranauskas \& Monard 2000).

$$
\begin{gathered}
\operatorname{mean}\left(A_{2}-A_{1}\right)=\operatorname{mean}\left(A_{2}\right)-\operatorname{mean}\left(A_{1}\right) \\
\operatorname{sd}\left(A_{2}-A_{1}\right)=\sqrt{\frac{s d\left(A_{2}\right)^{2}+\operatorname{sd}\left(A_{1}\right)^{2}}{2}} \\
\operatorname{ad}\left(A_{2}-A_{1}\right)=\frac{\operatorname{mean}\left(A_{2}-A_{1}\right)}{\operatorname{sd}\left(A_{2}-A_{1}\right)}
\end{gathered}
$$

Assumindo uma distribuição normal, se $a d\left(A_{2}-A_{1}\right)>0$, pode-se dizer que $A_{1}$ tem um desempenho melhor que $A_{2}$. Se $a d\left(A_{2}-A_{1}\right) \geq 2$ desvios-padrão, pode-se dizer que $A_{1}$ tem melhor desempenho que $A_{2}$ com nivel de confiança de $95 \%$. Por outro lado, se ad $\left(A_{2}-A_{1}\right)<0$ diz-se que $A_{2}$ tem um desempenho melhor que $A_{1}$ e se ad $\left(A_{2}-A_{1}\right) \geq-2$ desvios-padrāo, então $A_{2}$ tem um desempenho melhor que $A_{1}$ com nível de confiança de 95\% (Baranauskas \& Monard 2000). 
Retornando ao exemplo descrito anteriormente, em que $A_{2}=9,00 \pm 1,00$ e $A_{1}=$ $7,50 \pm 0,80$. Baseado apenas nos números, há uma tendência em dizer que $A_{1}$ é melhor que $A_{2}$, mas usando as Equações 2.4, 2.5 e 2.6, obtém-se os seguintes resultados:

$$
\begin{gathered}
\operatorname{mean}\left(A_{2}-A_{1}\right)=9,00-7,50=1,50 \\
\operatorname{sd}\left(A_{2}-A_{1}\right)=\sqrt{\frac{1,00^{2}+0,80^{2}}{2}}=0,91 \\
\operatorname{ad}\left(A_{2}-A_{1}\right)=\frac{1,50}{0,91}=1,65
\end{gathered}
$$

Conseqüentemente, como $a d\left(A_{2}-A_{1}\right) \geq 0$, então $A_{2}$ tem um desempenho melhor que $A_{1}$, e como ad $\left(A_{2}-A_{1}\right)$ não é $\geq 2$, não pode-se dizer que $A_{2}$ tem um desempenho melhor que $A_{1}$, com grau de confiança de $95 \%$.

\subsection{Algumas Áreas de Apoio}

Text Mining tem buscado a integração de diversas áreas para obter melhores resultados. Dentre essas áreas destacam-se: Recuperação da Informação, Processamento de Língua Natural e Aprendizado de Máquina.

\subsubsection{Recuperação da Informação}

A Recuperação da Informação (RI) engloba atualmente qualquer tarefa que tenha por objetivo representar, organizar, armazenar, indexar e prover acesso a itens de informação (Baeza-Yazte \& Ribeiro-Neto 1999).

Um item de informação é geralmente constituído de texto (tais como documentos diversos, páginas Web, livros), embora possa conter outros tipos de dados tais como fotografias, gráficos e figuras. A representação e organização dos itens de informação devem fornecer ao usuário acesso facilitado às informações de seu interesse.

A área de RI cresceu muito em importância com a introdução da Web. A despeito de seu sucesso, a Web introduziu uma série de problemas. De fato, encontrar informação útil na Web é freqüentemente uma tarefa tediosa e difícil, devido à grande quantidade de 
dados disponíveis. A maior dificuldade é a ausência de um modelo de dados claramente definido. Isso implica que a semântica e a estrutura dos dados é freqüentemente mal definida e de baixa qualidade. Tais dificuldades têm atraído a atenção para a área de RI e suas técnicas.

Recuperação da Informação é uma das áreas ligadas ao Text Mining, principalmente no que diz respeito à etapa de coleta de documentos, na qual há necessidade de se coletar textos relacionados a um domínio específico. A recuperação de textos, palavras e frases chaves são comumente usadas para auxiliar o processo de pesquisa e fazer com que esse ocorra mais rapidamente e com um melhor grau de eficiência.

Alguns trabalhos relacionados a essa área são (Crestani, Lalmas, Rijsbergen, \& Campbell 1998), (Belkin \& Croft 1992) e (Cooper 1995).

\subsubsection{Processamento de Língua Natural}

A pesquisa em Processamento de Língua Natural (PLN) vem se tornando cada vez mais importante devido à diversas razões, como por exemplo, as novas aplicaçōes que surgiram com o advento da Web.

Buscar as informações relevantes e evitar aquelas que não o são é uma tarefa que só pode ser realizada com sucesso por programas com uma boa capacidade de compreensão de língua natural, ainda que parcial. Na maioria dos casos é necessário, mais do que indexar um texto recorrendo a lemas, é preciso representar as relações que esses textos encerram e que de um modo geral estão implícitas.

A ambigüidade é, possivelmente, um dos maiores problemas no Processamento de Língua Natural. O português é uma língua natural cheia de ambigüidades e, nesse aspecto, difere radicalmente das linguagens formais. Um outro problema é como representar o significado, ou significados, de uma determinada forma lingüística que consigamos representar. Além desses problemas, também podem ser citados:

- As frases de uma língua são descriçōes incompletas das informações que pretendem transmitir;

- A mesma expressão pode ter significados distintos em diferentes contextos; 
- Nenhum programa de Língua Natural pode ser completo. Isso porque novas palavras, expressões e significados podem ser gerados com bastante liberdade;

- Há inúmeras maneiras de expressar um mesmo pensamento.

Para resolver tais problemas, PLN conta com o processo de análise. As principais etapas desse processo são:

- Análise Fonética: reconhecimento das sílabas a partir dos sons que formam o enunciado.

- Análise Morfológica: identificação dos elementos básicos que formam as palavras. É o estudo da estrutura e da classificação das palavras em função do uso: substantivo, adjetivo, pronome, verbo, numeral, artigo, advérbio, preposiçāo, conjunção. Por exemplo: o verbo chegou expressa a ação de chegar mas também, devido à presença do sufixo -ou", temos a informaçāo que essa ação aconteceu no passado. Outro exemplo: a palavra pessoas contém o sufixo -s"que indica que a palavra denota mais de uma pessoa.

- Análise Sintática: o resultado da análise morfológica é um conjunto de palavras cujas classes foram identificadas, junto com todas as informações pertinentes que foi possível extrair da composiçāo morfológica. A análise sintática tenta identificar como as palavras se combinam para determinar a estrutura da frase.

- Análise Semântica: durante a análise semântica, utiliza-se a estrutura gerada durante a análise sintática para construir outras estruturas que representem o significado das sentenças (redes semânticas, frames, etc.).

- Análise do Discurso: para interpretar uma frase, às vezes é preciso se referir às frases anteriores. É o caso dos pronomes. Considere, por exemplo, a segunda frase do seguinte discurso: Há muito tempo que João está com meu livro. Eu gostaria de recuperá-lo. Para interpretar o pronome da segunda frase, é preciso procurar um antecedente na frase que precede. Nesse caso, temos um mecanismo para relacionar o pronome com meu livro e não com João. 
- Análise Pragmática: diz respeito à procura de sentido nos sistemas de signos, considerando o contexto, os costumes e usos lingüísticos dos falantes e as regras sociais. Impõe-se, portanto: quem disse, em que circunstâncias, e com que intenções, isto é, o uso que os interlocutores fazem sobre a linguagem tendo em vista a ação que exercem uns sobre os outros.

Os métodos de Processamento de Língua Natural são considerados importantes na preparação dos dados para obter uma representação mais consistente em relação ao conteúdo dos documentos.

\subsubsection{Aprendizado de Máquina}

Aprendizado de Máquina (AM) é uma das sub-áreas de Inteligência Artificial que pesquisa métodos computacionais relacionados à aquisição de novos conhecimentos, novas habilidades e novas formas de organizar conhecimento já existente (Michalski, Bratko, \& Kubat 1998).

Entre os principais objetivos de AM estão:

- Melhor entendimento dos mecanismos de aprendizado humano;

- Automação da aquisição do conhecimento;

- Aquisição de novos conhecimentos.

A escolha de um algoritmo de Aprendizado de Máquina não é uma tarefa trivial, levando-se em consideração que é necessária a verificação do tamanho da base dados, ou de textos, do domínio da aplicação, da precisão exigida, entre outros. Os estudos na área de Aprendizado de Máquina devem ser realizados levando-se em conta os objetivos a serem alcançados e de preferência, contar com um especialista do domínio.

Classificar os algoritmos de Aprendizado de Máquina também não é uma tarefa fácil, pois existe uma infinidade de critérios para agrupá-los. Os algoritmos de Aprendizado de Máquina podem ser classificados de acordo com vários aspectos: por tipo de aprendizado, por tipo de paradigma, por tipo de linguagem de descrição e pelo modo de incorporação de novos exemplos, conforme mostrado na Tabela 2.5. Nessa tabela é 
apresentado um resumo das características gerais para classificação dos algoritmos de Aprendizado de Máquina.

\begin{tabular}{|c|c|c|c|}
\hline $\begin{array}{c}\text { Tipo de } \\
\text { Aprendizado }\end{array}$ & $\begin{array}{l}\text { Paradigmas de } \\
\text { Aprendizado }\end{array}$ & $\begin{array}{l}\text { Linguagens de } \\
\text { Descriçāo }\end{array}$ & $\begin{array}{c}\text { Modo que os } \\
\text { Novos Exemplos } \\
\text { säo Integrados }\end{array}$ \\
\hline $\begin{array}{c}\text { - Supervisionado } \\
\text { - Nāo Supervisionado }\end{array}$ & $\begin{array}{l}\text { - Simbólico } \\
\text { - Estatístico } \\
\text { - Instance-Based } \\
\text { - Conexionista } \\
\text { - Genético }\end{array}$ & $\begin{array}{c}\text { - Exemplo } \\
\text { - Hipóteses ou } \\
\text { Conceitos Aprendidos } \\
\text { - Teoria de Domínio } \\
\text { ou Conhecimento de } \\
\text { Fundo }\end{array}$ & $\begin{array}{l}\text { - Incremental } \\
\text { - Não Incremental }\end{array}$ \\
\hline
\end{tabular}

Tabela 2.5: Classificação dos algoritmos de AM

Com relação ao tipo de aprendizado, isto é, de acordo como eles aprendem, podem ser classificados em:

- Aprendizado Supervisionado: também conhecido como aprendizado dirigido, no qual um agente externo (que poderá ser um especialista do domínio) apóia a preparação dos dados, definindo as classes ou valores contínuos (ou numéricos), alimentando com exemplos de cada classe e verificando os resultados obtidos. Existe um atributo-meta e de acordo com os valores que ele assume, a tarefa é denominada classificação ou regressão. O sistema deve encontrar modelos capazes de classificar novos exemplos cuja classe é desconhecida. Alguns algoritmos de aprendizado supervisionado são: Naive Bayes, CN2, C4.5 e FOIL (algoritmos para classificação) e RT, Cubist, CART, FORS (algoritmos para regressão).

- Aprendizado Não Supervisionado: também conhecido como aprendizado por observação e descoberta. O conjunto de dados não apresenta um atributo-meta, isto é, não possui um atributo cujo valor deve ser predito para novos exemplos. Nesse caso, agrupamentos são encontrados a partir de alguma caracterização lógica de regularidade, sem ajuda de um especialista do domínio. Um conjunto de exemplos é 
fornecido ao sistema a fim de que seja estabelecida a existência de clusters (agrupamentos) ou até mesmo agregações desses dados. Alguns algoritmos são: AutoClass, K-Means e $k$ Vizinho Mais Próximo.

Com relação ao tipo de paradigma eles podem ser classificados como:

- Simbólico: explora representações de estruturas gráficas ou lógicas. Os sistemas de aprendizado simbólico tentam aprender construindo representações simbólicas de um conceito por meio da análise de exemplos e contra exemplos desse conceito. $O$ conhecimento obtido é representado por alguma expressão lógica, rede semântica, regras de produção, árvores de decisāo ou árvores de regressão (Baranauskas \& Monard 2000). Os métodos de aprendizado simbólico podem ser tratados como: proposicional e relacional, de acordo com o tipo de lógica que utilizam para representar o conceito aprendido (Tecuci \& Kodratoff 1995).

- Instance-Based: uma forma de classificar um caso é utilizar de um caso similar cuja classe é conhecida e assumir que o novo caso terá a mesma classe. Essa filosofia exemplifica os sistemas instance-based, que classificam casos nunca vistos por intermédio de casos similares conhecidos (Quinlan 1993). Resultados dessa abordagem também podem ser encontrados em (Yang 1999).

- Estatístico: técnicas estatísticas tendem a focar tarefas em que todos os atributos possuem valores contínuos ou ordinais. Muitos podem ser também paramétricos, assumindo alguma forma de modelo, e então, encontrando valores apropriados para os parâmetros, a partir dos dados. Os métodos estatísticos estão sendo empregados principalmente em categorização, onde os valores de atributos podem estar normalmente distribuídos (Aas \& Eikvil 1999).

- Conexionista: Redes Neurais Artificiais (RNA) são modelos matemáticos que foram inspiradas no modelo biológico do sistema nervoso. Sua representação envolve unidades altamente interconectadas (o que gerou o nome conexionismo). As RNAs possuem capacidade de aprender por exemplos e fazer interpolações e extrapolações do que aprendem. No paradigma conexionista é necessário determinar 
o peso das conexões entre as unidades (Braga, Carvalho, \& Ludermir 2000). Resultados sobre o uso de redes neurais em Text Mining e categorização de textos podem ser encontrados em (Lagus 2000).

- Genético: esse formalismo de classificação é derivado do modelo evolucionário de aprendizado. Um classificador genético consiste de uma população de elementos de classificação que competem para fazer a predição. Elementos que possuem uma performance fraca são descartados, enquanto os elementos mais fortes proliferam, produzindo variações de si mesmo. Esse paradigma possui uma analogia direta com a teoria de Darwin, onde sobrevivem os mais bem adaptados ao ambiente. Os Algoritmos Genéticos trabalham com um conjunto de soluções simultaneamente. Algoritmos para classificação de textos combinados com algoritmos genéticos foram utilizados para fazer uma predição sobre dados financeiros em (Thomas \& Sycara 1999).

Em um sistema de aprendizado é necessário utilizar linguagens para representar os exemplos e conceitos induzidos. Essas linguagens são comumente referenciadas como linguagens de descrição e podem ser classificados como :

- Exemplos: descreve o formato dos exemplos utilizados pelo algoritmo de AM para aprender conceitos, estabelecendo limites sobre os tipos de padrões que o sistema pode aprender.

- Hipóteses ou Conceitos Aprendidos: descreve o estado interno de um algoritmo de Aprendizado de Máquina, correspondente à teoria dos conceitos ou padrões que existem nos dados, estabelecendo limites sobre o que pode ou não pode ser aprendido.

- Teoria de Domínio ou Conhecimento de Fundo: descreve todo o conhecimento prévio que o sistema de aprendizado possui a respeito do domínio.

Os algoritmos de aprendizado indutivo podem ser classificados de duas formas, segundo o modo que integram novos exemplos: 
- Incremental: revê a definição do conceito atual, se necessário, em resposta a cada novo exemplo de treinamento observado. Os exemplos observados são considerados um a um pelo sistema, isto é, o sistema considera o primeiro exemplo e, de acordo com esse exemplo, constrói uma determinada hipótese. A seguir considera um segundo exemplo, que pode ou não modificar a primeira hipótese, baseando-se em como essa classifica o segundo exemplo. A medida em que mais exemplos são apresentados, o sistema continua modificando o conceito.

- Não Incremental: necessita que todos os exemplos de treinamento estejam disponíveis simultaneamente para que seja induzido um conceito. É vantajoso usar esses algoritmos para problemas de aprendizado onde todos os exemplos estão disponíveis e, provavelmente, não irão ocorrer mudanças.

\subsection{Principais Técnicas Utilizadas}

Com relação ao processamento textual, as técnicas mais citadas são: categorização, clustering, sumarização e associação. Neste trabalho será dada ênfase somente na categorização e clustering de textos.

\subsubsection{Categorização de Textos}

Categorização de textos é o processo de agrupar documentos dentro de diferentes categorias ou classes pré-definidas (Joachims 1997), e como mencionado anteriormente, e trata-se de um problema de aprendizado supervisionado.

Segundo Sebastiani (2002), categorização de textos pode ser definida como a tarefa de gerar um modelo de classificação com base numa matriz como mostrado na tabela 2.4.1, em que cada tupla contém informações sobre um documento $d_{i}, i=[1, m]$. Essas informações compreendem o peso, definido como $a_{i j}$, que cada termo termo $o_{j}, j=[1, n]$, tem em relação a cada documento $d_{i}$. Cada documento $d_{i}$ tem adicionado ainda a informação sobre sua respectiva classe $c_{k}$.

A categorização de textos pode ser utilizada em muitos contextos, desde indexação automática de documentos baseada em um vocabulário de controle, filtragem de docu- 


\begin{tabular}{|c||c|c|c|c|c|c|c|c|}
\hline \hline documento & termo $_{1}$ & $\ldots$ & $\ldots$ & termo $_{j}$ & $\ldots$ & $\ldots$ & termo & Classe \\
\hline \hline$d_{1}$ & $a_{11}$ & $\ldots$ & $\ldots$ & $a_{1 j}$ & $\ldots$ & $\ldots$ & $a_{1 n}$ & $c_{k}$ \\
\hline$\ldots$ & $\ldots$ & $\ldots$ & $\ldots$ & $\ldots$ & $\ldots$ & $\ldots$ & $\ldots$ & $\ldots$ \\
\hline$d_{i}$ & $a_{i 1}$ & $\ldots$ & $\ldots$ & $a_{i j}$ & $\ldots$ & $\ldots$ & $a_{i n}$ & $c_{k}$ \\
\hline$\ldots$ & $\ldots$ & $\ldots$ & $\ldots$ & $\ldots$ & $\ldots$ & $\ldots$ & $\ldots$ & $\ldots$ \\
\hline$d_{m}$ & $a_{m 1}$ & $\ldots$ & $\ldots$ & $a_{m j}$ & $\ldots$ & $\ldots$ & $a_{m n}$ & $c_{k}$ \\
\hline \hline
\end{tabular}

Tabela 2.6: Matriz de categorização de textos

mentos, generalização automática de meta-dados, catálogos de recursos na Web e, em geral, qualquer aplicação que requeira organização de documentos ou seleção de mensagens (Sebastiani 2002).

Até a década de 80 a maioria dos trabalhos em categorização de textos era referente à construção automática de sistemas de categorização usando técnicas de engenharia de conhecimento, isto é, manualmente definia-se o conjunto de regras lógicas que codificava conhecimento especialista relacionado com documentos sobre um dado conjunto de classes. A partir da década de 90 essa perspectiva vem sendo mudada; paradigmas de Aprendizado de Máquina na construção de métodos preditivos para automatizar categorização de textos têm sido utilizados (Sebastiani 2002). Métodos preditivos utilizam exemplos de documentos com tópicos conhecidos e tentam encontrar padrões para generalizar regras que podem ser aplicadas à novos documentos. Text Mining assume um caráter preditivo quando são necessários padrões para determinar futuras buscas dentro de grandes massas de textos (Weiss \& Indurkhya 1998).

Segundo Aas \& Eikvil (1999) a maioria das pesquisas em categorização de textos tem sido voltada para um problema binário: o documento é classificado como relevante ou não relevante com respeito a um tópico pré-definido. Entretanto, há muitas fontes de textos tais com News na internet, e-mail e bibliotecas digitais, que são compostas de diferentes tópicos, os quais envolvem um problema de categorização multi-classes. Apesar de muitos documentos pertencerem a mais de uma classe, até hoje foi dada muito pouca atenção a esse tipo de problema.

Algumas pesquisas, em destaque, envolvendo categorização são: 
- Modelo baseado em regras de indução (Apté, Damerau, \& Weiss 1994a).

- Aprendizado automatizado de regras de decisão (Apté, Damerau, \& Weiss 1994a).

- Modelo combinado estatístico e relacional (Craven \& Slattery 1998).

- Algumas técnicas têm sido combinadas. Por exemplo, clustering de documentos para posterior classificação (McCallum \& Baker 1998).

- Comparação de métodos para categorização: Support Vector. Machines, Vizinho Mais Próximo, Rede Neural, Naive Bayes (Yang \& Liu 1999).

\subsubsection{Clustering}

Clustering é uma tarefa de aprendizado não supervisionado, que procura identificar um conjunto finito de clusters a partir dos dados. Ela é útil por facilitar a exploração de um conjunto de dados não muito bem conhecido, dando uma visão geral do seu conteúdo, facilitando o processo de manuseios no sentido de encontrar informações relacionadas.

Por se tratar de uma tarefa de aprendizado não supervisionado, não há uma divisão em conjuntos de treinamento e teste. Além disso, técnicas probabilísticas têm sido usadas para avaliar relações estatísticas entre termos e coleçōes de textos (Willett 1988).

Clustering é diferente da classificação, pois a primeira visa criar os clusters através da organização dos elementos, enquanto que a segunda procura alocar elementos em classes já pré-definidas.

Os elementos a serem agrupados podem ser documentos (textuais ou multimídia), consultas, termos ou expressōes da linguagem, desde que sejam representados da mesma maneira. A separação dos elementos é feita com base numa avaliação de similaridade entre os elementos, procurando colocar os elementos mais similares no mesmo grupo. Para tanto, devem ser escolhidas as características que representarão os objetos.

Segundo Nilsson (1996), clustering pode ser realizado utilizando duas técnicas:

1. O algoritmo aprende por meio de cálculo de probabilidade envolvendo independência condicional e regra de Bayes. Um algoritmo que utiliza essa técnica é o AutoClass. 
2. O algoritmo aprende usando alguma medida de similaridade. Alguns algoritmos que utilizam essa técnica são $K$-Means e Vizinho mais Próximo.

Em Text Mining clustering pode ser usado, por exemplo, para (Tkach 1998):

- Providenciar uma visão geral do conteúdo de uma grande coleção de documentos.

- Identificar similaridades escondidas.

- Facilitar o processo de busca para encontrar informaçōes relacionadas e/ou similares.

Além disso, vale ressaltar que clustering pode ser usado para especificação ou identificação do atributo-meta para ser usado posteriormente em categorizarão de textos $\dot{\jmath}$ (McCallum \& Baker 1998).

\subsection{Considerações Finais}

Com o crescimento da Internet, o acesso a textos sobre os mais diferentes assuntos, oriundos de diversas fontes, criou uma sobrecarga de informações que não são facilmente assimiladas. Assim, algumas técnicas já bastante conhecidas de Data Mining foram adaptadas para serem aplicadas em textos com a mesma eficácia que em outros tipos de dados. A extração de conhecimento em textos é um pouco mais complexa que em base de dados convencionais, pois textos não possuem um formato definido.

Neste capítulo foi apresentada uma visão geral dos elementos que fazem parte do processo Text Mining. Apresentou-se as principais etapas do processo de Text Mining e algumas áreas de apoio para que essas etapas possam ser desenvolvidas. Também foram apresentadas algumas técnicas utilizadas em Text Mining.

No próximo capítulo são apresentadas algumas técnicas, algoritmos e ferramentas utilizadas na análise de textos. 


\section{Capítulo 3}

\section{Algoritmos e Ferramentas para Categorização de Textos}

\subsection{Considerações Iniciais}

Com o aumento do número de pesquisadores interessados na área de Text Mining, tem havido um aumento na exploração e na aplicação de técnicas de aprendizado voltadas para essa área.

Independentemente da abordagem de aprendizado adotada, pode-se resumir o processo de categorização de textos em 6 etapas principais. Na Figura 3.1 é mostrado um esquema geral do processo de categorização de textos utilizando algoritmos de aprendizado, cujas etapas podem ser definidas como:

- Coleta de Documentos: primeira etapa do processo e tem como função recuperar os documentos que possam ser relevantes. Os documentos coletados podem estar em diferentes formatos, havendo assim, a necessidade de padronizá-los em um formato único.

- Identificação de features: formação de um dicionário que contenha as features (ou atributos) que serão utilizados para representar os documentos individuais dentro de uma coleção.

- Representação de Documentos: consiste em mapear cada documento em um 
exemplo de treinamento e associá-lo a um rótulo que identifique sua categoria, tal qual uma tabela atributo/valor que represente o conteúdo de uma coleção de documentos, através da sua freqüência ou da presença/ausência da palavra (booleana).

- Seleção de Features: uma possível extração/redução de features, a fim de diminuir o número de features para posterior utilização dos algoritmos de aprendizado.

- Escolha do Algoritmo de Aprendizado: escolha dos algoritmos, principalmente aqueles utilizados em Aprendizado de Máquina.

- Classificação dos documentos: etapa de avaliação para a escolha da melhor solução, baseada na minimização do erro ou de outros parâmetros que foram utilizados para comparação.

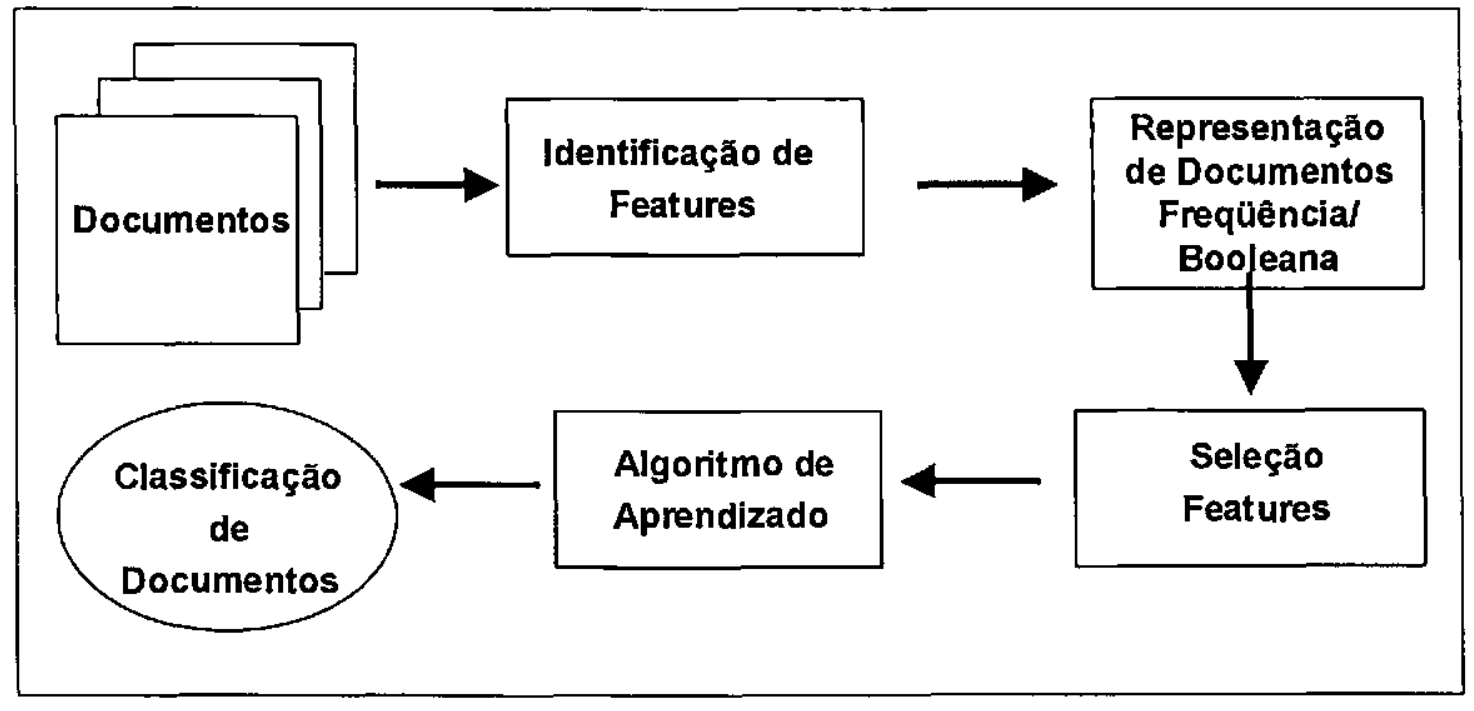

Figura 3.1: Esquema do processo de categorização de textos utilizando algoritmos de aprendizado de máquina

Na Tabela 3.1, adaptada de (Mladenić 1998) tem-se um resumo das principais técnicas utilizadas em Text Mining com seus principais pesquisadores. Nessa tabela são relacionados os trabalhos de alguns autores com a forma usada para representar os documentos, bem como os métodos de aprendizado utilizados. A representação de documentos no formato bag of words é uma representação na qual cada documento é descrito por um 
vetor de features. Há uma feature para cada palavra importante do documento, como mostrado no exemplo da Figura 3.2.

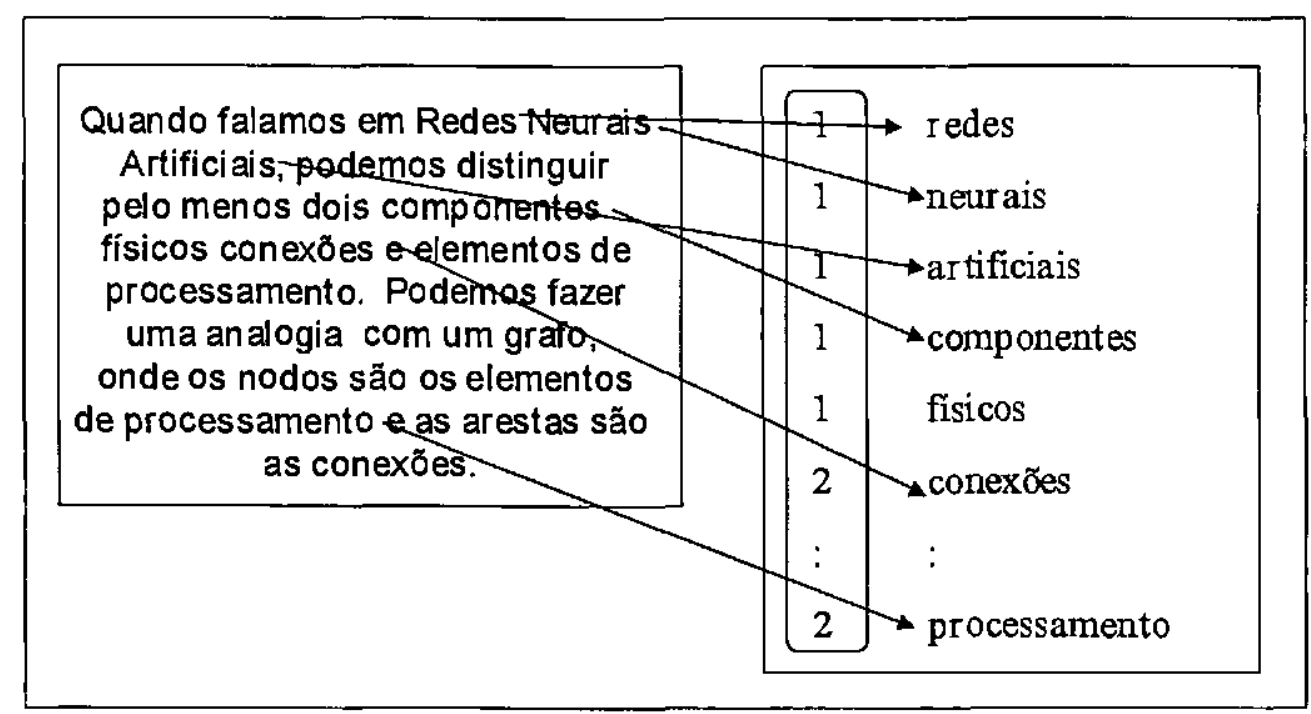

Figura 3.2: Representação do documento no formato bag of word

Neste capítulo são descritos algumas tarefas e alguns dos algoritmos mais utilizados para o tratamento de documentos textuais, bem como algumas ferramentas disponíveis no $\mathrm{LABIC}^{1}$ que foram utilizadas neste trabalho.

\subsection{Alguns Algoritmos Utilizados para Text Mining}

Nos últimos anos tem aumentado o interesse em aplicar métodos de Aprendizado de Máquina para uma variedade de problemas em categorização de textos. Além disso, existe hoje uma certa tendência em se combinar mais de um algoritmo para se conseguir melhores resultados, como aprendizado estatístico (Naive Bayes) com aprendizado relacional (Craven \& Slattery 1998) e clustering com categorização (McCallum \& Baker 1998).

Uma variedade de algoritmos de aprendizado tem demonstrado razoável eficiência para categorização e clustering de textos (McCallum, Nigam, \& Lafferty 1999; Sebastiani 2002), sendo os principais:

- Vizinho mais próximo (Yang 1999).

\footnotetext{
${ }^{1}$ Laboratório de Inteligência Computacional do ICMC - USP : http://labic.icmc.sc.usp.br/
} 


\begin{tabular}{|c||c|c|}
\hline \hline $\begin{array}{c}\text { Referência do } \\
\text { Pesquisador }\end{array}$ & $\begin{array}{c}\text { Representação do } \\
\text { Documento }\end{array}$ & $\begin{array}{c}\text { Algoritmo/Métodos de } \\
\text { Aprendizado }\end{array}$ \\
\hline \hline (Apté, Damerau, \& Weiss 1994b) & bag of words (freqüência) & Regras de Decisão \\
\hline (Apté, Damerau, \& Weiss 1994a) & bag of words & Regras de Decisão \\
\hline (Cohen 1995c) & bag of words & Aprendizado Relacional \\
\hline (Cohen 1995b) & bag of words & Regras de Decisão \\
\hline (Joachims 1997) & bag of words (freqüência) & Naive Bayes \\
\hline (Lewis \& Gale 1994) & bag of words & Naive Bayes \\
\hline (Pazzani \& Billsus 1997) & bag of words & Naive Bayes, \\
& & Aizinho Mais Próximo, \\
& bag of words & Vizinho Mais Próximo de Decisão \\
\hline (Yang \& Pedersen 1997) & bag of words & Vizinho Mais Próximo, \\
& & Support Vector Machines, \\
(Yang \& Liu 1999) & Naive Bayes \\
\hline \hline
\end{tabular}

Tabela 3.1: Representação de documentos, algoritmo de aprendizado usados em trabalhos relacionados

- Naive Bayes (Lewis 1998; McCallum \& Baker 1998).

- Algoritmos que geram Árvores de Decisão (Apté, Damerau, \& Weiss 1998; Apté \& Weiss 1997).

- Algoritmos que geram Regras de Decisão (Cohen \& Singer 1996; Craven \& Slattery 1998).

- Redes Neurais Artificiais (Lagus 2000).

- AutoClass (Cheeseman, Kelly, Self, Stutz, Taylor, \& Freeman 1990).

- K-Means (Padilha 1999; Mitchell 1997).

- Support Vector Machines (Joachims 1998; Dumais, Platt, Heckerman, \& Sahami 1998; Aas \& Eikvil 1999).

\subsubsection{Vizinho Mais Próximo}

Segundo Ling \& Wang (1997) Vizinho Mais Próximos é um dos mais estudados e popular algoritmos de aprendizado. Originou-se do campo de reconhecimento de padrões e 
tem sido bastante utilizado em tarefas de classificação e clustering (Cover \& Hart 1967; McAuliffe \& Afifi 1984).

Esse algoritmo determina a classificação de exemplos de teste de acordo com a sua similaridade (proximidade) com os exemplos de treinamento. Essa classificação pode ser dividida da seguinte forma:

- Armazenar todos os exemplos de treinamento em um mesmo espaço;

- Definir um número $k$ de exemplos mais próximos;

- Utilizar uma função para calcular a similaridade de $x$ (exemplo de teste) com o conjunto $y$ de exemplos de treinamento.

O algoritmo seleciona os $k$ exemplos do conjunto de treinamento que se encontram mais próximos do exemplo a ser classificado, sendo o valor de $k$ previamente escolhido. A categoria atribuída ao exemplo não classificado será aquela da maior parte dos exemplos dentre os $k$-ésimos selecionados. Portanto, o algoritmo do Vizinho Mais Próximo nunca forma uma hipótese geral para todos os exemplos, determinando apenas a classe de cada exemplo de teste fornecido.

Uma medida bastante utilizada para calcular similaridade é a distância Euclidiana, que descreve o menor caminho entre dois pontos $i$-dimensionais:

$$
d(x, y)=\sqrt{\sum_{i}\left(x_{i}-y_{i}\right)^{2}}
$$

Nessa distância, $x$ corresponde ao exemplo de teste, $y$ corresponde ao exemplo de treinamento armazenado, $x_{i}$ e $y_{i}$ são os valores normalizados do $i$-ésimo atributo. A função de similaridade descrita na Equação 3.1 trata todos os atributos igualmente, o que para muitas aplicações não é interessante.

Muitas vezes, um determinado atributo pode ser completamente irrelevante para a classificação, não devendo ser incluído na função de similaridade. Uma função de similaridade mais eficiente para esses casos é mostrada na Equação 3.2:

$$
d(x, y)=\sqrt{\sum_{i} w_{i}^{2} \times\left(x_{i}-y_{i}\right)}
$$

na qual o termo $w_{i}$ é uma constante determinada pelo usuário que identifica a importância (peso) do i-ésimo atributo na função de similaridade (Ling \& Wang 1997). Fixar os 
pesos apropriadamente na função de similaridade melhora a generalização do algoritmo do Vizinho Mais Próximo, especialmente em domínios com atributos menos relevantes para a classificação.

Intuitivamente, aos atributos mais importantes devem ser associados pesos maiores do que aos atributos menos importantes. Estudos mostram que os pesos dos atributos podem ser determinados por um algoritmo de aprendizado, tal como: ID3 ou C4.5 (Ling \& Wang 1997).

\subsubsection{Naive Bayes}

O algoritmo Naive Bayes é baseado na abordagem Bayesiana, projetando um classificador com base nas probabilidades incondicionais do atributo-meta a partir do conjunto de treinamento. A entrada desse algoritmo consiste de um conjunto de dados no formato atributo/valor (Mitchell 1997).

O classificador Naive Bayes se baseia na suposição simplificada de que os vários atributos dos exemplos de entrada são condicionalmente independentes, dado o valor final da função de saída. Assim, esse classificador considera que a probabilidade de ocorrência de uma conjunção de atributos em um dado exemplo é igual ao produto das probabilidades de ocorrência de cada atributo isoladamente. Assumir a independência é claramente incorreto e produz uma probabilidade incorreta dos membros. Mesmo sabendo que ao assumir essa independência o Naive Bayes produz uma estimativa de probabilidade imprecisa, é ainda possível classificar exemplos de teste usando Naive Bayes com uma alta precisão (Craven, McCallum, DiPasquuo, Mitchell, Freitag, \& Nigam 1998).

Naive Bayes classifica um novo exemplo associando a classe mais provável, $v_{M A P}$, a partir dos valores de seus atributos $\left(a_{1}, a_{2}, \ldots, a_{n}\right)$ pela seguinte equação:

$$
v_{M A P}=\max _{v_{j} \in V} P\left(v_{j} \mid a_{1}, a_{2}, \ldots, a_{n}\right)
$$

Essa equação pode ser reescrita utilizando a regra de Bayes:

$$
v_{M A P}=\max _{v_{j} \in V} \frac{P\left(a_{1}, a_{2}, \ldots, a_{n} \mid v_{j}\right) P\left(v_{j}\right)}{P\left(a_{1}, a_{2}, \ldots, a_{n}\right)}
$$


Para determinar a probabilidade de uma classe $P\left(v_{j}\right)$ em um conjunto de dados, basta contar a freqüênicia que cada $v_{j}$ ocorre no conjunto de treinamento. Entretanto, a determinação dos termos $P\left(a_{1}, a_{2}, \ldots, a_{n} \mid v_{j}\right)$ dessa maneira é difícil, principalmente em conjuntos de dados muito grande. O problema é que o número de termos é igual ao número de atributos possível vezes o número de classes possíveis. Dessa forma, é necessário observar várias vezes cada exemplo para obter uma estimativa confiável.

Em um classificador Naive Bayes, a predição da classe de um exemplo é o produtório das probabilidades dos atributos individuais: $P\left(a_{1}, a_{2}, \ldots, a_{n} \mid v_{j}\right)=\prod_{i} P\left(a_{i} \mid v_{j}\right)$. Substituindo essa expressão na Equação 3.4, tem-se a abordagem usada pelo classificador Naive Bayes:

$$
v_{N B}=\max _{v_{j} \in V} P\left(v_{j}\right) \prod_{i} P\left(a_{i} \mid v_{j}\right)
$$

na qual $v_{N B}$ denota o valor da classe do classificador Naive Bayes.

É importante notar que no classificador o número de termos distintos $P\left(a_{i} \mid v_{j}\right)$, que deve ser estimado dos dados de treinamento, é o número de atributos distintos vezes o número de classes distintas (Mitchell 1997).

\subsubsection{Algoritmos que geram Árvores de Decisão}

Uma Árvore de Decisão é uma estrutura de dados recursiva definida como: um nó folha que indica o rótulo de uma classe ou um nó de decisão que contém um teste baseado nos valores de um subconjunto dos atributos de entrada. O nó inicial é chamado de nó raiz. Cada nó representa um teste ou uma decisāo, cujo resultado cria um ramo e uma sub-árvore. Cada sub-árvore tem a mesma estrutura da árvore (Baranauskas \& Monard 2000). No caso de uma árvore binária, os testes podem ser avaliados como verdadeiro ou falso. Um sistema que gera Árvores de Decisão simplesmente particiona uma tabela em pequenas tabelas selecionando subconjuntos baseados em valores para um dado atributo. Existem diferentes algoritmos para indução de Árvores de Decisão, como C4.5 (Quinlan 1993), CART (Breiman, Friedman, Olshen, \& Stone 1984), ID3 (Breiman, Friedman, OIshen, \& Stone 1984), C5. $0^{2}$ (Rulequest-Research 1999).

\footnotetext{
${ }^{2} C 5.0$ é a versão para UNIX e similares e See5 é a versão para Windows95/NT
} 
Para que uma Árvore de Decisão classifique um caso, primeiramente, o nó raiz é testado. Dependendo do resultado do teste, o processo seguirá a ramificação direita ou esquerda em direção a um outro nó. $O$ processo se repete até que um nó terminal (também chamado de nó folha) seja encontrado, rotulando um valor padrão que classifique o exemplo. Esse valor pode ser categórico (Classificação) ou real (Regressão), dependendo dos valores do atributo-meta.

A complexidade da solução representada pela Árvore de Decisão depende do número de nós-folha. Árvores complexas são menos intuitivas para seres humanos do que árvores mais simples, sendo que a complexidade da árvore pode ser melhorada utilizando-se técnicas de poda.

Algoritmos de aprendizado como C4.5 (Quinlan 1993), OC1 (Murthy, Kasif, \& Salzberg 1994) e C5.0 (Rulequest-Research 1999) são os mais utilizados para gerar Árvores de Decisão (Baranauskas \& Monard 2000).

\subsubsection{Algoritmos que geram Regras de Decisão}

Regras de Decisão (Quinlan 1993) são relações lógicas que geralmente utilizam relações condicionais e associativas. Uma relação condicional é uma declaração da forma:

$\mathrm{SE}<$ condição $>\mathrm{ENTÃO}<$ classe $=X>$.

As Regras de Decisão são capazes de manipular tanto dados numéricos quanto categóricos de forma uniforme. As Regras de Decisão são potencialmente mais poderosas que Árvores de Decisão. Embora todas as Árvores de Decisão possam ser expressas na forma de Regras de Decisão, a diminuição nas restrições de exclusão mútua pode potencialmente levar as regras a cobrir as classes de forma mais eficiente e compacta (Batista 1997). Além disso, as Regras de Decisão conseguem manipular valores incompletos devido a sobreposição de regras disjuntas.

A complexidade de uma Regra de Decisão é medida pelo número total de termos que ela consegue generalizar (Quinlan 1993; Weiss \& Indurkhya 1998). Normalmente, quando a Regra de Decisão é aplicada em bases multidimensionais, a complexidade do entendimento é comprometida. Para diminuir a complexidade é necessário utilizar técnicas de poda para que o conjunto de termos de uma Regra de Decisão seja reduzido. 
Algoritmos de aprendizado como CN2 (Clark \& Boswell 1991; Clark \& Niblett 1989), C4.5 rules (Quinlan 1987), Ripper (Cohen 1995a) e C5.O (Rulequest-Research 1999) são os mais utilizados para gerar Regras de Decisão (Baranauskas \& Monard 2000).

\subsubsection{Redes Neurais Artificiais}

O paradigma conexionista, também conhecido como Redes Neurais Artificiais (RNAs), representa uma forma de computação não algorítmica, cujo funcionamento é inspirado na estrutura e funcionamento do cérebro humano. As RNAs podem ser definidas como sistemas paralelos distribuídos compostos de unidades de processamento simples, altamente interconectadas, que computam determinadas funções matemáticas. Tais unidades são dispostas em uma ou mais camadas e interligadas por um grande número de conexões, geralmente unidirecionais. Na maioria dos modelos, essas conexões possuem pesos, os quais armazenam o conhecimento representado no modelo e servem para ponderar a entrada recebida por cada neurônio da rede (Braga, Carvalho, \& Ludermir 2000). Enquanto um sistema convencional opera sobre algoritmos, regras e processamento exato, uma RNA explora diversas hipóteses que competem entre si simultaneamente.

A solução de problemas através de RNAs é bastante atrativa, já que a forma como eles são representados internamente pela rede e o paralelismo natural inerente à arquitetura das RNAs criam a possibilidade de um desempenho superior àquele obtido por modelos convencionais. Em RNAs, o procedimento usual na solução de problemas passa inicialmente por uma fase de aprendizagem, na qual um conjunto de padrões ou exemplos é apresentado à rede, que extrai automaticamente as características necessárias para representar a informação fornecida. Essas características são utilizadas posteriormente para gerar as respostas para novos padrões.

Resultados sobre o uso de redes neurais em Text Mining e categorização de textos podem ser encontrados em (Lagus 2000).

\subsubsection{AutoClass}

AutoClass é um algoritmo de clustering não supervisionado, baseado na abordagem Bayesiana, que procura uma classificação de probabilidade máxima. Os clusters são descritos de acor- 
do com uma probabilidade, de forma que um objeto pode ser membro parcial de diferentes clusters e as definições dos clusters podem se sobrepor. AutoClass gera relatórios sobre os clusters encontrados ao final de sua busca.

Vale lembrar que nessa abordagem, os clusters são descritos a partir da distribuição probabilística sobre os atributos dos exemplos, especificada por uma função modelo e por seus parâmetros (Cheeseman, Kelly, Self, Stutz, Taylor, \& Freeman 1990).

Algumas características do AutoClass são:

- determina o número de classes automaticamente;

- pode usar dados discretos e reais;

- pode lidar com dados incompletos ou valores ausentes;

- o tempo de processamento é linear na quantidade de dados;

- permite correlação entre atributos dentro de uma classe; e

- gera relatórios descrevendo as classes encontradas.

A entrada desse algoritmo consiste de um conjunto de dados, no formato atributo/valor. O AutoClass encontra um conjunto de clusters que seja altamente provável com os dados e modelos especificados. A saída desse algoritmo é a descrição probabilística dos clusters identificados e dos exemplos pertencentes a esses clusters.

No algoritmo AutoClass, a determinação do número de clusters para uma clustering é opcional, ou seja, o usuário tem a opção de fornecer o número de clusters que deseja numa determinada clustering. Caso o usuário não determine esse número, o algoritmo tenta encontrar os clusters automaticamente para o melhor clustering. $\mathrm{O}$ uso do conhecimento de fundo pode auxiliar na definição do número de clusters. Decidir quando deve ser suspensa a formação de clusters é uma questão fundamental desse algoritmo.

Nesse algoritmo, todos os atributos do conjunto de dados são potencialmente relevantes, representando uma vantagem sobre clustering humana, que permite a manipulação de poucos atributos. O AutoClass utiliza todos os atributos simultaneamente, permitindo uma consideração uniforme de todos os dados. 
No AutoClass, os relacionamentos entre os clusters são descritos por meio de probabilidades. Dessa forma, cada exemplo tem uma determinada probabilidade de pertencer a um ou alguns dos possiveis clusters. Esse relacionamento probabilístico entre clusters deve somar um para cada exemplo.

Quando um exemplo possui uma probabilidade maior que 0,99 em um cluster, é provável que os clusters sejam bem separados e também bem definidos. Se existem alguns exemplos que possuam uma probabilidade $0,5 \mathrm{em}$ algum cluster, isso significa que alguns clusters estão sobrepostos. Nesse caso, a combinação de clusters nos conjuntos de dados é usualmente mais difícil do que considerar cada cluster separadamente.

Embora o AutoClass seja designado para a tarefa de clustering, o mesmo também permite classificação supervisionada. Isso acontece após o treinamento, quando o usuário deseja confirmar se alguns exemplos estão em determinadas classes (Cheeseman, Kelly, Self, Stutz, Taylor, \& Freeman 1990).

\subsubsection{K-Means}

O K-Means é um algoritmo de clustering que tem como finalidade agrupar vários exemplos num determinado número de clusters. Os agrupamentos dos exemplos são realizados utilizando alguma medida de similaridade, tais como: Euclidiana e Manhattan. Esse algoritmo está disponível no software Mineset ${ }^{T M}$, descrito na Seção 3.3.2.

No método K-Means, a distância de um ou mais pontos centrais para todos elementos serve como medida para criar os agrupamentos. A utilização de uma média faz com que esse método seja mais indicado para problemas onde as variáveis são numéricas.

O K-Means tem como finalidade encontrar um número de clusters que esteja entre os limites inferior e superior definido. Cada modelo de clustering criado é avaliado pela média de dispersão de cada cluster. Nesse método, o ponto de escolha seleciona o grau desejado de dispersão, medido como a proporção de uma dispersão entre o número mínimo e máximo de clusters.

Os clusters gerados são nomeados com base na derivação dos mesmos durante o processo de divisão. Toda vez que um cluster é dividido, os dois clusters novos são nomeados com base no cluster que foi dividido. 
Um fator importante nos modelos de clustering é que as medidas de dispersão nos clusters não são imediatamente comparáveis quando se determina conjuntos de dados ou números de clusters diferentes. Com o propósito de descobrir modelos de clustering mais compreensíveis, o usuário pode associar pesos (valores numéricos) aos atributos dos conjuntos de dados. Esses pesos definem que os atributos tenham uma influência maior ou menor para a realização de clustering.

Mais detalhes podem ser encontrados em (Padilha 1999; Rezende, Paula, \& Figueiredo 1998).

\subsubsection{Support Vector Machines}

Support Vector Machines (SVM) (Vapnik 1995) englobam uma classe de algoritmos de aprendizado que se baseiam na teoria de aprendizado estatístico, combinando controle de generalização com uma técnica para tratar o problema da alta dimensionalidade. Sua formulação resulta em um problema de otimização quadrática e envolve o princípio de Minimização do Risco Estrutural (Structural Risk Minimization - SRM), em vez do princípio de Minimização do Risco Empírico (Empirical Risk Minimization - ERM) empregado pelas redes neurais convencionais. SRM minimiza o erro de generalização em oposição ao ERM que minimiza o erro nos dados de treinamento, dando ao algoritmo SVM uma maior capacidade de generalização.

Esse tipo de algoritmo foi originalmente desenvolvido para reconhecimento de padrões. Nesse caso, uma fronteira de decisão é representada em termos de um subconjunto dos exemplos de treinamento, tipicamente pequeno, chamado de vetores de suporte (support vectors).

Esse algoritmo será descrito detalhadamente no Capítulo 4, pelo fato de ser um dos algoritmos mais explorados em Text Mining, e também o mais explorado neste trabalho.

\section{Método de Resampling Cross-Validation}

Todos os sistemas de Aprendizado de Máquina e Text Mining devem ser avaliados para determinar a taxa de erro do classificador, normalmente calculada a partir de um conjunto de teste que não possua exemplos em comum com o conjunto de treinamento. 
Tanto o conjunto de treinamento quanto o conjunto de teste devem ser amostras aleatórias do conjunto de dados. Um método que se utiliza desses critérios, sendo que todos os exemplos da amostra são usados para testar e todos os exemplos são usados para treinar o sistema, é o método de resampling cross-validation, também conhecido com $k$ fold cross-validation, no qual $k$ representa o número de partiçōes geradas aleatoriamente no conjunto de dados para treinar e testar o sistema.

A amostra de exemplos é dividida em $k$ partições mutuamente exclusivas. A cada iteração, todas as $k$-1 partições são utilizadas para treinar o sistema e uma partição diferente é usada para testar o sistema de aprendizado. A taxa de erro é a média das taxas de erro calculadas para as diversas partições, bem como outras medidas utilizadas.

\subsection{Algumas Ferramentas Disponíveis}

Diversas ferramentas foram utilizadas na realização deste trabalho. Dentre elas destacamse:

- $\mathcal{M L C}++$.

- MineSet ${ }^{T M}$.

- $S V M^{\text {light }}$.

- Weka.

\subsection{1 $\mathcal{M L C}++$}

A $\mathcal{M L C}++$ é uma biblioteca de classes e ferramentas para Aprendizado de Máquina, implementada na linguagem $C++$, que foi projetada para ajudar na escolha de algoritmos para realizar tarefas específicas e/ou no desenvolvimento de novos algoritmos (Kohavi 1994; Kohavi, Sommerfield, \& Dougherty 1996).

O projeto da $\mathcal{M L C}++$ começou em 1993, na Universidade de Stanford, estando sob a responsabilidade da Silicon Graphics desde 1995. A $\mathcal{M L C}++$ é um software de domínio público (inclusive os códigos fontes).

A biblioteca $\mathcal{M L C}++$ tem um papel duplo: é utilizada para desenvolver algoritmos, e também utilizada por usuários finais. A biblioteca $\mathcal{M L C}++$ contém mais de 30 algoritmos 
de Aprendizado de Máquina implementados, além de fornecer utilitários e ferramentas que podem ser usadas separadamente.

A $\mathcal{M L C}++$ baseia-se no fato de que não existe um único algoritmo de Aprendizado de Máquina que forneça ótimos resultados de precisão para todas as tarefas. Portanto, é aconselhável testar vários algoritmos com o mesmo conjunto de dados, para poder comparar e determinar qual algoritmo apresenta melhor desempenho em cada caso.

Uma descrição completa sobre a biblioteca $\mathcal{M L C}++$ e a utilização de alguns algoritmos de Aprendizado de Máquina pode ser encontrada em (Felix, Rezende, Doi, de Paula, \& Romanato 1998).

\subsubsection{Mineset ${ }^{T M}$}

O MineSet ${ }^{T M}$ é um software da Silicon Graphics que possui um conjunto de ferramentas com a finalidade de auxiliar na extração de conhecimento de dados. É uma ferramenta que utiliza a biblioteca de algoritmos de Aprendizado de Máquina $\mathcal{M L C}++$.

O MineSet ${ }^{T M}$ permite a geração e análise de regras de associação e de clusters, classificação e indução de árvores de decisão, regressão, entre outras. Existem ainda outras funções adicionais no $M i n e S e t^{T M}$, tais como: discretização, seleção, criação ou eliminação de atributos, filtragem de exemplos no conjunto de dados e mudança de tipos de dados. Todas essas funcionalidades são apoiadas por ferramentas de visualização (Oliveira \& Rezende 1998).

O MineSet ${ }^{T M}$ é constituído por três componentes básicos:

1. Módulo de Controle Centralizado: consiste de uma interface gráfica chamada Tool Manager e de um processo denominado Data Mover, que permite, entre outras coisas, a interface direta com bases de dados Oracle, Sybase, Informix, bem como arquivos no formato ASCII.

2. Módulo de Ferramentas para Data Mining: consiste de cinco utilitários: gerador de regras de associação, ferramentas de classificação (indutor de árvores de decisão, indutor de árvore de opção e indutor e classificador de evidência), ferramentas para clustering, ferramentas de regressão e importância de colunas. 
3. Ferramentas de Visualização: consiste dos módulos de visualização: visualizador de árvore de decisão, visualizador de mapas, visualizador de regras, visualizador de evidências da classe dentro de cada atributo, visualizador da base ou conjunto de dados, visualizador de estatísticas geradas pelo MineSet $^{T M}$, visualizador difuso bidimensional e tridimensional.

\subsubsection{SVM $M^{\text {light }}$}

Uma das ferramentas mais utilizadas neste trabalho foi o $S V M^{\text {light }}$. Trata-se de uma implementação de SVM para o problema de reconhecimento de padrões para classificação. $S V M^{\text {light }}$ foi desenvolvida Joachims (1998) e possui as seguintes propriedades:

- Trabalha bem com espaço de entrada hiper-dimensional, devido à proteção de overfitting por meio do controle da Dimensão VC ${ }^{3}$. Trata satisfatoriamente de exemplos com muitos atributos, o que invariavelmente ocorre, quando da notação vetorial de documentos.

- Aceita vetores esparsos, que é uma propriedade comum aos vetores de documentos.

- A forma mais simples de indução de SVM's se dá através da obtenção de hiperplanos separadores lineares, ao passo que a maioria dos problemas de categorização de textos é linearmente separável.

O SVM $M^{\text {light }}$ possui dois programas: o sum_learn e sum_classifier.

O sum_learn é o programa responsável pela obtenção do modelo de classificação.

Ele utiliza um conjunto de exemplos de treinamento e desenvolve um modelo de acordo com diversas opções. Sua sintaxe é:

$>$ svm_learn [options] <example_file><model_file>

onde: example_fle indica o arquivo que contém os exemplos de treinamento na forma vetorial, model_file indica o nome do arquivo de saída contendo o modelo para classificação e os argumentos opcionais de options indicam várias opções de configuração do algoritmo. As principais sāo:

\footnotetext{
${ }^{3}$ Dimensão VC será detalhada no Capítulo 4
} 
- -c float: indica a relação entre o erro no conjunto de treinamento e a margem, default: 1000 .

- -j float: fator de custo que indica em quantas vezes o peso em erros de exemplos positivos é maior que o peso de erros em exemplos negativos, default: 1.

- -b [0,1]: restrição $x . w+b>0$ ou $x . w>0$, para o hiperplano (reduz um grau de liberdade do hiperplano separador, facilitando os cálculos dos multiplicadores de Lagrange), default: 1.

- - $[0,1]$ : remove exemplos de treinamento inconsistentes e treina novamente.

- -p [0..1]: fração de exemplos não rotulados que serão classificados como positivos, caso hajam exemplos não rotulados, default:(positivos)/(negativos rotulados)

- -t int: tipo de função kernel.

[0] linear: (default)

[1] polinomial: $(s a * b+c)^{d}$

[2] função: radial basis $\left(-g\|a-b \mid\|^{2}\right)$

[3] sigmóide: $\tanh (s a * b+c)$

- -d int, -g float, -s float, -r float (parâmero c), -u string: parâmetros a serem utilizados em função da escolha da função kernel $(t=1 ; t=2 ; t=3)$, todos definidos pelo usuário.

O sum_classifier é o programa que, utilizando o modelo gerado pelo sum_learn, é aplicado a exemplos de testes, obtendo a sua classificação. A sintaxe desse programa é:

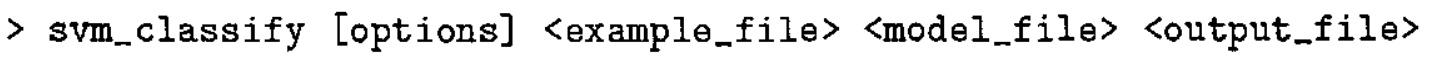

onde: example_fle indica o arquivo que contém os exemplos de teste na forma vetorial, model_file indica o nome do arquivo que contém o modelo para classificação induzido pelo sum_learner e arquivo output_file contém o resultado final do classificador SVM, bem como os argumentos opcionais de options que indicam as opções do programa.

Os arquivos de exemplos utilizados pelo $S V M^{\text {light }}$ apresentam o seguinte formato: 


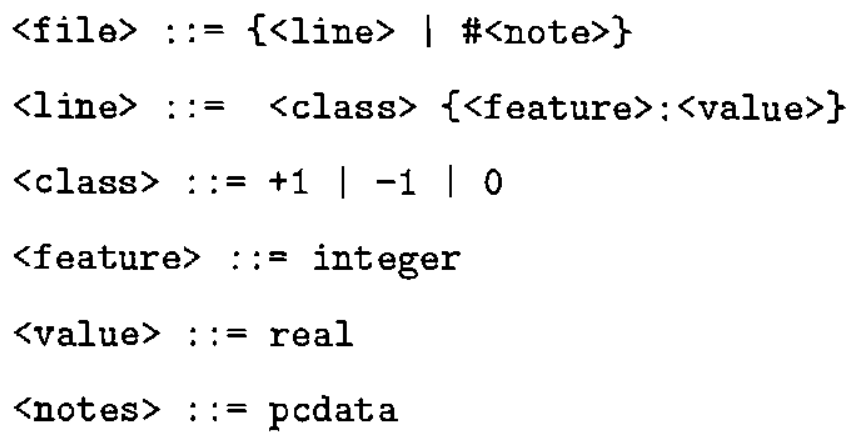

As classes +1 e -1 indicam se o exemplo correspondente é um exemplo positivo ou negativo. Além disso, as linhas iniciadas com \# são consideradas comentário.

Exemplificando: sejam 3 arquivos principais de entrada repres, teste e word para a ferramenta $S V M^{\text {light }}$, conforme ilustrado na Figura 3.3. O arquivo repres é a representação vetorial do exemplo de treinamento, o arquivo teste é a representação vetorial do exemplo de teste e o arquivo word não entra na representação do $S V M^{l i g h t}$, estando mais para exemplificar as features utilizadas.

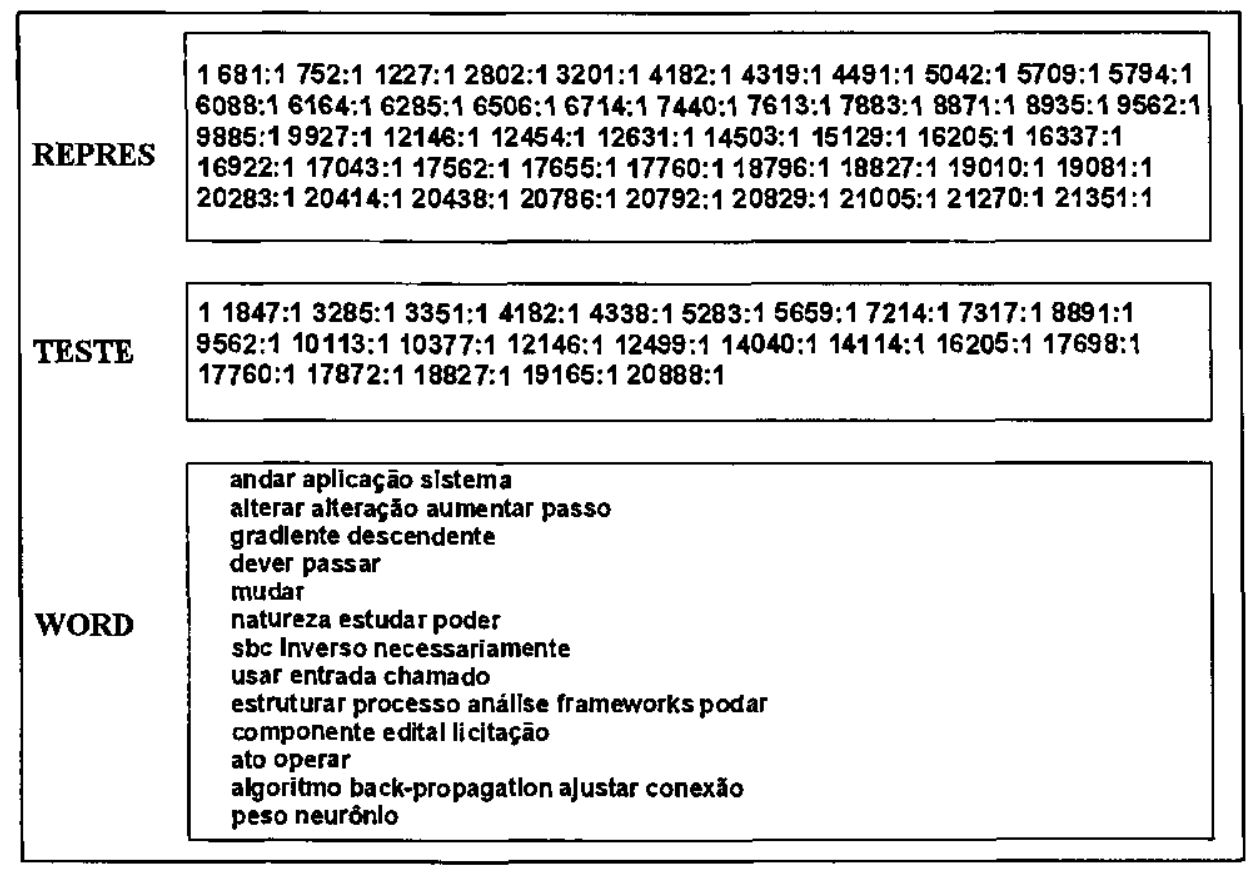

Figura 3.3: Formato dos arquivos para entrada no $S V M^{\text {light }}$ 
Em seguida, com o modelo gerado, é possível extrair o resultado almejado:

lbf $\{$ svm_classify $\}$ teste modelo1 resultado 1

\subsubsection{WEKA}

Weka (Waikato Environment for Knowledge Analysis) ${ }^{4}$ é um ambiente para extração de padrões desenvolvido na linguagem Java. Trata-se de uma coleção de algoritmos de Aprendizado de Máquinas para a resolução de problemas reais de Data Mining. Possui algoritmos para classificação, regressão, clustering e associação.

$\mathrm{O}$ arquivo que contém os exemplos de treinamento para a utilização do Weka possui uma seção de dados, delimitada pela expressão (ou tag) @data, com a representação dos exemplos de forma semelhante à representação do See5/C5.0: cada exemplo corresponde a uma linha, sendo que o valor de cada atributo está listado na ordem especificada pelo esquema de dados (ordem em que os atributos especificados pela expressão @attribute aparecem). A expressão @relation corresponde ao nome do conjunto de dados.

Através dessa ferramenta, tem-se vários algoritmos para classificação: Naive Bayes, Tabela de Decisão, KNN (Vizinho mais Próximo), Árvores de Decisão (C4.5, ID3), Indução de Regras (PART).

A Figura 3.4 apresenta o formato dos dados de entrada para a ferramenta Weka.

\subsection{Considerações Finais}

Neste capítulo foram abordados alguns algoritmos e ferramentas utilizadas neste trabalho. Os dados que são armazenados podem ser recuperados de forma a permitir o uso de técnicas e ferramentas para encontrar modelos e padrões embutidos nesses dados.

Foram descritos alguns dos principais algoritmos utilizados em Text Mining para extração de conhecimento.

No próximo capítulo será descrito em detalhes um dos principais algoritmos para categorização de textos: Support Vector Machines.

\footnotetext{
${ }^{4}$ http://www.cs.waikato.ac.nz/ml/weka
} 


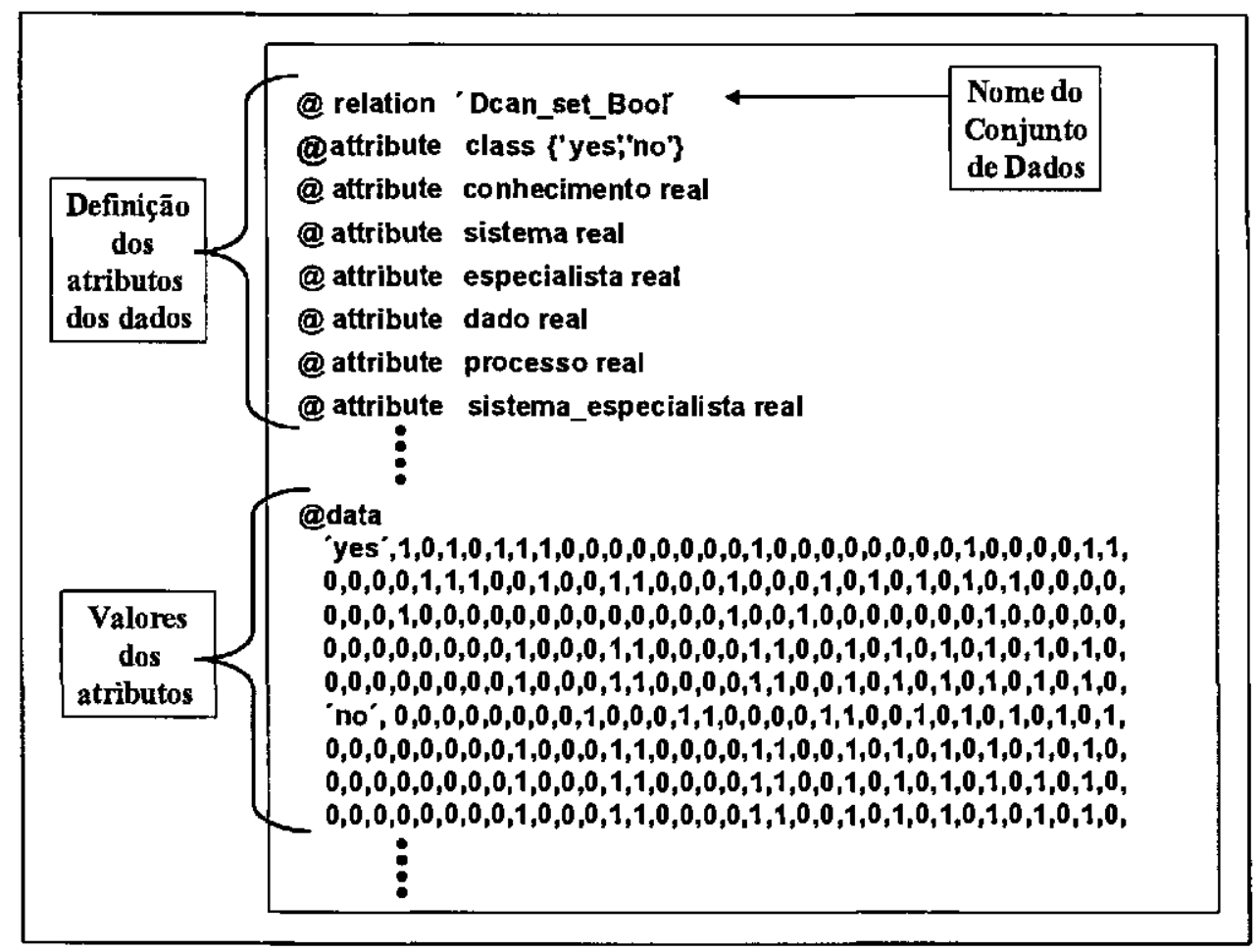

Figura 3.4: Formato dos arquivos para entrada no Weka 


\section{Capítulo 4}

\section{Support Vector Machines}

\subsection{Considerações Iniciais}

Neste capítulo é utilizado o algoritmo de Support Vector Machines (SVM), por ser um dos algoritmos para categorização de textos que mais vêm sendo utilizado e, por conseguinte, tem mostrado ser um dos mais eficientes nessa área (Cooley 1999; Yang \& Liu 1999; Drucker, Wu, \& Vapnik 1999; Joachims 1998; Dumais, Platt, Heckerman, \& Sahami 1998). SVM é um método de Aprendizado de Máquina supervisionado oriundo da Estatística clássica que foi introduzido como uma técnica para reconhecimento de padrões para solução de uma variedade de problemas de aprendizado.

\subsection{Descrição Geral do SVM}

SVM (Vapnik 1995) é um algoritmo de aprendizado e se baseia na teoria de aprendizado estatístico, combinando controle de generalização com uma técnica para tratar o problema da dimensionalidade. Sua formulação resulta em um problema de otimização quadrática e envolve o princípio de Minimização do Risco Estrutural (Structural Risk Minimization - SRM), em vez do princípio de Minimização do Risco Empírico (Empirical Risk Minimization - ERM) empregado pelas redes neurais convencionais. O SRM minimiza o erro de generalização, que é a taxa de erro do algoritmo de aprendizado nos dados de teste, em oposição ao ERM que minimiza o erro nos dados de treinamento. Isso dá ao algoritmo 
SVM uma maior capacidade de generalização. Esse tipo de algoritmo foi originalmente desenvolvido para reconhecimento de padrões. Nesse caso uma fronteira de decisão é representada por um subconjunto dos exemplos de treinamento, tipicamente pequeno, chamado de vetores de suporte (support vectors), extraídos pelo algoritmo.

Em sua forma linear, SVM gera um hiperplano que separa um conjunto de amostras positivas de um conjunto de amostras negativas. Em termos geométricos este método pode ser visto como uma tentativa de busca da melhor superfície $\sigma_{i}$, no conjunto de todas as superfícies $\sigma_{1}, \sigma_{2} \ldots \sigma_{n}$ no espaço r-dimensional que separa os exemplos de treinamento positivos dos negativos (superfície de decisão) (Sebastiani 2002). $\sigma_{i}$ separa os exemplos positivos dos negativos pela mais larga margem possível.

O funcionamento do algoritmo é mais bem compreendido no caso em que os exemplos positivos e negativos são linearmente separáveis, caso em que as superfícies de decisão são $(r-1)$ hiperplanos. O caso de duas dimensões é mostrado na Figura 4.1, em que vários conjuntos de linhas paralelas devem ser escolhidos como superfícies de decisão. Neste caso, é representando os vetores (hiperplanos) que separam classes distintas. SVM tem por objetivo determinar o vetor que maximize a distância entre as classes (máxima margem).

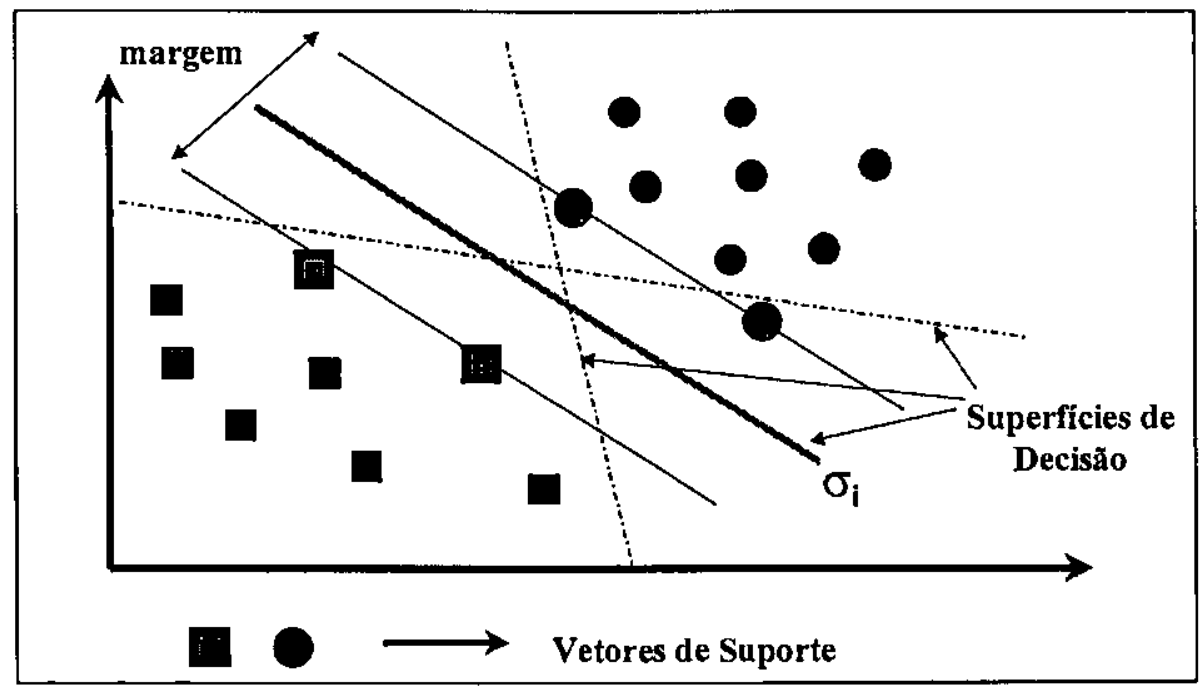

Figura 4.1: Esquema das superfícies de decisão do SVM

Neste caso, dentre as várias superfícies de decisão possíveis, o SVM escolhe aquela 
que maximiza a distância entre as classes (máxima margem). Na Figura 4.2 à direita temse um bom hiperplano com uma margem grande, talvez até o hiperplano ótimo, enquanto à esquerda um hiperplano menos aceitável com uma margem pequena. É intuitivo que com a maior margem há uma melhor eficiência na generalização (Kecman 2001).

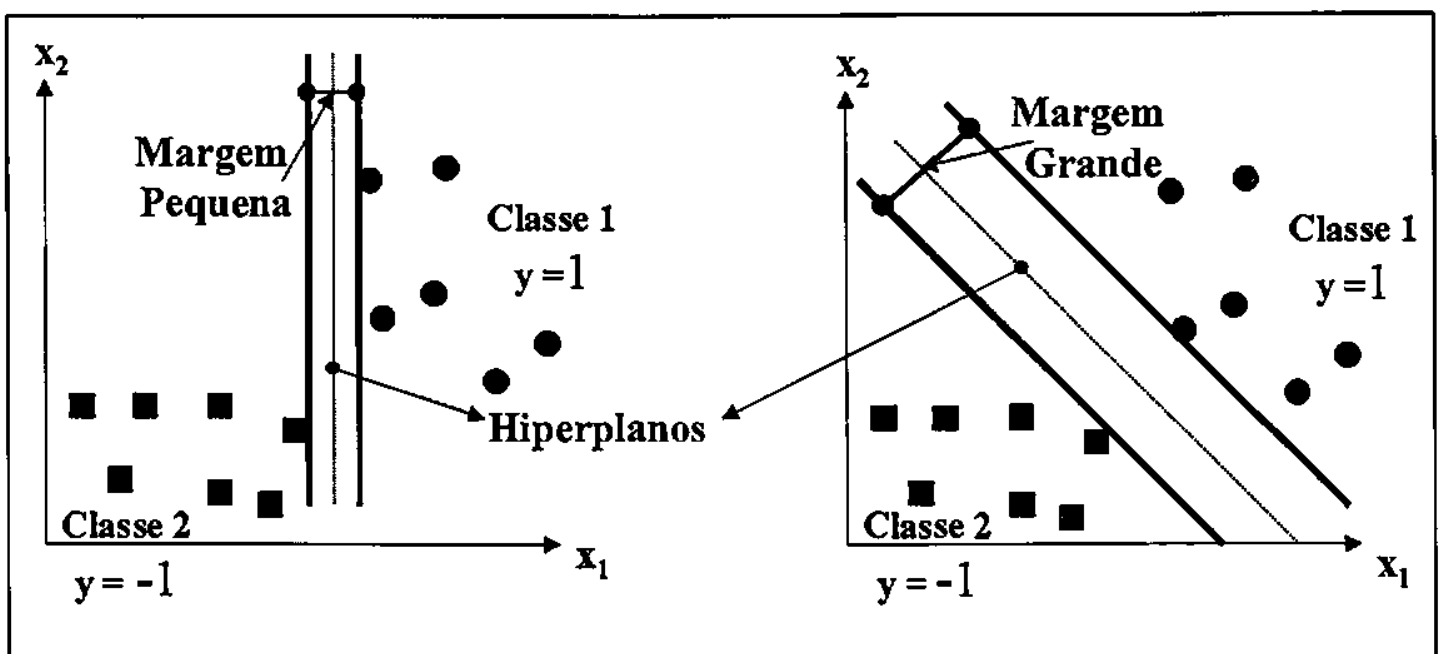

Figura 4.2: Dois dos muitos hiperplanos de separação (Kecman 2001)

SVM possui bom desempenho de generalização numa grande variedade de problemas de classificação, tais como reconhecimento de caracteres manuscritos (Matic, Guyon, Denker, \& Vapnik 1993), reconhecimento de face (Osuna, Freund, \& Girosi 1997; Pontil \& Verri 1998; Roobaert \& Hulle 1999) e mais recentemente, categorização de texto (Joachims 1998; Cooley 1999; Hearst, Scholkopf, Dumais, Osuna, \& Platt 1998).

De acordo com Joachims (1998) SVM tem muitas vantagens para categorização de texto:

- A grande maioria dos problemas de categorização de texto são linearmente separáveis.

- Não há necessidade de redução de atributos, uma vez que SVM suporta alta dimensionalidade, e não apresenta problemas de overfitting.

- Escolhas de padrões (default) tem mostrado excelente eficiência. 
SVM apresenta algumas vantagens em relação à Redes Neurais Artificiais (RNAs): o treinamento de SVM encontra uma solução global, enquanto em RNAs geralmente há mínimos locais; em SVM há minimização do erro de treinamento (risco empírico) como em RNAs e também a minimização do erro de generalização (risco estrutural).

A origem da teoria do SVM é baseada em alguns conceitos de estatística e será visto alguns detalhes que levaram ao surgimento desse algoritmo.

\subsection{Teoria de Aprendizado Estatístico usado em SVM}

Na Figura 4.3 é ilustrado de forma simplificada, o que acontece com o erro em função do tamanho da amostra dos dados. Amostras reais com poucos dados resulta quase sempre em erro elevado, e em termos práticos quase não produz melhoria, quando comparado com dados gerados randomicamente. Para obter boa aproximação ou classificação há necessidade, muitas vezes, de grandes amostras de dados, que normalmente irão produzir menores erros (Kecman 2001).

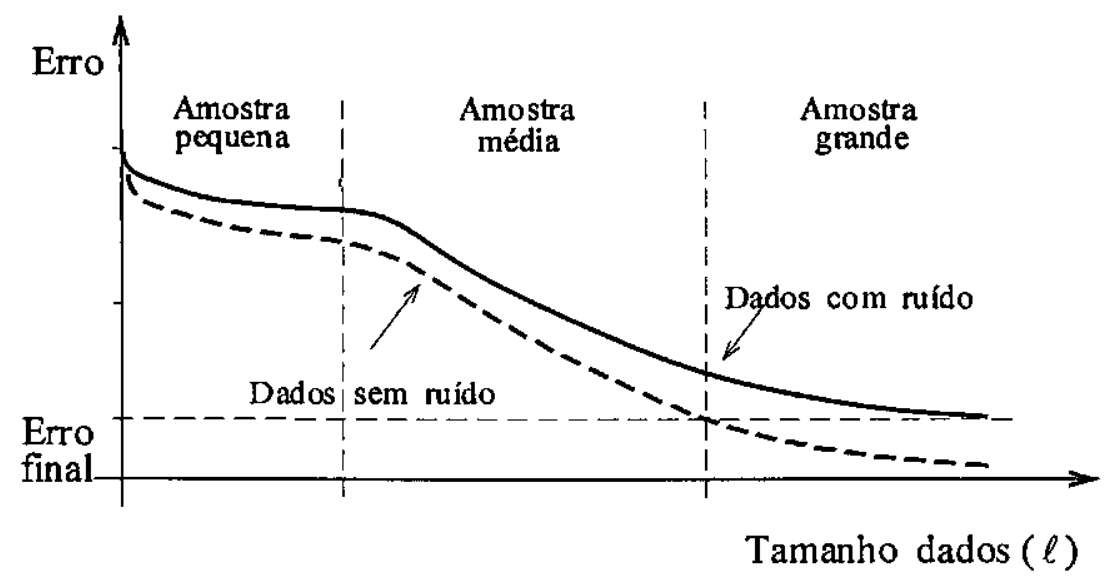

Figura 4.3: Dependência do erro do modelo em relação ao tamanho dos dados de treinamento (Kecman 2001)

Pela sua essência, aprendizado é um processo estocástico. Os conjuntos dos dados de treinamento são formados por dois conjuntos de variáveis: variáveis de entrada $\mathbf{x}_{i}$, a qual é randomicamente, com probabilidade $P\left(\mathbf{x}_{i}\right)$, traçada do conjunto de entrada $\mathbf{X}$, 
e a resposta do sistema $y_{i}$, a qual pertence ao conjunto de saída Y. $y_{i}$ é observado com probabilidade $P\left(y_{i} \mid \mathbf{x}_{i}\right)$. Esta medida (ou observação) resposta $y_{i}$ é denominada $d_{i}$ durante a fase de treinamento. Portanto, $P\left(d_{i} \mid \mathbf{x}_{i}\right)=P\left(y_{i} \mid \mathbf{x}_{i}\right)$. A variável escalar de saída $y$ é usada aqui somente para simplificação. A probabilidade de uma coleção de dados de treinamento $(\mathbf{x}, d)$ é portanto:

$$
P(\mathbf{w}, d)=P(\mathbf{x}) P(y \mid \mathbf{x})
$$

A resposta observada de um sistema é probabilística, e pode ser descrita pela probabilidade condicional $P(y \mid \mathbf{x})$, que exprime para uma mesma entrada $\mathbf{x}$, uma diferente saída $y$ a cada vez. Em outras palavras, não há garantia de resposta y para uma mesma entrada $\mathbf{x}$.

Numa decisão probabilística há 3 componentes básicos em aprendizado de dado: um gerador de entradas randômicos $\mathbf{x}$, um sistema cuja resposta de treinamento $\mathbf{y}$ são usados pelo algoritmo de aprendizado depois de treinado e, um algoritmo de aprendizado que, usando entradas $\mathbf{x}$ e o sistema de resposta $\mathbf{y}$, cria um modelo estimado, como ilustrado pela Figura 4.4.

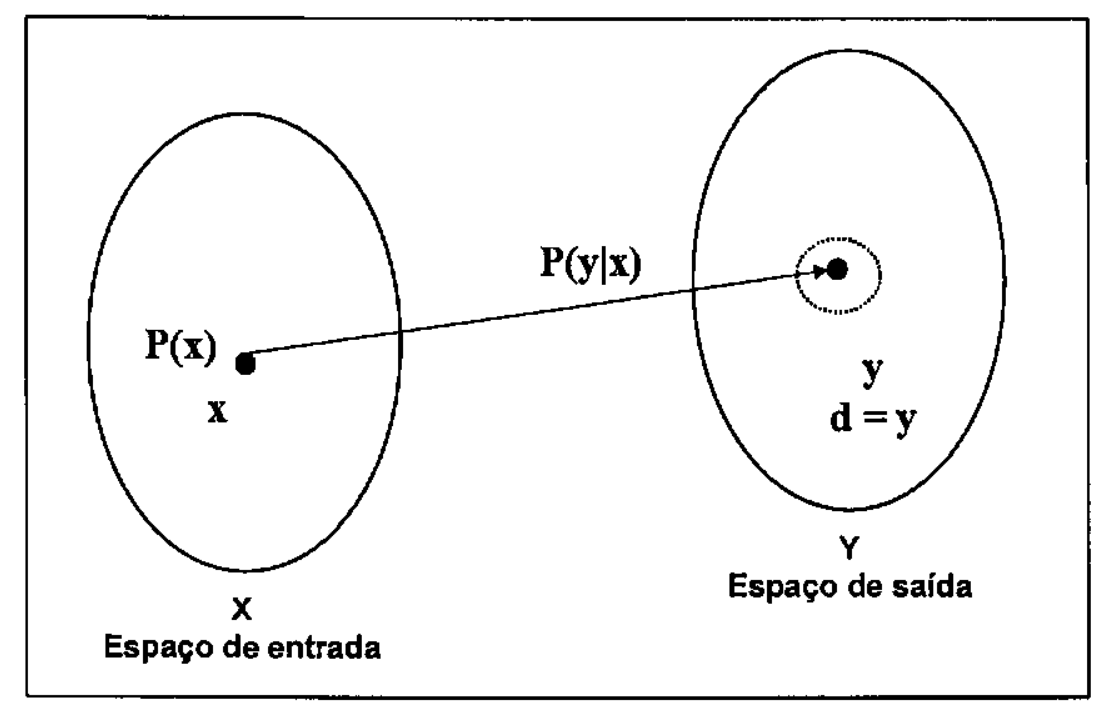

Figura 4.4: Características estocásticas do aprendizado em uma coleção para um conjunto de dados de treinamento (Kecman 2001) 
Durante a fase de treinamento de um algoritmo de aprendizado, pode ser possível encontrar a relação entre $\mathbf{X}$ e $\mathbf{Y}$, usando uma função que separe os dados numa tarefa de classificação. O resultado desse processo de aprendizado é uma função de aproximação $f_{a}(\mathbf{x}, \mathbf{w})$, que na literatura estatística é também conhecida como uma hipótese $f_{a}(\mathbf{x}, \mathbf{w})$.

A escolha da hipótese $f_{a}(\mathbf{x}, \mathbf{w})$ relaciona-se com o espaço de funções $H\left(f_{a} \in H\right)$, e essa é a função que minimiza alguma função risco $\mathbf{R}(\mathbf{w})$. O risco $\mathbf{R}(\mathbf{w})$ é também chamado de perda média esperada e é calculado como:

$$
R(\mathbf{w})=\int L(y, o) d P(\mathbf{x}, y)=\int L\left(y, f_{a}(\mathbf{x}, \mathbf{w})\right) d P(\mathbf{x}, y)
$$

onde a função perda $L\left(y, f_{a}(\mathbf{x}, \mathbf{w})\right)$ é calculada para um conjunto de treinamento $D\left(\mathbf{x}_{\mathbf{i}}, y_{i}\right)$.

A função perda $L(y, o)=L\left(y, f_{a}(\mathbf{x}, \mathbf{w})\right)$ representa alguma função padrão de custo ou função clássica. Dependendo das características do problema, diferentes funções de perda são utilizadas.

Em regressão, para duas funções em uso, a função perda é dada por:

$$
\begin{array}{r}
L(y, o)=L\left(y, f_{a}(\mathbf{x}, \mathbf{w})\right)=\left(y-f_{a}\right)^{2} \\
\text { ou o erro absoluto } \\
L(y, o)=L\left(y, f_{a}(\mathbf{x}, \mathbf{w})\right)=\left|y-f_{a}\right|
\end{array}
$$

Para classificação binária, 0 e 1, a função perda é:

$$
\begin{aligned}
& L(y, o)=0 \text { se } o=y \\
& L(y, o)=1 \text { se } o \neq y
\end{aligned}
$$

$o$ representa a saída de um algoritmo de aprendizado ou seja, $o=f_{a}(\mathbf{x}, \mathbf{w})$

Para a função aproximada pode-se usar qualquer estrutura matemática que mapeie entradas x em saída y. Assim, a função aproximada poderá ser um SVM. 


\subsubsection{Princípio de Minimização de Risco e Convergência Uni- forme}

Segundo Kecman (2001), aprendizado pode ser considerado como um problema de encontrar o melhor estimador $f$ usando dados disponíveis. Seguindo a apresentaçāo dos conceitos teóricos da minimização de risco para problemas de regressão, é mostrada uma explicação do porque e como resultados mudam quando todas as informações estão contidas somente nos dados de treinamento.

O Erro Médio ou Risco Esperado de um estimador $f$ representado pela Equação 4.2 , pode também ser escrito da seguinte forma:

$$
R[f]=\mathbb{E}\left[(y-f(\mathbf{x}))^{2}=\int(y-f(\mathbf{x}))^{2} P(\mathbf{x}, y), d \mathbf{x}, d y\right.
$$

O domínio do estimador $f$ é o espaço de função $\mathrm{T}$, e usando isso como precedente, o objetivo é encontrar o melhor elemento $f$ de $\mathbf{T}$ que minimize $R[f]$. Neste ponto, não há uma dependência explícita da função estimada $f(\mathbf{x})$ sobre os parâmetros de peso $\mathbf{w}$ que definem a relevância das features para aproximação de $f(\mathbf{x})$. Essas features relevantes são principalmente as propriedades geométricas do estimador $f(\mathbf{x})$. O melhor estimador $f(\mathbf{x}) \in T$ é procurado e conseqüentemente a função estimada é definida e analisada como uma função parametrrizada $f=f(\mathbf{x}, \mathbf{w})$ que agora depende do parâmetro peso $\mathbf{w}$.

O Risco Esperado dado pela Equação 4.5, pode agora ser decomposto como:

$$
R[f]=\mathbb{E}\left[\left(f_{o}(\mathbf{x})-f(\mathbf{x})\right)^{2}\right]+\mathbb{E}\left[\left(y-f_{o}(\mathbf{x})\right)^{2}\right]
$$

onde $(\mathbf{x}, y)$ são pares de dados e $f_{o}(\mathbf{x})$ é a função de regressão teórica. A Equaçāo 4.6 indica que a função de regressão minimiza o risco esperado em $\mathbf{T}$ e é o melhor estimador possível. Portanto:

$$
f_{o}(\mathbf{x})=\arg \min _{f \in T} R[f]
$$

A Equaçāo 4.7 indica que a função $f_{o}(\mathbf{x})$ é o argumento que pertence ao espaço de hipótese $T$ e que minimiza o risco $R[f]$. Na Equação 4.6 há duas partes, onde na primeira parte há uma dependência da escolha do estimador $f$ e não da saída do sistema y e a 
segunda, depende do ruído do sistema de saída y, e é este termo que limita a qualidade do estimador.

Para sistemas determinísticos, isto é, aqueles que são isentos de ruídos, situações em que $y=f_{o}(\mathbf{x})$, a função de probabilidade $P(y \mid \mathbf{x})$ é uma medida exata, portanto o segundo termo $\left(\mathbb{E}\left[\left(y-f_{o}(\mathbf{x})\right)^{2}\right]\right)$ é igual a zero. Quando $f_{1}=f_{o}(\mathbf{x}), \mathbb{E}\left[\left(y-f_{o}(\mathbf{x})\right)^{2}\right]$ é diferente de zero e este erro é devido a ruído, sendo igual a variância desse ruído.

Entretanto, há um problema em aplicar a Equação 4.5. A função de probabilidade $P(\mathbf{x}, y)$ é desconhecida e a distribuição livre do aprendizado deverá ter sua eficiência baseada somente nos pares dos dados de treinamento.

$\mathrm{O}$ algoritmo de aprendizado supervisionado embutido no experimento de Aprendizado de Máquina para aprender o relacionamento de entrada-saída $f_{o}(\mathbf{x})$ usando um conjunto de treinamento $D=\left\{[\mathbf{x}(i), y(i)] \in \Re^{n} \times \Re, i=1, \ldots, l\right\}$ contendo $l$ pares $\left(\mathbf{x}_{1}, y_{1}\right),\left(\mathbf{x}_{2}, y_{2}\right), \ldots,\left(\mathbf{x}_{l}, y_{l}\right)$, onde a entrada $\mathbf{x}$ são vetores $n$-dimensional $\mathbf{x} \in \Re^{n}$ e a resposta ao sistema $y \in \Re$ podem ser valores contínuos para tarefas de regressão ou discretos para problemas de classificação. O Risco Esperado $R[f]$ deve ser aproximado pelo Risco Empírico $\left(R_{\text {emp }}[f]\right)$ :

$$
R_{e m p}[f]=\frac{\sum_{i=1}^{l}\left(y_{i}-f\left(\mathbf{x}_{i}, \mathbf{w}\right)\right)^{2}}{l}
$$

Conseqüentemente, devido a $P(\mathbf{x}, y)$ ser desconhecida, o Princípio de Indução de Risco Empírico (ERM) substitui a média sobre $P(\mathbf{x}, y)$ por uma média sobre os exemplos de treinamento. A função estimada $f$, é agora expressa como uma função parametrizada que depende do parâmetro peso $\mathbf{w}-f=f(\mathbf{x}, \mathbf{w})$.

Segundo Kecman (2001) o Risco Empírico $R_{e m p}$ converge para o Risco Esperado quando a quantidade de dados tende a próximo do infinito $(l \rightarrow \infty)$ :

$$
\lim _{l \rightarrow \infty}\left(\left|R[f]-R_{e m p}[f]\right|\right)=0
$$

Esta regra é uma base teórica muito difundida para aplicações de estimadores com mínimos quadrado sabendo-se que a quantidade dos dados de treinamento é suficientemente grande. 
Entretanto a Equação 4.9 não garante que a função $f_{e m p}$ que minimiza o Risco Empírico $\left(R_{e m p}\right)$ converge para a melhor função $f$ que minimiza o Risco Esperado $R$. $O$ comando anterior aplica-se tão bem para os parâmetros $\mathbf{w}_{e m p}$ e $\mathbf{w}_{o}$ os quais definem as funções $f_{\text {emp }}$ e $f$, respectivamente. É necessário agora se ter uma convergência uniforme. Esta propriedade de consistência é definida:

$$
\lim _{l \rightarrow \infty} P\left[\left\{\sup _{\mathbf{w}}\left|R[\mathbf{w}]-R_{e m p}[\mathbf{w}]\right|>\epsilon\right\}\right]=0, \forall \epsilon>0
$$

onde P denota a probabilidade, e a Equação 4.10 mostra a convergência "na probabilidade". $R_{e m p}$ e $R$ denota o Risco Empírico e o Risco Esperado (verdadeiro) para um mesmo parâmetro $\mathbf{w}$.

A condição de consistência dada pela Equação 4.10, possui muitas propriedades teóricas interessantes e um dos resultados mais importantes é que a condição necessária e suficiente para uma taxa de convergência rápida e a consistência para distribuição independente de aprendizado ERM é que a dimensão VC de um conjunto de funções de aproximação é finito. O princípio ERM pode ser encontrado, em detalhes, em (Vapnik 1995). Desses, chega-se que para uma probabilidade $(1-\eta)$, as seguintes inequações são satisfeitas simultaneamente:

$$
\begin{array}{r}
R\left(\mathbf{w}_{e m p}\right)-R_{e m p}\left(\mathbf{w}_{e m p}\right)<\epsilon \\
R_{e m p}\left(\mathbf{w}_{o}\right)-R\left(\mathbf{w}_{o}\right)<\epsilon
\end{array}
$$

onde o peso $\mathbf{w}_{e m p}$ minimiza o Risco Empírico $R_{e m p}$, dado pela Equação 4.8 , e $\mathbf{w}_{o}$ minimiza o Risco Esperado dado pela Equação 4.6. Das últimas duas equações, têm-se que:

$$
R_{e m p}\left(\mathbf{w}_{e m p}\right) \leq R_{e m p}\left(\mathbf{w}_{o}\right)
$$

onde $\mathbf{w}_{e m p}$ e $\mathbf{w}_{o}$ são valores ótimos para os riscos correspondentes (significando que definem pontos de mínimo). Adicionando as Equações 4.12 e 4.12, e usando a 4.13, é obtida a seguinte equação:

$$
R\left(\mathbf{w}_{e m p}\right)-R\left(\mathbf{w}_{o}\right)<2 \epsilon
$$


Em outras palavras, o teorema de convergência uniforme exprimi que o vetor dos pesos $\mathbf{w}_{e m p}$ obtido pela minimização do Risco Empírico minimizará o Risco Esperado verdadeiro quando do crescimento do número de dados. Note que esta importante propriedade de convergência assegura que o conjunto de parâmetros que minimizam o Risco Empírico também minimiza o Risco Verdadeiro quando $l \rightarrow \infty$.

Há muitas outras funções de aproximação que minimizarão o Risco Empírico (erro de aproximação ou de treinamento), mas não necessariamente o Erro de Generalização (verdadeiro, esperado ou risco garantido). Isto é devido ao fato que uma máquina de aprendizado é treinada pelo uso de alguma amostra particular da função básica verdadeira, e conseqüientemente sempre produz funções de aproximação tendenciosas. Essas aproximações dependem necessariamente dos pares específicos dos dados de treinamento (amostras de treinamento).

A solução para estes problemas propostos na teoria de aprendizado estatístico é restringir os espaços de hipóteses $H$ de funções de aproximação a um conjunto menor que o espaço de função $T$, os quais simultaneamente controlam a complexidade das funções de aproximação. Os modelos usados são parametrizados, e com um aumento do número de parâmetros, eles formam uma estrutura aninhada da seguinte forma: $H_{1} \subset H_{2} \subset H_{3} \subset$ $\ldots \subset H_{n-1} \subset H_{n} \subset \ldots \subset H($ Kecman 2001).

Portanto, neste conjunto aninhado de funções, cada função sempre contém a função anterior, menos complexa, como pode ser visto na Figura 4.5. Tipicamente, $H_{n}$ pode ser um conjunto de polinômios em uma variável de grau $n-1$. Minimizar $R_{\text {emp }}$ sobre um conjunto $H_{n}$, aproxima a função de regressão $f_{o}$ pela função

$$
\hat{\mathrm{f}}_{n, l} \equiv \arg \min _{f \in H_{n}} R_{e m p}|f|
$$

SVMs podem ser representados como combinações lineares de funções básicas fixadas em $f_{a}(\mathbf{x}, \mathbf{w})=\sum_{\alpha=1}^{n} w_{\alpha} \varphi_{\alpha}(\mathbf{x})$. Para modelos lineares, como é o caso anterior, a dimensão VC é igual a $h$, que define a complexidade e a capacidade das funções de aproximação, que é igual a $n+1$. Esta é uma propriedade atrativa para modelos lineares.

Para esses modelos, a Equação 4.15 pode ser reescrita: 


$$
\hat{\mathrm{f}}_{n, l} \equiv \arg \min _{w_{\alpha}} R_{e m p}\left|f_{i}\right|
$$

Ao invés de minimizar o risco esperado estimando a função de regressão $f_{o}$ sobre um grande espaço de hipóteses $\mathbf{T}$, a função $\hat{\mathrm{f}}_{n, l}$ é obtida minimizando o risco empírico sobre um pequeno conjunto de funções $H_{n}$. Conseqüentemente, sempre haverá um erro de generalização $\left(e_{g e n}\right)$, o qual pode ser decomposto em 2 partes, como mostrado na seguinte equação:

$$
e_{g e n}=e_{a p p}+e_{e s t}
$$

É mostrado na Figura 4.5 o vetor soma das 2 possiveis fontes de erros dos dados na tarefa de aprendizado. Pode-se também visualizar que a medida que a quantidade de dados cresce, cresce o Erro Estimado $\left(e_{e s t}\right)$ e diminue o Erro Aproximado $\left(e_{a p p}\right)$.

A primeira fonte de erro é tentar aproximar a função $f_{0}$, que é uma estrutura de dimensão infinita em $\mathrm{T}$, com a função $f_{n} \in H_{n}$, que é parametrizada em um número finito de parâmetros. Este é um Erro Aproximado $\left(e_{a p p}\right)$, o qual pode ser medido como a distância da melhor função $f_{n}$ em $H_{n}$ e a função de regressão $f_{o}$, e é definido como o valor esperado da distância euclidiana. Tem-se, então:

$$
e_{a p p}=E\left[\left(f_{o}-f_{n}\right)^{2}\right]=R\left[f_{n}\right]-R\left[f_{o}\right]
$$

O Erro Aproximado $\left(e_{a p p}\right)$ depende da capacidade de aproximação na escolha da classe de hipóteses $H_{n}$ e não do conjunto de dados de treinamento.

Já o Erro de Risco Empírico $\left(R_{\text {emp }}\right)$ é minimizado usando um conjunto de dados de treinamentos finito e geralmente esparço. Tal aprendizado resulta em $\hat{\mathrm{f}}_{n, l} \in H_{n}$, que é a melhor função de aproximação para um particular conjunto de dados de treinamento. A função aproximada $\hat{\mathrm{f}}_{n, l}$ será mais eficiente, a medida que o número de dados de treinamento $l$ aumentar. De acordo com o teorema de convergência uniforme, a medida que $l$ aumenta, uma estimativa do Risco Esperado, a função $\hat{\mathrm{f}}_{n, l}$ melhora e o Risco Empírico $\left(R_{e m p}\right)$ converge para o Risco Esperado $(\mathrm{R})$.

A medida da diferença entre esses dois riscos é definida como Erro Estimado $\left(e_{e s t}\right)$ : 


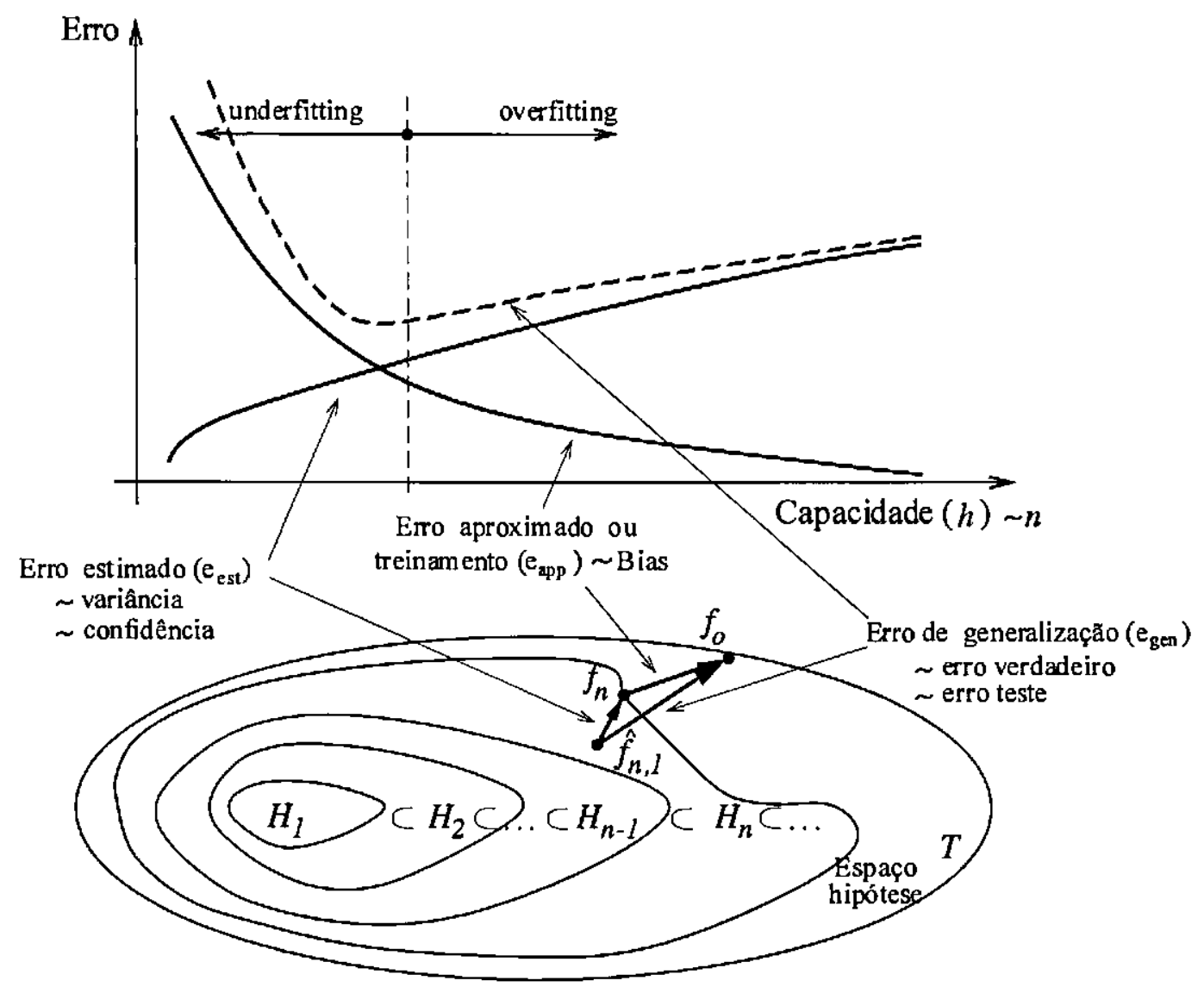

Figura 4.5: Estrutura de funções de hipóteses e diferentes erros que dependem do número de funções básicas $n$, para um número fixo de exemplos $l$ (Kecman 2001)

$$
e_{e s t}=\left|R_{e m p}[f]-R[f]\right|
$$

Vapnik (1995) demonstrou que o limite do erro estimado na seguinte equação é válido com uma probabilidade $1-\eta$, resultando:

$$
e_{e s t}=\left|R_{e m p}[f]-R[f]\right| \leq \Omega(l, n, \eta), \forall f \in H_{n}
$$

A fórmula particular $\Omega(l, n, \eta)$ depende de um conjunto de problemas, mas é geralmente uma função decrescente de amostras de tamanho $l$ e uma função crescente do número $n$ de parâmetros livres da função aproximada. 


\subsubsection{Dimensão Vapnik-Chervonenkis}

A Dimensão Vapnik-Chervonenkis (VC) $h$ é uma propriedade de um conjunto de funções de aproximação de Aprendizado de Máquina que é usado em vários resultados importantes na teoria de aprendizado estatístico.

Essa propriedade das funções $f(\mathbf{x}, \mathbf{w}) \rightarrow\{-1,1\}$, VC, indica o número máximo ( $l$ ) de pontos que podem ser divididos de todos os $2^{l}$ modos, utilizando esse conjunto de funções. Usa-se w como um conjunto genérico de parâmetros: a escolha de $\mathbf{w}$ especifica uma função particular.

Somente serão consideradas as funções que correspondem ao caso de reconhecimento de padrões de duas classes, onde $f(\mathbf{x}, \mathbf{w}) \in\{-1,1\} \forall \mathbf{x}, \mathbf{w}$.

A dimensão VC para um conjunto de funções é definida com o número máximo de pontos de treinamento que podem ser separados por $f(\mathbf{x}, \mathbf{w})$. Se a dimensão de VC é $h$, então existe no mínimo um conjunto de $h$ pontos que podem ser separados. A visualização da dimensão de VC para um conjunto de linhas orientadas em $R^{2}$, como sendo relação com h igual a 3 , é ilustrado na Figura 4.6.

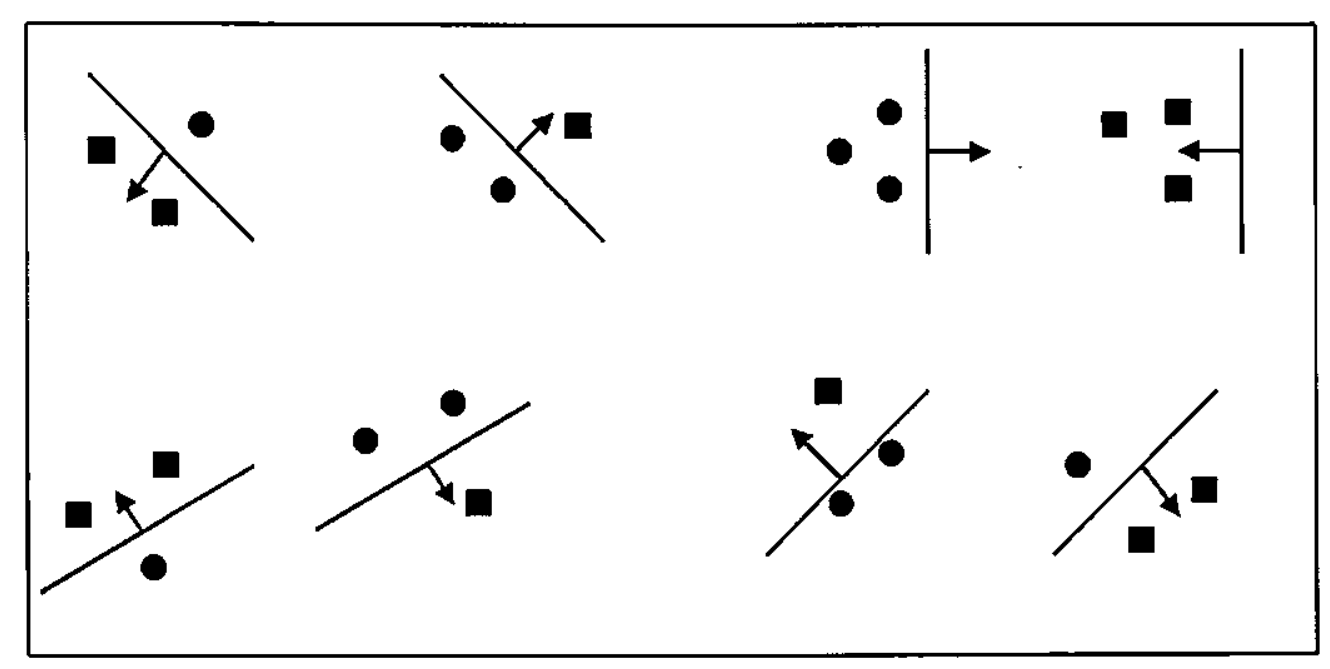

Figura 4.6: Dimensão VC (três pontós em $R^{2}$ separados e todos os possíveis $2^{3}=8$ modos $(\mathrm{h}=3))$ 


\subsubsection{Minimização de Risco Estrutural}

Minimização de Risco Estrutural (SRM) é um princípio indutivo para aprendizado sobre conjuntos de dados de treinamento finito. A idéia básica de SRM é escolher, dentre um grande número de modelos candidatos, um modelo de alta complexidade para descrever os pares de dados de treinamento. Como visto anteriormente, isto pode ser feito restringindo o espaço de hipóteses $H$ das funções de aproximação e simultaneamente controlar sua complexidade. Os modelos de Aprendizado de Máquina podem ser parametrizados, pelo incremento do número de parâmetros (chamado aqui de pesos w), formando uma nova estrutura como segue: $H_{1} \subset H_{2} \subset H_{3} \subset \ldots \subset H_{n-1} \subset H_{n} \subset \ldots \subset H$ (Kecman 2001). Neste conjunto aninhado de funções, cada função sempre contém a função anterior, menos complexa, como observado na Figura 4.5. $H_{n}$ pode ser, por exemplo, um conjunto de polinômios em uma variável de grau $n$.

A meta do aprendizado é conseguir selecionar subconjuntos, os quais combinem complexidade dos dados de treinamento com capacidade do modelo aproximado.

A escolha da capacidade do modelo ótimo garante a minimização do Risco Esperado (erro de generalização) da seguinte forma. Há vários limites para generalização para uma implementação de Aprendizado de Máquina ERM que analiticamente conecta Erro de Generalização $R\left(\mathbf{w}_{n}\right)$, Erro Aproximado $R_{e m p}\left(\mathbf{w}_{n}\right)$, dimensão VC $h$, número de amostras de treinamento $l$, e probabilidade (ou nível de confidência) 1 - $\eta$ para todas as funçōes de aproximação para classificação binária ou regressão. A minimização desses limites é a essência da Minimização do Risco Estrutural.

O limite para generalização para um classificador binário é dado pela Equação 4.21 com a probabilidade de ao menos $1-\eta$ para todas as funções de aproximação (pesos $\mathbf{w}_{n}$ que definem essas funçōes) incluindo a função que minimiza o Risco Empírico:

$$
R\left(\mathbf{w}_{n}\right) \leq R_{e m p}\left(\mathbf{w}_{n}\right)+\Omega\left(\frac{h}{l}, \frac{\ln \eta}{l}\right)
$$

o segundo termo do lado direito é chamado de Confidência VC (termo de confidência ou intervalo de confidência), definido como:

$$
\Omega\left(\frac{h}{l}, \frac{\ln \eta}{l}\right)=\sqrt{\frac{h\left(\ln \left(\frac{2 l}{h}+1\right)-\ln \left(\frac{\eta}{4}\right)\right.}{l}}
$$


Em (Kecman 2001) é mostrado que quando a dimensão de VC do modelo é baixa, o Erro Esperado também é baixo, o que significa uma boa generalização. Esta propriedade é de particular interesse no campo computacional, porque o modelo que generaliza bem é um bom modelo, e não o modelo que tem uma boa eficiência nos dados de treinamento. Boa eficiência nos dados de treinamento é necessária, mas condição insuficiente para um bom modelo.

\subsection{Classificação usando Vetores de Suporte - Casos Lineares}

Considere um problema de classificação que consiste em separar um conjunto de pontos $\left(\mathbf{x}_{1}, y_{1}\right),\left(\mathbf{x}_{2}, y_{2}\right), \ldots\left(\mathbf{x}_{l}, y_{l}\right)$, com $\mathbf{x} \in \Re^{n}, y \in\{+1,-1\}$, através de um hiperplano $w \cdot x+$ $b=0$. Segundo Burges (1998), pode-se dizer que existe um hiperplano ótimo, quando é possível separar o conjunto de pontos sem erros, com a margem entre os vetores de suporte e o hiperplano sendo máxima. Usando um exemplo dado para treinamento durante o estágio de aprendizado, encontra-se os parâmetros específicos de pesos $\mathbf{w}=\left[\begin{array}{lll}w_{1} & w_{2}\end{array} \ldots\right.$ $\left.w_{n}\right]^{T}$ e um bias $b$ :

$$
\begin{array}{ll}
\left(\mathbf{w}^{\mathbf{T}} \cdot \mathbf{x}_{i}\right)+b \geq 0, & \text { se } y_{i}=1 \\
\left(\mathbf{w}^{\mathbf{T}} \cdot \mathbf{x}_{i}\right)+b<0, & \text { se } y_{i}=-1
\end{array}
$$

Para descrever o hiperplano de separação usa-se a seguinte forma canônica:

$$
\begin{aligned}
\left(\mathbf{w}^{\mathbf{T}} \cdot \mathbf{x}_{i}\right)+b & \geq+1, \quad \text { se } y_{i}=1 \\
\left(\mathbf{w}^{\mathbf{T}} \cdot \mathbf{x}_{i}\right)+b & \leq-1, \quad \text { se } y_{i}=-1 \\
\text { isto é } & \\
y_{i}\left[\left(\mathbf{w}^{\mathbf{T}} \cdot \mathbf{x}_{i}\right)+b\right]-1 & \geq 0, i=1,2, \ldots l
\end{aligned}
$$

Assim, para cada par de valores $(w, b)$ que satisfaçam as condições da equação 4.26, existem hiperplanos separadores $H_{1}$ e $H_{2}$ dos pontos, conforme ilustrado na Figura 


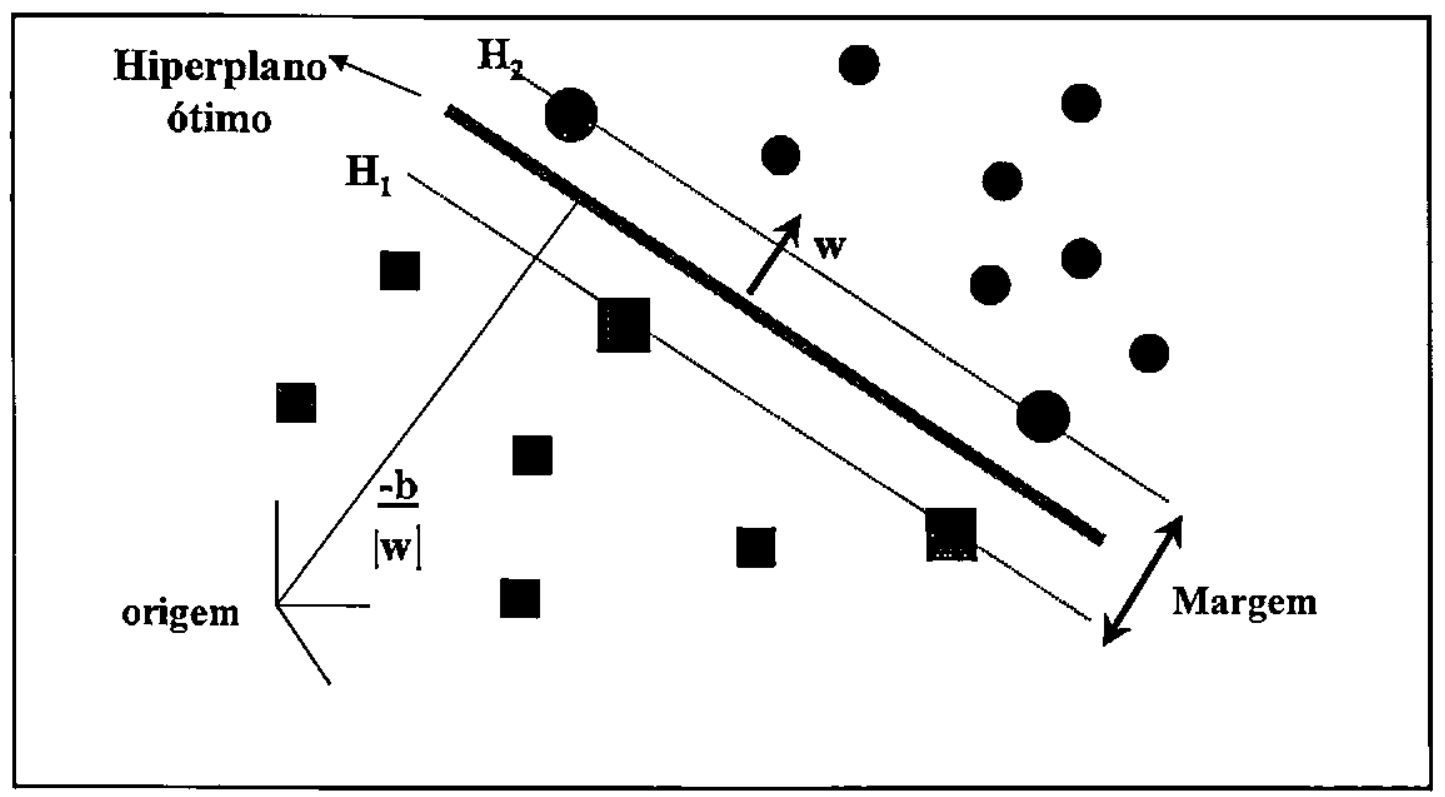

Figura 4.7: Hiperplano de separação ótimo (aquele que separa os dados com uma margem máxima)

4.7. Considerando que existam pontos que satisfaçam a equação 4.24, esses pontos se encontram no hiperplano $H_{1}: \mathbf{w}^{\mathbf{T}} \cdot \mathbf{x}_{i}+b=1$ com a norma w e distância perpendicular da origem $\frac{|I-b|}{\|\mathbf{w}\|}$.

Similarmente, há pontos nos quais a igualdade da equação 4.25 é satisfeita e esses pontos se encontram no hiperplano $H_{2}: \mathbf{w}^{\mathrm{T}} \cdot \mathbf{x}_{i}+b=-1$ com a norma $\mathbf{w}$ e distância perpendicular da origem $\frac{|-1-b|}{\|\mathbf{w}\|}$.

O ideal é encontrar, entre os hiperplanos separadores, aquele que consegue dividir as classes de forma que a distância dos pontos mais próximos do hiperplano até o próprio hiperplano seja a maior possível. Daí surge o conceito de margem, que é a soma da distância do ponto da classe positiva mais próxima do hiperplano com a distância do ponto da classe negativa mais próxima do hiperplano. Sabendo que a distância de um ponto $\mathbf{x}$ a um hiperplano $(\mathbf{w}, \mathbf{b})$ é $d(w, b ; x)=\frac{\left|\mathbf{w}^{\mathbf{T}} \cdot \mathbf{x}+b\right|}{\|\mathbf{w}\|}$, pode-se calcular a margem da seguinte forma:

$$
\rho(w, b)=\min _{\left\{x_{i} ; y_{i}=1\right\}} d\left(w, b ; x_{i}\right)+\min _{\left\{x_{j}: y_{j}=-1\right\}} d\left(w, b ; x_{j}\right)
$$


das restrições 4.24 e 4.25 , temos que:

$$
\begin{aligned}
& =\min _{\left\{x_{i}: y_{i}=1\right\}} \frac{\left|w^{T} \cdot x_{i}+b\right|}{\|w\|}+\min _{\left\{x_{j}: y_{j}=-1\right\}} \frac{\left|w^{T} \cdot x_{j}+b\right|}{\|w\|} \\
& =\frac{1}{\|w\|}\left(\min _{\left\{x_{i}: y_{i}=1\right\}}\left|w^{T} \cdot x_{i}+b\right|+\min _{\left\{x_{j}: y_{j}=-1\right\}}\left|w^{T} \cdot x_{j}+b\right|\right)
\end{aligned}
$$

$$
\begin{aligned}
& =\frac{1}{\|w\|}(|1|+|-1|) \\
\rho(w, b) & =\frac{2}{\|w\|}
\end{aligned}
$$

A Equação 4.27 representa um resultado muito interessante. A minimização de $\|\mathbf{w}\|$, causará a maximização da margem. Para $\|\mathbf{w}\|=\sqrt{(\mathbf{w} \mathbf{w})}=\sqrt{w_{1}^{2}+w_{2}^{2}+\ldots+w_{n}^{2}}$. A função $\sqrt{f}$ é monotônica, e minimizar $\sqrt{f}$ significa minimizar $f$. Conseqüentemente minimizar $\|\mathbf{w}\|$ é igual a minimizar $\mathbf{w}^{\mathbf{T}} \cdot \mathbf{w}=(\mathbf{w} \mathbf{w})=\sum_{i=1}^{n} w_{i}^{2}=w_{1}^{2}+w_{2}^{2}+\ldots+w_{n}^{2}$.

Portanto, a fim de encontrar o hiperplano separador ótimo que tenha uma máxima margem, tem-se que minimizar $\|w\|^{2}$ sujeito as condições da Equação 4.26. Este é um problema clássico de otimização não linear. Tal problema é solucionado introduzindo multiplicadores de Lagrange positivos $\alpha_{i}, i=1, \ldots l$, um para cada restrição da equação 4.26. Assim:

$$
L(w, b, \alpha)=\frac{1}{2} \mathbf{w}^{\mathbf{T}} \cdot \mathbf{w}-\sum_{i=1}^{l} \alpha_{i}\left\{y_{i}\left[\left(\mathbf{w}^{\mathbf{T}} \cdot \mathbf{x}_{i}\right)+b\right]-1\right\}
$$

A equação 4.28 deve ser minimizada em relação a w e $b$ e maximizada em relação a $\alpha_{i} \geq 0$. Este problema pode ser resolvido no espaço primal (que é o espaço dos parâmetros w e $b$ ) ou no espaço dual (que é o espaço dos multiplicadores de Lagrange $\alpha_{i}$ ). A dualidade Lagrangiana Clássica permite transformar o problema primal da equação 4.28 em um problema dual, este de mais fácil resolução. Ambas, as funções objetivas, da equação 4.28 e as condições da equação 4.26, são funções convexas e as condições Karush-Kuhn-Tucker (KKT) são necessárias e suficientes para encontrar o máximo da equação 4.28 (Kecman 2001). As condições KKT são as seguintes. Para um ponto $\left(\mathbf{w}_{0}, b_{0}, \alpha_{0}\right)$, a derivada da Lagrangiana L, tem-se: 


$$
\begin{aligned}
& \frac{\partial L\left(w_{0}, b_{0}, \alpha\right)}{\partial b_{0}}=0 \Rightarrow \sum_{i=1}^{l} \alpha_{i} y_{i}=0 \\
& \frac{\partial L\left(w_{0}, b_{0}, \alpha\right)}{\partial w_{0}}=0 \Rightarrow w=\sum_{i=1}^{l} \alpha_{i} \mathbf{x}_{i} \cdot y_{i}
\end{aligned}
$$

As condições complementares devem também ser satisfeitas:

$$
\alpha_{i}\left\{y_{i}\left[\mathbf{w}^{\mathbf{T}} \cdot \mathbf{x}_{i}+b\right]-1\right\}=0, i=1, \ldots, l
$$

Substituindo as Equações 4.29 e 4.30 na Equação 4.28, Lagrangiana primal , obtémse a Lagrangiana dual, dada pela seguinte equação:

$$
L_{d}(\alpha)=\sum_{i=1}^{l} \alpha_{i}-\frac{1}{2} \sum_{i, j=1}^{l} \alpha_{i} \alpha_{j} y_{i} y_{j}\left(\mathbf{x}_{i}^{T} \cdot \mathbf{x}_{j}\right)
$$

sujeito às condições:

$$
\begin{array}{r}
\alpha_{i} \geq 0 \quad i=1, \ldots, l \\
\sum_{i=1}^{l} \alpha_{i} y_{i}=0
\end{array}
$$

Note que a Lagrangiana Dual $L_{d}(\alpha)$ é expressa em termos dos dados de treinamento e depende somente do produto escalar interno $\mathbf{x}_{i} \cdot \mathbf{x}_{j}$. Note também que o número de variáveis desconhecidas é igual ao número dos dados de treinamento $l$. Depois do aprendizado, o número de parâmetros livres é igual ao número de Vetores de Suporte, e não depende mais da dimensionalidade do espaço de entrada.

Soluções $\alpha_{i}$ para problema de otimização dual determina os parâmetros $\mathbf{w}_{\mathrm{o}}$ e $b_{o}$ do hiperplano ótimo, utilizando-se das Equações 4.29 e 4.31, obtendo:

$$
\mathbf{w}_{\mathbf{o}}=\sum_{i=1}^{l} \alpha_{o} y_{i} \mathbf{w}_{\mathbf{i}}, i=1, \ldots, l
$$


onde $N_{V S}$ é o número de vetores de suporte. Note que um vetor peso ótimo $\mathbf{w}_{\mathrm{o}}$, assim como o termo bias $b_{o}$, é calculado usando somente vetores de suporte, apesar do fato que o somatório feito pela Equação 4.34 ser feito sobre todos os dados de treinamento. Isso só é possível devido ao fato que todos os multiplicadores de Lagrange para qualquer vetor que não seja o vetor de suporte é zero $\left(\alpha_{o i}=0, i=N_{V S}+1, l\right)$.

Finalmente tendo calculado $w_{0}$ e $b_{o}$, obtém-se um hiplerplano de decisão $d(\mathbf{x})$ e uma função indicator $i_{F}=o=\operatorname{sign}(d(\mathbf{x}))$ :

$$
d(\mathbf{x})=\sum_{i=1}^{l} w_{o i} x_{i}+b_{o}=\sum_{i=1}^{l} y_{i} \alpha_{i} \mathbf{x}^{\mathrm{T}} \cdot \mathbf{x}_{\mathbf{i}}+b_{o}
$$

Padrões de dados de treinamento que tem multiplicadores de Lagrange diferentes de zero são chamados de vetores de suporte (VS). Se os dados säo linearmente separáveis, todos os vetores de suporte se encontram na margem, e são geralmente uma pequena porção do conjunto de treinamento $\left(N_{V S} \ll l\right)$.

\subsection{Generalização para Problemas não Lineares}

Os problemas citados foram resolvidos através de um hiperplano separador linear. Todavia, muitos problemas exigem uma solução não linear. O que se faz nesse caso é mapear o espaço de entrada em um espaço hiper-dimensional de atributos e obter hiperplanos separadores da mesma forma já descrita para os lineares.

Uma forma de projetar SVMs não lineares é mapear vetores de entrada $\mathrm{x} \in \Re^{\mathbf{n}}$ em vetores $\mathbf{z}$ de alta dimensão de espaço de features $\mathrm{F}(\mathbf{z}=\boldsymbol{\Phi}(\mathbf{x})$, onde $\Phi$ representa um mapeamento $\Re^{n} \rightarrow \Re^{f}$ ), e resolver um problema de classificação linear no espaço de features:

$$
x \in \Re^{n} \rightarrow \mathbf{z}(\mathbf{x})=\left[a_{1} \phi_{1}(\mathbf{x}), a_{2} \phi_{2}(\mathbf{x}), \ldots, a_{n} \phi_{n}(\mathbf{x})\right]^{T} \in \Re^{f}
$$

A solução para uma função indicator $i_{F}=\operatorname{sign}\left(\mathbf{w}^{\mathbf{T}} \mathbf{z}(\mathbf{x})+b\right)$, a qual é um classificador linear em um espaço de feature $\mathbf{F}$, é dado pela seguinte equação: 


$$
i_{F}(\mathbf{x})=\operatorname{sign}\left(\sum_{i=1}^{l} y_{i} \alpha_{i} \mathbf{z}^{T}(\mathbf{x}) \mathbf{z}\left(\mathbf{x}_{\mathbf{i}}\right)+b\right)
$$

A explosão na dimensionalidade pode ser evitada, uma vez que os dados de treinamento só aparecem na forma de produto escalar $\mathbf{x}_{i}^{T} \cdot \mathbf{x}_{j}$. Esses produtos são recalculados pelo produto escalar $\mathbf{z}^{T} \mathbf{z}_{i}=\left[\phi_{1}(\mathbf{x}), \phi_{2}(\mathbf{x}), \ldots, \phi_{n}(\mathbf{x})\right]\left[\phi_{1}\left(\mathbf{x}_{i}\right), \phi_{2}\left(\mathbf{x}_{i}\right), \ldots, \phi_{n}\left(\mathbf{x}_{i}\right)\right]^{T}$ no espaço de features $\mathrm{F}$, e mais tarde é expresso usando a função Kernel:

$$
K\left(\mathbf{x}_{\mathbf{i}}, \mathbf{x}_{\mathbf{j}}\right)=\mathbf{z}_{\mathbf{i}}^{\mathbf{T}} \mathbf{z}_{\mathbf{j}}=\Phi^{\mathbf{T}}\left(\mathbf{x}_{\mathbf{i}}\right) \Phi\left(\mathbf{x}_{\mathbf{j}}\right)
$$

A função kernel $K\left(\mathbf{x}_{i}, \mathbf{x}_{j}\right)$ é uma função do espaço de entrada. Entretanto, o produto escalar no espaço de features $\boldsymbol{\Phi}^{\mathbf{T}}\left(\mathbf{x}_{\mathrm{i}}\right) \boldsymbol{\Phi}\left(\mathrm{x}_{\mathrm{j}}\right)$ são calculados diretamente por Kernels $K\left(\mathbf{x}_{i}, \mathbf{x}_{j}\right)$ para vetores de dados de treinamento em um espaço de entrada. Portanto, usando um Kernel escolhido, um SVM pode ser construído e atuar num espaço dimensional infinito. As funções Kernels mais utilizadas, para classificação e em especial para Text Mining serão descritas a seguir.

O algoritmo de aprendizado para um classificador SVM não linear segue o projeto da descoberta de um hiperplano ótimo num espaço de features. Agora para um espaço z-espaço, a Lagrangiana dual dada pela Equação 4.40, fica:

$$
L_{d}(\alpha)=\sum_{i=1}^{l} \alpha_{i}-\frac{1}{2} \sum_{i, j=1}^{l} \alpha_{i} \alpha_{j} y_{i} y_{j}\left(\mathbf{z}_{i}^{T} \cdot \mathbf{z}_{j}\right)
$$

e usando a Equação 4.39, pela escolha de um Kernel, a maximização da Lagrangiana, resulta na seguinte equação:

$$
L_{d}(\alpha)=\sum_{i=1}^{l} \alpha_{i}-\frac{1}{2} \sum_{i, j=1}^{l} \alpha_{i} \alpha_{j} y_{i} y_{j} K\left(\mathbf{x}_{i}, \mathbf{x}_{j}\right)
$$

sujeito às condições:

$$
\begin{array}{r}
\alpha_{i} \geq 0 \quad i=1, \ldots, l \\
\sum_{i=1}^{l} \alpha_{i} y_{i}=0
\end{array}
$$

A hiper-superfície de decisāo $d(\mathbf{x})$ é determinada por: 


$$
d(\mathbf{x})=\sum_{i=1}^{l} y_{i} \alpha_{i} K\left(\mathbf{x}, \mathbf{x}_{\mathbf{i}}\right)+b
$$

A função indicator dada pela Equação 4.44, que é geralmente também uma hipersuperfície para $n>3$, definirá o classificador para vetores de suporte (VS) não lineares.

$$
i_{F}(\mathbf{x})=\operatorname{sign}(d(\mathbf{x}))=\operatorname{sign}\left(\sum_{i=1}^{l} y_{i} \alpha_{i} K\left(\mathbf{x}, \mathbf{x}_{\mathbf{i}}\right)+b\right)
$$

Entretanto, muitas vezes o termo bias pode ser acomodado dentro da função $\mathrm{Ker}$ nel, fazendo parte implícita da função Kernel, podendo definir entāo classificador para vetores de suporte (VS) não lineares, como:

$$
i_{F}(\mathbf{x})=\operatorname{sign}\left(\sum_{s=1}^{n r o V S_{s}} y_{s} \alpha_{s} K\left(\mathbf{x}, \mathbf{x}_{\mathbf{s}}\right)\right)
$$

sujeita às mesmas restrições de 4.33. A função $\operatorname{Kernel}(\mathbf{K})$, faz o mapeamento dos vetores para o espaço de atributos, encontrando uma solução não-linear para o problema.

\section{Funções Kernel para SVM}

As funções Kernel (Gunn 1998) são funções que devem fazer o mapeamento do espaço hiper-dimensional em um plano da dimensão da entrada, de modo que as operações possam ser realizadas utilizando o plano de entrada. Para se ter uma função Kernel para mapeamento de uma espaço hiper-dimensional deve-se encontrar uma função que mapeie o produto interno desse espaço, isto é:

$$
K(x, y)=k(x) \cdot k(y)
$$

Algumas funções Kernel para alguns dos tipos de problemas comumente encontrados para reconhecimento de padrões são:

- Função Polinomial: método muito utilizado para problemas não lineares, cujo grau do polinômio é $d$.

$$
K(x, y)=(x . y)^{d}, d=1,2, \ldots \text { ou } K(x, y)=((x . y)+1)^{d}
$$


O segundo Kernel é utilizado para evitar problemas quando $x . y=0$

- Função Radial-Basis Gaussiana

$$
K(x, y)=\exp \left(-\frac{(x-y)^{2}}{2 \sigma^{2}}\right)
$$

- Função Radial-Basis Exponencial: pode ser uma função atrativa quando descontinuidades são aceitáveis

$$
K(x, y)=-\exp \left(-\frac{|x-y|}{2 \sigma^{2}}\right)
$$

- Perceptron Multi-Camadas

$$
K(x, y)=-\tanh (\operatorname{scale}(x . y)-o f f s e t)
$$

É uma função kernel válida para certos parâmetros de scale e offset. Os vetores de suporte correspondem a primeira camada e os multiplicadores de Lagrange correspondem ao peso.

- Série de Fourier

$$
K(x, y)=\frac{\sin \left(N+\frac{1}{2}\right)(x-y)}{\sin \left(\frac{1}{2}(x-y)\right)}
$$

\subsection{Considerações Finais}

Neste capítulo foi visto o algoritmo de Support Vector Machines um dos algoritmos que tem sido muito utilizado para categorização de textos e por sua origem estatística foi revisto alguns conceitos ligados ao SVM.

Cooley (1999) fez uma comparação entre os algoritmos SVM e Vizinho Mais Próximo e observou que o melhor resultado é encontrado para SVM sem reduções de features.

Yang \& Liu (1999) fez comparações entre 5 algoritmos para categorização de textos: SVM, Vizinho Mais Próximo, Naive Bayes, Rede Neural (NNet) e LLSF. E em todos os testes realizados, usando $F_{1}$ para comparação, tem-se que SVM e Vizinho Mais Próximo possuem uma melhor eficiência, quando comparado com os outros algoritmos. 
Drucker, Wu, \& Vapnik (1999) fez comparações entre 4 algoritmos para categorização de textos com a finalidade de identificar emails spam. Os algoritmos foram: Ripper, Rocchio, Árvore de Decisão - Boosting e SVM. SVM e Árvore de Decisão - Boosting são os melhores candidatos para uma melhor eficiência, sendo que para Ripper há uma taxa de erro um pouco menor, mas a dispersão de erros é melhor para o SVM.

Joachims (1998) fez experimento similar ao anterior, e comparou 5 algoritmos de categorização: Naive Bayes, Rocchio, Vizinho Mais Próximo, C4.5 e SVM. Usando todas as features o melhor desempenho foi obtido com o algoritmo SVM.

Dumais, Platt, Heckerman, \& Sahami (1998) fez comparações entre 5 algoritmos para categorização de textos: Rocchio, Árvore de Decisão, Naive Bayes, Redes Bayes e SVM. Foram usadas as medidas de precisão e cobertura. A precisão do algoritmo SVM foi a melhor apresentada.

No problema de classificação efetua-se a predição de um atributo-meta categórico de um exemplo mediante o conhecimento de outros atributos relacionados ao exemplo. $O$ modelo gerado mapeia, de alguma forma, o conjunto de entrada, formado pelos atributos conhecidos, em uma saída, que indica a classe predita do exemplo. Por motivos de simplificação, ao invés de indicar diretamente a classe em que pertencem, muitos algoritmos simplesmente criam modelos que denotam ausência ou presença na classe. Apesar dessa simplificação é possível resolver problemas com multi-classes, agrupando os modelos feitos para cada uma das classes. Para o SVM o problema multi-classe é tratado em (Lee, Lin, \& Wahba 2001; J.Weston \& Watkins 1998; Hsu \& Lin 2001).

No próximo capítulo é encontrada uma descrição do problema tratado neste trabalho, bem como dos dados empregados nas investigações e o planejamento dos experimentos realizados. 


\section{Capítulo 5}

\section{Experimentos}

\subsection{Considerações Iniciais}

Nos capítulos anteriores for am revistos alguns conceitos relacionados a Text Mining, as principais etapas desse processo, bem como algumas técnicas e algoritmos que podem ser usados. Como citado, o processo de Text Mining pode ser dividido em Coleta de Dados, Pré-processamento, Extração de Conhecimento e Avaliação e Interpretação dos Resultados.

O objetivo principal deste trabalho é pesquisar e analisar alguns métodos computacionais usados para extrair conhecimento de textos. Como as duas primeiras etapas do Processo de Text Mining, a Coleta de Documentos e o Pré-processamento, foram explorados em (Imamura 2001), neste trabalho os esforços foram concentrados na Extração de Conhecimento e Avaliação dos Resultados.

Na Seção 5.2 é apresentada uma visão geral do módulo de Pré-processamento de textos e na Seção 5.3 é mostrado todo o planejamento dos experimentos.

\subsection{Módulo de Pré-processamento de Textos}

O módulo de Pré-processamento é constituído de seis scritps para a identificação de atributos, seis scripts para atribuiçãa de pesos, que são necessários para a construção da representação e um script de redução de representação. 
Para construção dos scripts para identificação de atributos foram utilizados três algoritmos baseados em (Mladenić 1998; Witten, Paynter, Frank, Gutwin, \& NevillManning 1999; Weiss \& Indurkhya 1998). Esses algoritmos utilizam diferentes estratégias para considerar quais os termos que constituirão os atributos. $\mathrm{O}$ algoritmo baseado em (Mladenić 1998) considera que os atributos são formados por até cinco termos que não sejam stopwords e que tenham ocorrido pelo menos quatro vezes no conjunto de documentos. No algoritmo baseado em (Witten, Paynter, Frank, Gutwin, \& Nevill-Manning 1999), os atributos são formados por até três termos, sendo que o primeiro e o último não podem ser stopwords (palavras irrelevantes). E no algoritmo baseado em (Weiss \& Indurkhya 1998), os atributos são formados por um único termo que não seja stopword. Para identificação das stopwords foi utilizada uma lista contendo 613 palavras (Imamura 2001).

Para cada um dos três algoritmos citados anteriormente, considerou-se o uso de uma estratégia para a realização da normalização dos termos, utilizando-se de duas ferramentas: a primeira é o stemming, baseada no algoritmo de Porter (Porter 1980) e a segunda é a transformação na forma canônica, baseada na análise léxica e sintática, fazendo-se uso da ferramenta chamada Etiquetador, cedida pelo NILC ${ }^{1}$ (Nunes 1996).

Os scripts desenvolvidos por Imamura (2001) para identificação de atributos estão ilustrados na Figura 5.1 e descritos a seguir.

O script ScpDCan, baseado em (Mladenić 1998), utiliza um algoritmo para encontrar os atributos compostos, formado por uma seqüência de até 5-grams (gram é um termo que não é uma stopword). Nesse algoritmo iterativo, cada $i$-gram é gerado no $i$-ésimo passo de iteração com base apenas nos grams de tamanho $i-1$ gerados na iteração anterior. $\mathrm{Na}$ primeira iteração são encontrados os atributos simples, que não sejam stopwords e que ocorram mais de três vezes no documento. A partir desses, são gerados os 2-grams e o algoritmo processa as iterações até, no máximo, os 5-grams serem todos construídos. Dessa forma os atributos designados com 1-gram referem-se aos atributos simples e qualquer valor de $n>1$ refere-se a atributos compostos, que foram agrupados. Embora o uso de termos compostos seja interessante, o processo para gerá-los pode ser bastante

\footnotetext{
${ }^{1}$ Núcleo Interinstitucional de Lingüistica Computacional: http://nilc.icmc.usp.br
} 


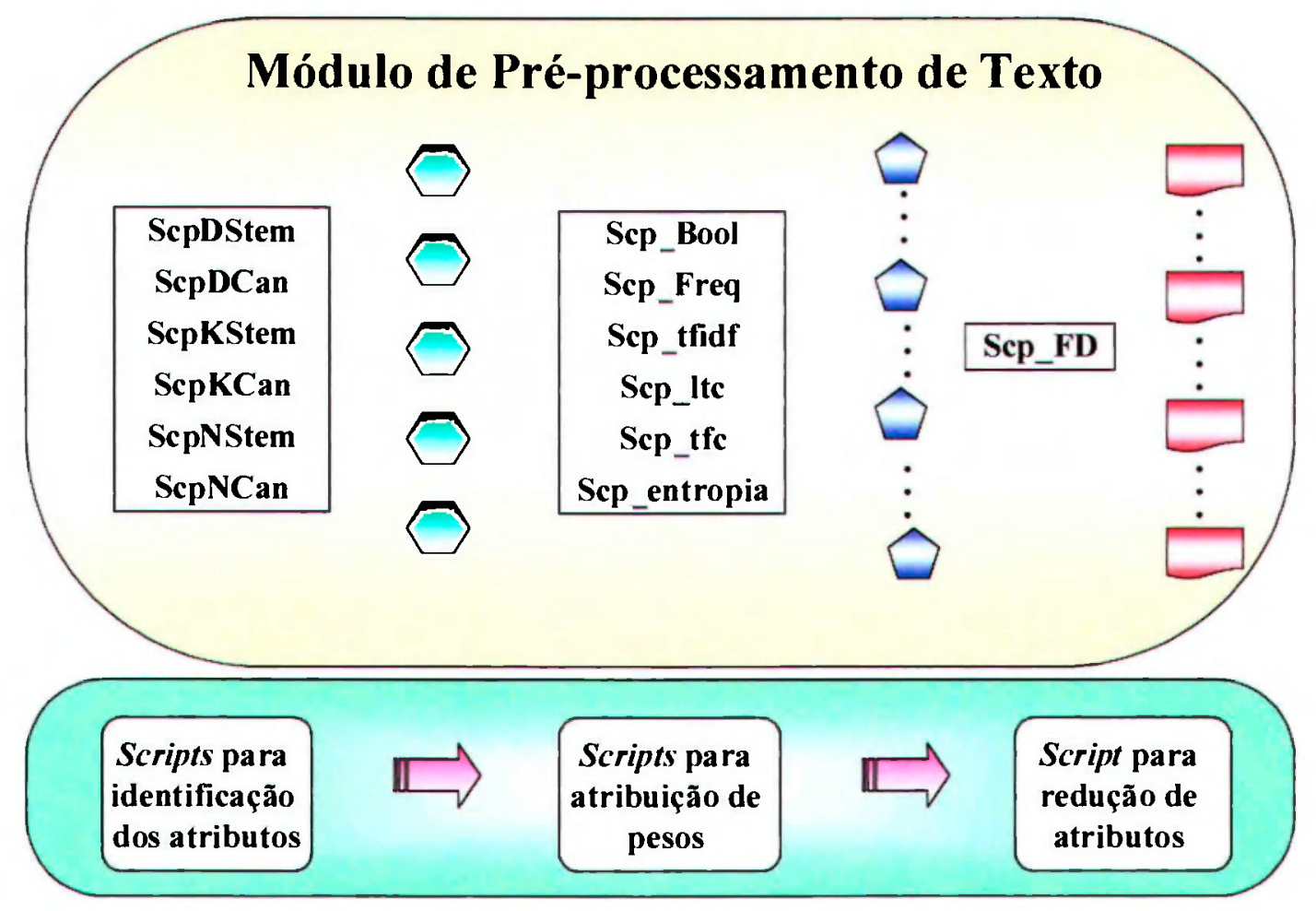

Figura 5.1: Scripts que integram o módulo de Pré-processamento de textos desenvolvidos em (Imamura 2001)

custoso, principalmente considerando textos de grande dimensão. Neste script os grams são normalizados, utilizando-se da identificação na forma canônica para representação.

O script ScpDStem diferencia do script ScpDCan apenas por normalizar os grams através do programa de stemming.

O script ScpNCan foi implementado com base nas definições de Pré-processamento apresentadas em (Weiss \& Indurkhya 1998), identificando atributos formados por apenas uma palavra (1-gram) e utilizando-se da identificação na forma canônica para a normalização. O script. ScpNCan, encontra os atributos preliminares, os $N$ termos mais freqüientes. Após essa primeira representação, são removidas as stopwords e identificados os termos mais relevantes.

O script ScpNStem diferencia do script. ScpNCan apenas por normalizar cada termo utilizando o algoritmo de stemming.

Os scripts ScpKStem e ScpKCan foram desenvolvidos baseados no algoritmo 
utilizado na ferramenta Kea (Witten, Paynter, Frank, Gutwin, \& Nevill-Manning 1999), sendo que o primeiro faz uso do programa de identificação baseado stemming e o segundo faz uso do etiquetador para a identificação na forma canônica.

Os scripts da ferramenta Kea foram logo descartados neste trabalho, uma vez que geravam muitos atributos por não retirarem as stopwords e ainda gerarem atributos $n$ grams, $\operatorname{com} n \leq 3$. No estudo apresentado em (Imamura 2001) foram gerados 56.886 atributos para o script ScpKStem e 77.271 atributos para o script ScpKCan. Com um conjunto de atributos tão grande, haveria necessidade de uma grande redução, principalmente para utilização dos algoritmos da ferramenta Weka, inviabilizando assim o uso dos scripts ScpKStem e ScpKCan.

Em Imamura (2001) também foram desenvolvidos scripts para atribuição de pesos, a fim de associar pesos aos atributos e obter a representação atributo-valor. No script Scp_Bool é atribuído aos atributos o peso booleano, indicando a presença ou ausência dos atributos. No script Scp_Freq o peso dos atributos corresponde à sua freqüência no documento. Nos scritps Scp_tfidf, Scp_tfc, Scp_ltc e Scp_entropia o peso associado aos atributos corresponde às medidas tfidf, tfc, ltc e entropia, respectivamente, descritos anteriormente na Seção 2.2.2.

Em Imamura (2001) também foi desenvolvido um script para redução do número de atributos, o script $\operatorname{Scp} F D$, que reduz a representação aos $N$ atributos mais representativos, com base na freqüência.

Somente alguns dos scripts desenvolvidos em (Imamura 2001) são utilizados nos experimentos descritos a seguir, uma vez que este trabalho está focado na extração de conhecimento.

\subsection{Planejamento dos Experimentos}

Os objetivos dos experimentos são a análise e comparação dos algoritmos de categorização de textos para extração de conhecimento com apoio das técnicas de Pré-processamento, desenvolvidas em (Imamura 2001) e utilizadas neste trabalho.

As etapas do processo de Text Mining foram descritas no Capítulo 2 e são agora detalhadas, enfocando os experimentos. Os experimentos, aqui realizados, seguem o 
esquema mostrado na Figura 5.2.

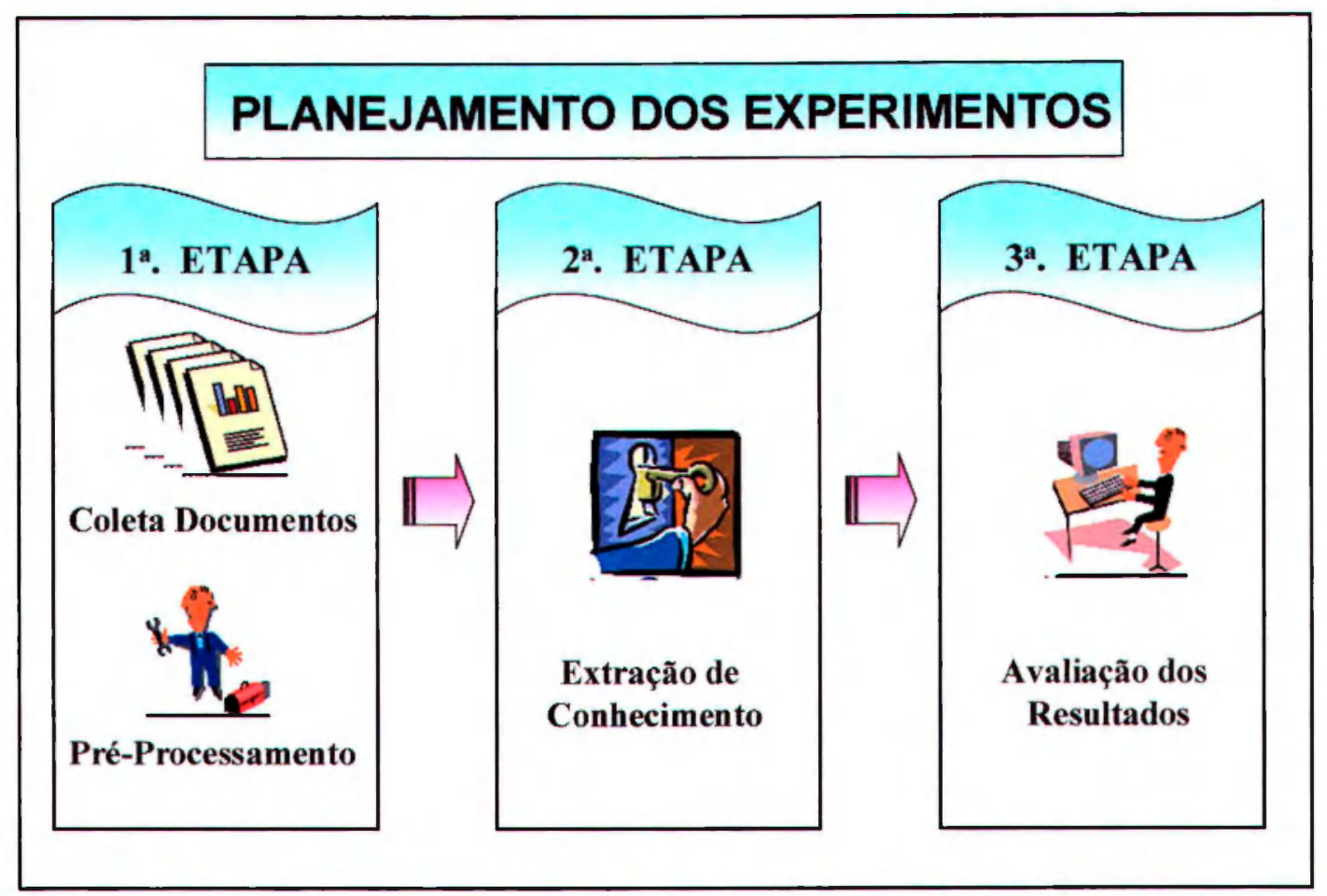

Figura 5.2: As etapas do processo de Text Mining no planejamento dos experimentos

Na Primeira Etapa, os documentos foram coletados e pré-processados utilizando diferentes técnicas desenvolvidas e relatadas em (Imamura 2001). Com a representação dos documentos no formato atributo-valor, na Segunda Etapa foram aplicados diferentes algoritmos de categorização de textos, e na Terceira Etapa foram avaliados os resultados obtidos.

\subsubsection{Primeira Etapa: Coleta de Dados e Pré-processamento}

Investir no processamento computacional da nossa língua é a única forma de garantir que a sociedade de informação do futuro privilegie a língua portuguesa para comunicar, ensinar e ter acesso ao conhecimento, permitindo que o cidadão comum possa viver com os computadores sem ter de deixar de lado sua cultura e sua língua. Para isto, é essencial que sejam desenvolvidos métodos de avaliação, teste e comparação, juntamente com coleções de documentos para testes, como ocorre nas conferências americanas TREC ( Text RE- 
trievel Conference $)^{2}$ e MUC (Message Understanding Conference) $)^{3}$, entre outras, mas específicos para o português.

Não foi encontrada nenhuma base de textos em português que pudesse servir como benchmark para ser utilizada como referência ou respaldo para utilização das técnicas abordadas neste trabalho. Em face disso, utilizou-se neste trabalho base textual com documentos sobre Aquisição de Conhecimento e Redes Neurais, coletada e utilizada em (Imamura 2001). Foram coletados manualmente 152 documentos da Web, sendo 76 rotulados como Aquisição de Conhecimento (AC), considerada como a classe positiva e 76 rotulados como Redes Neurais (RN), portanto tem-se um problema de categorização bi-valorada.

Os documentos coletados são dos mais variados formatos: HTML, Post Script, ASCII entre outros sendo todos transformados para arquivos ASCII, sem qualquer tag HTML, figuras, etc. Uma síntese do tamanho dos documentos ${ }^{4}$ pode ser observada na Tabela 5.1.

\begin{tabular}{|c||c|c|c|}
\hline $\begin{array}{c}\text { Total de } \\
\text { Documentos }\end{array}$ & $\begin{array}{c}\text { Número total } \\
\text { de palavras }\end{array}$ & $\begin{array}{c}\text { Número palavras } \\
\text { do maior documento }\end{array}$ & $\begin{array}{c}\text { Número palavras } \\
\text { do menor documento }\end{array}$ \\
\hline \hline $76(\mathrm{AC})($ Classe +$)$ & 10.366 & 1.767 & 66 \\
\hline $76(\mathrm{RN})$ & 10.528 & 3.179 & 76 \\
\hline \hline
\end{tabular}

Tabela 5.1: Tamanho dos documentos utilizados nos experimentos

As principais atividades realizadas no Pré-processamento até finalizar a identificação dos atributos são:

1. Case Folding: consiste em converter todos os caracteres do documento para um mesmo formato, ou seja, somente letras maiúsculas ou letras minúsculas. Neste trabalho, optou-se por transformar todos os documentos em letras minúsculas.

2. Stopwords: consiste em retirar palavras que aparecem em todos os documentos, e portanto não diferenciam os documentos entre si. As stopwords, podem ser

\footnotetext{
${ }^{2}$ http://trec.nist.gov/

${ }^{3} \mathrm{http} / /$ www.ldc.upenn.edu/Catalog/MUC.html

${ }^{4}$ Os documentos estão disponiveis em http://labic.icmc.sc.usp.br
} 
preposições, artigos, pronomes, etc. Neste trabalho, a remoção das stopwords é feita verificando a presença em uma lista de 613 palavras, criada por Imamura (2001).

3. Stemming: consiste em converter cada palavra a sua forma radical, ou seja, sua forma neutra sem inflexões verbais, de gênero ou de número. Consiste em agrupar palavras de mesma raiz léxica/etimológica com o objetivo de reunir em um único termo as palavras de mesma raiz semântica. Trata-se de trabalhar com uma espécie de máximo divisor comum das palavras, ou tronco representativo de cada uma destas palavras do grupo (eliminando diferenças léxicas irrelevantes tais como plural, tempo verbal, sufixos e prefixos, etc.). Todas estas idéias foram vigorosamente investigadas nos anos sessenta e setenta e persistem na maioria dos sistemas usados hoje, tanto no ambiente acadêmico quanto no comercial. Neste trabalho é utilizado o algoritmo de stemming baseado no algoritmo de Porter (1980), que é um dos algoritmos mais tradicionais e disseminados entre os pesquisadores de Text Mining.

4. Identificação de Formas Canônicas: consiste em normalizar palavras baseandose na análise morfológica da palavra e na análise gramatical da sentença em que se encontra. Para encontrar essa forma canônica, contou-se com um etiquetador, uma ferramenta desenvolvida no NILC (Nunes 1996).

As atividades 1 e 2 necessariamente são realizadas para identificação dos atributos, já as atividades 3 e 4 são alternativas para normalização de atributos, conforme ilustrado na Figura 5.3.

A fim de exemplificar as diferentes abordagens utilizadas para normalização das palavras utilizadas para identificação de atributos é considerado o seguinte trecho ${ }^{5}$.

"Busca por informação deve congestionar sites. No dia da eleição de 1996, milhões de pessoas acessaram o ciberespaço pela primeira vez para buscar informaçôes de último minuto sobre a corrida presidencial."

Em relação ao texto descrito anteriormente, fazendo-se uso do stemming baseado no algoritmo de Porter, e também o uso do Etiquetador para identificação da forma canônica, na Tabela 5.2 é mostrado o resultado desses dois algoritmos para algumas palavras.

\footnotetext{
${ }^{5}$ Extraído da página http://www.estado.com.br/editoriais/2000/11/05/int867.html
} 


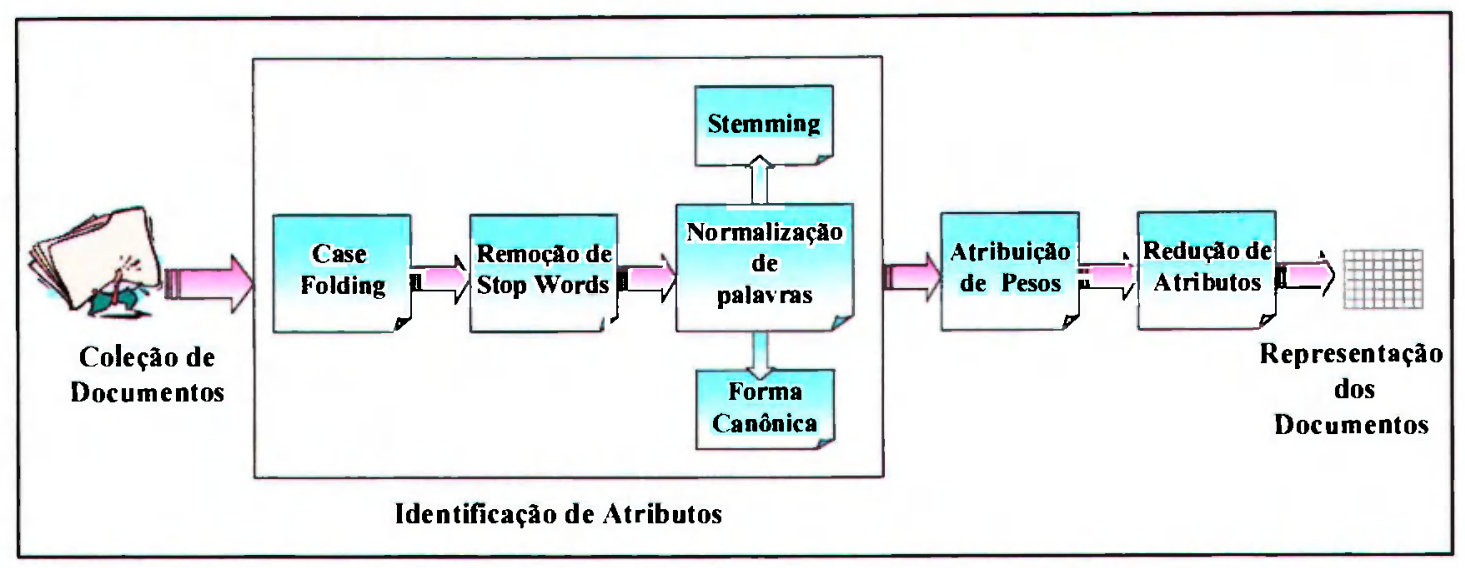

Figura 5.3: Principais atividades do Pré-processamento

\begin{tabular}{|c||c|}
\hline \hline $\begin{array}{c}\text { Identificação da Forma Canônica } \\
\text { (uso do Etiquetador) }\end{array}$ & $\begin{array}{c}\text { Stemming } \\
\text { (algoritmo de Porter) }\end{array}$ \\
\hline de $\rightarrow$ PREPOSIÇÃO & de $\rightarrow$ de \\
\hline busca $\rightarrow$ VERBO[buscar] & busca $\rightarrow$ busc \\
\hline informação $\rightarrow$ SUBSTANTIVO[informação] & informação $\rightarrow$ inform \\
\hline informações $\rightarrow$ SUBSTANTIVO[informação] & informações $\rightarrow$ inform \\
\hline pessoas $\rightarrow$ SUBSTANTIVO[pessoa] & pessoas $\rightarrow$ pesso \\
\hline minuto $\rightarrow$ SUBSTANTIVO[minuto] & minuto $\rightarrow$ minut \\
\hline presidencial $\rightarrow$ ADJETIVO[presidencial] & presidencial $\rightarrow$ presidencial \\
\hline \hline
\end{tabular}

Tabela 5.2: Exemplo da saída do algoritmo usando o etiquetador para identificação da forma canônica e stemming

Na Figura 5.4 são apresentados os scripts utilizados neste trabalho para o Préprocessamento dos textos.

Primeiramente para a identificação dos atributos, representando os documentos no formato atributo-valor, utilizou-se quatro scripts: ScpDCan, ScpDStem, ScpNCan e ScpNStem. Após a identificação dos atributos, gerados pelos scripts mencionados tern-se o número total de atributos.

Para finalizar a etapa de Pré-processamento e encontrar a representação final dos documentos, ainda é necessário realizar as atividades de atribuição de pesos e seleção de atributos, conforme ilustrado na Figura 5.3 


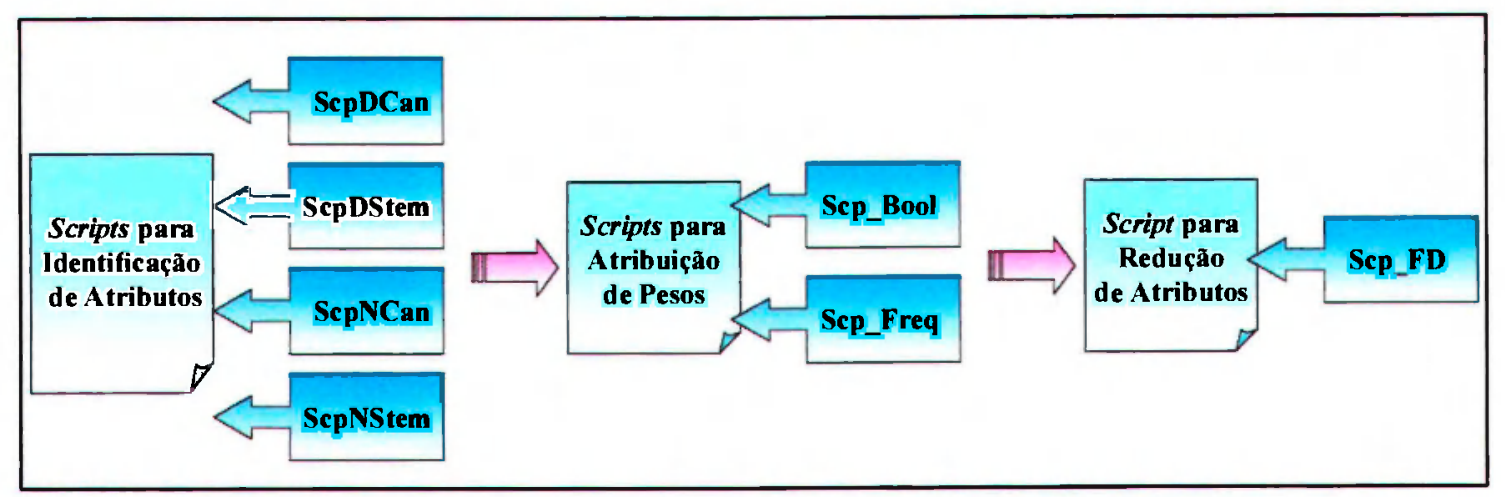

Figura 5.4: Scripts que integram o módulo de Pré-processamento e utilizados neste trabalho

Como já citado, o peso de um atributo pode ser determinado segundo algumas abordagens, que são baseadas em duas informações empíricas em relação a textos: a quantidade de vezes que o termo aparece em um documento e a quantidade de vezes que o termo é encontrado em todos os documentos da coleção. Neste trabalho foi utilizada somente a primeira abordagem.

Baseado nos resultados obtidos em (Imamura 2001), verificou-se que as representações mais simples, booleana e freqüência, foram as que obtiveram melhores resultados. Por estes motivos foram utilizados os scripts Scp_Bool e Scp_Freq, respectivamente, para peso booleano e freqüência para o cálculo dos pesos dos atributos a serem utilizados neste trabalho.

A atribuição de pesos utilizadas nos experimentos neste trabalho, como já ilustradas na Figura 5.4, são:

- Booleana - o peso designa a presença ou ausência de um termo em um documento.

Caso exista, o atributo assume o valor 1 , caso contrário, assume o valor 0 .

$a_{i k}= \begin{cases}1, & \text { se } f_{i k}>0 \\ 0, & \text { caso contrário }\end{cases}$

- Freqüência - o peso designa a quantidade de vezes que um termo aparece em um documento. Esta medida parte do princípio que termos raros não são muito informativos para classificação. 


$$
a_{i k}=f_{i k}
$$

Uma visão dos dataset a serem utilizados nos experimentos pode ser observada na Tabela 5.3. Os conjuntos DCan_set e DStem_set possuem um número maior de atributos pelo fato de usar 5-grams para construção dos atributos, enquanto NStem_set e NCan_set usam apenas 1-gram. Ressalta-se também que ao se utilizarem 1-gram, o número de palavras e atributos é igual, pois todos os atributos são compostos por apenas uma palavra.

\begin{tabular}{|c||c|c|c|}
\hline \hline Representação Dados & $\begin{array}{c}\text { Número } \\
\text { Palavras }\end{array}$ & $\begin{array}{c}\text { Número } \\
\text { Caracteres }\end{array}$ & $\begin{array}{c}\text { Número } \\
\text { atributos }\end{array}$ \\
\hline \hline DCan_set & 39.220 & 303.548 & 13.849 \\
\hline DStem_set & 39.990 & 255.824 & 15.854 \\
\hline NCan_set & 2.068 & 16.750 & 2.068 \\
\hline NStem_set & 1.956 & 12.149 & 1.956 \\
\hline \hline
\end{tabular}

Tabela 5.3: Informações sobre os arquivos gerados pelos scripts de identificação de atributos

Além da identificação dos atributos e de atribuição de pesos, um dos grandes problemas encontrados na realização da extração de conhecimento foi a construção da representação dos dados no formato atributo-valor. A forma como os arquivos de dados são especificados para cada ferramenta utilizada na extração de padrões, também influencia algumas soluções adotadas. Tais formatos, que acabam gerando um grande volume de dados, acabam influenciando em muito o desempenho das ferramentas, impossibilitando muitas vezes até a execução destas.

Esse problema ocorre devido a algumas propriedades peculiares da representação de documentos como um vetor hiperdimensional de atributos. Ao contrário da maioria das bases de dados convencionais, em que o número de atributos é irrelevante comparando-se ao número de exemplos, quando se realiza Text Mining o grande número de atributos pode limitar a escolha de algumas ferramentas.

A notação vetorial de documentos possui uma quantidade de atributos relevante (no caso em questão, palavras pertencentes ao documento), chegando a dezenas de milhares de atributos disponíveis para categorização dos textos. Outra diferença em relação 
as base de dados convencionais, é que os vetores de documentos são geralmente muito esparsos, isto é, a maioria de suas coordenadas possuem valores nulos.

Por motivo de tratabilidade computacional, diversas técnicas têm sido estudadas para reduzir o tamanho da representação dos documentos. Além do Pré-processamento dos dados, em que já há uma redução com a eliminação de stopwords, redução pela aplicação do algoritmo de stemming, ou pela extração da forma canônica, há ainda a necessidade de se aplicar algoritmos de redução de atributos.

Para auxiliar na redução das representações de alta dimensão para que elas não excedam a capacidade de processamento de muitos dos algoritmos utilizados para extração de conhecimento, há várias técnicas ou métodos para se fazer a redução de features, como: Freqüência de Documento (FD), Ganho de Informação(GI), Informação Mútua (IG) e $\chi^{2}$ Estatístico (CHI), conforme já visto no Capítulo 2.

Nos experimentos a serem realizados, é utilizado para redução de features o scriptScp_FD, como ilustrado na Figura 5.4. Para esse script é necessário informar qual é a quantidade de features a ser selecionada. Para cada redução desejada, o arquivo com as features é ordenado por ordem decrescente de valor sendo selecionado somente as $N$ primeiras features, onde $N$ é o limite de seleção. As palavras mais comuns, stopwords não são consideradas nesse método já que elas foram eliminadas anteriormente. Nos experimentos a serem realizados o $N$ é: 250,500,750,1.000, 1.500 para os conjuntos NStem_set e NCan_set. Para os conjuntos DCan_set e DStem_set o $N$ ficou em: $250,500,750,1.000,1.500$ e 3.000. Como pode ser observado na Tabela 5.3, os conjuntos de dados NStem_set e NCan_set não atingem 3.000 atributos, portanto não podem ser reduzidos a esse valor.

A vantagem de se utilizar Frequêencia de Documento (FD) como método de redução de features é que esta é uma das técnicas mais simples além de facilmente escalonável para grandes conjuntos de textos por possuir uma complexidade computacional aproximadamente linear em relação aos números de documentos. Na Tabela 5.4 são apresentados os resultados obtidos pela aplicação das reduçōes.

$\mathrm{Na}$ literatura, para língua portuguesa não há justificativas para saber até quantas n-grams seriam necessárias para a utilização do algoritmo sugerido em (Mladenić 1998). 
Portanto utilizou-se 5-grams conforme sugerido em (Mladenic 1998) para o caso da língua inglesa.

Dando continuidade ao trabalho desenvolvido em (Imamura 2001), continuou-se a utilizar o 5-grams para o dataset DCan_set e DStem_set. Observa-se que a medida que se reduz o número de features ao utilizar o algoritmo de redução de features, as features resultantes podem não ser compostas pelos 5-grams, como pode ser observado na Tabela 5.5. Esta tabela demonstra a distribuição dos $n$-grams, de 1 a 5 grams, sobre o arquivo utilizado nos experimentos. Pode-se observar que com a redução necessária para poder utilizar-se da ferramenta Weka a percentagem de 5-grams é extremamente baixa.

Nos experimentos, para o português, não há necessidade de se utilizar 5-grams, conforme pode ser observado pelos dados mostrados na Tabela 5.5. Com o dataset DCan_set e tomando-se como referência 1-gram e 2-grams, na redução para 250 features tem-se $100 \%$ do total dos 250 atributos. Na redução para 500 features tem-se $99,40 \%$ do total dos 500 atributos. Na redução para 750 features tem-se $98,53 \%$ do total dos 750 atributos. $\mathrm{Na}$ redução para 1.000 features tem-se $93,60 \%$ do total dos 1.000 atributos. Na redução para 1.500 features tem-se $87,47 \%$ do total dos 1.500 atributos. Na redução para 3.000 features tem-se $68,20 \%$ do total dos 3.000 atributos.

Com o dataset DStem_set e tomando-se como referência 1-gram e 2-grams, na redução para 250 features tem-se $98,80 \%$ do total dos 250 atributos. Na redução para 500 features tem-se $99,90 \%$ do total dos 500 atributos. Na redução para 750 features tem-se $99,20 \%$ do total dos 750 atributos. Na redução para 1.000 features tem-se $99,40 \%$ do total dos 1.000 atributos. Na redução para 1.500 features tem-se $96,13 \%$ do total dos 1.500 atributos. Na redução para 3.000 features tem-se $83,07 \%$ do total dos 3.000 atributos.

Portanto, para a representação dos dados, aplicando-se redução de até 3.000 features poderia ter utilizado somente a representação até 2-grams e não 5-grams como foi utilizado.

Após os passos de Pré-processamento tem-se o conjunto de dados pronto para ser processado. Uma síntese do planejamento dessa primeira etapa é mostrada na Figura 5.5, em que são ilustradas as representações intermediárias obtidas em cada atividade do Pré-processamento. 


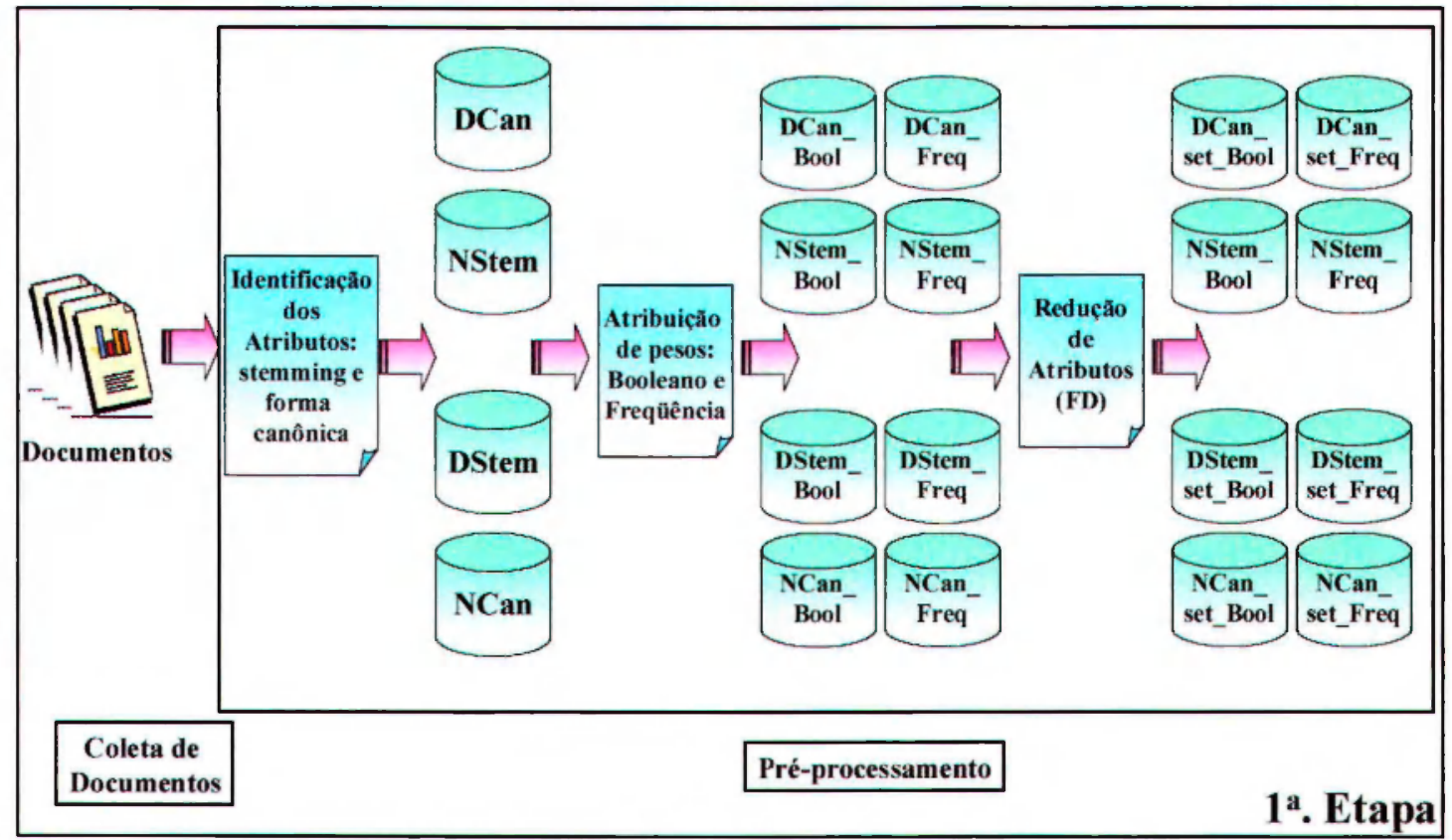

Figura 5.5: Coleta e Pré-processamento de documentos 


\begin{tabular}{|c|c|c|c|c|}
\hline Dataset & $\begin{array}{c}\text { Representaçāo dos Dados } \\
\text { Redução de features }\end{array}$ & $\begin{array}{l}\text { Número } \\
\text { Palavras }\end{array}$ & $\begin{array}{l}\text { Número } \\
\text { Caracteres }\end{array}$ & $\begin{array}{l}\text { Número } \\
\text { Atributos }\end{array}$ \\
\hline \multirow{6}{*}{ DCan_set } & 3.000 & 6.652 & 54.952 & 3.000 \\
\hline & 1.500 & 2.400 & 19.505 & 1.500 \\
\hline & 1.000 & 1.328 & 10.631 & 1.000 \\
\hline & 750 & 866 & 6.886 & 750 \\
\hline & 500 & 553 & 4.292 & 500 \\
\hline & 250 & 266 & 2.073 & 250 \\
\hline \multirow{6}{*}{ DStem_set } & 3.000 & 5.257 & 32.423 & 3.000 \\
\hline & 1.500 & 1.978 & 11.763 & 1.500 \\
\hline & 1.000 & 1.171 & 6.933 & 1.000 \\
\hline & 750 & 844 & 4.916 & 750 \\
\hline & 500 & 535 & 3.053 & 500 \\
\hline & 250 & 260 & 1.465 & 250 \\
\hline \multirow{5}{*}{ NCan_set } & 1.500 & 1.500 & 12.378 & 1.500 \\
\hline & 1.000 & 1000 & 8.062 & 1.000 \\
\hline & 750 & 750 & 5.973 & 750 \\
\hline & 500 & 500 & 3.880 & 500 \\
\hline & 250 & 250 & 1.933 & 250 \\
\hline \multirow{5}{*}{ NStem_set } & 1.500 & 1.500 & 9.232 & 1.500 \\
\hline & 1.000 & 1000 & 5.991 & 1.000 \\
\hline & 750 & 750 & 4.460 & 750 \\
\hline & 500 & 500 & 2.883 & 500 \\
\hline & 250 & 250 & 1.379 & 250 \\
\hline
\end{tabular}

Tabela 5.4: Informaçōes sobre os arquivos gerados pelos scripts de identificação de atributos, após as reduções realizadas 


\begin{tabular}{|c|c|c|c|c|c|c|}
\hline Dataset & $\begin{array}{c}\text { Representação dos Dados } \\
\text { Redução }\end{array}$ & 1-gram & 2 -grams & 3-grams & 4-grams & 5-grams \\
\hline & 250 features & $\begin{array}{c}234 \\
(93,60 \%) \\
\end{array}$ & $\begin{array}{c}16 \\
(6,40 \%)\end{array}$ & 0 & 0 & 0 \\
\hline & 500 features & $\begin{array}{c}450 \\
(90,00 \%) \\
\end{array}$ & $\begin{array}{c}47 \\
(9,40 \%)\end{array}$ & $\begin{array}{c}3 \\
(0,60 \%)\end{array}$ & 0 & 0 \\
\hline \multirow[t]{6}{*}{ DCan_set } & 750 features & $\begin{array}{c}645 \\
(86,00 \%) \\
\end{array}$ & $\begin{array}{c}94 \\
(12,53 \%)\end{array}$ & $\begin{array}{c}11 \\
(1,47 \%) \\
\end{array}$ & 0 & 0 \\
\hline & 1.000 features & $\begin{array}{c}769 \\
(76,90 \%) \\
\end{array}$ & $\begin{array}{c}167 \\
(16,70 \%) \\
\end{array}$ & $\begin{array}{c}42 \\
(4,20 \%) \\
\end{array}$ & $\begin{array}{c}11 \\
(1,10 \%) \\
\end{array}$ & $\begin{array}{c}11 \\
(1,10 \%) \\
\end{array}$ \\
\hline & 1.500 features & $\begin{array}{c}880 \\
(58,67 \%) \\
\end{array}$ & $\begin{array}{c}432 \\
(28,80 \%) \\
\end{array}$ & $\begin{array}{c}110 \\
(7,33 \%) \\
\end{array}$ & $\begin{array}{c}64 \\
(4,27 \%) \\
\end{array}$ & $\begin{array}{c}14 \\
(0,93 \%) \\
\end{array}$ \\
\hline & 3.000 features & $\begin{array}{c}1.064 \\
(35,47 \%) \\
\end{array}$ & $\begin{array}{c}982 \\
(32,73 \%) \\
\end{array}$ & $\begin{array}{c}424 \\
(14,13 \%) \\
\end{array}$ & $\begin{array}{c}298 \\
(9,93 \%) \\
\end{array}$ & $\begin{array}{c}232 \\
(7,73 \%) \\
\end{array}$ \\
\hline & 250 features & $\begin{array}{c}243 \\
(97,20 \%)\end{array}$ & $\begin{array}{c}4 \\
(1,60 \%)\end{array}$ & $\begin{array}{c}3 \\
(1,20 \%)\end{array}$ & 0 & 0 \\
\hline & 500 features & $\begin{array}{c}467 \\
(93,70 \%) \\
\end{array}$ & $\begin{array}{c}31 \\
(6,20 \%)\end{array}$ & $\begin{array}{c}2 \\
(0,40 \%) \\
\end{array}$ & 0 & 0 \\
\hline \multirow[t]{4}{*}{ DStem_set } & 750 features & $\begin{array}{c}662 \\
(88,27 \%) \\
\end{array}$ & $\begin{array}{c}82 \\
(10,93 \%) \\
\end{array}$ & $\begin{array}{c}6 \\
(0,80 \%) \\
\end{array}$ & 0 & o \\
\hline & 1.000 features & $\begin{array}{c}835 \\
(83,50 \%)\end{array}$ & $\begin{array}{c}159 \\
(15,90 \%)\end{array}$ & $\begin{array}{c}6 \\
(0,60 \%) \\
\end{array}$ & $\begin{array}{c}0 \\
(\%)\end{array}$ & $\begin{array}{c}0 \\
(\%)\end{array}$ \\
\hline & 1.500 features & $\begin{array}{c}1.104 \\
(73,60 \%) \\
\end{array}$ & $\begin{array}{c}338 \\
(22,53 \%) \\
\end{array}$ & $\begin{array}{c}39 \\
(2,60 \%) \\
\end{array}$ & $\begin{array}{c}14 \\
(0,94 \%) \\
\end{array}$ & $\begin{array}{c}5 \\
(0,33 \%) \\
\end{array}$ \\
\hline & 3.000 features & $\begin{array}{c}1.539 \\
(51,30 \%)\end{array}$ & $\begin{array}{c}953 \\
(31,77 \%)\end{array}$ & $\begin{array}{c}297 \\
(9,90 \%)\end{array}$ & $\begin{array}{c}134 \\
(4,47 \%)\end{array}$ & $\begin{array}{c}77 \\
(2,56 \%)\end{array}$ \\
\hline
\end{tabular}

Tabela 5.5: Informações sobre os arquivos gerados pelos scripts com relação aos $n$-grams 


\subsubsection{Segunda Etapa: Extração de Conhecimento}

Nos experimentos realizados foi utilizado o 10-fold cross-validation, onde todo o conjunto de exemplos é dividido em 10 partições diferentes, aleatórias e mutuamente exclusivas. São realizadas 10 iteraçōes e em cada iteração, uma partição diferente é utilizada para testar e as outras 9 partições restantes são utilizadas para treinar o sistema. A taxa de erro é resultado das médias das taxas de erro das 10 iteraçōes. Uma das vantagens de utilização dos métodos de cross-validation é que todos os exemplos são usados para testar e todos são usados para treinar o sistema.

Na extração de padrões realizada neste trabalho, seis diferentes algoritmos disponíveis nas ferramentas $S V M^{\text {light }}$ e Weka foram utilizados:

- SVM - indutor implementado no $S V M^{\text {light }}$;

- Naive Bayes - indutor implementado no Weka;

- Árvore de Decisão - indutor de Árvore de Decisão alternante implementado no Weka, que gera Árvores de Decisão otimizadas para problemas de classificação binários;

- Regras de Decisão - algoritmo OneR pertencente ao Weka ;

- Tabela de Decisão - indutor implementado no Weka ;

- Vizinho mais Próximo - algoritmo implementado no Weka;

A escolha do SVM deve-se ao fato deste ser um dos mais citados na literatura para categorizaçāo de textos, conforme visto no Capítulo 4 onde o SVM foi detalhado, sendo feito também referência a vários autores que já fizeram comparações de algoritmos de categorização para textos em inglês. A ferramenta Weka disponibiliza a implementação dos algoritmos citados acima e trabalha com a notação convencional para representar documentos, estando disponível no LABIC.

Para utilizar as ferramentas $S V M^{\text {light }}$ e Weka foram implementados dois scripts em linguagem Perl para converter os conjuntos de dados para os formatos de entrada das ferramentas. Um esquema geral representando os conjuntos de dados e os algoritmos é mostrado na Figura 5.6. 


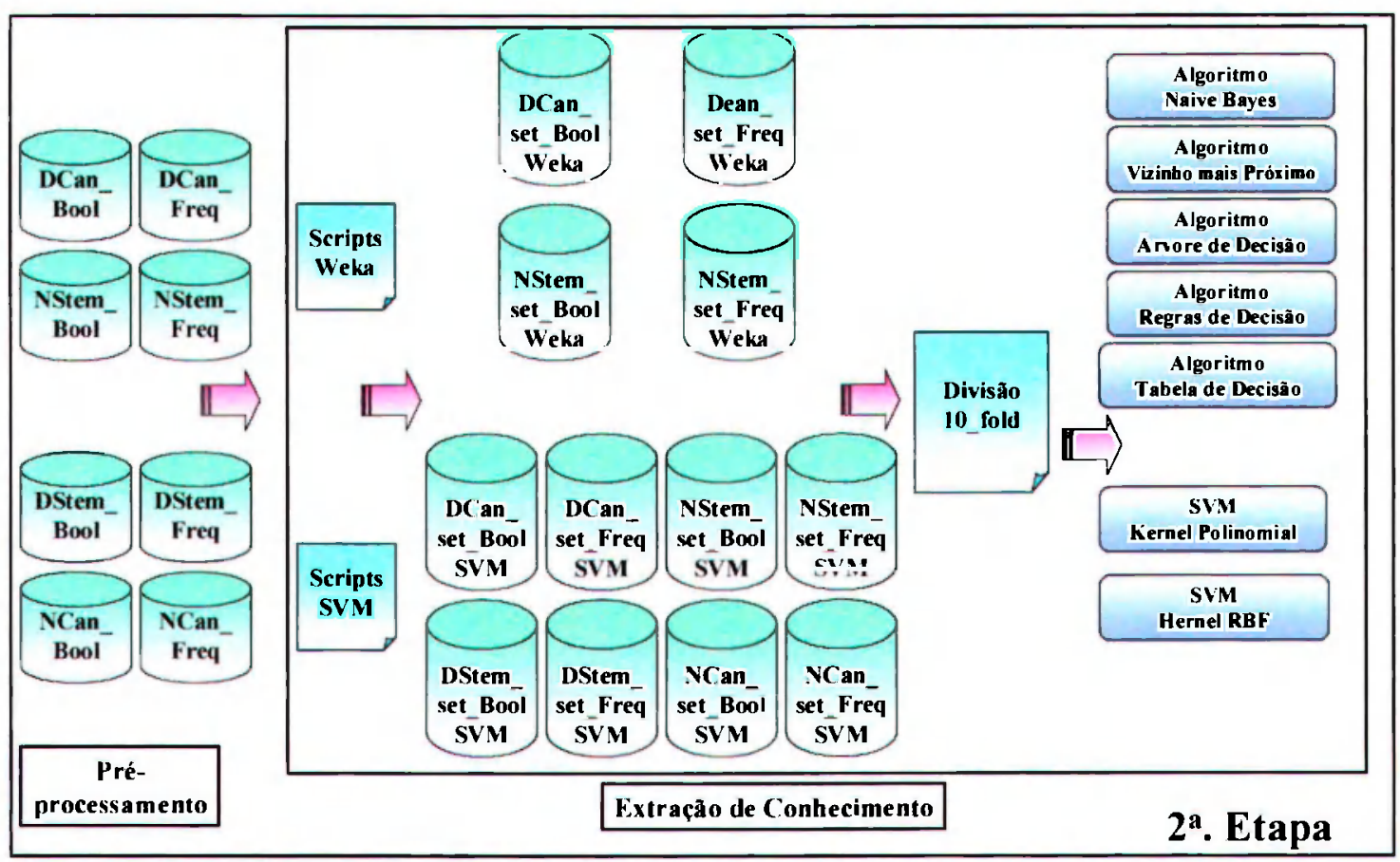

Figura 5.6: Segunda etapa: extração de conhecimento

Na Figura 5.7 pode ser observado o esquema que foi seguido nos experimentos para a aplicação do algoritmo SVM. Com este algoritmo foram realizados experimentos utilizando configurações com as funções Kernel polinomial e Radial Basis, com seus respectivos parâmetros, $\mathbf{d}$ e g e cujas equações são:

- Função polinomial $(\mathrm{t}=1):(s a * b+c)^{d}$

- Função Radial Basis(RBF) $(\mathrm{t}=2):\left(-g\|a-b\|^{2}\right)$

Conforme visto no Capítulo 4, pode-se utilizar funções Kernels para tratamento de categorização de textos, quando os dados não são linearmente separáveis. Mesmo sabendo que no estudo de caso realizado tem-se dados linearmente separáveis, podese utilizar os modelos não lineares. Joachims (1998) explorou duas classes de SVMs não lineares: polinomial e RBF, e observou pequenos benefícios, comparados a modelos lineares. $S V M^{\text {light }}$ tem como parâmetros default kernel polinomial linear com parâmetros $\mathrm{t}=1$ e $\mathrm{d}=1$. 


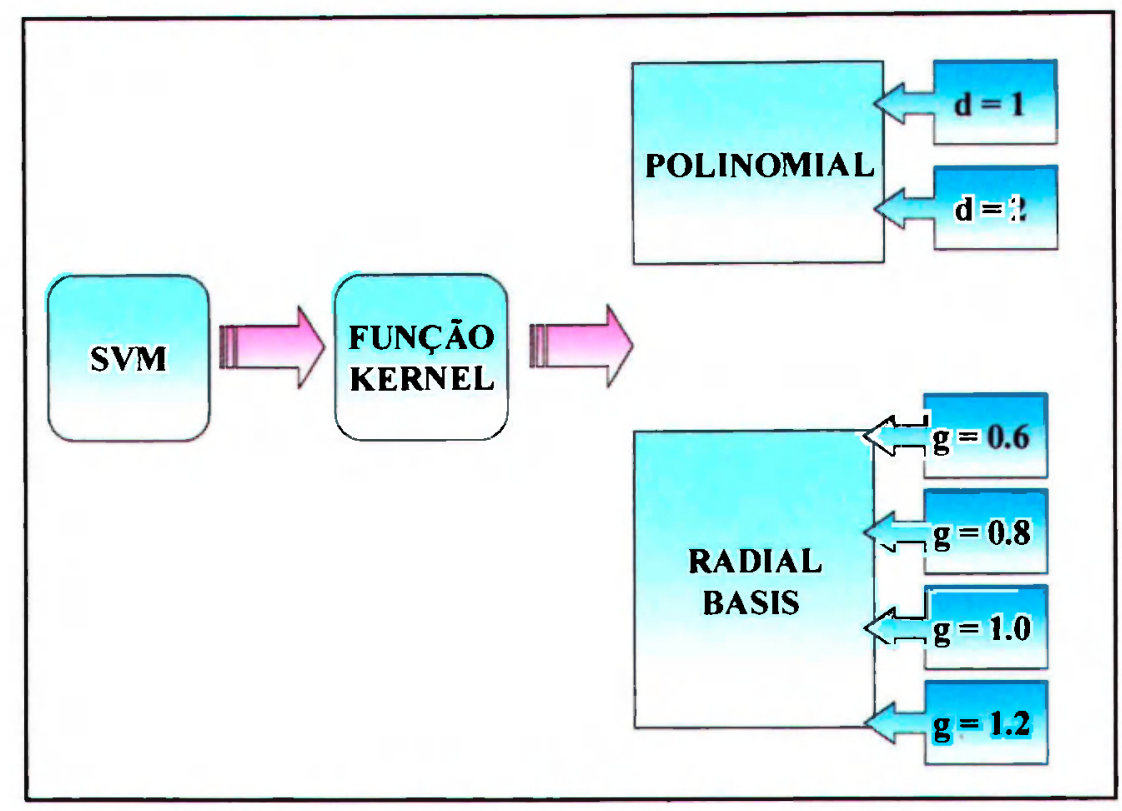

Figura 5.7: Esquema para o algoritmo SVM utilizando diferentes funções Kernel

Vale ressaltar que o $S V M^{\text {light }}$ é uma ferramenta que trabalha com a representação de vetores esparsos. Ao se utilizar esse formato de arquivo, as representações de vetores de documentos ocupam um espaço bem menor que a maneira convencional, às custas de um overhead gerado pela especificação do índice de atributo para cada valor não nulo. Como esse overhead é inversamente proporcional à quantidade de valores nulos, o custo gerado pelo uso dos índices é irrelevante, compensando o uso dessa notação. Somando-se a isso, o fato do $S V M^{\text {light }}$ efetuar todos os cálculos diretamente com vetores esparsos e o bom desempenho do algoritmo SVM, o $S V M^{\text {light }}$ obtém bons resultados para Text Mining.

Por outro lado, a ferramenta Weka trabalha com a notação convencional para representação de exemplos: valores de atributos separados por delimitadores. Apesar dessa notação possuir um overhead menor que a representação por vetores esparsos quando representando vetores com poucos valores de atributos nulos, a situação se inverte quando se realiza Text Mining pois normalmente existe muitos valores de atributos nulos.

Para facilitar a análise dos resultados. os experimentos realizados com a identificação de atributos na forma canônica, utilizando o etiquetador e o algoritmo baseado em (Mladenić 1998) (conjunto de dados DCan), foram denominados de Experimento 1. 
Os experimentos realizados com a identificação de atributos pelo método de stemming, utilizando as definições de Pré-processamento baseado em (Mladenić 1998) (conjunto de dados DStem), foram denominados de Experimento 2. Os experimentos realizados com a identificação de atributos na forma canônica, utilizando o etiquetador e utilizando as definições de Pré-processamento baseado em (Weiss \& Indurkhya 1998) (conjunto de dados NCan), foram denominados de Experimento 3. Os experimentos realizados com a identificação de atributos pelo método de stemming, utilizando as definições de Pré-processamento baseado em (Weiss \& Indurkhya 1998) (conjunto de dados NStem), foram denominados de Experimento 4.

Primeiramente foi utilizado o conjunto de dados, sem redução de atributos, para os quatro experimentos. Neste caso só foi possível a utilização do algoritmo de SVM, uma vez que esse algoritmo trabalha com vetores esparsos e assim não há necessidade de redução de atributos.

Para possibilitar o uso da ferramenta Weka e a utilização de todos os outros algoritmos, foi necessário realizar uma redução de features. A redução neste caso foi de 500 features e só foi aplicada para o Experimento 1 e Experimento 4. Com essa redução para os 2 conjuntos de dados DCan_set e NStem_set, foi possível utilizar as duas ferramentas, $S V M^{\text {light }}$ e $W e k a$, executando todos os algoritmos propostos. Não foi possível utilizar reduções maiores pelas limitações da ferramenta Weka.

Com a finalidade de se analisar os efeitos da redução de features sobre os resultados alcançados, em seguida fez-se a redução para $250,750,1.000,1.500$ e 3.000 features. A redução de features para 3.000 só é possível aplicar nos Experimento 1 e Experimento 2, em função desses experimentos possuir um número de atributos superior a 3.000 , como pode ser observado na Tabela 5.3. Nesses casos, foi possível a utilização somente da ferramenta $S V M^{\text {light }}$ para os quatro experimentos.

Após esta etapa de Extração de Conhecimento tem-se a terceira etapa onde se irá calcular a estimativa da taxa de erro dos classificadores, para a categorização sob o conjunto inteiro através da média e desvio padrão das medidas de desempenho dos modelos parciais. 


\subsubsection{Terceira Etapa: Avaliação dos Resultados}

Para validar o conhecimento extraído, é necessário utilizar-se de métricas para avaliar o desempenho do classificador gerado. Neste trabalho, as medidas utilizadas para a avaliação de desempenho, são ilustradas na Figura 5.8 e são as seguintes: erro total, erro da classe positiva, cobertura e $F_{1}$, conforme visto no Capítulo 2 .

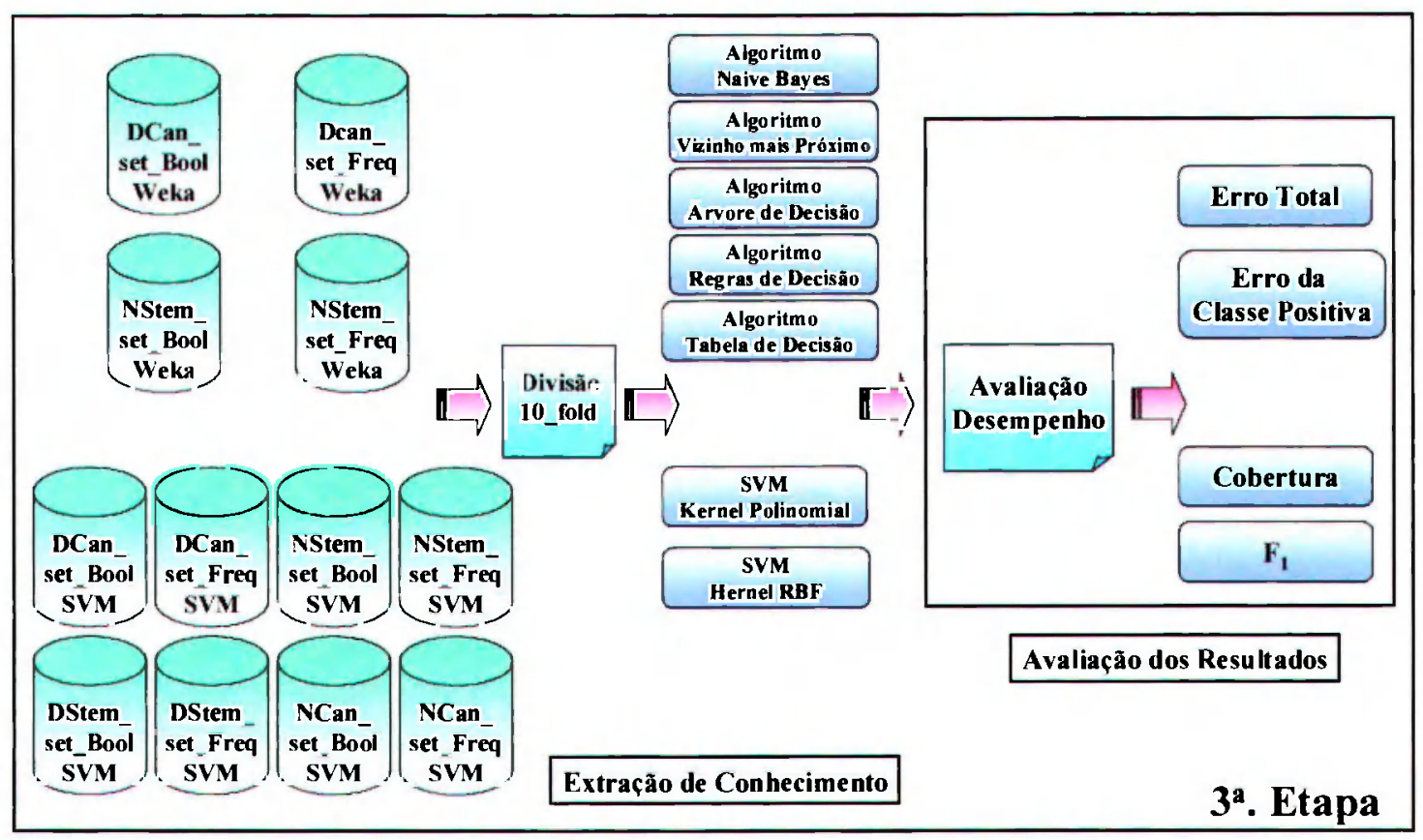

Figura 5.8: Terceira etapa: avaliação dos resultados

Os dados da avaliação de desempenho foram importados para o Microsoft Excel 97 e para cada uma dessas medidas, foram calculados as médias e os respectivos desviospadrão.

\subsection{Considerações Finais}

Utilizando-se os scripts ScpDCan, ScpDStem. ScpNCan, ScpNStem, Scp_Bool, Scp_Freq, Scp_FD, os scripts para conversão e as ferramentas $S V M^{\text {light }}$ e Weka, descritos nas seções anteriores, e seguindo as diretrizes especificadas do Planejamento dos Experimentos, no próximo capítulo são mostrados os resultados obtidos. 


\section{Capítulo 6}

\section{Apresentação dos Resultados}

\subsection{Considerações Iniciais}

As medidas de desempenho utilizadas na avaliação dos resultados, descritas no Capítulo 2 , são erro total, erro da classe positiva, cobertura e $F_{1}$, e estão indicadas para cada experimento realizado, através de suas respectivas médias e desvios padrões, calculados a partir dos resultados parciais de cada um dos segmentos da validação cruzada 10 fold.

Foram realizados experimentos utilizando diferentes formas de identificação de features e atribuições de pesos booleano e por freqüência. Para as reduções de features aplicou-se o script scp_FD, selecionando diferentes quantidades.

Com o algoritmo SVM, da ferramenta $S V M^{\text {light }}$, foram utilizados os conjuntos de dados DCan_set, DStem, NCan e NStem. Para esses conjuntos foram aplicadas as reduções: primeiramente sem redução de features, com redução para 250, com redução para 500, com redução para 750, com redução para 1.000, com redução para 1.500 e finalmente com redução para 3.000 features. Para os conjuntos NCan e NStem não foi possível fazer a redução de features para 3.000, uma vez que esses conjuntos não possuem 3.000 atributos.

Com a ferramenta Weka foram utilizados dois conjuntos de dados DCan_set e NStem com redução para 500 features, que foram as que apresentavam melhores resultados com o algoritmo SVM.

Para verificar o comportamento comparativo dos classificadores obtidos utilizando 
tanto a ferramenta $S V M^{\text {light }}$ como para Weka e considerando também as reduções de features são calculadas as diferenças em desvios-padrão como mostrado na Seção 2.2 .4 do Capítulo 2. Esses resultados são mostrados nas tabelas que seguem cada experimento. Observa-se que são mostrados apenas os valores acima da diagonal principal e que se o valor for positivo indica que o classificador na linha é melhor que o representado na coluna e se o valor encontrado for negativo, significa que o classificador indicado na coluna é melhor que o encontrado na linha.

Em alguns casos estes resultados foram ilustrados através de gráficos em que as barras representam as médias do Erro Total e as linhas sobre as barras representam o desvio padrão.

Como já mostrado no capítulo anterior, todos os experimentos são realizados com as ferramentas $S V M^{\text {light }}$ e Weka e uma comparação entre eles. Os resultados dos experimentos serão discutidos ao longo do texto.

\subsection{Resultados: Experimento 1 - DCan_set}

Os experimentos realizados com a identificação de atributos na forma canônica utilizando o etiquetador e o algoritmo baseado em (Mladenić 1998) foram denominados de Experimento 1. Observa-se que o script ScpDCan, gerou o conjunto de dados DCan_set.

\subsubsection{Sem Redução de features}

Na Tabela 6.1 são mostrados os resultados desse experimento, utilizando SVM com vários parâmetros, para representação booleana e freqüência. Ressalta-se que, neste caso, como mostrado na Tabela 5.3 do Capítulo 5, o número de features é de 13.849 .

Nos resultados deste experimento pode ser observado que o erro total foi o mesmo para todas as configurações do SVM quando utilizado a representação com atribuição de peso booleano. $\mathrm{Na}$ atribuição de peso por freqüência do atributo nos documentos, os valores se mantiveram constantes para o Kernel Radial Basis ( $\mathrm{t}=2)$, mesmo com a variação do parâmetro $\mathrm{g}$.

Neste experimento o menor erro total foi encontrado para a configuração default 


\begin{tabular}{|c|c|c|c|c|c|}
\hline & ס & (\%) & $\begin{array}{c}\text { erro da } \\
\text { classe }+(\%)\end{array}$ & $\begin{array}{l}\text { cobertura } \\
(\%)\end{array}$ & $\begin{array}{l}F_{1} \\
(\%)\end{array}$ \\
\hline $\begin{array}{l}\text { SVM } \\
(t=1 ; d=1)\end{array}$ & $\begin{array}{l}\text { Booleana } \\
\text { Frequiência }\end{array}$ & $\begin{array}{c}6,11 \pm 15,81 \\
5,83 \pm 8,09\end{array}$ & $\begin{array}{c}6,11 \pm 15,81 \\
3,11 \pm 6,89\end{array}$ & $\begin{array}{c}100 \pm 0,00 \\
95,50 \pm 7,53\end{array}$ & $\begin{array}{c}96,08 \pm 10,50 \\
96,08 \pm 6,41\end{array}$ \\
\hline $\begin{array}{l}\text { SVM } \\
(\mathrm{t}=1 ; \mathrm{d}=2)\end{array}$ & $\begin{array}{l}\text { Booleana } \\
\text { Freqüềncia }\end{array}$ & $\begin{array}{c}6,11 \pm 15,81 \\
8,37 \pm 9,45\end{array}$ & $\begin{array}{l}6,11 \pm 15,81 \\
3,25 \pm 7,08\end{array}$ & $\begin{array}{c}100 \pm 0,00 \\
92,82 \pm 7,87 \\
\end{array}$ & $\begin{array}{c}96,08 \pm 10,50 \\
94,65 \pm 6,81\end{array}$ \\
\hline $\begin{array}{l}\text { SVM } \\
(t=2 ; g=0,6)\end{array}$ & $\begin{array}{l}\text { Booleana } \\
\text { Frequiêncla }\end{array}$ & $\begin{array}{l}6,11 \pm 15,81 \\
6,98 \pm 14,20\end{array}$ & $\begin{array}{l}6,11 \pm 15,81 \\
5,55 \pm 14,10\end{array}$ & $\begin{array}{c}100 \pm 0,00 \\
98,57 \pm 4,52\end{array}$ & $\begin{array}{c}96,08 \pm 10,50 \\
95,79 \pm 9,02\end{array}$ \\
\hline $\begin{array}{l}\text { SVM } \\
(t=2 ; g=0,8)\end{array}$ & $\begin{array}{l}\text { Booleana } \\
\text { Freqüêncla }\end{array}$ & $\begin{array}{l}6,11 \pm 15,81 \\
6,98 \pm 14,20\end{array}$ & $\begin{array}{l}6,11 \pm 15,81 \\
5,55 \pm 14,10 \\
\end{array}$ & $\begin{array}{c}100 \pm 0,00 \\
98,57 \pm 4,52\end{array}$ & $\begin{array}{c}96,08 \pm 10,50 \\
95,79 \pm 9,02 \\
\end{array}$ \\
\hline $\begin{array}{l}\text { SVM } \\
(t=2 ; g=1,0)\end{array}$ & $\begin{array}{c}\text { Booleana } \\
\text { Frequência }\end{array}$ & $\begin{array}{l}6,11 \pm 15,81 \\
6,98 \pm 14,20\end{array}$ & $\begin{array}{l}6,11 \pm 15,81 \\
5,55 \pm 14,10\end{array}$ & $\begin{array}{c}100 \pm 0,00 \\
98,57 \pm 4,52\end{array}$ & $\begin{array}{r}96,08 \pm 10,50 \\
95,79 \pm 9,02\end{array}$ \\
\hline $\begin{array}{l}S V M \\
(t=2 ; g=1,2)\end{array}$ & $\begin{array}{l}\text { Booleana } \\
\text { Freqüêncla }\end{array}$ & $\begin{array}{l}6,11 \pm 15,81 \\
6,98 \pm 14,20\end{array}$ & $\begin{array}{l}6,11 \pm 15,81 \\
5,55 \pm 14,10\end{array}$ & $\begin{array}{c}100 \pm 0,00 \\
98,57 \pm 4,52\end{array}$ & $\begin{array}{c}96,08 \pm 10,50 \\
95,79 \pm 9,02\end{array}$ \\
\hline
\end{tabular}

Tabela 6.1: Experimento com DCan_set e algoritmo SVM, sem redução de features do SVM $(t=1 ; d=1)$ e representação por freqüência.

O erro da classe positiva teve um comportamento semelhante ao erro total, lembrando que nossos dados vieram de uma classe bivalorada, balanceada, como pode ser observado pela Tabela 5.1 do Capítulo 5 . Com relação à cobertura e $F_{1}$, não se pode tirar grandes conclusões, uma vez que a variação entre os dados foi muito pequena.

Calculando a diferença em desvios-padrão tem-se os resultados mostrados na Tabela 6.2 , com a representação booleana e de freqüência.

\begin{tabular}{|l||c|c|c|c|c|c|}
\hline \hline Parâmetros & $\begin{array}{c}t=1 ; d=1 \\
\text { Bool }\end{array}$ & $\begin{array}{c}t=1 ; d=1 \\
\text { Freq }\end{array}$ & $\begin{array}{c}t=1 ; d=2 \\
\text { Bool }\end{array}$ & $\begin{array}{c}t=1 ; d=2 \\
\text { Freq }\end{array}$ & $\begin{array}{c}t=2 ; g=0,6 \\
\text { Bool }\end{array}$ & $\begin{array}{c}t=2 ; g=0,6 \\
\text { Freq }\end{array}$ \\
\hline $\begin{array}{l}t=1 ; d=1 \\
\text { Bool }\end{array}$ & - & $-0,0223$ & 0 & 0,1735 & 0 & 0,0579 \\
\hline $\begin{array}{l}t=1 ; d=1 \\
\text { Freq }\end{array}$ & & - & 0,0223 & 0,2888 & 0,0223 & 0,0995 \\
\hline $\begin{array}{l}(t=1 ; d=2 \\
\text { Bool }\end{array}$ & & & - & 0,1735 & 0 & 0,0579 \\
\hline $\begin{array}{l}t=1 ; d=2 \\
\text { Freq }\end{array}$ & & & & - & $-0,1735$ & $-0,1152$ \\
\hline $\begin{array}{l}t=2 ; g=0,6 \\
\text { Bool }\end{array}$ & & & & & - & 0,0579 \\
\hline \hline
\end{tabular}

Tabela 6.2: Diferença em desvios-padrão com DCan_set e algoritmo SVM, sem redução de features

Na Tabela 6.2 não foi mostrada a comparação para SVM com os parâmetros 
$(t=2 ; g=0,8), t(t=2 ; g=1,0)$ e $(t=2 ; g=1,2)$ uma vez que os valores para o erro total eram iguais aos do parâmetro $(\mathrm{t}=2 ; \mathrm{g}=0,6)$, que foi utilizado, tanto para booleano quanto para freqüência.

Baseado nas diferenças em desvios-padrão o maior valor encontrado é $\mathbf{0 , 2 8 8 8}$. Esse valor indica que o SVM com parâmetros $(t=1 ; d=1)$ e representação de freqüência possui melhor desempenho quando comparado com SVM com parâmetro $(t=1 ; d=2)$ e representação de freqüência. Porém, observa-se neste experimento que não foi encontrado um classificador significativamente melhor que outro, com taxa de certeza $95 \%$.

O gráfico ilustrado na Figura 6.1 sintetiza os resultados obtidos com SVM e variaçōes de parâmetros, parcialmente mostrados na tabela anterior, utilizando DCan_set, sem redução de features. Neste caso pode ser observado que praticamente não houve diferenças significativas, ao se variar os parâmetros do SVM. Pode também ser observado que ocorreu um alto desvio padrão, para todo esse experimento.

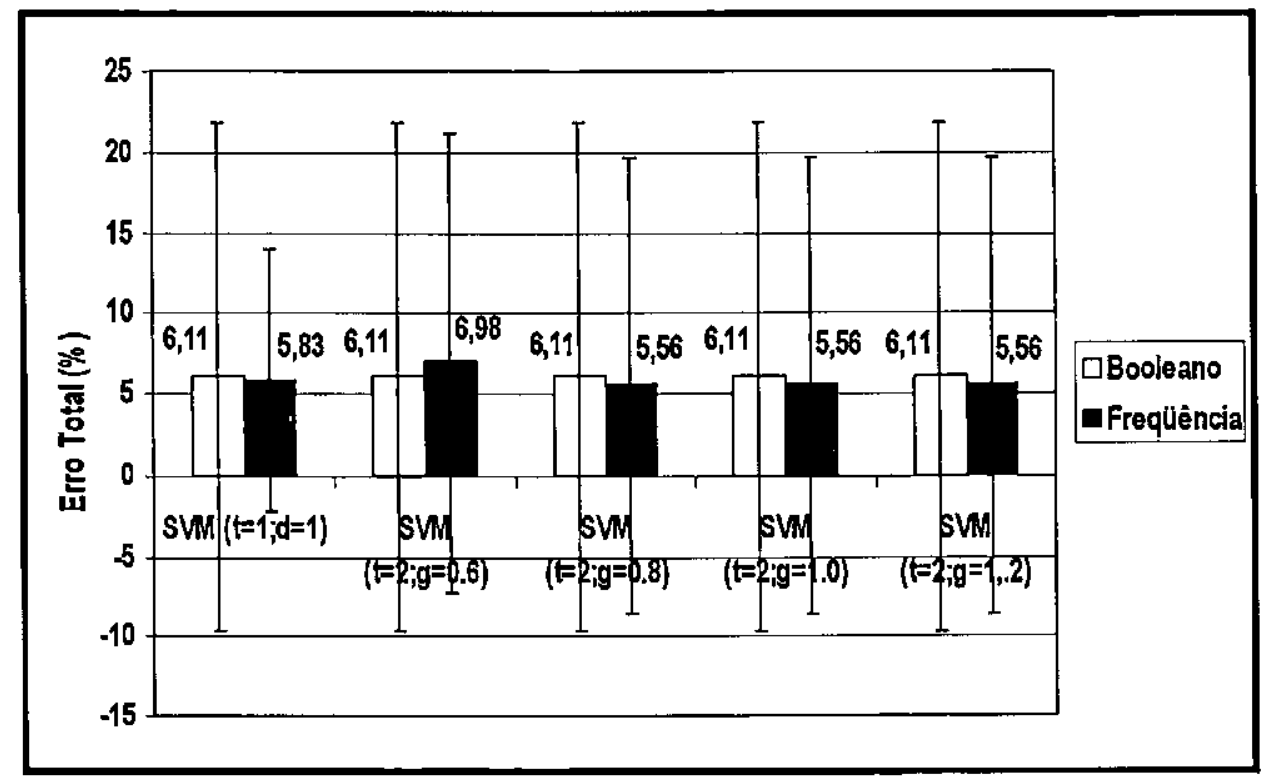

Figura 6.1: Comparaçōes dos resultados obtidos para o Erro Total com SVM e variações de parâmetros - DCan_set, sem redução de features 


\subsubsection{Redução para 250 features}

Na Tabela 6.3 são mostrados os resultados desse experimento, utilizando SVM com todos os parâmetros, para representação booleana e freqüência, com redução para 250 features.

\begin{tabular}{|c|c|c|c|c|c|}
\hline Algorltmo & Representaçāo & $\begin{array}{c}\text { erro total } \\
(\%)\end{array}$ & $\begin{array}{c}\text { erro da } \\
\text { classe }+(\%)\end{array}$ & $\begin{array}{c}\text { cobertura } \\
(\%)\end{array}$ & $\begin{array}{l}F_{1} \\
(\%)\end{array}$ \\
\hline $\begin{array}{l}\text { SVM } \\
(t=1 ; d=1)\end{array}$ & $\begin{array}{c}\text { Booleana } \\
\text { Frequiuêncla }\end{array}$ & $\begin{array}{l}5,78 \pm 14,79 \\
7,03 \pm 14,77\end{array}$ & $\begin{array}{l}5,78 \pm 14,79 \\
5,78 \pm 14,79\end{array}$ & $\begin{array}{c}100 \pm 0,00 \\
98,75 \pm 3,95\end{array}$ & $\begin{array}{l}96,37 \pm 9,60 \\
95,70 \pm 9,55\end{array}$ \\
\hline $\begin{array}{l}\text { SVM } \\
(t=1 ; d=2)\end{array}$ & $\begin{array}{c}\text { Booleana } \\
\text { Frequância }\end{array}$ & $\begin{array}{l}7,03 \pm 14,77 \\
8,81 \pm 17,38\end{array}$ & $\begin{array}{c}2,78 \pm 6,00 \\
6,25 \pm 15,87\end{array}$ & $\begin{array}{l}98,75 \pm 3,95 \\
96,25 \pm 6,04\end{array}$ & $\begin{array}{r}97,84 \pm 3,57 \\
94,45 \pm 11,62\end{array}$ \\
\hline $\begin{array}{l}\text { SVM } \\
(t=2 ; g=0,6)\end{array}$ & $\begin{array}{l}\text { Booleana } \\
\text { Frequância }\end{array}$ & $\begin{array}{l}5,78 \pm 14,79 \\
7,21 \pm 14,86\end{array}$ & $\begin{array}{l}5,78 \pm 14,79 \\
5,78 \pm 14,79\end{array}$ & $\begin{array}{c}100 \pm 0,00 \\
94,22 \pm 14,79\end{array}$ & $\begin{array}{l}96,37 \pm 9,60 \\
95,60 \pm 9,58\end{array}$ \\
\hline $\begin{array}{l}\text { SVM } \\
(t=2 ; g=0,8)\end{array}$ & $\begin{array}{c}\text { Booleana } \\
\text { Freqüuencla }\end{array}$ & $\begin{array}{l}5,78 \pm 14,79 \\
5,78 \pm 14,79 \\
\end{array}$ & $\begin{array}{l}5,78 \pm 14,79 \\
5,78 \pm 14,79 \\
\end{array}$ & $\begin{array}{c}100 \pm 0,00 \\
98,57 \pm 4,52\end{array}$ & $\begin{array}{l}96,37 \pm 9,60 \\
95,60 \pm 9,58 \\
\end{array}$ \\
\hline $\begin{array}{l}\text { SVM } \\
(t=2 ; g=1,0)\end{array}$ & $\begin{array}{l}\text { Booleana } \\
\text { Frequencia }\end{array}$ & $\begin{array}{l}5,78 \pm 14,79 \\
5,78 \pm 14,79\end{array}$ & $\begin{array}{l}5,78 \pm 14,79 \\
5,78 \pm 14,79\end{array}$ & $\begin{array}{c}100 \pm 0,00 \\
98,57 \pm 4,52\end{array}$ & $\begin{array}{l}96,37 \pm 9,60 \\
95,60 \pm 9,58 \\
\end{array}$ \\
\hline $\begin{array}{l}\text { SVM } \\
(t=2 ; g=1,2)\end{array}$ & $\begin{array}{c}\text { Booleana } \\
\text { Frequiencla }\end{array}$ & $\begin{array}{l}5,78 \pm 14,79 \\
5,78 \pm 14,79\end{array}$ & $\begin{array}{l}5,78 \pm 14,79 \\
5,78 \pm 14,75\end{array}$ & $\begin{array}{c}100 \pm 0,00 \\
98,57 \pm 4,52\end{array}$ & $\begin{array}{l}96,37 \pm 9,60 \\
95,60 \pm 9,58\end{array}$ \\
\hline
\end{tabular}

Tabela 6.3: Experimento com DCan_set e algoritmo SVM, com redução para 250 features

Observa-se que apesar da reduçāo para 250 features corresponder a 1,8\% do número total de atributos, os erro não foram tão diferentes. Por exemplo, calculando-se a diferença em desvios-padrão dos erros do resultado com menor erro total do experimento sem redução de features $(5,83 \pm 8,09)$ e o pior caso da redução para 250 features $(8,81 \pm$ 17,38). O resultado dessa diferença de desvios-padrāo é $\mathbf{0 , 2 1 9 8}$, que indica que um não é significativamente melhor que outro com taxa de certeza $95 \%$.

Calculando a diferença em desvios-padrão temos os resultados mostrados na Tabela 6.4, com a representação booleana e de freqüência. Baseado nessas diferenças o maior

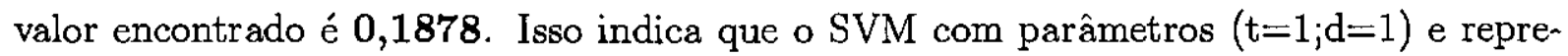
sentação booleana, possui desempenho levemente melhor quando comparado com SVM com parâmetros $(t=1 ; d=2)$ e representaçāo de freqüência. Esse mesmo valor também foi encontrado com outras comparações.

Esses classificadores são os que possuem maior diferença comparativa considerando o erro total e o desvio padrão, o que indica que todós os classificadores obtidos com SVM neste conjunto de documentos para esta representação de atributos tiveram resultados muito próximos com relação a precisão. 


\begin{tabular}{|c|c|c|c|c|c|c|c|c|}
\hline Parâmetros & $\begin{array}{c}\mathrm{t}=1 ; \mathrm{d}=1 \\
\text { Bool }\end{array}$ & $\begin{array}{c}\mathrm{t}=1 ; \mathrm{d}=1 \\
\text { Freq }\end{array}$ & $\begin{array}{c}t=1 ; d=2 \\
\text { Bool }\end{array}$ & $\begin{array}{c}t=1 ; d=2 \\
\text { Freq }\end{array}$ & $\begin{array}{c}\mathrm{t}=2 ; \mathrm{g}=0,6 \\
\text { Bool }\end{array}$ & $\begin{array}{c}\mathbf{t}=2 ; \mathrm{g}=0,6 \\
\text { Freq }\end{array}$ & $\begin{array}{c}\mathrm{t}=2 ; \mathrm{g}=0,8 \\
\text { Bool }\end{array}$ & $\begin{array}{c}t=2 ; g=0,8 \\
\text { Freq }\end{array}$ \\
\hline $\begin{array}{l}t=1 ; d=1 \\
\text { Bool }\end{array}$ & - & 0,0846 & 0 & 0,1878 & 0 & 0,0965 & 0 & 0 \\
\hline $\begin{array}{l}t=1 ; d=1 \\
\text { Freq }\end{array}$ & & - & $-0,0846$ & 0,1104 & $-0,0846$ & 0,0121 & $-0,0846$ & $-0,0846$ \\
\hline $\begin{array}{l}t=1 ; d=2 \\
\text { Bool }\end{array}$ & & & - & 0,1878 & 0 & 0,0965 & 0 & 0 \\
\hline $\begin{array}{l}t=1 ; d=2 \\
\text { Freq }\end{array}$ & & & & - & $-0,1878$ & $-0,0990$ & $-0,1878$ & $-0,1878$ \\
\hline $\begin{array}{l}\mathrm{t}=2 ; \mathrm{g}=0,6 \\
\text { Bool }\end{array}$ & & & & & - & 0,0965 & 0 & 0 \\
\hline $\begin{array}{l}t=2 ; g=0,6 \\
\text { Freq }\end{array}$ & & & & & & - & $-0,0965$ & $-0,0965$ \\
\hline $\begin{array}{l}t=2 ; g=0,8 \\
\text { Bool }\end{array}$ & & & & & & & - & 0 \\
\hline
\end{tabular}

Tabela 6.4: Diferença em desvios-padrão com DCan_set e algoritmo SVM, com redução para 250 features

Na Tabela 6.4 não foi mostrada a comparação para SVM com os parâmetros $(\mathrm{t}=2 ; \mathrm{g}=1,0)$ e $(\mathrm{t}=2 ; \mathrm{g}=1,2)$ uma vez que os valores para o erro total eram iguais aos do parâmetro $(t=2 ; g=0,8)$, que foi utilizado, tanto para booleano quanto para freqüência. 


\subsubsection{Redução para 500 features}

Na Tabela 6.5 são mostrados os resultados desse experimento, utilizando SVM com todos os parâmetros, para representação booleana e freqüência.

\begin{tabular}{|c|c|c|c|c|c|}
\hline Algoritmo & Representaçāo & $\begin{array}{c}\text { erro total } \\
(\%)\end{array}$ & $\begin{array}{c}\text { erro da } \\
\text { classe }+(\%)\end{array}$ & $\begin{array}{c}\text { cobertura } \\
(\%)\end{array}$ & $\begin{array}{c}F_{1} \\
(\%)\end{array}$ \\
\hline $\begin{array}{l}\text { SVM } \\
(t=1 ; d=1)\end{array}$ & $\begin{array}{l}\text { Booleana } \\
\text { Freqitêncla }\end{array}$ & $\begin{array}{l}2,22 \pm 4,68 \\
4,58 \pm 7,91\end{array}$ & $\begin{array}{l}2,78 \pm 6,00 \\
3,11 \pm 6,89\end{array}$ & $\begin{array}{c}100 \pm 0,00 \\
96,75 \pm 7,08\end{array}$ & $\begin{array}{l}98,50 \pm 3,25 \\
96,75 \pm 6,44\end{array}$ \\
\hline $\begin{array}{l}\text { SVM } \\
(t=1 ; d=2)\end{array}$ & $\begin{array}{c}\text { Booleana } \\
\text { Freqüêncla }\end{array}$ & $\begin{array}{c}2,22 \pm 4,68 \\
8,23 \pm 11,96\end{array}$ & $\begin{array}{l}2,78 \pm 6,00 \\
3,75 \pm 8,44 \\
\end{array}$ & $\begin{array}{c}100 \pm 0,00 \\
92,07 \pm 12,86\end{array}$ & $\begin{array}{c}98,50 \pm 3,25 \\
93,98 \pm 10,59\end{array}$ \\
\hline $\begin{array}{l}\text { SVM } \\
(\mathrm{t}=2 ; \mathrm{g}=0,6)\end{array}$ & $\begin{array}{l}\text { Booleana } \\
\text { Frequêncla }\end{array}$ & $\begin{array}{l}5,56 \pm 14,10 \\
6,98 \pm 14,20\end{array}$ & $\begin{array}{l}5,55 \pm 14,10 \\
5,55 \pm 14,10\end{array}$ & $\begin{array}{c}100 \pm 0,00 \\
98,57 \pm 4,52\end{array}$ & $\begin{array}{l}96,56 \pm 9,02 \\
95,79 \pm 9,02\end{array}$ \\
\hline $\begin{array}{l}\text { SVM } \\
(t=2 ; g=0,8)\end{array}$ & $\begin{array}{c}\text { Booleana } \\
\text { Frequêncla }\end{array}$ & $\begin{array}{l}5,56 \pm 14,10 \\
5,56 \pm 14,10\end{array}$ & $\begin{array}{l}5,55 \pm 14,10 \\
5,55 \pm 14,10\end{array}$ & $\begin{array}{c}100 \pm 0,00 \\
98,57 \pm 4,52\end{array}$ & $\begin{array}{l}96,56 \pm 9,02 \\
95,79 \pm 9,02\end{array}$ \\
\hline $\begin{array}{l}\text { SVM } \\
(t=2 ; g=1,0)\end{array}$ & $\begin{array}{l}\text { Booleana } \\
\text { Freqüêncla }\end{array}$ & $\begin{array}{l}5,56 \pm 14,10 \\
5,56 \pm 14,10\end{array}$ & $\begin{array}{l}5,55 \pm 14,10 \\
5,55 \pm 14,10\end{array}$ & $\begin{array}{c}100 \pm 0,00 \\
98,57 \pm 4,52\end{array}$ & $\begin{array}{l}96,56 \pm 9,02 \\
95,79 \pm 9,02\end{array}$ \\
\hline $\begin{array}{l}\text { SVM } \\
(t=2 ; g=1,2)\end{array}$ & $\begin{array}{c}\text { Booleana } \\
\text { Frequêencla }\end{array}$ & $\begin{array}{l}5,56 \pm 14,10 \\
5,56 \pm 14,10\end{array}$ & $\begin{array}{l}5,55 \pm 14,10 \\
5,55 \pm 14,10\end{array}$ & $\begin{array}{c}100 \pm 0,00 \\
98,57 \pm 4,52\end{array}$ & $\begin{array}{l}96,56 \pm 9,02 \\
95,79 \pm 9,02\end{array}$ \\
\hline
\end{tabular}

Tabela 6.5: Experimento com DCan_set e algoritmo SVM, com redução para 500 features

A redução para 500 features corresponde a 3,6\% do número total de atributos. Pode-se observar que a utilização de redução para 500 features gerou classificadores melhores que a redução para 250 features, mostrada anteriormente.

Calculando a diferença em desvios-padrão temos os resultados mostrados na Tabela 6.6, com a representação booleana e de freqüência. Baseado nas diferenças em desviospadrão o maior valor encontrado é 0,6618 que indica que o SVM com parâmetros $(t=1 ; d=1)$ e representação booleana, possui melhor desempenho quando comparado com SVM com parâmetros $(t=1 ; d=2)$ e representação de freqüência.

Comparando-se todos os kernels, tem-se a Figura 6.2, referente aos dados da Tabela 6.5 .

Nesta redução para 500 features também foram realizados experimentos com os algoritmos disponíveis na ferramenta Weka.

$\mathrm{Na}$ Tabela 6.7 são mostrados todos os resultados comparativos para os algoritmos da ferramenta Weka, utilizando-se de DCan_set e redução para 500 features.

Calculando a diferença em desvios-padrão temos os resultados mostrados nas Tabelas 6.8 e 6.9, para DCan_set com o algoritmo SVM $(t=1 ; d=1)$, que gerou melhor classifi- 


\begin{tabular}{|c|c|c|c|c|c|c|c|c|}
\hline Parâmetros & $\begin{array}{c}t=1 ; d=1 \\
\text { Bool }\end{array}$ & $\begin{array}{c}\mathrm{t}=1 ; \mathrm{d}= \\
\text { Froq }\end{array}$ & $\begin{array}{c}t=1 ; d=2 \\
\text { Bool }\end{array}$ & $\begin{array}{c}t=1 ; d=2 \\
\text { Freq }\end{array}$ & $\begin{array}{c}t=2 ; g=0,6 \\
\text { Bool }\end{array}$ & $\begin{array}{c}t=2 ; g=0,6 \\
\text { Freq }\end{array}$ & $\begin{array}{c}t=2 ; g=0,8 \\
\text { Bool }\end{array}$ & $\begin{array}{c}\mathrm{t}=2 ; \mathrm{g}=0,8 \\
\text { Freq }\end{array}$ \\
\hline $\begin{array}{l}t=1 ; d=1 \\
\text { Bool }\end{array}$ & - & 0,3631 & 0 & 0,6618 & 0,3179 & 0,4502 & 0,3179 & 0,3179 \\
\hline $\begin{array}{l}t=1 ; d=1 \\
\text { Freq }\end{array}$ & & - & $-0,3631$ & 0,3600 & 0,0857 & 0,2088 & 0,0857 & 0,0857 \\
\hline $\begin{array}{l}t=1 ; d=2 \\
\text { Bool }\end{array}$ & & & - & 0,6618 & 0,3179 & 0,4502 & 0,3179 & 0,3179 \\
\hline $\begin{array}{l}t=1 ; d=2 \\
\text { Freq }\end{array}$ & & & & - & $-0,2042$ & $-0,0952$ & $-0,2042$ & $-0,2042$ \\
\hline $\begin{array}{l}t=2 ; g=0,6 \\
\text { Bool }\end{array}$ & & & & & - & 0,1003 & 0 & 0 \\
\hline $\begin{array}{l}t=2 ; g=0,6 \\
\text { Freq }\end{array}$ & & & & & & - & $-0,1003$ & $-0,1003$ \\
\hline $\begin{array}{l}t=2 ; g=0,8 \\
\text { Bool }\end{array}$ & & & & & & & - & 0 \\
\hline
\end{tabular}

Tabela 6.6: Diferença em desvios-padrão com DCan_set e algoritmo SVM, com redução para 500 features

cador entre as diferentes configurações do SVM e os algoritmos da ferramenta Weka, com a representação booleana e de freqüência.

Baseado nas diferenças em desvios-padrão o maior valor encontrado é 0,3631, como mostrado na Tabela 6.8. Tanto o SVM com parâmetros $(\mathrm{t}=1 ; \mathrm{d}=1)$ e representação booleana quanto a Tabela de Decisão com representação de freqüência possuem melhor desempenho que os outros.

Baseado nas diferenças em desvios-padrão o maior valor encontrado é -1,0092, como mostrado na Tabela 6.9. A Tabela de Decisão com representação de freqüência possui melhor desempenho que todos outros, sendo que a maior diferença é encontrada com Naive Bayes.

O gráfico da Figura 6.3 ilustra os resultados obtidos com a melhor opção do SVM e os algoritmos da Weka, utilizando DCan.set.

Observando-se o gráfico da Figura 6.2 e o da Figura 6.3 percebe-se que para a representação booleana não houve grandes variações entre as taxas de erro dos classificadores gerados usando as duas ferramentas, enquanto que na representação de freqüência ocorreu uma variação bem maior. 


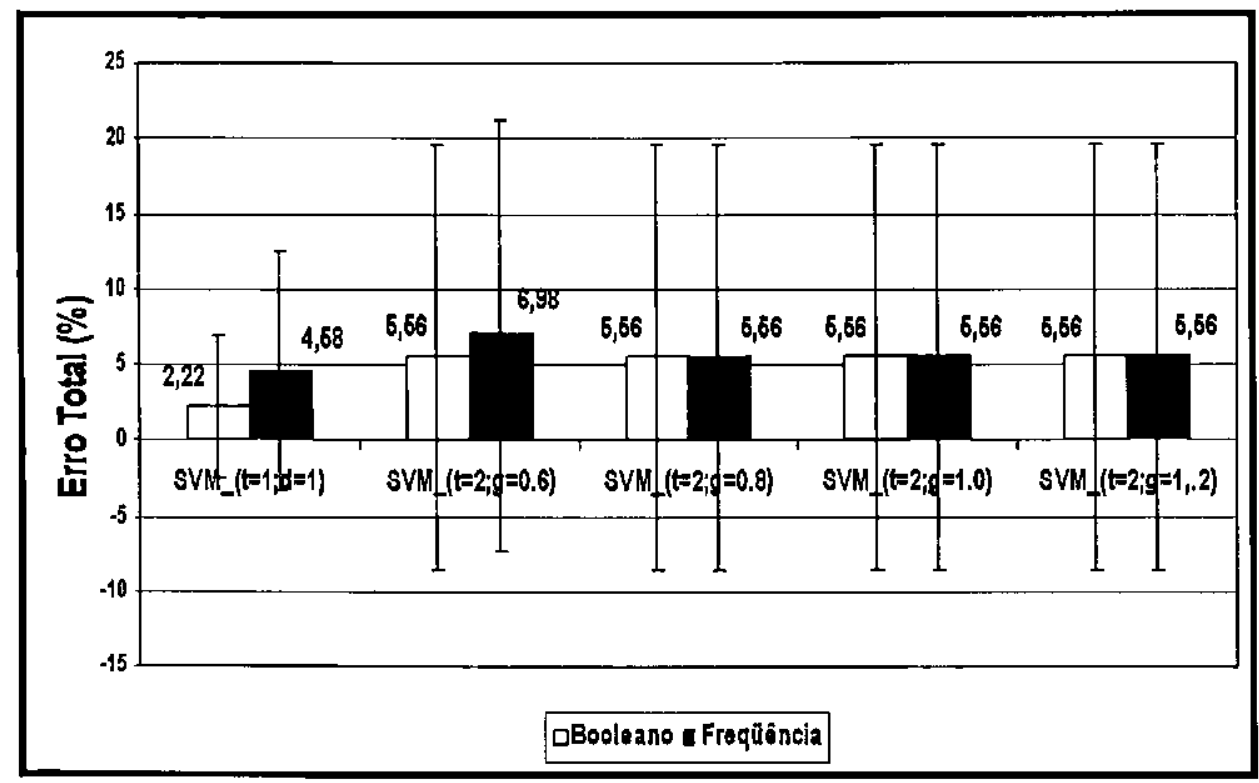

Figura 6.2: Comparações dos resultados obtidos (erro total) com SVM e variações de parâmetros - DCan_set, com redução para 500 features

\begin{tabular}{|c|c|c|c|c|c|}
\hline Algoritmo & Representação & $\begin{array}{c}\text { erro total } \\
(\%)\end{array}$ & $\begin{array}{c}\text { erro da } \\
\text { classe }+(\%)\end{array}$ & $\begin{array}{c}\text { cobertura } \\
(\%)\end{array}$ & $\begin{array}{r}F_{1} \\
(\%)\end{array}$ \\
\hline $\begin{array}{l}\text { SVM } \\
(t=1 ; d=1)\end{array}$ & $\begin{array}{l}\text { Booleana } \\
\text { Frequî̂ncia }\end{array}$ & $\begin{array}{l}2,22 \pm 4,68 \\
4,58 \pm 7,91\end{array}$ & $\begin{array}{l}2,78 \pm 6,00 \\
3,11 \pm 6,89\end{array}$ & $\begin{array}{c}100 \pm 0,00 \\
96,75 \pm 7,08\end{array}$ & $\begin{array}{l}98,50 \pm 3,25 \\
96,75 \pm 6,44\end{array}$ \\
\hline $\begin{array}{l}\text { Naive } \\
\text { Bayes }\end{array}$ & $\begin{array}{c}\text { Booleana } \\
\text { Frequiência }\end{array}$ & $\begin{array}{c}5,78 \pm 14,79 \\
13,10 \pm 14,51\end{array}$ & $\begin{array}{l}5,78 \pm 14,79 \\
5,69 \pm 14,16\end{array}$ & $\begin{array}{c}100 \pm 0,00 \\
92,32 \pm 8,87\end{array}$ & $\begin{array}{l}96,37 \pm 9,60 \\
92,36 \pm 9,07\end{array}$ \\
\hline $\begin{array}{l}\text { Vizinho } \\
\text { mais Próximo }\end{array}$ & $\begin{array}{l}\text { Booleana } \\
\text { Freqüência }\end{array}$ & $\begin{array}{l}5,78 \pm 14,79 \\
5,56 \pm 14,10\end{array}$ & $\begin{array}{l}5,78 \pm 14,79 \\
5,55 \pm 14,09\end{array}$ & $\begin{array}{l}100 \pm 0,00 \\
100 \pm 0,00\end{array}$ & $\begin{array}{l}96,37 \pm 9,61 \\
96,56 \pm 9,01\end{array}$ \\
\hline $\begin{array}{l}\text { Árvore } \\
\text { de Decisäo }\end{array}$ & $\begin{array}{l}\text { Booleana } \\
\text { Frequêncla }\end{array}$ & $\begin{array}{c}7,21 \pm 14,85 \\
8,23 \pm 14,07\end{array}$ & $\begin{array}{c}5,78 \pm 14,79 \\
5,55 \pm 14,09\end{array}$ & $\begin{array}{l}98,57 \pm 4,52 \\
97,32 \pm 5,67\end{array}$ & $\begin{array}{l}95,60 \pm 9,59 \\
95,12 \pm 8,91\end{array}$ \\
\hline $\begin{array}{l}\text { Regras } \\
\text { de Declsão }\end{array}$ & $\begin{array}{c}\text { Booleana } \\
\text { Frequêencia }\end{array}$ & $\begin{array}{c}5,78 \pm 14,79 \\
3,47 \pm 5,60\end{array}$ & $\begin{array}{c}5,78 \pm 14,79 \\
1,11 \pm 3,51\end{array}$ & $\begin{array}{c}100 \pm 0,00 \\
96,75 \pm 7,08 \\
\end{array}$ & $\begin{array}{l}96,37 \pm 9,61 \\
97,63 \pm 4,03\end{array}$ \\
\hline $\begin{array}{l}\text { Tabela } \\
\text { de Declsão }\end{array}$ & $\begin{array}{c}\text { Booleana } \\
\text { Freqüêncla }\end{array}$ & $\begin{array}{c}5,78 \pm 14,79 \\
2,22 \pm 4,68\end{array}$ & $\begin{array}{c}5,78 \pm 14,79 \\
1,11 \pm 3,15\end{array}$ & $\begin{array}{c}100 \pm 0,00 \\
96,57 \pm 7,35\end{array}$ & $\begin{array}{l}96,37 \pm 9,61 \\
97,53 \pm 4,17\end{array}$ \\
\hline
\end{tabular}

Tabela 6.7: Experimento com DCan_set usando a ferramenta Weka com os diferentes algoritmos e o algoritmo SVM, com redução para 500 features 


\begin{tabular}{|l||c|c|c|c|c|c|}
\hline \hline Algoritmo & $\begin{array}{c}\text { SVM } \\
(t=1 ; d=1) \\
\text { Bool }\end{array}$ & $\begin{array}{c}\text { SVM } \\
(t=1 ; d=1) \\
\text { Freq }\end{array}$ & $\begin{array}{c}\text { Regra de } \\
\text { Decisāo } \\
\text { Bool }\end{array}$ & $\begin{array}{c}\text { Regra de } \\
\text { Decisão } \\
\text { Freq }\end{array}$ & $\begin{array}{c}\text { Tabela de } \\
\text { Declsāo } \\
\text { Bool }\end{array}$ & $\begin{array}{c}\text { Tabela de } \\
\text { Decisão } \\
\text { Freq }\end{array}$ \\
\hline \hline $\begin{array}{l}\text { SVM( } t=1 ; d=1) \\
\text { Bool }\end{array}$ & - & 0,3631 & 0,3245 & 0,2422 & 0,3245 & 0 \\
\hline $\begin{array}{l}\text { SVM( } t=1 ; d=1) \\
\text { Freq }\end{array}$ & & - & 0,1012 & $-0,1620$ & 0,1012 & $-0,3631$ \\
\hline $\begin{array}{l}\text { Regra de Decisāo } \\
\text { Bool }\end{array}$ & & & - & $-0,2066$ & 0 & $-0,3245$ \\
\hline $\begin{array}{l}\text { Regra de Decisão } \\
\text { Freq }\end{array}$ & & & & - & 0,2066 & $-0,2422$ \\
\hline $\begin{array}{l}\text { Tabela de Decisão } \\
\text { Bool }\end{array}$ & & & & & - & $-0,3245$ \\
\hline \hline
\end{tabular}

Tabela 6.8: Diferença em desvios-padrão com DCan_set, algoritmo SVM e os algoritmos da Weka, com redução para 500 features

\begin{tabular}{|c|c|c|c|c|c|c|c|c|}
\hline Algoritmo & $\begin{array}{l}\text { Nalve } \\
\text { Bayes } \\
\text { Bool }\end{array}$ & $\begin{array}{l}\text { Nalve } \\
\text { Bayes } \\
\text { Freq }\end{array}$ & $\begin{array}{c}\text { Vizinho } \\
+ \text { Próximo } \\
\text { Bool }\end{array}$ & $\begin{array}{c}\text { Vizinho } \\
+ \text { Próximo } \\
\text { Freq }\end{array}$ & $\begin{array}{c}\text { Árvore } \\
\text { Decisão } \\
\text { Bool }\end{array}$ & $\begin{array}{c}\text { Árvore } \\
\text { Decísão } \\
\text { Freq }\end{array}$ & $\begin{array}{c}\text { Tabela de } \\
\text { Decisão } \\
\text { Bool }\end{array}$ & $\begin{array}{c}\text { Tabela de } \\
\text { Decisão } \\
\text { Freq }\end{array}$ \\
\hline $\begin{array}{l}\text { Nalve } \\
\text { Bool }\end{array}$ & - & 0,4996 & 0 & $-0,0152$ & 0,0965 & 0,1697 & 0 & $-0,3245$ \\
\hline $\begin{array}{l}\text { Nalve } \\
\text { Freq }\end{array}$ & & - & $-0,4996$ & $-0,5270$ & $-0,4012$ & $-0,3408$ & $-0,4996$ & $-1,0092$ \\
\hline $\begin{array}{l}\text { Vizinho } \\
\text { Bool }\end{array}$ & & & - & $-0,0152$ & 0,0965 & 0,1697 & 0 & $-0,3245$ \\
\hline $\begin{array}{l}\text { Vizinho } \\
\text { Freq }\end{array}$ & & & & - & 0,1140 & 0,1896 & 0,0152 & $-0,3179$ \\
\hline $\begin{array}{l}\text { Árvore } \\
\text { Bool }\end{array}$ & & & & & - & 0,0705 & $-0,0965$ & $-0,4532$ \\
\hline $\begin{array}{l}\text { Árvore } \\
\text { Freq }\end{array}$ & & & & & & - & $-0,1697$ & $-0,5732$ \\
\hline $\begin{array}{l}\text { Tabela } \\
\text { Bool }\end{array}$ & & & & & & & - & $-0,3245$ \\
\hline
\end{tabular}

Tabela 6.9: Diferença em desvios-padrão com DCan_set, algoritmo SVM e os algoritmos da Weka, com redução para 500 features 


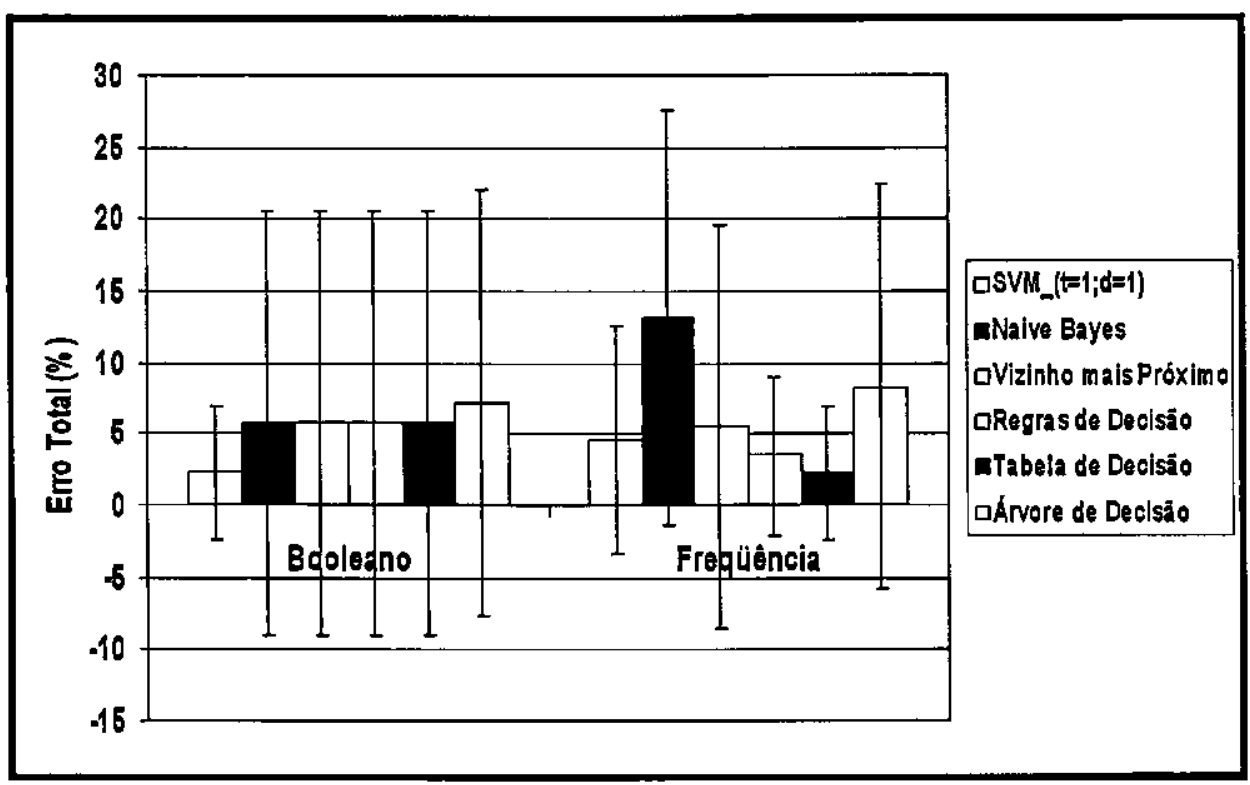

Figura 6.3: Comparações dos resultados do Erro Total do SVM e os algoritmos da ferramenta Weka usando DCan_set 


\subsubsection{Redução para 750 features}

Aplicou-se o script de redução para utilização do algoritmo SVM, com redução para 750 features. Na Tabela 6.10 são mostrados os resultados desse experimento, utilizando SVM com todos os parâmetros, para representação booleana e freqüência.

\begin{tabular}{|l||c|c|c|c|c|}
\hline \hline Algoritmo & Representaçāo & $\begin{array}{c}\text { erro total } \\
(\%)\end{array}$ & $\begin{array}{c}\text { erro da } \\
\text { classe }+(\%)\end{array}$ & $\begin{array}{c}\text { cobertura } \\
(\%)\end{array}$ & $\begin{array}{c}F_{1} \\
(\%)\end{array}$ \\
\hline \hline SVM & Booleana & $5,78 \pm 14,79$ & $5,78 \pm 14,79$ & $100 \pm 0,00$ & $96,37 \pm 9,60$ \\
$(t=1 ; d=1)$ & Frequiência & $8,28 \pm 14,64$ & $5,78 \pm 14,79$ & $97,50 \pm 5,27$ & $95,03 \pm 9,45$ \\
\hline SVM & Booleana & $5,78 \pm 14,79$ & $5,78 \pm 14,79$ & $100 \pm 0,00$ & $96,37 \pm 9,60$ \\
$(t=1 ; d=2)$ & Frequiêncla & $10,06 \pm 17,12$ & $6,25 \pm 15,87$ & $95,00 \pm 6,45$ & $93,78 \pm 11,45$ \\
\hline SVM & Booleana & $5,78 \pm 14,79$ & $5,78 \pm 14,79$ & $100 \pm 0,00$ & $96,37 \pm 9,60$ \\
$(t=2 ; g=0,6)$ & Freqüêncla & $7,21 \pm 14,86$ & $5,78 \pm 14,79$ & $98,57 \pm 4,52$ & $95,60 \pm 9,58$ \\
\hline SVM & Booleana & $5,78 \pm 14,79$ & $5,78 \pm 14,79$ & $100 \pm 0,00$ & $96,37 \pm 9,60$ \\
$(t=2 ; g=0,8)$ & Freqư̂ência & $5,78 \pm 14,79$ & $5,78 \pm 14,79$ & $98,57 \pm 4,52$ & $95,60 \pm 9,58$ \\
\hline SVM & Booleana & $5,78 \pm 14,79$ & $5,78 \pm 14,79$ & $100 \pm 0,00$ & $96,37 \pm 9,60$ \\
$(t=2 ; g=1,0)$ & Freqüência & $5,78 \pm 14,79$ & $5,78 \pm 14,79$ & $98,57 \pm 4,52$ & $95,60 \pm 9,58$ \\
\hline SVM & Booleana & $5,78 \pm 14,79$ & $5,78 \pm 14,79$ & $100 \pm 0,00$ & $96,37 \pm 9,60$ \\
$(t=2 ; g=1,2)$ & Freqừência & $5,78 \pm 14,79$ & $5,78 \pm 14,79$ & $98,57 \pm 4,52$ & $95,60 \pm 9,58$ \\
\hline \hline
\end{tabular}

Tabela 6.10: Experimento com DCan_set e algoritmo SVM, com reduçāo para 750 features

Neste experimento observou-se o mesmo padrão de comportamento do experimento com redução para 250 e 500 features, ou seja, todas as variações de configurações para representação booleana gerou a mesma taxa de erro, apesar da redução para 750 features corresponder a $5,4 \%$ do número total de atributos, uma percentagem maior que para as reduções anteriores.

Calculando a diferença em desvios-padrão temos os resultados mostrados na Tabela 6.11 , com a representação booleana e de freqüência. Baseado nas diferenças em desviospadrão o maior valor encontrado é 0,2675 , usando DCan_set com reduçāo para 750 features, indicando que o SVM com parâmetros $(t=1 ; d=2)$ e representação de freqüência possui melhor desempenho quando comparado com SVM com parâmetros $(t=1 ; d=2)$ e representação de freqüência. Esse mesmo valor foi encontrado com outras comparações também.

Comparando-se todos os resultados, tem-se o gráfico da Figura 6.4, referente a Tabela 6.10, que mostra os resultados obtidos com SVM e variações de parâmetros, uti- 


\begin{tabular}{|c|c|c|c|c|c|c|c|c|}
\hline Parâmetros & $\begin{array}{c}\mathrm{t}=1 ; \mathrm{d}=1 \\
\text { Bool }\end{array}$ & $\begin{array}{c}\mathbf{t}=\mathbf{1} ; \mathbf{d}=\mathbf{1} \\
\text { Freq }\end{array}$ & $\begin{array}{c}t=1 ; d=2 \\
\text { Bool }\end{array}$ & $\begin{array}{c}t=1 ; d=2 \\
\text { Freq }\end{array}$ & $\begin{array}{c}t=2 ; g=0,6 \\
\text { Bool }\end{array}$ & $\begin{array}{c}\mathrm{t}=2 ; \mathrm{g}=0,6 \\
\text { Freq }\end{array}$ & $\begin{array}{c}\mathrm{t}=2 ; \mathrm{g}=0,8 \\
\text { Bool }\end{array}$ & $\begin{array}{c}t=2 ; g=0,8 \\
\text { Freq }\end{array}$ \\
\hline $\begin{array}{l}t=1 ; d=1 \\
\text { Bool }\end{array}$ & - & 0,1699 & 0 & 0,2675 & 0 & 0,0965 & 0 & 0 \\
\hline $\begin{array}{l}t=1 ; d=1 \\
\text { Freq }\end{array}$ & & - & $-0,1699$ & 0,1118 & $-0,1699$ & $-0,0725$ & $-0,1699$ & $-0,1699$ \\
\hline $\begin{array}{l}t=1 ; d=2 \\
\text { Bool }\end{array}$ & & & - & 0,2675 & 0 & 0,0965 & 0 & 0 \\
\hline $\begin{array}{l}t=1 ; d=2 \\
\text { Freq }\end{array}$ & & & & - & $-0,2675$ & $-0,1778$ & $-0,2675$ & $-0,2675$ \\
\hline $\begin{array}{l}t=2 ; g=0,6 \\
\text { Bool }\end{array}$ & & & & & - & 0,0965 & 0 & 0 \\
\hline $\begin{array}{l}t=2 ; g=0,6 \\
\text { Freq }\end{array}$ & & & & & & - & $-0,0965$ & $-0,0965$ \\
\hline $\begin{array}{l}\mathrm{t}=2 ; \mathrm{g}=0,8 \\
\text { Bool }\end{array}$ & & & & & & & - & 0 \\
\hline
\end{tabular}

Tabela 6.11: Diferença em desvios-padrão com DCan_set e algoritmo SVM, com redução para 750 features

lizando DCan_set. 


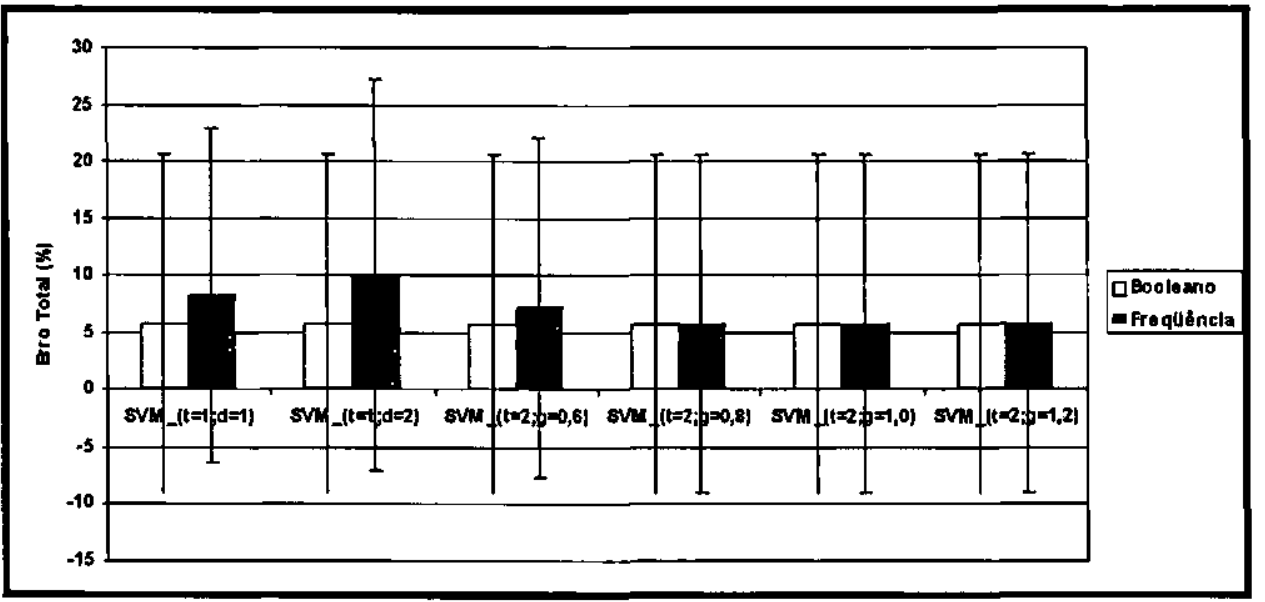

Figura 6.4: Comparações dos resultados obtidos (Erro Total) com SVM e variações de parâmetros com DCan_set e redução para 750 features 


\subsubsection{Redução para 1.000 features}

Aplicou-se o script de redução para utilização do algoritmo SVM, com redução para 1.000 features.

$\mathrm{Na}$ Tabela 6.12 são mostrados os resultados desse experimento, utilizando SVM, para representação booleana e freqüência.

\begin{tabular}{|c|c|c|c|c|c|}
\hline Algoritmo & Representaçāo & $\begin{array}{l}\text { erro total } \\
\qquad(\%)\end{array}$ & $\begin{array}{c}\text { erro da } \\
\text { classe }+(\%)\end{array}$ & $\begin{array}{c}\text { cobertura } \\
(\%)\end{array}$ & $\begin{array}{l}F_{1} \\
(\%)\end{array}$ \\
\hline $\begin{array}{l}\operatorname{SVM} \\
(\mathrm{t}=1 ; \mathrm{d}=1)\end{array}$ & $\begin{array}{l}\text { Booleana } \\
\text { Frequîencia }\end{array}$ & $\begin{array}{l}5,78 \pm 14,79 \\
8,28 \pm 14,64\end{array}$ & $\begin{array}{l}5,78 \pm 14,79 \\
5,78 \pm 14,79\end{array}$ & $\begin{array}{c}100 \pm 0,00 \\
97,50 \pm 5,27\end{array}$ & $\begin{array}{l}96,37 \pm 9,60 \\
95,03 \pm 9,45\end{array}$ \\
\hline $\begin{array}{l}\text { SVM } \\
(t=1 ; d=2)\end{array}$ & $\begin{array}{c}\text { Booleana } \\
\text { Freqüûncla }\end{array}$ & $\begin{array}{c}5,78 \pm 14,79 \\
11,48 \pm 16,79\end{array}$ & $\begin{array}{l}5,78 \pm 14,79 \\
6,78 \pm 14,79 \\
\end{array}$ & $\begin{array}{c}100 \pm 0,00 \\
93,57 \pm 6,80\end{array}$ & $\begin{array}{c}96,37 \pm 9,60 \\
93,01 \pm 11,25\end{array}$ \\
\hline $\begin{array}{l}\text { SVM } \\
(t=2 ; g=0,6)\end{array}$ & $\begin{array}{l}\text { Booleana } \\
\text { Freqüêncla }\end{array}$ & $\begin{array}{l}5,78 \pm 14,79 \\
7,21 \pm 14,86\end{array}$ & $\begin{array}{l}5,78 \pm 14,79 \\
5,78 \pm 14,79\end{array}$ & $\begin{array}{c}100 \pm 0,00 \\
98,57 \pm 4,52\end{array}$ & $\begin{array}{l}96,37 \pm 9,60 \\
95,40 \pm 9,58\end{array}$ \\
\hline $\begin{array}{l}\text { SVM } \\
(t=2 ; g=0,8)\end{array}$ & $\begin{array}{c}\text { Booleana } \\
\text { Frequitêncla }\end{array}$ & $\begin{array}{l}5,78 \pm 14,79 \\
5,78 \pm 14,79\end{array}$ & $\begin{array}{l}5,78 \pm 14,79 \\
5,78 \pm 14,79 \\
\end{array}$ & $\begin{array}{c}100 \pm 0,00 \\
98,57 \pm 4,52\end{array}$ & $\begin{array}{r}96,37 \pm 9,60 \\
95,60 \pm 9,58 \\
\end{array}$ \\
\hline $\begin{array}{l}\text { SVM } \\
(t=2 ; g=1,0)\end{array}$ & $\begin{array}{c}\text { Booleana } \\
\text { Freqüência }\end{array}$ & $\begin{array}{l}5,78 \pm 14,79 \\
5,78 \pm 14,79\end{array}$ & $\begin{array}{l}5,78 \pm 14,79 \\
5,78 \pm 14,79\end{array}$ & $\begin{array}{c}100 \pm 0,00 \\
98,57 \pm 4,52\end{array}$ & $\begin{array}{l}96,37 \pm 9,60 \\
95,60 \pm 9,58 \\
\end{array}$ \\
\hline $\begin{array}{l}\text { SVM } \\
(t=2 ; g=1,2)\end{array}$ & $\begin{array}{c}\text { Booleana } \\
\text { Freqüencia }\end{array}$ & $\begin{array}{l}5,78 \pm 14,79 \\
5,78 \pm 14,79\end{array}$ & $\begin{array}{l}5,78 \pm 14,79 \\
5,78 \pm 14,79\end{array}$ & $\begin{array}{c}100 \pm 0,00 \\
98,57 \pm 4,52\end{array}$ & $\begin{array}{l}96,37 \pm 9,60 \\
95,60 \pm 9,58\end{array}$ \\
\hline
\end{tabular}

Tabela 6.12: Experimento com DCan_set e algoritmo SVM, com redução para 1.000 features

A redução para 1.000 features corresponde a $7,2 \%$ do número total de atributos. Neste experimento pode ser observado que o erro total foi o mesmo para todas as configurações do SVM quando utilizado a representação por peso booleano. $\mathrm{Na}$ atribuição de peso por freqüência do atributo nos documentos, os valores se mantiveram constantes para o Kermel Radial Basis $(\mathrm{t}=2)$ com o parâmetro g de 0,8, 1,0 e 1,2.

Neste experimento o menor erro total foi encontrado para várias configurações, sendo esse valor constante para toda a representação booleana.

Com relação a cobertura e $F_{1}$ não pode se tirar grandes conclusões, uma vez que a variação entre os dados foi muito pequena, com exceção para SVM $(t=1 ; d=2)$ e representação por freqüência. Com relação ao erro da classe positiva também teve um comportamento semelhante ao erro total, sendo que na maioria dos casos igual ao erro total.

Calculando a diferença em desvios-padrão temos os resultados mostrados na Tabela 
6.13, com a representação booleana e de freqüência. Baseado nas diferenças em desviospadrão do erro total o maior valor encontrado é 0,3603 , usando DCan_set com redução de 1.000 features, indicando que o SVM com parâmetros $(t=1 ; d=1)$ e representação booleana possui melhor desempenho quando comparado com SVM com parâmetros $(t=1 ; d=2)$ e representação de freqüência. Esse mesmo valor foi encontrado com outras comparações também.

\begin{tabular}{|c|c|c|c|c|c|c|c|c|}
\hline Parâmetros & $\begin{array}{c}t=1 ; d=1 \\
\text { Bool }\end{array}$ & $\begin{array}{c}t=1 ; d=1 \\
\text { Freq }\end{array}$ & $\begin{array}{c}t=1 ; d=2 \\
\text { Bool }\end{array}$ & $\begin{array}{c}t=1 ; d=2 \\
\text { Freq }\end{array}$ & $\begin{array}{c}t=2 ; g=0,6 \\
\text { Bool }\end{array}$ & $\begin{array}{c}t=2 ; g=0,6 \\
\text { Freq }\end{array}$ & $\begin{array}{c}\mathrm{t}=2 ; \mathrm{g}=0,8 \\
\text { Bool }\end{array}$ & $\begin{array}{c}t=2 ; g=0,8 \\
\text { Freq }\end{array}$ \\
\hline $\begin{array}{l}t=1 ; d=1 \\
\text { Bool }\end{array}$ & - & 0,1699 & 0 & 0,3603 & 0 & 0,0965 & 0 & 0 \\
\hline $\begin{array}{l}t=1 ; d=1 \\
\text { Freq }\end{array}$ & & - & $-0,1699$ & 0,2032 & $-0,1699$ & $-0,0725$ & $-0,1699$ & $-0,1699$ \\
\hline $\begin{array}{l}t=1 ; d=2 \\
\text { Bool }\end{array}$ & & & - & 0,3603 & 0 & 0,0965 & 0 & 0 \\
\hline $\begin{array}{l}t=1 ; d=2 \\
\text { Freq }\end{array}$ & & & & - & $-0,3603$ & $-0,2693$ & $-0,3603$ & $-0,3603$ \\
\hline $\begin{array}{l}t=2 ; g=0,6 \\
\text { Bool }\end{array}$ & & & & & - & 0,0965 & 0 & 0 \\
\hline $\begin{array}{l}t=2 ; g=0,6 \\
\text { Freq }\end{array}$ & & & & & & - & $-0,0965$ & $-0,0965$ \\
\hline $\begin{array}{l}t=2 ; g=0,8 \\
\text { Bool }\end{array}$ & & & & & & & - & 0 \\
\hline
\end{tabular}

Tabela 6.13: Diferença em desvios-padrão com DCan_set e algoritmo SVM, com redução para 1.000 features

Comparando-se todos os kernels, tem-se o gráfico 6.5, referente a Tabela 6.12, que mostra os resultados obtidos com SVM e variações de parâmetros do SVM, utilizando DCan_Bool_set e DCan_Freq_set. 


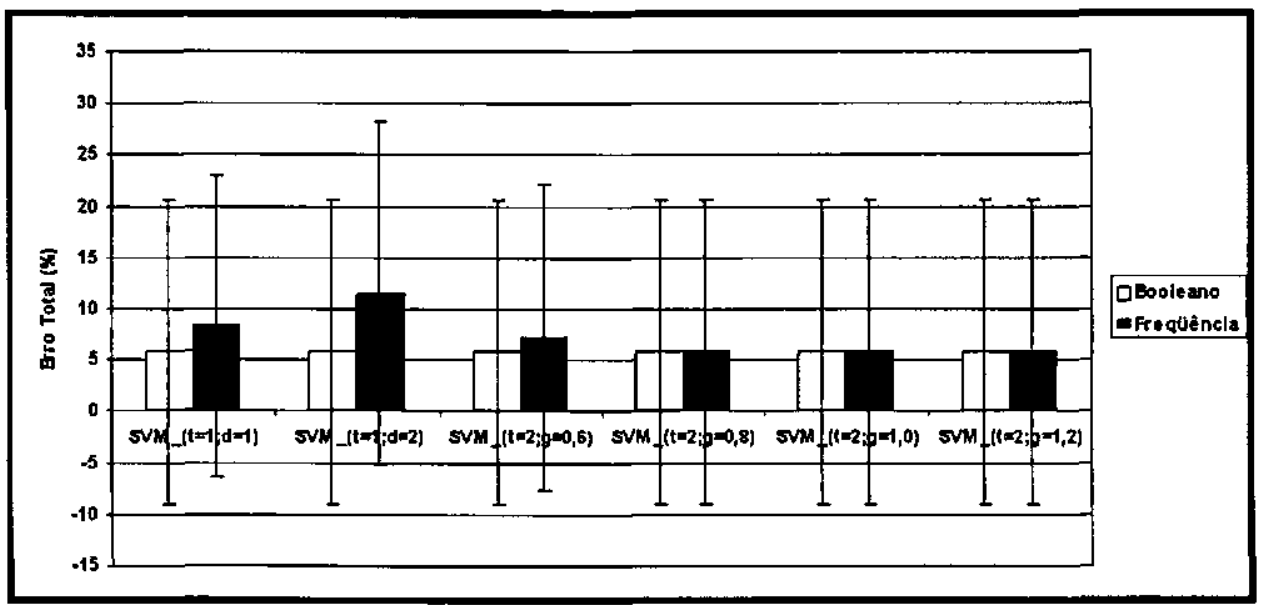

Figura 6.5: Comparações dos resultados obtidos (Erro Total) com SVM e variações de parâmetros, DCan_set e reduçāo para 1.000 features 


\subsubsection{Redução para 1.500 features}

Aplicou-se o script de redução para utilização do algoritmo SVM, com redução para 1.500 features.

$\mathrm{Na}$ Tabela 6.14 são mostrados os resultados desse experimento, utilizando SVM com todos os parâmetros, para representação booleana e freqüência.

\begin{tabular}{|c|c|c|c|c|c|}
\hline Algoritmo & Representação & $\begin{array}{c}\text { erro total } \\
(\%)\end{array}$ & $\begin{array}{c}\text { erro da } \\
\text { classe }+(\%)\end{array}$ & $\begin{array}{c}\text { cobertura } \\
(\%)\end{array}$ & $\begin{array}{l}F_{1} \\
(\%)\end{array}$ \\
\hline $\begin{array}{l}\text { SVM } \\
(t=1 ; d=1)\end{array}$ & $\begin{array}{c}\text { Booleana } \\
\text { Freqüëncla }\end{array}$ & $\begin{array}{l}5,78 \pm 14,79 \\
8,28 \pm 14,64\end{array}$ & $\begin{array}{l}5,78 \pm 14,79 \\
5,78 \pm 14,79\end{array}$ & $\begin{array}{c}100 \pm 0,00 \\
97,50 \pm 5,27\end{array}$ & $\begin{array}{l}96,37 \pm 9,60 \\
95,03 \pm 9,45\end{array}$ \\
\hline $\begin{array}{l}\text { SVM } \\
(t=1 ; d=2)\end{array}$ & $\begin{array}{l}\text { Booleana } \\
\text { Freqülencla }\end{array}$ & $\begin{array}{c}5,78 \pm 14,79 \\
11,48 \pm 16,79\end{array}$ & $\begin{array}{l}5,78 \pm 14,79 \\
6,78 \pm 14,79\end{array}$ & $\begin{array}{c}100 \pm 0,00 \\
93,57 \pm 6,80\end{array}$ & $\begin{array}{c}96,37 \pm 9,60 \\
93,01 \pm 11,25\end{array}$ \\
\hline $\begin{array}{l}\text { SVM } \\
(t=2 ; g=0,6)\end{array}$ & $\begin{array}{c}\text { Booleana } \\
\text { Frequêncla }\end{array}$ & $\begin{array}{l}5,78 \pm 14,79 \\
7,21 \pm 14,86\end{array}$ & $\begin{array}{l}5,78 \pm 14,79 \\
5,78 \pm 14,79\end{array}$ & $\begin{array}{c}100 \pm 0,00 \\
98,57 \pm 4,52\end{array}$ & $\begin{array}{l}96,37 \pm 9,60 \\
95,40 \pm 9,58\end{array}$ \\
\hline $\begin{array}{l}\text { SVM } \\
(t=2 ; g=0,8)\end{array}$ & $\begin{array}{c}\text { Booleana } \\
\text { Freqưuencla }\end{array}$ & $\begin{array}{l}5,78 \pm 14,79 \\
5,78 \pm 14,79\end{array}$ & $\begin{array}{l}5,78 \pm 14,79 \\
5,78 \pm 14,79\end{array}$ & $\begin{array}{c}100 \pm 0,00 \\
98,57 \pm 4,52\end{array}$ & $\begin{array}{l}96,37 \pm 9,60 \\
95,60 \pm 9,58\end{array}$ \\
\hline $\begin{array}{l}\text { SVM } \\
(t=2 ; g=1,0)\end{array}$ & $\begin{array}{c}\text { Booleana } \\
\text { Frequỉencla }\end{array}$ & $\begin{array}{l}5,78 \pm 14,79 \\
5,78 \pm 14,79\end{array}$ & $\begin{array}{l}\mathbf{5 , 7 8} \pm 14,79 \\
\mathbf{5 , 7 8} \pm 14,79\end{array}$ & $\begin{array}{c}100 \pm 0,00 \\
98,57 \pm 4,52\end{array}$ & $\begin{array}{l}96,37 \pm 9,60 \\
95,60 \pm 9,58\end{array}$ \\
\hline $\begin{array}{l}S V M \\
(t=2 ; g=1,2)\end{array}$ & $\begin{array}{c}\text { Booleana } \\
\text { Frequêncla }\end{array}$ & $\begin{array}{l}5,78 \pm 14,79 \\
5,78 \pm 14,79\end{array}$ & $\begin{array}{l}5,78 \pm 14,79 \\
5,78 \pm 14,79\end{array}$ & $\begin{array}{c}100 \pm 0,00 \\
98,57 \pm 4,52\end{array}$ & $\begin{array}{l}96,37 \pm 9,60 \\
95,60 \pm 9,58\end{array}$ \\
\hline
\end{tabular}

Tabela 6.14: Experimento com DCan_set e algoritmo SVM, com redução para 1.500 features

Observa-se a redução para 1.000 features corresponde a $10,8 \%$ do número total de atributos. Todo o comportamento deste experimento é muito semelhante a redução para 1.000 features, não trazendo nenhuma melhoria o aumento do número de atributos.

Neste experimento pode ser observado que o erro total foi o mesmo para todas as configurações do SVM quando utilizado a representação por peso booleano. Na atribuição de peso por freqüência do atributo nos documentos, os valores se mantiveram constantes para o Kernel Radial Basis ( $\mathrm{t}=2$ ) com o parâmetro $\mathrm{g}$ de 0,8, 1,0 e 1,2.

Neste experimento o menor erro total foi encontrado para várias configuraçōes, sendo esse valor constante para toda a representação booleana.

Com relação a cobertura e $F_{1}$ não pode se tirar grandes conclusões, uma vez que a variação entre os dados foi muito pequena, com exceção para SVM $(t=1 ; d=2)$ e representação por freqüência. Com relação ao erro da classe positiva também teve um 
comportamento semelhante ao erro total, sendo que na maioria dos casos igual ao erro total.

Calculando a diferença em desvios-padrão temos os resultados mostrados na Tabela 6.15 , com a representação booleana e de freqüência.

\begin{tabular}{|c|c|c|c|c|c|c|c|c|}
\hline Parâmetros & $\begin{array}{c}t=1 ; d=1 \\
\text { Bool }\end{array}$ & $\begin{array}{c}\mathrm{t}=1 ; \mathrm{d}=1 \\
\text { Freq }\end{array}$ & $\begin{array}{c}t=1 ; d=2 \\
\text { Bool }\end{array}$ & $\begin{array}{c}t=1 ; d=2 \\
\text { Freq }\end{array}$ & $\begin{array}{c}\mathrm{t}=2 ; \mathrm{g}=0,6 \\
\text { Bool }\end{array}$ & $\begin{array}{c}t=2 ; g=0,6 \\
\text { Freq }\end{array}$ & $\begin{array}{c}t=2 ; g=0,8 \\
\text { Bool }\end{array}$ & $\begin{array}{c}t=2 ; g=0,8 \\
\text { Freq }\end{array}$ \\
\hline $\begin{array}{l}t=1 ; d=1 \\
\text { Bool }\end{array}$ & - & 0,1699 & 0 & 0,3603 & 0 & 0,0965 & 0 & 0 \\
\hline $\begin{array}{l}t=1 ; d=1 \\
\text { Freq }\end{array}$ & & - & $-0,1699$ & 0,2032 & $-0,1699$ & $-0,0725$ & $-0,1699$ & $-0,1699$ \\
\hline $\begin{array}{l}t=1 ; d=2 \\
\text { Bool }\end{array}$ & & & - & 0,3603 & 0 & 0,0965 & 0 & 0 \\
\hline $\begin{array}{l}t=1 ; d=2 \\
\text { Freq }\end{array}$ & & & & - & $-0,3603$ & $-0,2693$ & $-0,3603$ & $-0,3603$ \\
\hline $\begin{array}{l}t=2 ; g=0,6 \\
\text { Bool }\end{array}$ & & & & & - & 0,0965 & 0 & 0 \\
\hline $\begin{array}{l}t=2 ; g=0,6 \\
\text { Freq }\end{array}$ & & & & & & - & $-0,0965$ & $-0,0965$ \\
\hline $\begin{array}{l}t=2 ; g=0,8 \\
\text { Bool }\end{array}$ & & & & & & & - & 0 \\
\hline
\end{tabular}

Tabela 6.15: Diferença em desvios-padrão com DCan_set e algoritmo SVM, com redução para 1.500 features

Baseado nas diferenças em desvios-padrão do erro total o maior valor encontrado é 0,3603, usando DCan_set com redução para 1.500 features, que indica que o SVM com parâmetros $(t=1 ; d=1)$ e representação booleana possui melhor desempenho quando comparado com SVM com parâmetros $(t=1 ; d=2)$ e representação de freqüência. Esse mesmo valor foi encontrado com outras comparações.

Comparando-se todos os kernels, tem-se o gráfico 6.6, referente a Tabela 6.14, que mostra os resultados obtidos com SVM e variações de parâmetros do SVM, utilizando DCan_set. 


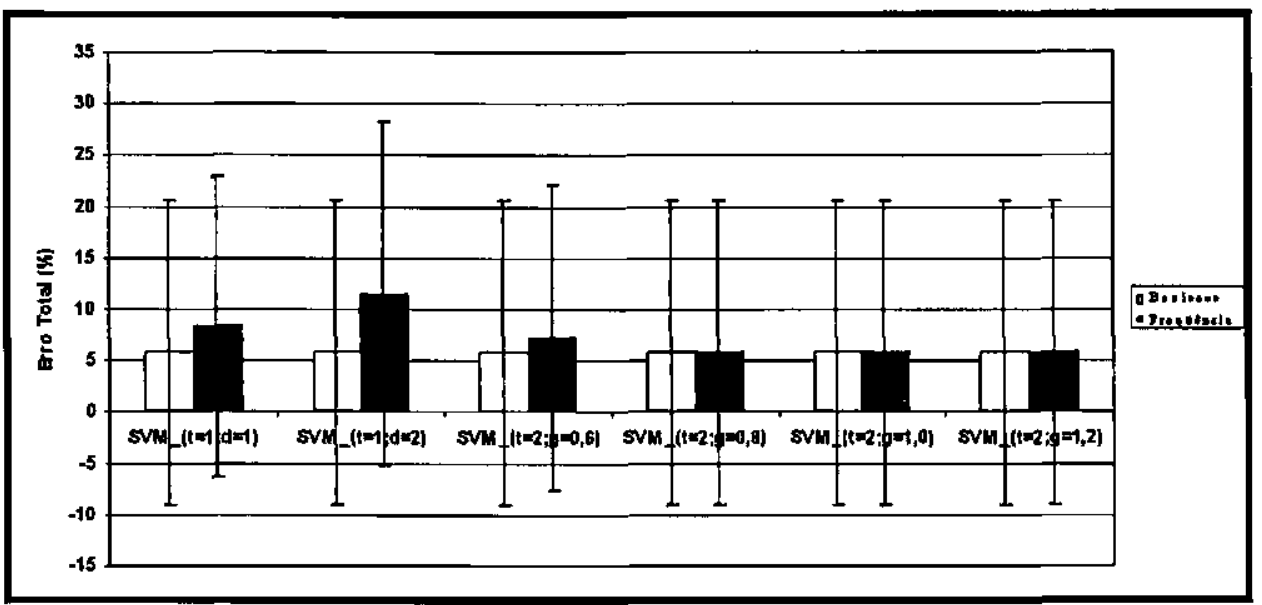

Figura 6.6: Comparaçōes dos resultados obtidos (Erro Total) com SVM e variaçōes de parâmetros, DCan_set e redução para 1.500 features 


\subsubsection{Redução para 3.000 features}

Na Tabela 6.16 são mostrados os resultados desse experimento, utilizando SVM com todos os parâmetros, para representação booleana e freqüência, com redução para 3.000 features.

\begin{tabular}{|c|c|c|c|c|c|}
\hline $\mathbf{A l g}$ & io & $\begin{array}{c}\text { erro total } \\
(\%)\end{array}$ & $\begin{array}{c}\text { erro da } \\
\text { classe }+(\%)\end{array}$ & $\begin{array}{c}\text { cobertura } \\
(\%)\end{array}$ & $\begin{array}{l}F_{1} \\
(\%)\end{array}$ \\
\hline $\begin{array}{l}\text { SVM } \\
(t=1 ; d=1)\end{array}$ & $\begin{array}{c}\text { Booleana } \\
\text { Frequiêncta }\end{array}$ & $\begin{array}{l}5,78 \pm 14,79 \\
7,03 \pm 14,77\end{array}$ & $\begin{array}{l}5,78 \pm 14,79 \\
5,78 \pm 14,79\end{array}$ & $\begin{array}{c}100 \pm 0,00 \\
96,75 \pm 7,08\end{array}$ & $\begin{array}{c}96,37 \pm 9,60 \\
95,14 \pm 11,25\end{array}$ \\
\hline $\begin{array}{l}\text { SVM } \\
(t=1 ; d=2)\end{array}$ & $\begin{array}{l}\text { Booleana } \\
\text { Freqüêncla }\end{array}$ & $\begin{array}{l}7,03 \pm 14,77 \\
8,81 \pm 17,38\end{array}$ & $\begin{array}{l}5,78 \pm 14,79 \\
6,25 \pm 15,87\end{array}$ & $\begin{array}{l}98,75 \pm 3,95 \\
96,25 \pm 6,04\end{array}$ & $\begin{array}{c}95,70 \pm 9,55 \\
94,45 \pm 11,62\end{array}$ \\
\hline $\begin{array}{l}\text { SVM } \\
(t=2 ; g=0,6)\end{array}$ & $\begin{array}{c}\text { Booleana } \\
\text { Frequîencla }\end{array}$ & $\begin{array}{l}5,78 \pm 14,79 \\
7,21 \pm 14,86 \\
\end{array}$ & $\begin{array}{l}5,78 \pm 14,79 \\
5,78 \pm 14,79\end{array}$ & $\begin{array}{c}100 \pm 0,00 \\
98,57 \pm 4,52\end{array}$ & $\begin{array}{l}96,37 \pm 9,60 \\
95,60 \pm 9,58\end{array}$ \\
\hline $\begin{array}{l}\text { SVM } \\
(t=2 ; g=0,8)\end{array}$ & $\begin{array}{l}\text { Booleana } \\
\text { Freqüuencla }\end{array}$ & $\begin{array}{l}5,78 \pm 14,79 \\
5,78 \pm 14,79 \\
\end{array}$ & $\begin{array}{l}5,78 \pm 14,79 \\
5,78 \pm 14,79 \\
\end{array}$ & $\begin{array}{c}100 \pm 0,00 \\
98,57 \pm 4,52\end{array}$ & $\begin{array}{l}96,37 \pm 9,60 \\
95,60 \pm 9,58 \\
\end{array}$ \\
\hline $\begin{array}{l}\text { SVM } \\
(t=2 ; g=1,0)\end{array}$ & $\begin{array}{l}\text { Booleana } \\
\text { Freqüêncla }\end{array}$ & $\begin{array}{l}5,78 \pm 14,79 \\
5,78 \pm 14,79\end{array}$ & $\begin{array}{l}5,78 \pm 14,79 \\
5,78 \pm 14,79\end{array}$ & $\begin{array}{c}100 \pm 0,00 \\
98,57 \pm 4,52\end{array}$ & $\begin{array}{l}96,37 \pm 9,60 \\
95,60 \pm 9,58\end{array}$ \\
\hline $\begin{array}{l}\text { SVM } \\
(t=2 ; g=1,2)\end{array}$ & $\begin{array}{c}\text { Booleana } \\
\text { Freqüêncla }\end{array}$ & $\begin{array}{l}5,78 \pm 14,79 \\
5,78 \pm 14,79\end{array}$ & $\begin{array}{l}5,78 \pm 14,79 \\
5,78 \pm 14,75\end{array}$ & $\begin{array}{c}100 \pm 0,00 \\
98,57 \pm 4,52\end{array}$ & $\begin{array}{l}96,37 \pm 9,60 \\
95,60 \pm 9,58\end{array}$ \\
\hline
\end{tabular}

Tabela 6.16: Experimento com DCan_set e algoritmo SVM, com redução para 3.000 features

Observa-se a redução para 3.000 features corresponde a $21,7 \%$ do número total de atributos. Não houve melhora significativa dos resultados, em função do aumento de atributos, houve sim até uma piora nos resultados.

Neste experimento pode ser observado que o erro total foi o mesmo para todas as configurações do SVM quando utilizado a representação por peso booleano. Na atribuição de peso por freqüência do atributo nos documentos, os valores se mantiveram constantes para o Kernel Radial Basis ( $\mathrm{t}=2$ ) com o parâmetro g de 0,8, 1,0 e 1,2.

Neste experimento o menor erro total foi encontrado para várias configurações, sendo esse valor quase que constante para toda a representação booleana, com exceção do SVM com parâmetro $(\mathrm{t}=1 ; \mathrm{d}=2)$. Com relação a cobertura e $F_{1}$ também não pode se tirar grandes conclusões, uma vez que a variação entre os dados foi muito pequena. Com relação ao erro da classe positiva também teve um comportamento semelhante ao erro total, sendo que na maioria dos casos igual ao erro total.

Calculando a diferença em desvios-padrão temos os resultados mostrados na Tabela 6.17 , com a representação booleana e de freqüência. Baseado nas diferenças em desvios- 
padrão do erro total o maior valor encontrado é $\mathbf{0 , 1 8 7 8}$, usando DCan_set com redução para 3.000 features, indicando que o SVM com parâmetros $(t=1 ; d=1)$ e representação booleana possui melhor desempenho quando comparado com SVM com parâmetros $(t=1 ; d=2)$ e representação de freqüência. Esse mesmo valor foi encontrado com outras comparações também.

\begin{tabular}{|c|c|c|c|c|c|c|c|c|}
\hline Parâmetros & $\begin{array}{c}t=1 ; d=1 \\
\text { Bool }\end{array}$ & $\begin{array}{c}t=1 ; d=1 \\
\text { Freq }\end{array}$ & $\begin{array}{c}t=1 ; d=2 \\
\text { Bool }\end{array}$ & $\begin{array}{c}t=1 ; d=2 \\
\text { Freq }\end{array}$ & $\begin{array}{c}\mathrm{t}=2 ; \mathrm{g}=0,6 \\
\text { Bool }\end{array}$ & $\begin{array}{c}t=2 ; g=0,6 \\
\text { Freq }\end{array}$ & $\begin{array}{c}t=2 ; g=0,8 \\
\text { Bool }\end{array}$ & $\begin{array}{c}t=2 ; g=0,8 \\
F_{\text {req }}\end{array}$ \\
\hline $\begin{array}{l}t=1 ; d=1 \\
\text { Bool }\end{array}$ & - & 0,0846 & 0 & 0,1878 & 0 & 0,0965 & 0 & 0 \\
\hline $\begin{array}{l}t=1 ; d=1 \\
\text { Freq }\end{array}$ & & - & $-0,0846$ & 0,1104 & $-0,0846$ & 0,0121 & $-0,0846$ & $-0,0846$ \\
\hline $\begin{array}{l}t=1 ; d=2 \\
\text { Bool }\end{array}$ & & & - & 0,1878 & 0 & 0,0965 & 0 & 0 \\
\hline $\begin{array}{l}t=1 ; d=2 \\
\text { Freq }\end{array}$ & & & & - & $-0,1878$ & $-0,0990$ & $-0,1878$ & $-0,1878$ \\
\hline $\begin{array}{l}t=2 ; g=0,6 \\
\text { Bool }\end{array}$ & & & & & - & 0,0965 & 0 & 0 \\
\hline $\begin{array}{l}t=2 ; g=0,6 \\
\text { Freq }\end{array}$ & & & & & & - & $-0,0965$ & $-0,0965$ \\
\hline $\begin{array}{l}t=2 ; g=0,8 \\
\text { Bool }\end{array}$ & & & & & & & - & 0 \\
\hline
\end{tabular}

Tabela 6.17: Diferença em desvios-padrão com DCan_set e algoritmo SVM, com redução para 3.000 features 


\subsection{Resultados: Experimento 2 - DStem_set}

Os experimentos realizados com a identificação de atributos pelo método de stemming e utilizando-se das definições de pré-processamento baseado em (Mladenić 1998) foram denominados de Experimento 2. Observa-se que o script ScpDStem, gerou o conjunto de dados DStem_set.

Para o kernel polinomial $(t=1)$, testou-se $\mathrm{d}$ com os valores 1 e 2 . Não foi possível utilizar o parâmetro $t \neq 1 \mathrm{com}$ nenhuma redução de feature, o que implicaria utilizar outros kernels que não o polinomial, pois não foi possível executar o algoritmo SVM com tais parâmetros.

\subsubsection{Sem Redução de features}

O número total de features desse experimento é de 15.854, conforme mostrado na Tabela 5.3 do Capítulo 5.

$\mathrm{Na}$ Tabela 6.18 são mostrados os resultados para DStem_set, sem redução de features, com o algoritmo SVM.

\begin{tabular}{|l||c|c|c|c|c|}
\hline \hline Algoritmo & Representaçāo & $\begin{array}{c}\text { erro total } \\
(\%)\end{array}$ & $\begin{array}{c}\text { erro da } \\
\text { classe + (\%) }\end{array}$ & $\begin{array}{c}\text { cobertura } \\
(\%)\end{array}$ & $\begin{array}{c}\mathbf{F}_{\mathbf{1}} \\
(\%)\end{array}$ \\
\hline \hline SVM & Booleana & $7,05 \pm 9,52$ & $5,11 \pm 8,58$ & $90,89 \pm 15,76$ & $92,25 \pm 11,54$ \\
$(\mathrm{t}=1 ; \mathrm{d}=1)$ & Freqüência & $6,96 \pm 8,04$ & $4,36 \pm 7,37$ & $91,07 \pm 15,70$ & $92,28 \pm 10,18$ \\
\hline SVM & Booleana & $21,43 \pm 11,50$ & $12,03 \pm 16,21$ & $66,07 \pm 17,90$ & $74,59 \pm 16,04$ \\
$(\mathrm{t}=1 ; \mathrm{d}=2)$ & Freqüência & $12,95 \pm 11,88$ & $\mathbf{7 , 5 0 \pm 1 0 , 5 4}$ & $82,32 \pm 18,77$ & $86,29 \pm 14,75$ \\
\hline \hline
\end{tabular}

Tabela 6.18: Experimento com DStem_set e algoritmo SVM, sem redução de features

Nos resultados deste experimento pode ser observado que o erro total variou para todas as configurações do SVM, utilizando as duas representações com atribuição de peso booleano e de freqüência.

Neste experimento o menor erro total foi encontrado para a configuração default do $\operatorname{SVM}(t=1 ; d=1)$ e representação por freqüência.

Com relação ao erro da classe positiva também obteve um comportamento semelhante ao erro total, lembrando que nossos dados vieram de uma classe bivalorada, balanceada, como pode ser observado pela Tabela 5.1 do Capítulo 5 . Com relação à cobertura 
e $F_{1}$, neste experimento houve uma alteração significativa, principalmente para SVM com parâmetros $(\mathrm{t}=1 ; \mathrm{d}=2)$.

Calculando a diferença em desvios-padrão temos os resultados mostrados na Tabela 6.19 , com a representação booleana e de freqüência.

\begin{tabular}{|l||c|c|c|c|}
\hline \hline Parâmetros & $\begin{array}{c}\mathrm{t}=1 ; \mathrm{d}=1 \\
\text { Bool }\end{array}$ & $\begin{array}{c}\mathrm{t}=1 ; \mathrm{d}=1 \\
\text { Freq }\end{array}$ & $\begin{array}{c}\mathrm{t}=1 ; \mathrm{d}=2 \\
\text { Bool }\end{array}$ & $\begin{array}{c}\mathrm{t}=1 ; \mathrm{d}=2 \\
\text { Freq }\end{array}$ \\
\hline $\begin{array}{l}\mathrm{t}=1 ; \mathrm{d}=1 \\
\text { Bool }\end{array}$ & - & $-0,0102$ & 1,3622 & 0,5481 \\
\hline $\begin{array}{l}\mathrm{t}=1 ; \mathrm{d}=1 \\
\text { Freq }\end{array}$ & & - & 1,4584 & 0,5905 \\
\hline $\begin{array}{l}\mathrm{t}=1 ; \mathrm{d}=2 \\
\text { Bool }\end{array}$ & & & - & $-0,7253$ \\
\hline \hline
\end{tabular}

Tabela 6.19: Diferença em desvios-padrão com DStem_set e algoritmo SVM, sem redução de features

Baseado nas diferenças em desvios-padrão do erro total, o maior valor encontrado é 1,4584, usando DStem_set sem redução de features, ou seja, o SVM com parâmetros $(t=1 ; d=1)$ e representação de freqüência possui desempenho superior ao SVM com parâmetros $(t=1 ; d=2)$ e representação booleana.

\subsubsection{Redução para 250 features}

Na Tabela 6.20 são mostrados os resultados para DStem_set e redução para 250 features, com o algoritmo SVM e kernel polinomial.

\begin{tabular}{|l||c|c|c|c|c|}
\hline \hline Algoritmo & Representação & $\begin{array}{c}\text { erro total } \\
(\%)\end{array}$ & $\begin{array}{c}\text { erro da } \\
\text { classe }+(\%)\end{array}$ & $\begin{array}{c}\text { cobertura } \\
(\%)\end{array}$ & $\begin{array}{c}F_{1} \\
(\%)\end{array}$ \\
\hline \hline SVM & Booleana & $7,85 \pm 9,32$ & $6,36 \pm 8,66$ & $88,04 \pm 16,50$ & $92,25 \pm 11,54$ \\
$(\mathrm{t}=1 ; \mathrm{d}=1)$ & Freqüêncla & $6,34 \pm 9,78$ & $3,47 \pm 5,60$ & $91,07 \pm 19,63$ & $92,80 \pm 10,12$ \\
\hline SVM & Booleana & $7,05 \pm 7,48$ & $2,36 \pm 4,99$ & $88,39 \pm 15,02$ & $91,96 \pm 9,79$ \\
$(\mathrm{t}=1 ; \mathrm{d}=2)$ & Freqüuência & $12,77 \pm 11,37$ & $10,22 \pm 11,96$ & $84,82 \pm 18,43$ & $86,16 \pm 14,36$ \\
\hline \hline
\end{tabular}

Tabela 6.20: Experimento com DStem_set e algoritmo SVM, com redução para 250 features

A redução para 250 features corresponde a $1,6 \%$ do número total de atributos. Houve uma pequena melhora dos resultados com parâmetros $(t=1 ; d=1)$ e representação 
por freqüência e piora na representação boolena, quando comparado com os resultados obtidos sem nenhuma redução de features.

Nos resultados deste experimento pode ser observado que o erro total variou para todas as configurações do SVM, utilizando as duas representações com atribuição de peso booleano e de freqüência.

Neste experimento o menor erro total foi encontrado para a configuração default do SVM $(t=1 ; d=1)$ e representação por freqüência.

O erro da classe positiva obteve um comportamento semelhante ao erro total, lembrando que nossos dados vieram de uma classe bivalorada, balanceada, como pode ser observado pela Tabela 5.1 do Capítulo 5. Com relação a cobertura e $F_{1}$ neste experimento houve alteração, menos significativa quando comparada com o experimento sem redução, principalmente para SVM com parâmetros $(\mathrm{t}=1 ; \mathrm{d}=2)$.

Baseado nas diferenças em desvios-padrão do erro total mostrado na Tabela 6.21, o maior valor encontrado é $\mathbf{0 , 6 0 6 3}$, usando DStem_set com redução para 250 features. Isso indica que o SVM com parâmetros $(\mathrm{t}=1 ; \mathrm{d}=1)$ e representação de freqüência possui desempenho levemente melhor que o SVM com parâmetro $(t=1 ; d=2)$ e representação de freqüência.

\begin{tabular}{|l||c|c|c|c|}
\hline \hline Parâmetros & $\begin{array}{c}t=1 ; d=1 \\
\text { Bool }\end{array}$ & $\begin{array}{c}t=1 ; d=1 \\
\text { Freq }\end{array}$ & $\begin{array}{c}t=1 ; d=2 \\
\text { Bool }\end{array}$ & $\begin{array}{c}t=1 ; d=2 \\
\text { Freq }\end{array}$ \\
\hline $\begin{array}{l}t=1 ; d=1 \\
\text { Bool }\end{array}$ & - & $-0,1581$ & $-0,0947$ & 0,4733 \\
\hline $\begin{array}{l}t=1 ; d=1 \\
\text { Freq }\end{array}$ & & - & 0,0816 & 0,6063 \\
$\begin{array}{l}t=1 ; d=2 \\
\text { Bool }\end{array}$ & & & - & 0,5944 \\
\hline \hline
\end{tabular}

Tabela 6.21: Diferença em desvios-padrão com DStem_set e algoritmo SVM, com redução para 250 features

\subsubsection{Redução para 500 features}

Aplicou-se o script de redução para utilização do algoritmo SVM e redução para 500 features, utilizando-se DStem_set com kernel polinomial. 
Na Tabela 6.22 são mostrados os resultados para DStem_set, com redução para 500 features.

\begin{tabular}{|l||c|c|c|c|c|}
\hline \hline Algoritmo & Representaçāo & $\begin{array}{c}\text { erro total } \\
(\%)\end{array}$ & $\begin{array}{c}\text { erro da } \\
\text { classe + (\%) }\end{array}$ & $\begin{array}{c}\text { cobertura } \\
(\%)\end{array}$ & $\begin{array}{c}\mathbf{F}_{\mathbf{1}} \\
\mathbf{( \% )}\end{array}$ \\
\hline \hline SVM & Booleana & $7,14 \pm 9,65$ & $4,22 \pm 7,22$ & $89,46 \pm 16,89$ & $92,25 \pm 11,54$ \\
$(\mathrm{t}=1 ; \mathrm{d}=1)$ & Freqüência & $6,96 \pm 9,97$ & $4,36 \pm 7,37$ & $91,07 \pm 19,63$ & $92,28 \pm 10,18$ \\
\hline SVM & Booleana & $8,39 \pm 7,18$ & $3,25 \pm 7,08$ & $86,96 \pm 14,46$ & $90,66 \pm 9,34$ \\
$(\mathrm{t}=1 ; \mathrm{d}=2)$ & Freqüência & $10,80 \pm 10,97$ & $8,47 \pm 9,83$ & $84,82 \pm 18,43$ & $87,20 \pm 14,29$ \\
\hline
\end{tabular}

Tabela 6.22: Experimento com DStem_set e algoritmo SVM, com redução para 500 features

A redução para 500 features corresponde a $3,2 \%$ do número total de atributos. Houve uma pequena melhora dos resultados, quando comparado com os resultados obtidos com redução para 250 features e representação booleana para os parêmtros $(t=1 ; d=1)$. Ocorreu uma pequena piora dos resultados, quando comparado com os resultados obtidos com reduçāo para 250 features e representação por freqüência para os parâmetros $(t=1 ; d=1)$.

Nos resultados deste experimento pode ser observado que o erro total variou para todas as configurações do SVM, utilizando as duas representações com atribuição de peso booleano e de freqüência.

Neste experimento o menor erro total foi encontrado para a configuração default do SVM $(t=1 ; d=1)$ e representação por freqüência.

O erro da classe positiva obteve um comportamento semelhante ao erro total. Com relação à cobertura e $F_{1}$ neste experimento houve alteração, principalmente para SVM com parâmetros $(\mathrm{t}=1 ; \mathrm{d}=2)$.

Baseado nas diferenças em desvios-padrão do erro total mostrado na Tabela 6.23, o maior valor encontrado é 0,3663 . Isso indica que o SVM com parâmetros $(t=1 ; d=1)$ e representação de freqüência possui desempenho ligeiramente melhor quando comparado com SVM com parâmetros $(t=1 ; d=2)$ e representação de freqüênicia. 


\begin{tabular}{|l||c|c|c|c|}
\hline \hline Parâmetros & $\begin{array}{c}t=1 ; d=1 \\
\text { Bool }\end{array}$ & $\begin{array}{c}t=1 ; d=1 \\
\text { Freq }\end{array}$ & $\begin{array}{c}t=1 ; d=2 \\
\text { Bool }\end{array}$ & $\begin{array}{c}t=1 ; d=2 \\
\text { Freq }\end{array}$ \\
\hline $\begin{array}{l}t=1 ; d=1 \\
\text { Bool }\end{array}$ & - & $-0,0183$ & 0,1470 & 0,3543 \\
\hline $\begin{array}{l}t=1 ; d=1 \\
\text { Freq }\end{array}$ & & - & 0,1646 & 0,3663 \\
\hline $\begin{array}{l}t=1 ; d=2 \\
\text { Bool }\end{array}$ & & & - & 0,2600 \\
\hline \hline
\end{tabular}

Tabela 6.23: Diferença em desvios-padrão com DStem_set e algoritmo SVM, com redução para 500 features

\subsubsection{Redução para 750 features}

Aplicou-se o script de redução para utilização do algoritmo SVM e redução para 750 features, utilizando-se DStem_set com kernel polinomial.

$\mathrm{Na}$ Tabela 6.24 são mostrados os resultados desse experimento, utilizando SVM, para representação booleana e freqüência.

\begin{tabular}{|c|c|c|c|c|c|}
\hline Algoritmo & Representaçäo & $\begin{array}{c}\text { erro total } \\
(\%)\end{array}$ & $\begin{array}{c}\text { erro da } \\
\text { classe }+(\%)\end{array}$ & $\begin{array}{c}\text { cobertura } \\
(\%)\end{array}$ & $\begin{array}{c}F_{1} \\
(\%)\end{array}$ \\
\hline $\begin{array}{l}\text { SVM } \\
(t=1 ; d=1)\end{array}$ & $\begin{array}{c}\text { Booleana } \\
\text { Frequiêncla }\end{array}$ & $\begin{array}{l}7,14 \pm 9,65 \\
6,96 \pm 8,04\end{array}$ & $\begin{array}{l}4,22 \pm 7,22 \\
4,36 \pm 7,37\end{array}$ & $\begin{array}{l}89,46 \pm 16,89 \\
91,07 \pm 15,70\end{array}$ & $\begin{array}{l}92,25 \pm 11,54 \\
92,28 \pm 10,18\end{array}$ \\
\hline $\begin{array}{l}\text { SVM } \\
(t=1 ; d=2)\end{array}$ & $\begin{array}{c}\text { Booleana } \\
\text { Frequiência }\end{array}$ & $\begin{array}{c}7,68 \pm 7,66 \\
10,09 \pm 11,46\end{array}$ & $\begin{array}{c}3,25 \pm 7,08 \\
5,97 \pm 10,10\end{array}$ & $\begin{array}{l}88,39 \pm 15,02 \\
84,82 \pm 18,43\end{array}$ & $\begin{array}{r}91,43 \pm 9,80 \\
88,45 \pm 14,86\end{array}$ \\
\hline
\end{tabular}

Tabela 6.24: Experimento com DStem_set e algoritmo SVM, com redução para 750 features

A redução para 750 features corresponde a $4,7 \%$ do número total de atributos. Houve uma pequena melhora, quando comparado com os resultados obtidos com redução para 500 features.

Nos resultados deste experimento pode ser observado que o erro total variou para todas as configurações do SVM, utilizando as duas representações com atribuição de peso booleano e de freqüência, ficando exatamente igual aos resultados obtidos para SVM com parâmetros $(t=1 ; d=1)$ para as duas representações, em relação a redução para 500 features.

Neste experimento o menor erro total foi encontrado para a configuração default 
do SVM $(t=1 ; d=1)$ e representação por freqüência, semelhante ao obtido com a redução para 500 features.

O erro da classe positiva obteve um comportamento semelhante ao erro total. Com relação à cobertura e $F_{1}$, neste experimento houve alteração, menos significativa quando comparada com o experimento sem redução, principalmente para SVM com parâmetros $(\mathrm{t}=1 ; \mathrm{d}=2)$.

Baseado nas diferenças em desvios-padrão do erro total mostrados na Tabela 6.25, o maior valor encontrado é $\mathbf{0 , 3 1 6 2}$. Isso indica que o SVM com parâmetros $(t=1 ; d=1)$ e representação de freqüência possui desempenho ligeiramente melhor quando comparado com SVM com parâmetros $(t=1 ; d=2)$ e representaçāo de freqüência.

\begin{tabular}{|l||c|c|c|c|}
\hline \hline Parametros & $\begin{array}{c}t=1 ; d=1 \\
\text { Bool }\end{array}$ & $\begin{array}{c}t=1 ; d=1 \\
\text { Freq }\end{array}$ & $\begin{array}{c}t=1 ; d=2 \\
\text { Bool }\end{array}$ & $\begin{array}{c}t=1 ; d=2 \\
\text { Freq }\end{array}$ \\
\hline \hline $\begin{array}{l}t=1 ; d=1 \\
\text { Bool }\end{array}$ & - & $-0,0203$ & 0,0620 & 0,2785 \\
\hline $\begin{array}{l}t=1 ; d=1 \\
\text { Freq }\end{array}$ & & - & 0,0917 & 0,3162 \\
\hline $\begin{array}{l}t=1 ; d=2 \\
\text { Bool }\end{array}$ & & & - & 0,2473 \\
\hline \hline
\end{tabular}

Tabela 6.25: Diferença em desvios-padrāo com DStem_set e algoritmo SVM, com redução para 750 features

\subsubsection{Redução para 1.000 features}

Aplicou-se o script de redução para utilização do algoritmo SVM e redução para 1.000 features, utilizando-se DStem_set com kernel polinomial.

$\mathrm{Na}$ Tabela 6.26 são mostrados os resultados desse experimento, utilizando SVM, para representação booleana e freqüência.

A redução para 1.000 features corresponde a $6,3 \%$ do número total de atributos. Houve uma pequena melhora em relação ao erro total para parâmetros $(t=1 ; d=1)$ em ambas as representações e piora com os parâmetros $(\mathrm{t}=1 ; \mathrm{d}=2)$ também para ambas as representações, quando comparado com os resultados obtidos com redução para 750 features. 


\begin{tabular}{|l||c|c|c|c|c|}
\hline \hline Algoritmo & Representação & $\begin{array}{c}\text { erro total } \\
(\%)\end{array}$ & $\begin{array}{c}\text { erro da } \\
\text { classe }+(\%)\end{array}$ & $\begin{array}{c}\text { cobertura } \\
(\%)\end{array}$ & $\begin{array}{c}\text { F }_{\mathbf{1}} \\
(\%)\end{array}$ \\
\hline \hline SVM & Booleana & $7,14 \pm 9,65$ & $4,22 \pm 7,22$ & $89,46 \pm 16,89$ & $92,25 \pm 11,54$ \\
$(t=1 ; d=1)$ & Freqüêncla & $6,34 \pm 7,80$ & $4,22 \pm 7,22$ & $92,32 \pm 15,88$ & $92,35 \pm 10,15$ \\
\hline SVM & Booleana & $8,30 \pm 7,21$ & $3,25 \pm 7,08$ & $87,14 \pm 14,45$ & $90,77 \pm 9,37$ \\
$(t=1 ; d=2)$ & Freqüência & $10,18 \pm 9,34$ & $6,72 \pm 9,16$ & $86,07 \pm 14,96$ & $88,94 \pm 11,16$ \\
\hline \hline
\end{tabular}

Tabela 6.26: Experimento com DStem_set e algoritmo SVM, com redução para 1.000 features

Nos resultados deste experimento pode ser observado que o erro total variou para todas as configurações do SVM, utilizando a representação com atribuição de peso booleano e de freqüência. Os resultados obtidos para SVM com parâmetros $(t=1 ; d=1)$ para a representação booleana foi exatamente igual e a representação de freqüência ocorreu uma pequena melhora, comparando com redução para 500 features.

Neste experimento o menor erro total foi encontrado para a configuração default do $\operatorname{SVM}(t=1 ; d=1)$ e representação por freqüência, semelhante ao obtido com a redução para sem redução, 250,500 e 750 features.

O erro da classe positiva obteve um comportamento semelhante ao erro total. Com relação à cobertura e $F_{1}$, neste experimento houve alteração, menos significativa quando comparada com o experimento sem redução, principalmente para SVM com parâmetros $(\mathrm{t}=1 ; \mathrm{d}=2)$.

Baseado nas diferenças em desvios-padrão do erro total mostrados na Tabela 6.27, o maior valor encontrado é 0,4463 . Isso indica que o $S V M$ com parâmetros $(t=1 ; d=1)$ e representação de freqüência possui melhor desempenho quando comparado com SVM com parâmetros $(\mathrm{t}=1 ; \mathrm{d}=2)$ e representação de freqüência.

\subsubsection{Redução para 1.500 features}

Aplicou-se o script de redução para utilização do algoritmo SVM e redução para 1.500 features, utilizando-se DStem_set com kernel polinomial.

$\mathrm{Na}$ Tabela 6.28 são mostrados os resultados desse experimento, utilizando SVM, para representação booleana e freqüência.

A redução para 1.500 features corresponde a $9,5 \%$ do número total de atributos. 


\begin{tabular}{|l||c|c|c|c|}
\hline \hline Parâmetros & $\begin{array}{c}t=1 ; d=1 \\
\text { Bool }\end{array}$ & $\begin{array}{c}t=1 ; d=1 \\
\text { Freq }\end{array}$ & $\begin{array}{c}t=1 ; d=2 \\
\text { Bool }\end{array}$ & $\begin{array}{c}t=1 ; d=2 \\
\text { Freq }\end{array}$ \\
\hline $\begin{array}{l}t=1 ; d=1 \\
\text { Bool }\end{array}$ & - & $-0,0912$ & 0,1362 & 0,3201 \\
\hline $\begin{array}{l}t=1 ; d=1 \\
\text { Freq }\end{array}$ & & - & 0,2610 & 0,4463 \\
\hline $\begin{array}{l}t=1 ; d=2 \\
\text { Bool }\end{array}$ & & & - & 0,2253 \\
\hline \hline
\end{tabular}

Tabela 6.27: Diferença em desvios-padrão com DStem_set e algoritmo SVM, com redução para 1.000 features

\begin{tabular}{|l||c|c|c|c|c|}
\hline \hline Algoritmo & Representaçāo & $\begin{array}{c}\text { erro total } \\
(\%)\end{array}$ & $\begin{array}{c}\text { erro da } \\
\text { classe }+(\%)\end{array}$ & $\begin{array}{c}\text { cobertura } \\
(\%)\end{array}$ & $\begin{array}{c}F_{\mathbf{1}} \\
\mathbf{( \% )}\end{array}$ \\
\hline \hline SVM & Booleana & $6,43 \pm 9,32$ & $4,22 \pm 7,22$ & $90,89 \pm 15,76$ & $92,25 \pm 11,54$ \\
$(\mathrm{t}=1 ; \mathrm{d}=1)$ & Freqüêncla & $6,96 \pm 8,04$ & $4,36 \pm 7,37$ & $91,07 \pm 15,70$ & $92,28 \pm 10,18$ \\
\hline SVM & Booleana & $9,55 \pm 7,86$ & $3,46 \pm 7,65$ & $84,64 \pm 14,14$ & $89,36 \pm 9,75$ \\
$(\mathrm{t}=\mathbf{1} ; \mathrm{d}=2)$ & Freqùèncla & $10,09 \pm 11,46$ & $5,97 \pm 10,10$ & $84,82 \pm 18,43$ & $88,45 \pm 14,86$ \\
\hline \hline
\end{tabular}

Tabela 6.28: Experimento com DStem_set e algoritmo SVM, com redução para 1.500 features

Ocorreu uma pequena melhora em relação ao erro total, quando comparado com os resultados obtidos com redução para 1.000 features para representação booleana e uma pequena piora para representação de freqüência, ambos com os parâmetros do SVM $(t=1 ; d=1)$.

Nos resultados deste experimento pode ser observado que o erro total variou para todas as configurações do SVM, utilizando a representação com atribuição de peso booleano e de freqüência.

Neste experimento o menor erro total foi encontrado para a configuração default do $\operatorname{SVM}(t=1 ; d=1)$ e representação booleana, ao contrário do que ocorreu com as reduções para $250,500,750$ e 1000 features e sem redução, onde os melhores resultados foi obtido pela representação de freqüência.

O erro da classe positiva obteve um comportamento semelhante ao erro total. Com relação à cobertura e $F_{1}$, neste experimento houve alteração menos significativa quando comparada com o experimento sem redução, principalmente para SVM com parâmetros $(\mathrm{t}=1 ; \mathrm{d}=2)$.

Baseado nas diferenças em desvios-padrão do erro total mostrados na Tabela 6.29, 
o maior valor encontrado é $\mathbf{0 , 3 7 2 3}$. Isso indica que o SVM com parâmetros $(t=1 ; d=1)$ e representação booleana possui melhor desempenho quando comparado com SVM com parâmetros $(t=1 ; d=2)$ e representação booleana.

\begin{tabular}{|l||c|c|c|c|}
\hline \hline Parâmetros & $\begin{array}{c}\mathrm{t}=1 ; \mathrm{d}=1 \\
\text { Bool }\end{array}$ & $\begin{array}{c}\mathrm{t}=1 ; \mathrm{d}=1 \\
\text { Freq }\end{array}$ & $\begin{array}{c}\mathrm{t}=1 ; \mathrm{d}=2 \\
\text { Bool }\end{array}$ & $\begin{array}{c}\mathrm{t}=1 ; \mathrm{d}=2 \\
\text { Freq }\end{array}$ \\
\hline \hline $\begin{array}{l}\mathrm{t}=1 ; \mathrm{d}=1 \\
\text { Bool }\end{array}$ & - & 0,0712 & $\mathbf{0 , 3 7 2 3}$ & 0,3590 \\
\hline $\begin{array}{l}\mathrm{t}=1 ; \mathrm{d}=1 \\
\text { Freq }\end{array}$ & & - & 0,3258 & 0,3162 \\
\hline $\begin{array}{l}\mathrm{t}=1 ; \mathrm{d}=2 \\
\text { Bool }\end{array}$ & & & - & 0,0550 \\
\hline \hline
\end{tabular}

Tabela 6.29: Diferença em desvios-padrão com DStem_set e algoritmo SVM, com redução para 1.500 features

\subsubsection{Redução para 3.000 features}

Aplicou-se o script de redução para utilização do algoritmo SVM e redução para 3.000 features, utilizando-se DStem_set com kernel polinomial.

Na Tabela 6.30 são mostrados os resultados desse experimento, utilizando SVM, para representação booleana e freqüência.

\begin{tabular}{|l||c|c|c|c|c|}
\hline \hline Algoritmo & Representaçāo & $\begin{array}{c}\text { erro total } \\
(\%)\end{array}$ & $\begin{array}{c}\text { erro da } \\
\text { classe }+(\%)\end{array}$ & $\begin{array}{c}\text { cobertura } \\
(\%)\end{array}$ & $\begin{array}{c}F_{1} \\
(\%)\end{array}$ \\
\hline \hline SVM & Booleana & $6,43 \pm 9,32$ & $4,22 \pm 7,22$ & $90,89 \pm 15,76$ & $92,25 \pm 11,54$ \\
$(\mathrm{t}=1 ; \mathrm{d}=1)$ & Freqüencta & $6,96 \pm 8,04$ & $4,36 \pm 7,37$ & $91,07 \pm 15,70$ & $92,28 \pm 10,18$ \\
\hline SVM & Booleana & $11,52 \pm 8,58$ & $3,46 \pm 7,65$ & $80,71 \pm 16,73$ & $86,61 \pm 12,34$ \\
$(\mathrm{t}=1 ; \mathrm{d}=2)$ & Frequilencla & $11,43 \pm 11,63$ & $\mathbf{7 , 5 0 \pm 1 0 , 5 4}$ & $83,07 \pm 20,04$ & $86,59 \pm 16,21$ \\
\hline \hline
\end{tabular}

Tabela 6.30: Experimento com DStem_set e algoritmo SVM, com redução para 3.000 features

A redução para 3.000 features corresponde a 18,9\% do número total de atributos. Não ocorreu nenhuma modificação em relação ao erro total com parâmetros do SVM $(\mathrm{t}=1 ; \mathrm{d}=1)$ e representaçāo de freqüuência e uma ligeira piora com parâmetros do SVM $(t=1 ; d=2)$ em ambas as representações, quando comparado com os resultados obtidos com redução para 1.500 features. 
Nos resultados deste experimento pode ser observado que o erro total variou para todas as configurações do SVM, utilizando a representação com atribuição de peso booleano e de freqüência.

Neste experimento o menor erro total foi encontrado para a configuração default do SVM $(\mathrm{t}=1 ; \mathrm{d}=1)$ e representação booleana, semelhante ao ocorrido com a redução para 1.500 features.

O erro da classe positiva obteve um comportamento semelhante ao erro total. Com relação à cobertura e $F_{1}$, neste experimento houve alteração, menos significativa quando comparada com o experimento sem redução, principalmente para SVM com parâmetros $(\mathrm{t}=1 ; \mathrm{d}=2)$.

Baseado nas diferenças em desvios-padrão do erro total mostrados na Tabela 6.31, o melhor valor encontrado é $\mathbf{0 , 5 7 8 3}$. Isso indica que o SVM com parâmetros $(t=1 ; d=1)$ e representação booleana possui desempenho ligeiramente melhor quando comparado com SVM com parâmetros $(t=1 ; d=2)$ e representação booleana.

\begin{tabular}{|l||c|c|c|c|}
\hline \hline Parâmetros & $\begin{array}{c}t=1 ; d=1 \\
\text { Bool }\end{array}$ & $\begin{array}{c}t=1 ; d=1 \\
\text { Freq }\end{array}$ & $\begin{array}{c}t=1 ; d=2 \\
\text { Bool }\end{array}$ & $\begin{array}{c}t=1 ; d=2 \\
\text { Freq }\end{array}$ \\
\hline $\begin{array}{l}t=1 ; d=1 \\
\text { Bool }\end{array}$ & - & 0,0712 & 0,5783 & 0,4830 \\
\hline $\begin{array}{l}t=1 ; d=1 \\
\text { Freq }\end{array}$ & & - & 0,5484 & 0,4471 \\
\hline $\begin{array}{l}t=1 ; d=2 \\
\text { Bool }\end{array}$ & & & - & $-0,0088$ \\
\hline \hline
\end{tabular}

Tabela 6.31: Diferença em desvios-padrão com DStem_set e algoritmo SVM, com redução para 3.000 features 


\subsection{Resultados: Experimento 3 - NCan_set}

Os experimentos realizados com a identificação de atributos pelo método canônico e utilizando-se das definições de Pré-processamento baseado em (Weiss \& Indurkhya 1998) foram denominados de Experimento 3. O script ScpNCan, gerou o conjunto de dados utilizando o conjunto de dados NCan_set.

Para o kernel polinomial $(\mathrm{t}=1)$, testou-se $\mathbf{d}$ com os valores 1 e 2 . Não foi possível utilizar o parâmetro $t \neq 1$ com nenhuma redução de feature, o que implica em utilizar outros kernels que não o polinomial, pois não foi possível executar o algoritmo SVM com tais parâmetros.

\subsubsection{Sem Redução de features}

O número total de features desse experimento é de 2.068 , conforme mostrado na Tabela 5.3 do Capítulo 5.

$\mathrm{Na}$ Tabela 6.32 são mostrados os resultados para NCan_set, sem redução de features, com o algoritmo SVM.

\begin{tabular}{|l||c|c|c|c|c|}
\hline \hline Algoritmo & Representaçāo & $\begin{array}{c}\text { erro total } \\
(\%)\end{array}$ & $\begin{array}{c}\text { erro da } \\
\text { classe + (\%) }\end{array}$ & $\begin{array}{c}\text { cobertura } \\
(\%)\end{array}$ & $\begin{array}{c}\mathrm{F}_{1} \\
(\%)\end{array}$ \\
\hline \hline SVM & Booleana & $7,14 \pm 8,51$ & $6,25 \pm 8,61$ & $94,64 \pm 9,67$ & $92,98 \pm 8,60$ \\
$(\mathrm{t}=1 ; \mathrm{d}=1)$ & Frequiuencia & $3,30 \pm 4,57$ & $1,25 \pm 3,95$ & $94,82 \pm 8,93$ & $96,47 \pm 5,03$ \\
\hline $\mathrm{SVM}$ & Booleana & $25,89 \pm 29,04$ & $14,21 \pm 9,93$ & $79,82 \pm 25,46$ & $81,36 \pm 19,28$ \\
$(\mathrm{t}=1 ; \mathrm{d}=2)$ & Freqüùncia & $16,16 \pm 12,25$ & $12,31 \pm 14,65$ & $83,93 \pm 19,08$ & $83,42 \pm 12,41$ \\
\hline
\end{tabular}

Tabela 6.32: Experimento com NCan_set e algoritmo SVM, sem redução de features

Nos resultados deste experimento pode ser observado que o erro total variou para todas as configuraçōes do SVM, utilizando as duas representação com atribuição de peso booleano e de freqüência.

Neste experimento o menor erro total foi encontrado para a configuração default do $\operatorname{SVM}(t=1 ; d=1)$ e representação por freqüência.

O erro da classe positiva obteve um comportamento semelhante ao erro total. Com relação à cobertura e $F_{1}$, neste experimento houve uma alteração significativa, principalmente para SVM com parâmetros $(\mathrm{t}=1 ; \mathrm{d}=2)$. 
Baseado nas diferenças em desvios-padrão do erro total mostrados na Tabela 6.33, o maior valor encontrado é 1,3910 . Isso indica que o SVM com parâmetros $(t=1 ; d=1)$ e representação de freqüência possui melhor desempenho quando comparado com SVM com parâmetros $(t=1 ; d=2)$ e representação de freqüência.

\begin{tabular}{|l||c|c|c|c|}
\hline \hline Parâmetros & $\begin{array}{c}t=1 ; d=1 \\
\text { Bool }\end{array}$ & $\begin{array}{c}t=1 ; d=1 \\
\text { Freq }\end{array}$ & $\begin{array}{c}t=1 ; d=2 \\
\text { Bool }\end{array}$ & $\begin{array}{c}t=1 ; d=2 \\
\text { Freq }\end{array}$ \\
\hline $\begin{array}{l}t=1 ; d=1 \\
\text { Bool }\end{array}$ & - & $-0,5622$ & 0,8763 & 0,8552 \\
\hline $\begin{array}{l}t=1 ; d=1 \\
\text { Freq }\end{array}$ & & - & 1,0867 & 1,3910 \\
\hline $\begin{array}{l}t=1 ; d=2 \\
\text { Bool }\end{array}$ & & & - & $-0,4366$ \\
\hline \hline
\end{tabular}

Tabela 6.33: Diferença em desvios-padrão com NCan_set e algoritmo SVM, sem redução de features

\subsubsection{Redução para 250 features}

Na Tabela 6.34 são mostrados os resultados para NCan_set e redução para 250 features, com o algoritmo SVM e kernel polinomial.

\begin{tabular}{|l||c|c|c|c|c|}
\hline \hline Algorltmo & Representaçāo & $\begin{array}{c}\text { orro total } \\
(\%)\end{array}$ & $\begin{array}{c}\text { orro da } \\
\text { classe }+(\%)\end{array}$ & $\begin{array}{c}\text { cobertura } \\
(\%)\end{array}$ & $\begin{array}{c}F_{1} \\
(\%)\end{array}$ \\
\hline \hline SVM & Booleana & $10,27 \pm 8,78$ & $10,95 \pm 8,12$ & $90,89 \pm 13,77$ & $89,52 \pm 9,69$ \\
$(\mathrm{t}=1 ; \mathrm{d}=1)$ & Freqtiêncla & $14,20 \pm 13,27$ & $10,52 \pm 13,83$ & $82,14 \pm 22,32$ & $84,06 \pm 16,50$ \\
\hline SVM & Booleana & $25,98 \pm 29,83$ & $15,93 \pm 15,56$ & $79,82 \pm 25,46$ & $81,24 \pm 21,15$ \\
$(\mathrm{t}=1 ; \mathrm{d}=2)$ & Freqüencia & $18,84 \pm 12,19$ & $14,45 \pm 14,77$ & $78,04 \pm 23,90$ & $78,89 \pm 17,35$ \\
\hline \hline
\end{tabular}

Tabela 6.34: Experimento com NCan_set e algoritmo SVM, com redução para 250 features

A redução para 250 features corresponde a $12,1 \%$ do número total de atributos. Houve piora significativa dos resultados em ambas as representações, quando comparado com os resultados obtidos sem nenhuma redução de features.

Nos resultados deste experimento pode ser observado que o erro total variou para todas as configurações do SVM, utilizando as duas representação com atribuição de peso booleano e de freqüência. 
Neste experimento o menor erro total foi encontrado para a configuração default do SVM $(t=1 ; d=1)$ e representação booleana.

O erro da classe positiva obteve um comportamento semelhante ao erro total. Com relação à cobertura e $F_{1}$, neste experimento houve alterações significativas, principalmente para SVM com parâmetros $(\mathrm{t}=1 ; \mathrm{d}=2)$.

Baseado nas diferenças em desvios-padrāo do erro total mostrados na Tabela 6.35, o maior valor encontrado é $\mathbf{0 , 8 0 6 8}$. Isso indica que o SVM com parâmetros $(t=1 ; d=1)$ e representação booleana possui melhor desempenho quando comparado com SVM com parâmetros $(t=1 ; d=2)$ e representação de freqüência.

\begin{tabular}{|l||c|c|c|c|}
\hline \hline Parâmetros & $\begin{array}{c}t=1 ; \mathrm{d}=1 \\
\text { Bool }\end{array}$ & $\begin{array}{c}t=1 ; \mathrm{d}=1 \\
\text { Freq }\end{array}$ & $\begin{array}{c}\mathrm{t}=1 ; \mathrm{d}=2 \\
\text { Bool }\end{array}$ & $\begin{array}{c}t=1 ; \mathrm{d}=2 \\
\text { Freq }\end{array}$ \\
\hline \hline $\begin{array}{l}\mathrm{t}=1 ; \mathrm{d}=1 \\
\text { Bool }\end{array}$ & - & 0,3493 & 0,7104 & $\mathbf{0 , 8 0 6 8}$ \\
\hline $\begin{array}{l}\mathrm{t}=1 ; \mathrm{d}=1 \\
\text { Freq }\end{array}$ & & - & 0,5064 & 0,3642 \\
\hline $\begin{array}{l}\mathrm{t}=1 ; \mathrm{d}=2 \\
\text { Bool }\end{array}$ & & & - & $-0,3094$ \\
\hline \hline
\end{tabular}

Tabela 6.35: Diferença em desvios-padrão com NCan_set e algoritmo SVM, com redução para 250 features

\subsubsection{Redução para 500 features}

Aplicou-se o script de redução para utilização do algoritmo SVM e redução para 500 features, utilizando-se NCan_set com kernel polinomial.

$\mathrm{Na}$ Tabela 6.36 são mostrados os resultados para NCan_set, com reduçāo para 500 features.

A redução para 500 features corresponde a $24,2 \%$ do número total de atributos. Houve melhora dos resultados, quando comparado com os resultados obtidos com redução para 250 features e representação booleana. O resultado para o erro total se manteve igual ao obtido com redução para 250 features com a representação por freqüência.

Nos resultados deste experimento pode ser observado que o erro total variou para todas as configurações do SVM, utilizando as duas representações com atribuição de peso booleano e de freqüência. 


\begin{tabular}{|l||c|c|c|c|c|}
\hline \hline Algoritmo & Representação & $\begin{array}{c}\text { erro total } \\
(\%)\end{array}$ & $\begin{array}{c}\text { erro da } \\
\text { classe }+(\%)\end{array}$ & $\begin{array}{c}\text { cobertura } \\
(\%)\end{array}$ & $\begin{array}{c}F_{1} \\
(\%)\end{array}$ \\
\hline \hline SVM & Booleana & $7,14 \pm 7,99$ & $7,11 \pm 9,19$ & $94,64 \pm 9,67$ & $92,97 \pm 8,08$ \\
$(t=1 ; d=1)$ & Freqüêncla & $14,02 \pm 13,28$ & $11,87 \pm 14,00$ & $85,00 \pm 19,66$ & $85,13 \pm 14,99$ \\
\hline SVM & Booleana & $25,89 \pm 29,92$ & $14,50 \pm 12,69$ & $79,82 \pm 25,46$ & $81,53 \pm 20,53$ \\
$(t=1 ; d=2)$ & Freqüência & $19,64 \pm 17,01$ & $19,11 \pm 17,35$ & $79,29 \pm 25,45$ & $78,69 \pm 20,72$ \\
\hline \hline
\end{tabular}

Tabela 6.36: Experimento com NCan_set e algoritmo SVM, com redução para 500 features

Neste experimento o menor erro total foi encontrado para a configuração default do SVM $(\mathrm{t}=1 ; \mathrm{d}=1)$ e representação booleana.

O erro da classe positiva obteve um comportamento semelhante ao erro total. Com relação à cobertura e $F_{1}$, neste experimento houve alterações significativas, principalmente para SVM com parâmetros $(\mathrm{t}=1 ; \mathrm{d}=2)$.

Baseado nas diferenças em desvios-padrão do erro total mostrados na Tabela 6.37, o maior valor encontrado é 0,9406 . Isso indica que o SVM com parâmetros $(t=1 ; d=1)$ e representação booleana possui melhor desempenho quando comparado com SVM com parâmetros $(t=1 ; d=2)$ e representação de freqüência.

\begin{tabular}{|l||c|c|c|c|}
\hline \hline Parâmetros & $\begin{array}{c}\mathrm{t}=1 ; \mathrm{d}=1 \\
\text { Bool }\end{array}$ & $\begin{array}{c}\mathrm{t}=1 ; \mathrm{d}=1 \\
\text { Freq }\end{array}$ & $\begin{array}{c}\mathrm{t}=1 ; \mathrm{d}=2 \\
\text { Bool }\end{array}$ & $\begin{array}{c}\mathrm{t}=1 ; \mathrm{d}=2 \\
\text { Freq }\end{array}$ \\
\hline $\begin{array}{l}\mathrm{t}=1 ; \mathrm{d}=1 \\
\text { Bool }\end{array}$ & - & 0,6278 & 0,8562 & 0,9406 \\
\hline $\begin{array}{l}\mathrm{t}=1 ; \mathrm{d}=1 \\
\text { Freq }\end{array}$ & & - & 0,5128 & 0,3683 \\
\hline $\begin{array}{l}\mathrm{t}=1 ; \mathrm{d}=2 \\
\text { Bool }\end{array}$ & & & - & $-0,2568$ \\
\hline \hline
\end{tabular}

Tabela 6.37: Diferença em desvios-padrão com NCan_set e algoritmo SVM, com redução para 500 features

\subsubsection{Redução para 750 features}

Aplicou-se o script de redução para utilização do algoritmo SVM e redução para 750 features, utilizando-se NCan_set com kernel polinomial.

$\mathrm{Na}$ Tabela 6.38 são mostrados os resultados desse experimento, utilizando SVM, para representação booleana e freqüência. 


\begin{tabular}{|l||c|c|c|c|c|}
\hline \hline Algoritmo & Representação & $\begin{array}{c}\text { erro total } \\
(\%)\end{array}$ & $\begin{array}{c}\text { erro da } \\
\text { classe }+(\%)\end{array}$ & $\begin{array}{c}\text { cobertura } \\
(\%)\end{array}$ & $\begin{array}{c}F_{1} \\
(\%)\end{array}$ \\
\hline \hline SVM & Booleana & $9,11 \pm 7,75$ & $9,00 \pm 9,92$ & $91,96 \pm 11,33$ & $90,92 \pm 7,92$ \\
$(t=1 ; d=1)$ & Frequancla & $14,02 \pm 13,28$ & $11,87 \pm 14,00$ & $85,00 \pm 19,66$ & $85,13 \pm 14,99$ \\
\hline SVM & Booleana & $28,57 \pm 28,95$ & $16,68 \pm 11,63$ & $75,71 \pm 29,33$ & $77,44 \pm 22,65$ \\
$(\mathrm{t}=\mathrm{l} ; \mathrm{d}=2)$ & Freqüencla & $18,30 \pm 17,28$ & $17,36 \pm 17,01$ & $79,29 \pm 25,45$ & $79,73 \pm 21,09$ \\
\hline
\end{tabular}

Tabela 6.38: Experimento com NCan_set e algoritmo SVM, com redução para 750 features

A redução para 750 features corresponde a $36,3 \%$ do número total de atributos. Houve piora dos resultados, quando comparado com os resultados obtidos com redução para 500 features e representação booleana. O resultado para o erro total se manteve igual ao obtido com redução para 500 e 250 features com a representação por freqüência.

Nos resultados deste experimento pode ser observado que o erro total variou para todas as configurações do SVM, utilizando as duas representações com atribuição de peso booleano e de freqüência.

Neste experimento o menor erro total foi encontrado para a configuração default do SVM $(\mathrm{t}=1 ; \mathrm{d}=1)$ e representação booleana.

O erro da classe positiva obteve um comportamento semelhante ao erro total. Com relação à cobertura e $F_{1}$, neste experimento houve alterações significativas, principalmente para SVM com parâmetros $(\mathrm{t}=1 ; \mathrm{d}=2)$.

Baseado nas diferenças em desvios-padrão do erro total mostrados na Tabela 6.39, o maior valor encontrado é $\mathbf{0 , 9 1 8 3}$. Isso indica que o SVM com parâmetros $(t=1 ; d=1)$ e representação booleana possui melhor desempenho quando comparado com SVM com parâmetros $(t=1 ; d=2)$ e representação booleana.

\subsubsection{Redução para 1.000 features}

Aplicou-se o script de redução para utilização do algoritmo SVM e redução para 1.000 features, utilizando-se NCan_set com kernel polinomial.

Na Tabela 6.40 são mostrados os resultados desse experimento, utilizando SVM, para representação booleana e freqüência.

A redução para 1.000 features corresponde a 48,4\% do número total de atributos. 


\begin{tabular}{|l||c|c|c|c|}
\hline \hline Parâmetros & $\begin{array}{c}\mathrm{t}=1 ; \mathrm{d}=1 \\
\text { Bool }\end{array}$ & $\begin{array}{c}\mathrm{t}=1 ; \mathrm{d}=1 \\
\text { Freq }\end{array}$ & $\begin{array}{c}\mathrm{t}=1 ; \mathrm{d}=2 \\
\text { Bool }\end{array}$ & $\begin{array}{c}\mathrm{t}=1 ; \mathrm{d}=2 \\
\text { Freq }\end{array}$ \\
\hline $\begin{array}{l}\mathrm{t}=1 ; \mathrm{d}=1 \\
\text { Bool }\end{array}$ & - & 0,4516 & $\mathbf{0 , 9 1 8 3}$ & 0,6863 \\
\hline $\begin{array}{l}\mathrm{t}=1 ; \mathrm{d}=1 \\
\text { Freq }\end{array}$ & & - & 0,6460 & 0,2777 \\
\hline $\begin{array}{l}\mathrm{t}=1 ; \mathrm{d}=2 \\
\text { Bool }\end{array}$ & & & - & $-0,4308$ \\
\hline \hline
\end{tabular}

Tabela 6.39: Diferença em desvios-padrão com NCan_set e algoritmo SVM, com redução para 750 features

\begin{tabular}{|l||c|c|c|c|c|}
\hline \hline Algoritmo & Representaçāo & $\begin{array}{c}\text { erro total } \\
(\%)\end{array}$ & $\begin{array}{c}\text { erro da } \\
\text { classe }+(\%)\end{array}$ & $\begin{array}{c}\text { cobertura } \\
\text { (\%) }\end{array}$ & $\begin{array}{c}F_{\mathbf{I}} \\
(\%)\end{array}$ \\
\hline \hline SVM & Booleana & $9,20 \pm 7,89$ & $9,16 \pm 8,81$ & $91,79 \pm 11,88$ & $90,72 \pm 8,23$ \\
$(\mathrm{t}=\mathrm{l} ; \mathrm{d}=1)$ & Frequência & $14,02 \pm 13,28$ & $11,87 \pm 14,00$ & $85,00 \pm 19,66$ & $85,13 \pm 14,99$ \\
\hline SVM & Booleana & $28,57 \pm 28,95$ & $16,92 \pm 11,60$ & $74,46 \pm 29,63$ & $76,58 \pm 22,70$ \\
$(\mathrm{t}=1 ; \mathrm{d}=2)$ & Freqüencia & $19,55 \pm 16,23$ & $19,36 \pm 15,88$ & $79,29 \pm 25,45$ & $78,62 \pm 20,18$ \\
\hline
\end{tabular}

Tabela 6.40: Experimento com NCan_set e algoritmo SVM, com redução para 1.000 features

Houve pequena piora dos resultados, quando comparado com os resultados obtidos com redução para 750 features e representação booleana. O resultado para o erro total se manteve igual ao obtido com redução para 750, 500 e 250 features com a representação por freqüência. Ambas as análises foram feitas para SVM com parâmetros $(t=1 ; d=1)$.

Nos resultados deste experimento pode ser observado que o erro total variou para todas as configurações do SVM, utilizando as duas representações com atribuição de peso booleano e de freqüência.

Neste experimento o menor erro total foi encontrado para a configuração default do SVM $(t=1 ; d=1)$ e representação booleana.

O erro da classe positiva obteve um comportamento semelhante ao erro total. Com relação à cobertura e $F_{1}$, neste experimento houve alterações significativas, principalmente para SVM com parâmetros $(t=1 ; d=2)$.

Baseado nas diferenças em desvios-padrão do erro total mostrados na Tabela 6.41, o maior valor encontrado é 0,9129 . Isso indica que o SVM com parâmetros $(t=1 ; d=1)$ e representação booleana possui melhor desempenho quando comparado com SVM com 
parâmetros $(t=1 ; d=2)$ e representação booleana.

\begin{tabular}{|l||c|c|c|c|}
\hline \hline Paråmetros & $\begin{array}{c}t=1 ; d=1 \\
\text { Bool }\end{array}$ & $\begin{array}{c}t=1 ; d=1 \\
\text { Freq }\end{array}$ & $\begin{array}{c}t=1 ; d=2 \\
\text { Bool }\end{array}$ & $\begin{array}{c}t=1 ; d=2 \\
\text { Freq }\end{array}$ \\
\hline $\begin{array}{l}t=1 ; d=1 \\
\text { Bool }\end{array}$ & - & 0,4413 & 0,9129 & 0,8111 \\
\hline $\begin{array}{l}t=1 ; d=1 \\
\text { Freq }\end{array}$ & & - & 0,6460 & 0,3729 \\
\hline $\begin{array}{l}t=1 ; d=2 \\
\text { Bool }\end{array}$ & & & - & $-0,3843$ \\
\hline \hline
\end{tabular}

Tabela 6.41: Diferença em desvios-padrão com NCan_set e algoritmo SVM, com redução para 1.000 features

\subsubsection{Redução para 1.500 features}

Aplicou-se o script de redução para utilização do algoritmo SVM e redução para 1.500 features, utilizando-se NCan_set com kernel polinomial.

$\mathrm{Na}$ Tabela 6.42 são mostrados os resultados desse experimento, utilizando SVM, para representação booleana e freqüência.

\begin{tabular}{|l||c|c|c|c|c|}
\hline \hline Algorltmo & Representaçāo & $\begin{array}{c}\text { erro total } \\
(\%)\end{array}$ & $\begin{array}{c}\text { erro da } \\
\text { classe }+(\%)\end{array}$ & $\begin{array}{c}\text { cobertura } \\
(\%)\end{array}$ & $\begin{array}{c}\text { F }_{1} \\
(\%)\end{array}$ \\
\hline \hline SVM & Booleana & $11,88 \pm 11,93$ & $11,48 \pm 11,87$ & $87,86 \pm 16,43$ & $87,70 \pm 12,74$ \\
$(\mathrm{t}=1 ; \mathrm{d}=1)$ & Frequiêncla & $14,02 \pm 13,28$ & $11,87 \pm 14,00$ & $85,00 \pm 19,66$ & $85,13 \pm 14,99$ \\
\hline SVM & Booleana & $29,91 \pm 29,24$ & $18,58 \pm 14,94$ & $74,46 \pm 29,63$ & $76,22 \pm 23,37$ \\
$(\mathrm{t}=1 ; \mathrm{d}=2)$ & Frequỉencla & $19,64 \pm 16,23$ & $19,44 \pm 15,98$ & $79,29 \pm 25,45$ & $78,56 \pm 20,17$ \\
\hline \hline
\end{tabular}

Tabela 6.42: Experimento com NCan_set e algoritmo SVM, com redução para 1.500 features

A redução para 1.500 features corresponde a $72,5 \%$ do número total de atributos. Houve piora dos resultados, quando comparado com os resultados obtidos com redução para 1.000 features.

Nos resultados deste experimento pode ser observado que o erro total variou para todas as configurações do SVM, utilizando as duas representações com atribuição de peso booleano e de freqüência. 
Neste experimento o menor erro total foi encontrado para a configuração default do SVM $(t=1 ; d=1)$ e representação booleana.

O erro da classe positiva obteve um comportamento semelhante ao erro total. Com relação à cobertura e $F_{1}$, neste experimento houve alterações significativas, principalmente para SVM com parâmetros $(\mathrm{t}=1 ; \mathrm{d}=2)$.

Baseado nas diferenças em desvios-padrão do erro total mostrados na Tabela 6.43, o maior valor encontrado é $\mathbf{0 , 8 0 7 4}$. Isso indica que o SVM com parâmetros $(t=1 ; d=1)$ e representação booleana possui melhor desempenho quando comparado com SVM com parâmetros $(t=1 ; d=2)$ e representação booleana.

\begin{tabular}{|l||c|c|c|c|}
\hline \hline Parâmetros & $\begin{array}{c}t=1 ; d=1 \\
\text { Bool }\end{array}$ & $\begin{array}{c}t=1 ; d=1 \\
\text { Freq }\end{array}$ & $\begin{array}{c}t=1 ; d=2) \\
\text { Bool }\end{array}$ & $\begin{array}{c}t=1 ; d=2 \\
\text { Freq }\end{array}$ \\
\hline $\begin{array}{l}t=1 ; d=1 \\
\text { Bool }\end{array}$ & - & 0,1695 & 0,8074 & 0,5448 \\
\hline $\begin{array}{l}t=1 ; d=1 \\
\text { Freq }\end{array}$ & & - & 0,6997 & 0,3790 \\
\hline $\begin{array}{l}t=1 ; d=2 \\
\text { Bool }\end{array}$ & & & - & $-0,4343$ \\
\hline \hline
\end{tabular}

Tabela 6.43: Diferença em desvios-padrão com NCan_set e algoritmo SVM, com redução para 1.500 features 


\subsection{Resultados: Experimento 4 - NStem_set}

Os experimentos realizados com a identificação de atributos pelo método de stemming e utilizando-se das definições de pré-processamento baseado em (Weiss \& Indurkhya 1998) foram denominados de Experimento 4. O script ScpNStem gerou o conjunto de dados NStem_set.

Para o kernel polinomial ( $t=1)$, testou-se $\mathbf{d}$ com os valores 1 e 2. Não se utilizou parâmetros $t \neq 1$ com nenhuma redução de feature, o que implicaria em utilizar outros kernels que não o polinomial, pois não foi possível executar o algoritmo SVM com tais parâmetros.

\subsubsection{Sem Redução de features}

O número total de features desse experimento é de 1.956, conforme mostrado na Tabela 5.3 do Capítulo 5 .

Na Tabela 6.44 são mostrados os resultados para NStem_set, sem redução de features, com o algoritmo SVM e kernel polinomial.

\begin{tabular}{|l||c|c|c|c|c|}
\hline \hline Algoritmo & Representaçäo & $\begin{array}{c}\text { erro total } \\
(\%)\end{array}$ & $\begin{array}{c}\text { erro da } \\
\text { classe }+(\%)\end{array}$ & $\begin{array}{c}\text { cobertura } \\
(\%)\end{array}$ & $\begin{array}{c}F_{1} \\
(\%)\end{array}$ \\
\hline \hline SVM & Booleana & $4,68 \pm 3,24$ & $4,72 \pm 6,11$ & $94,64 \pm 6,94$ & $94,70 \pm 4,11$ \\
$(t=1 ; d=1)$ & Freqüêncla & $3,30 \pm 4,57$ & $1,25 \pm 3,95$ & $94,82 \pm 8,93$ & $96,40 \pm 5,03$ \\
\hline SVM & Booleana & $7,98 \pm 6,25$ & $8,80 \pm 9,95$ & $94,64 \pm 6,94$ & $92,50 \pm 6,12$ \\
$(\mathbf{t}=\mathbf{1} ; \mathbf{d}=\mathbf{2})$ & Freqüêncla & $7,95 \pm 6,78$ & $4,72 \pm 8,05$ & $89,64 \pm 13,41$ & $91,53 \pm 7,73$ \\
\hline \hline
\end{tabular}

Tabela 6.44: Experimento com NStem_set e algoritmo SVM, sem redução de features

Nos resultados deste experimento pode ser observado que o erro total variou para todas as configurações do SVM quando utilizado a representação com atribuição de peso booleano e freqüência.

Neste experimento o menor erro total foi encontrado para a configuração default do SVM $(t=1 ; d=1)$ e representação por freqüência.

Com relação ao erro da classe positiva não se obteve um comportamento semelhante ao erro total, apesar dos dados terem vindo de uma classe bivalorada, balanceada. Ocorreu erro da classe positiva superiores aos do erro total. Com relação a cobertura e $F_{1}$ não se 
pode tirar grandes conclusões, uma vez que a variação entre os dados foi muito pequena.

Baseado nas diferenças em desvios-padrão do erro total mostrados na Tabela 6.45, o maior valor encontrado é $\mathbf{0 , 8 1 1 9}$. Isso indica que o SVM com parâmetros $(t=1 ; d=1)$ e representação de freqüência possui melhor desempenho quando comparado com SVM com parâmetros $(\mathrm{t}=1 ; \mathrm{d}=2)$ e representação booleana.

\begin{tabular}{|l||c|c|c|c|}
\hline \hline Parâmetros & $\begin{array}{c}t=1 ; d=1 \\
\text { Bool }\end{array}$ & $\begin{array}{c}t=1 ; d=1 \\
\text { Freq }\end{array}$ & $\begin{array}{c}t=1 ; d=2 \\
\text { Bool }\end{array}$ & $\begin{array}{c}t=1 ; d=2 \\
\text { Freq }\end{array}$ \\
\hline $\begin{array}{l}t=1 ; d=1 \\
\text { Bool }\end{array}$ & - & $-0,3484$ & 0,6233 & 0,6154 \\
\hline $\begin{array}{l}t=1 ; d=1 \\
\text { Freq }\end{array}$ & & - & 0,8119 & 0,8043 \\
\hline $\begin{array}{l}t=1 ; d=2 \\
\text { Bool }\end{array}$ & & & - & $-0,0044$ \\
\hline \hline
\end{tabular}

Tabela 6.45: Diferença em desvios-padrão com NStem_set e algoritmo SVM, sem redução de features

\subsubsection{Redução para 250 features}

$\mathrm{Na}$ Tabela 6.46 são mostrados os resultados para NStem_set e redução para 250 features, com o algoritmo SVM e kernel polinomial.

\begin{tabular}{|c|c|c|c|c|c|}
\hline Algorltmo & Representação & $\begin{array}{c}\text { erro total } \\
(\%)\end{array}$ & $\begin{array}{c}\text { erro da } \\
\text { classe }+(\%)\end{array}$ & $\begin{array}{c}\text { cobertura } \\
(\%)\end{array}$ & $\begin{array}{l}F_{1} \\
(\%) \\
\end{array}$ \\
\hline $\begin{array}{l}\text { SVM } \\
(t=1 ; d=1)\end{array}$ & $\begin{array}{c}\text { Booleana } \\
\text { Frequiêncla }\end{array}$ & $\begin{array}{l}6,43 \pm 6,60 \\
5,36 \pm 6,07\end{array}$ & $\begin{array}{l}6,86 \pm 7,61 \\
3,93 \pm 6,35\end{array}$ & $\begin{array}{c}95,00 \pm 12,08 \\
93,39 \pm 9,15\end{array}$ & $\begin{array}{l}93,41 \pm 7,46 \\
94,46 \pm 6,33\end{array}$ \\
\hline $\begin{array}{l}\text { SVM } \\
(t=1 ; d=2)\end{array}$ & $\begin{array}{l}\text { Booleana } \\
\text { Frequência }\end{array}$ & $\begin{array}{l}7,86 \pm 5,12 \\
8,57 \pm 6,23\end{array}$ & $\begin{array}{l}5,47 \pm 9,13 \\
3,93 \pm 6,35\end{array}$ & $\begin{array}{c}90,89 \pm 8,64 \\
86,96 \pm 12,26\end{array}$ & $\begin{array}{l}92,09 \pm 4,96 \\
90,68 \pm 7,30\end{array}$ \\
\hline
\end{tabular}

Tabela 6.46: Experimento com NStem_set e algoritmo SVM, com redução para 250 features

A redução para 250 features corresponde a $12,8 \%$ do número total de atributos. Houve piora dos resultados, quando comparado com os resultados obtidos sem nenhuma redução de features.

Nos resultados deste experimento pode ser observado que o erro total variou para todas as configurações do SVM quando utilizado a representação com atribuição de peso 
booleano e freqüência.

Neste experimento o menor erro total foi encontrado para a configuração default do SVM $(t=1 ; d=1)$ e representação por freqüência, semelhante ao que ocorreu com o experimento sem redução de parâmetros.

Com relação ao erro da classe positiva se obteve um comportamento semelhante ao erro total, com os dados são oriundos de uma classe bivalorada, balanceada. Com relação a cobertura e $F_{1}$ não se pode tirar grandes conclusões, uma vez que a variação entre os dados foi muito pequena.

Baseado nas diferenças em desvios-padrão do erro total mostrados na Tabela 6.47 , o maior valor encontrado é $\mathbf{0 , 5 2 1 9}$. Isso indica que o SVM com parâmetros $(t=1 ; d=1)$ e representação de freqüência possui melhor desempenho quando comparado com SVM com parâmetros $(t=1 ; d=2)$ e representação de freqüência.

\begin{tabular}{|l||c|c|c|c|}
\hline \hline Parámetros & $\begin{array}{c}t=1 ; d=1 \\
\text { Bool }\end{array}$ & $\begin{array}{c}t=1 ; d=1 \\
\text { Freq }\end{array}$ & $\begin{array}{c}t=1 ; d=2 \\
\text { Bool }\end{array}$ & $\begin{array}{c}t=1 ; d=2 \\
\text { Freq }\end{array}$ \\
\hline $\begin{array}{l}t=1 ; d=1 \\
\text { Bool }\end{array}$ & - & $-0,1688$ & 0,2421 & 0,3335 \\
\hline $\begin{array}{l}t=1 ; d=1 \\
\text { Freq }\end{array}$ & & - & 0,4452 & $\mathbf{0 , 5 2 1 9}$ \\
\hline $\begin{array}{l}t=1 ; d=2 \\
\text { Bool }\end{array}$ & & & - & 0,1245 \\
\hline \hline
\end{tabular}

Tabela 6.47: Diferença em desvios-padrão com NStem_set e algoritmo SVM, com redução para 250 features

\subsubsection{Redução para 500 features}

Aplicou-se o script de redução para utilização do algoritmo SVM e redução para 500 features, utilizando-se NStem_set com kernel polinomial.

$\mathrm{Na}$ Tabela 6.48 são mostrados os resultados para NStem_set, com redução para 500 features.

A redução para 500 features corresponde a $25,6 \%$ do número total de atributos. Houve melhora dos resultados, quando comparado com os resultados obtidos com redução para 250 features e sem redução de features. 


\begin{tabular}{|l||c|c|c|c|c|}
\hline \hline Algorltmo & Representacāo & $\begin{array}{c}\text { erro total } \\
(\%)\end{array}$ & $\begin{array}{c}\text { erro da } \\
\text { classe }+(\%)\end{array}$ & $\begin{array}{c}\text { cobertura } \\
(\%)\end{array}$ & $\begin{array}{c}F_{1} \\
(\%)\end{array}$ \\
\hline \hline SVM & Booleana & $5,93 \pm 4,68$ & $5,97 \pm 6,32$ & $94,82 \pm 8,93$ & $94,04 \pm 4,97$ \\
$(t=1 ; d=1)$ & Freqüëncla & $2,68 \pm 3,47$ & $1,25 \pm 3,95$ & $96,07 \pm 6,35$ & $97,23 \pm 3,59$ \\
\hline SVM & Booleana & $6,02 \pm 5,87$ & $4,22 \pm 8,92$ & $93,57 \pm 9,00$ & $94,11 \pm 5,62$ \\
$(t=1 ; d=2)$ & Freqüüncla & $9,20 \pm 6,29$ & $4,90 \pm 8,26$ & $87,14 \pm 12,25$ & $90,23 \pm 6,83$ \\
\hline \hline
\end{tabular}

Tabela 6.48: Experimento com NStem_set e algoritmo SVM, com redução para 500 features

Nos resultados deste experimento pode ser observado que o erro total variou para todas as configurações do SVM quando utilizado a representação com atribuição de peso booleano e freqüência.

Neste experimento o menor erro total foi encontrado para a configuraçāo default do SVM $(t=1 ; d=1)$ e representação por freqüência, semelhante ao que ocorreu com o experimento sem redução de parâmetros. O erro deste experimento para SVM $(t=1 ; d=1)$ e representação por freqüência é o menor erro total para o conjunto de dados NStem_set.

Com relaçāo ao erro da classe positiva se obteve um comportamento semelhante ao erro total, com os dados são oriundos de uma classe bivalorada, balanceada. Com relação a cobertura e $F_{1}$ não se pode tirar grandes conclusōes, uma vez que a variaçāo entre os dados foi muito pequena.

Baseado nas diferenças em desvios-padrão do erro total mostrados na Tabela 6.49, o maior valor encontrado é 1,2836 . Isso indica que o $S V M$ com parâmetros $(t=1 ; d=1)$ e representação de freqüência possui melhor desempenho quando comparado com SVM com parâmetros $(t=1 ; d=2)$ e representação de freqüência.

\begin{tabular}{|l||c|c|c|c|}
\hline \hline Parâmetros & $\begin{array}{c}t=1 ; d=1 \\
\text { Bool }\end{array}$ & $\begin{array}{c}t=1 ; d=1 \\
\text { Freq }\end{array}$ & $\begin{array}{c}t=1 ; d=2 \\
\text { Bool }\end{array}$ & $\begin{array}{c}t=1 ; d=2 \\
\text { Freq }\end{array}$ \\
\hline \hline $\begin{array}{l}t=1 ; d=1 \\
\text { Bool }\end{array}$ & - & $-0,7889$ & 0,0170 & 0,5898 \\
\hline $\begin{array}{l}t=1 ; d=1 \\
\text { Freq }\end{array}$ & & - & 0,6927 & 1,2836 \\
\hline $\begin{array}{l}t=1 ; d=2 \\
\text { Bool }\end{array}$ & & & - & 0,5227 \\
\hline \hline
\end{tabular}

Tabela 6.49: Diferença em desvios-padrāo com NStem_set e algoritmo SVM, com redução para 500 features 
$\mathrm{Na}$ Tabela 6.50 são mostrados todos os resultados comparativos para os algoritmos da ferramenta Weka, e o algoritmo SVM $(t=1 ; \mathrm{d}=1)$ utilizando-se de NStem_set, com redução para 500 features.

\begin{tabular}{|c|c|c|c|c|c|}
\hline Algoritmo & Representação & $\begin{array}{c}\text { erro total } \\
(\%)\end{array}$ & $\begin{array}{c}\text { erro da } \\
\text { classe }+(\%)\end{array}$ & $\begin{array}{c}\text { cobertura } \\
(\%)\end{array}$ & $\begin{array}{r}F_{1} \\
(\%)\end{array}$ \\
\hline $\begin{array}{l}\text { SVM } \\
(t=1 ; d=1)\end{array}$ & $\begin{array}{c}\text { Booleana } \\
\text { Freqüiêncla }\end{array}$ & $\begin{array}{l}5,93 \pm 4,68 \\
2,68 \pm 3,47 \\
\end{array}$ & $\begin{array}{l}5,97 \pm 6,32 \\
1,25 \pm 3,95 \\
\end{array}$ & $\begin{array}{l}94,82 \pm 8,93 \\
96,07 \pm 6,35 \\
\end{array}$ & $\begin{array}{l}94,04 \pm 4,97 \\
97,23 \pm 3,59 \\
\end{array}$ \\
\hline $\begin{array}{l}\text { Nalve } \\
\text { Bayes }\end{array}$ & $\begin{array}{c}\text { Booleana } \\
\text { Freqüência }\end{array}$ & $\begin{array}{c}10,57 \pm 6,93 \\
18,93 \pm 11,44\end{array}$ & $\begin{array}{c}6,15 \pm 8,38 \\
16,63 \pm 10,65\end{array}$ & $\begin{array}{l}85,71 \pm 11,39 \\
77,67 \pm 24,08 \\
\end{array}$ & $\begin{array}{c}89,02 \pm 7,09 \\
78,55 \pm 17,23 \\
\end{array}$ \\
\hline $\begin{array}{l}\text { Vizinho } \\
\text { mais Próximo }\end{array}$ & $\begin{array}{c}\text { Booleana } \\
\text { Freqüencla }\end{array}$ & $\begin{array}{l}17,43 \pm 10,42 \\
39,20 \pm 14,60\end{array}$ & $\begin{array}{l}18,73 \pm 12,91 \\
42,70 \pm 10,68\end{array}$ & $\begin{array}{l}87,14 \pm 14,82 \\
89,46 \pm 14,23\end{array}$ & $\begin{array}{c}83,00 \pm 9,16 \\
69,65 \pm 11,47\end{array}$ \\
\hline $\begin{array}{l}\text { Árvore } \\
\text { de Decisão }\end{array}$ & $\begin{array}{c}\text { Booleana } \\
\text { Freqüuêncla }\end{array}$ & $\begin{array}{r}11,86 \pm 8,36 \\
11,52 \pm 12,64 \\
\end{array}$ & $\begin{array}{r}9,16 \pm 11,85 \\
11,47 \pm 11,73 \\
\end{array}$ & $\begin{array}{l}88,57 \pm 12,46 \\
88,75 \pm 18,11 \\
\end{array}$ & $\begin{array}{r}88,83 \pm 8,70 \\
87,97 \pm 14,15 \\
\end{array}$ \\
\hline $\begin{array}{l}\text { Regras } \\
\text { de Declsão }\end{array}$ & $\begin{array}{c}\text { Booleana } \\
\text { Freqüêncla }\end{array}$ & $\begin{array}{l}23,63 \pm 10,22 \\
22,32 \pm 12,77\end{array}$ & $\begin{array}{l}22,37 \pm 15,96 \\
25,52 \pm 13,36 \\
\end{array}$ & $\begin{array}{l}84,46 \pm 15,64 \\
89,64 \pm 12,05\end{array}$ & $\begin{array}{l}78,72 \pm 7,79 \\
80,52 \pm 10,21\end{array}$ \\
\hline $\begin{array}{l}\text { Tabela } \\
\text { de Declsão }\end{array}$ & $\begin{array}{c}\text { Booleana } \\
\text { Frequiencla }\end{array}$ & $\begin{array}{l}7,98 \pm 9,30 \\
7,14 \pm 7,47\end{array}$ & $\begin{array}{l}7,91 \pm 11,81 \\
8,19 \pm 10,70\end{array}$ & $\begin{array}{l}93,57 \pm 9,00 \\
96,25 \pm 8,44\end{array}$ & $\begin{array}{l}92,45 \pm 8,68 \\
93,32 \pm 6,40 \\
\end{array}$ \\
\hline
\end{tabular}

Tabela 6.50: Experimento com NStem_set usando a ferramenta Weka e os diferentes algoritmos e algoritmo SVM, com redução para 500 features

Calculando a diferença em desvios-padrão temos os resultados mostrados nas Tabelas 6.51 e 6.52, para NStem_set com o algoritmo SVM $(\mathrm{t}=1 ; \mathrm{d}=1)$, que gerou melhor classificador entre as diferentes configuraçōes do SVM e os algoritmos da ferramenta Weka, com a representação booleana e de freqüência.

Baseado nas diferenças em desvios-padrão o maior valor encontrado é 2,7451, como mostrado na Tabela 6.51. O SVM com parâmetros $(t=1 ; d=1)$ e representação de freqüência possue melhor desempenho que Regras de Decisão com representação booleana, com nível de confiança de $95 \%$.

Baseado nas diferenças em desvios-padrão o maior valor encontrado é $\mathbf{- 2 , 7 6 4 6 \text { , }}$ como mostrado na Tabela 6.9. A Tabela de Decisão com representação de freqüência possue melhor desempenho que Vizinho mais Próximo com representação de freqüência, com nível de confiança de $95 \%$.

O gráfico ilustrado na Figura 6.7 mostra os resultados obtidos com SVM e os algoritmos da ferramenta Weka, utilizando NStem_Bool_set e NStem_Freq_set, com redução para 500 features, referentes a Tabela 6.50 . 


\begin{tabular}{|l||c|c|c|c|c|c|}
\hline \hline Algorltmo & $\begin{array}{c}\text { SVM } \\
(t=1 ; d=1) \\
\text { Bool }\end{array}$ & $\begin{array}{c}\text { SVM } \\
(t=1 ; d=1) \\
\text { Freq }\end{array}$ & $\begin{array}{c}\text { Regra de } \\
\text { Decisāo } \\
\text { Bool }\end{array}$ & $\begin{array}{c}\text { Regra de } \\
\text { Decisão } \\
\text { Freq }\end{array}$ & $\begin{array}{c}\text { Tabela de } \\
\text { Decisão } \\
\text { Bool }\end{array}$ & $\begin{array}{c}\text { Tabela de } \\
\text { Declsāo } \\
\text { Freq }\end{array}$ \\
\hline $\begin{array}{l}\text { SVM(t=1;d=1) } \\
\text { Bool }\end{array}$ & - & $-0,7889$ & 2,2269 & 1,7043 & 0,2785 & 0,1941 \\
\hline $\begin{array}{l}\text { SVM(t=1;d=1) } \\
\text { Freq }\end{array}$ & & - & $\mathbf{2 , 7 4 5 1}$ & 2,0989 & 0,7551 & 0,7658 \\
\hline $\begin{array}{l}\text { Regra de Decisāo } \\
\text { Bool }\end{array}$ & & & - & $-0,1133$ & $-1,6017$ & $-1,8422$ \\
\hline $\begin{array}{l}\text { Regra de Decisão } \\
\text { Freq }\end{array}$ & & & & - & $-1,2837$ & $-1,4511$ \\
\hline $\begin{array}{l}\text { Tabela de Decisăo } \\
\text { Bool }\end{array}$ & & & & & - & $-0,0996$ \\
\hline \hline
\end{tabular}

Tabela 6.51: Diferença em desvios-padrão com NStem_set, algoritmo SVM e os algoritmos da Weka, com redução para 500 features

\subsubsection{Redução para 750 features}

Aplicou-se o script de redução para utilização do algoritmo SVM e redução para 750 features, utilizando-se NStem_set com kernel polinomial.

$\mathrm{Na}$ Tabela 6.53 são mostrados os resultados desse experimento, utilizando SVM, para representação booleana e freqüência.

A redução para 750 features corresponde a $38,3 \%$ do número total de atributos. Houve piora dos resultados com representação de freqüência, quando comparado com os resultados obtidos com redução para 500 features.

Nos resultados deste experimento pode ser observado que o erro total variou para todas as configurações do SVM quando utilizado a representação com atribuição de peso booleano e freqüência.

Neste experimento o menor erro total foi encontrado para a configuração default do SVM $(\mathrm{t}=1 ; \mathrm{d}=1)$ e representação por freqüência, semelhante ao que ocorreu com o experimento sem redução de parâmetros.

Com relação ao erro da classe positiva se obteve um comportamento semelhante ao erro total. Com relação à cobertura e $F_{1}$, não se pode tirar grandes conclusões, uma vez que a variação entre os dados foi muito pequena.

Baseado nas diferenças em desvios-padrão do erro total mostrados na Tabela 6.54, 


\begin{tabular}{|c|c|c|c|c|c|c|c|c|}
\hline Algoritmo & $\begin{array}{c}\text { Naive } \\
\text { Bayes } \\
\text { Bool }\end{array}$ & $\begin{array}{l}\text { Nalve } \\
\text { Bayes } \\
\text { Freq }\end{array}$ & $\begin{array}{c}\text { Vizinho } \\
+ \text { Próximo } \\
\text { Bool }\end{array}$ & $\begin{array}{c}\text { Vizinho } \\
+ \text { Próximo } \\
\text { Freq }\end{array}$ & $\begin{array}{c}\text { Árvore } \\
\text { Decisāo } \\
\text { Bool }\end{array}$ & $\begin{array}{c}\text { Árvore } \\
\text { Decisão } \\
\text { Freq }\end{array}$ & $\begin{array}{c}\text { Tabela de } \\
\text { Decisảo } \\
\text { Bool }\end{array}$ & $\begin{array}{c}\text { Tabela de } \\
\text { Decisäo } \\
\text { Freq }\end{array}$ \\
\hline $\begin{array}{l}\text { Nalve } \\
\text { Bool }\end{array}$ & - & 0,8839 & 0,7752 & 2,5053 & 0,1680 & 0,0932 & $-0,3158$ & $-0,4761$ \\
\hline $\begin{array}{l}\text { Nalve } \\
\text { Freq }\end{array}$ & & . & $-0,1371$ & 1,5455 & $-0,7057$ & $-0,6147$ & $-1,0504$ & $-1,2204$ \\
\hline $\begin{array}{l}\text { Vizinho } \\
\text { Bool }\end{array}$ & & & - & 1,7164 & $-0,5896$ & $-0,5102$ & $-0,9569$ & $-1,1350$ \\
\hline $\begin{array}{l}\text { Vizinho } \\
\text { Freq }\end{array}$ & & & & - & $-2,2982$ & $-2,0271$ & $-2,5506$ & $-2,7646$ \\
\hline $\begin{array}{l}\text { Árvore } \\
\text { Bool }\end{array}$ & & & & & - & $-0,0317$ & $-0,4388$ & $-0,5954$ \\
\hline $\begin{array}{l}\text { Árvore } \\
\text { Freq }\end{array}$ & & & & & & - & $-0,3190$ & $-0,4219$ \\
\hline $\begin{array}{l}\text { Tabela } \\
\text { Bool }\end{array}$ & & & & & & & - & $-0,0996$ \\
\hline
\end{tabular}

Tabela 6.52: Diferença em desvios-padrão com NStem_set, algoritmo SVM e os algoritmos da Weka, com redução para 500 features

\begin{tabular}{|l||c|c|c|c|c|}
\hline \hline Algoritmo & Representação & $\begin{array}{c}\text { erro total } \\
(\%)\end{array}$ & $\begin{array}{c}\text { erro da } \\
\text { classe }+(\%)\end{array}$ & $\begin{array}{c}\text { cobertura } \\
(\%)\end{array}$ & $\begin{array}{c}F_{1} \\
(\%)\end{array}$ \\
\hline \hline SVM & Booleana & $5,89 \pm 4,67$ & $3,61 \pm 5,83$ & $92,32 \pm 8,87$ & $93,96 \pm 4,98$ \\
$(t=1 ; d=1)$ & Frequeêncla & $4,02 \pm 5,67$ & $1,25 \pm 3,95$ & $93,39 \pm 11,36$ & $95,57 \pm 6,45$ \\
\hline SVM & Booleana & $7,98 \pm 6,66$ & $6,44 \pm 10,39$ & $92,14 \pm 9,00$ & $92,24 \pm 6,29$ \\
$(t=1 ; d=2)$ & Frequeencla & $7,32 \pm 5,91$ & $4,72 \pm 8,05$ & $90,89 \pm 10,96$ & $92,40 \pm 6,24$ \\
\hline \hline
\end{tabular}

Tabela 6.53: Experimento com NStem_set e algoritmo SVM, com redução para 750 features

o melhor valor encontrado é $\mathbf{0 , 6 4 0 3}$. Isso indica que o SVM com parâmetros $(t=1 ; d=1)$ e representação de freqüência possui desempenho ligeiramente melhor quando comparado com SVM com parâmetros $(t=1 ; d=2)$ e representação booleana. 


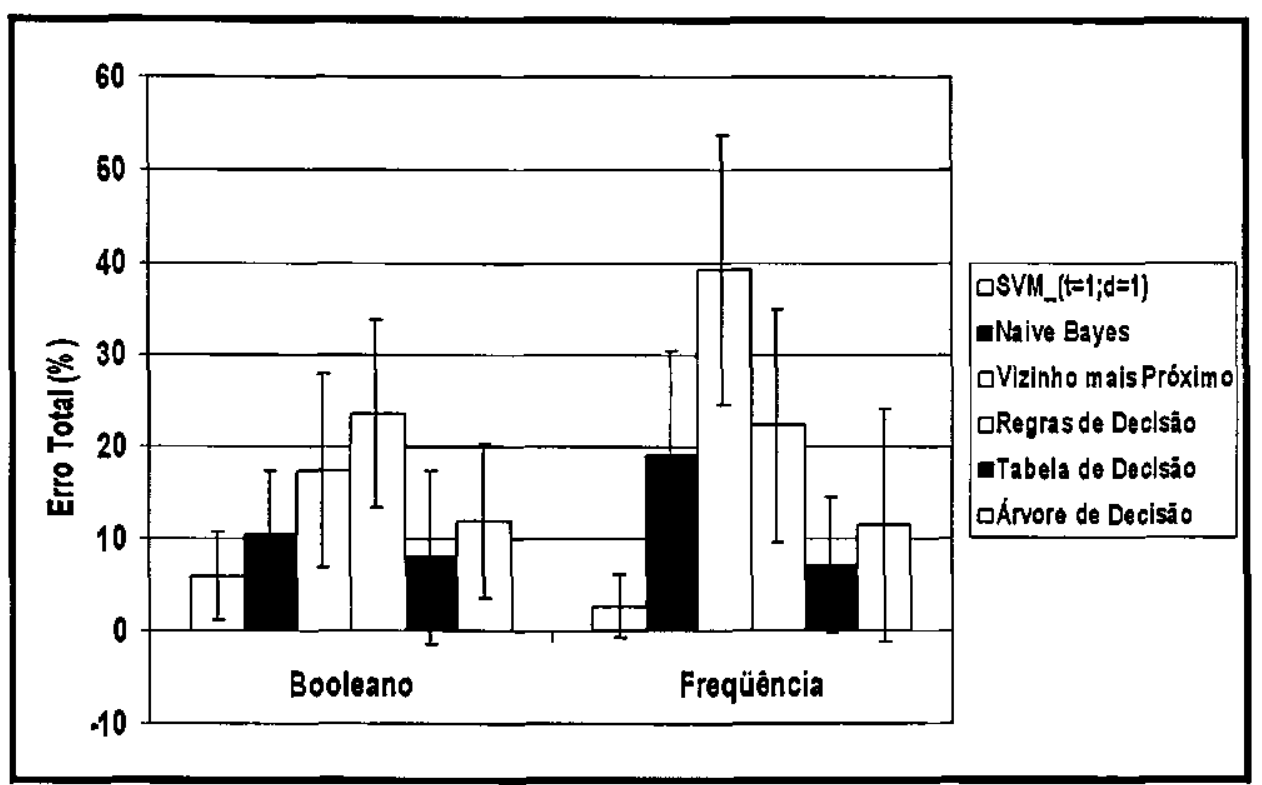

Figura 6.7: Comparações dos resultados obtidos (Erro Total) com SVM e os algoritmos da ferramenta Weka para o conjunto de dados NStem_set com redução para 500 features

\begin{tabular}{|l||c|c|c|c|}
\hline Parâmetros & $\begin{array}{c}\mathrm{t}=1 ; \mathrm{d}=1 \\
\text { Bool }\end{array}$ & $\begin{array}{c}\mathrm{t}=1 ; \mathrm{d}=1 \\
\text { Freq }\end{array}$ & $\begin{array}{c}\mathrm{t}=1 ; \mathrm{d}=2 \\
\text { Bool }\end{array}$ & $\begin{array}{c}\mathrm{t}=1 ; \mathrm{d}=2 \\
\text { Freq }\end{array}$ \\
\hline \hline $\begin{array}{l}\mathrm{t}=1 ; \mathrm{d}=1 \\
\text { Bool }\end{array}$ & - & $-0,3600$ & 0,3634 & 0,2685 \\
\hline $\begin{array}{l}\mathrm{t}=1 ; \mathrm{d}=1 \\
\text { Freq }\end{array}$ & & - & $\mathbf{0 , 6 4 0 3}$ & 0,5698 \\
\hline $\begin{array}{l}\mathrm{t}=1 ; \mathrm{d}=2 \\
\text { Bool }\end{array}$ & & & - & $-0,1048$ \\
\hline \hline
\end{tabular}

Tabela 6.54: Diferença em desvios-padrão com NStem_set e algoritmo SVM, com redução para 750 features 


\subsubsection{Redução para 1.000 features}

Aplicou-se o script de redução para utilização do algoritmo SVM e redução para 1.000 features, utilizando-se NStem_set com kernel polinomial.

Na Tabela 6.55 são mostrados os resultados desse experimento, utilizando SVM, para representação booleana e freqüência.

\begin{tabular}{|l||c|c|c|c|c|}
\hline \hline Algorltmo & Representaçāo & $\begin{array}{c}\text { erro total } \\
(\%)\end{array}$ & $\begin{array}{c}\text { erro da } \\
\text { classe }+(\%)\end{array}$ & $\begin{array}{c}\text { cobertura } \\
(\%)\end{array}$ & $\begin{array}{c}\mathbf{F}_{\mathbf{1}} \\
(\%)\end{array}$ \\
\hline \hline SVM & Booleana & $5,89 \pm 4,67$ & $4,72 \pm 6,11$ & $93,57 \pm 9,00$ & $94,04 \pm 4,97$ \\
$(\mathbf{t = 1 ; d = 1 )}$ & Freqüuencla & $3,39 \pm 4,92$ & $1,25 \pm 3,95$ & $94,64 \pm 9,67$ & $96,33 \pm 5,54$ \\
\hline SVM & Booleana & $7,98 \pm 6,66$ & $6,44 \pm 10,39$ & $92,14 \pm 9,00$ & $92,24 \pm 6,29$ \\
$(\mathbf{t = 1 ; d = 2 )}$ & Frequêncla & $6,61 \pm 6,35$ & $3,47 \pm 7,67$ & $\mathbf{9 0 , 8 9 \pm 1 0 , 9 6}$ & $\mathbf{9 3 , 0 7 \pm 6 , 6 9}$ \\
\hline \hline
\end{tabular}

Tabela 6.55: Experimento com NStem_set e algoritmo SVM, com redução para 1.000 features

A redução para 1.000 features corresponde a $51,1 \%$ do número total de atributos. Houve pequena melhora dos resultados, quando comparado com os resultados obtidos com redução para 750 features.

Nos resultados deste experimento pode ser observado que o erro total variou para todas as configurações do SVM quando utilizado a representação com atribuição de peso booleano e freqüência.

Neste experimento o menor erro total foi encontrado para a configuração default do SVM $(\mathrm{t}=1 ; \mathrm{d}=1)$ e representação por freqüência. O erro deste experimento para SVM $(t=1 ; d=1)$ e representação por freqüência é também, semelhante a reduçāo para 500 features, o menor erro total para o conjunto de dados NStem_set.

Com relação ao erro da classe positiva se obteve um comportamento semelhante ao erro total. Com relação à cobertura e $F_{1}$, não se pode tirar grandes conclusões, uma vez que a variação entre os dados foi muito pequena.

Baseado nas diferenças em desvios-padrão do erro total mostrados na Tabela 6.56, o maior valor encontrado é $\mathbf{0 , 7 8 3 9}$. Isso indica que o SVM $(t=1 ; d=1)$ e representação de freqüência possui melhor desempenho quando comparado com SVM com parâmetros $(t=1 ; d=2)$ e representação booleana. 


\begin{tabular}{|l||c|c|c|c|}
\hline \hline Parâmetros & $\begin{array}{c}t=1 ; d=1 \\
\text { Bool }\end{array}$ & $\begin{array}{c}t=1 ; d=1 \\
\text { Freq }\end{array}$ & $\begin{array}{c}t=1 ; d=2 \\
\text { Bool }\end{array}$ & $\begin{array}{c}t=1 ; d=2 \\
\text { Freq }\end{array}$ \\
\hline \hline $\begin{array}{l}t=1 ; d=1 \\
\text { Bool }\end{array}$ & - & $-0,5212$ & 0,3634 & 0,1292 \\
\hline $\begin{array}{l}t=1 ; d=1 \\
\text { Freq }\end{array}$ & & - & 0,7839 & 0,5669 \\
\hline $\begin{array}{l}t=1 ; d=2 \\
\text { Bool }\end{array}$ & & & - & $-0,2105$ \\
\hline \hline
\end{tabular}

Tabela 6.56: Diferença em desvios-padrão com NStem_set e algoritmo SVM, com redução para 1.000 features

\subsubsection{Redução para 1.500 features}

Aplicou-se o script de redução para utilização do algoritmo SVM e redução para 1.500 features, utilizando-se NStem_set com kernel polinomial.

Na Tabela 6.57 são mostrados os resultados desse experimento, utilizando SVM com parâmetros, para representação booleana e freqüência.

\begin{tabular}{|l||c|c|c|c|c|}
\hline \hline Algoritmo & Representação & $\begin{array}{c}\text { erro total } \\
(\%)\end{array}$ & $\begin{array}{c}\text { erro da } \\
\text { classe }+(\%)\end{array}$ & $\begin{array}{c}\text { cobertura } \\
(\%)\end{array}$ & $\begin{array}{c}\text { F }_{\mathbf{1}} \\
(\%)\end{array}$ \\
\hline \hline SVM & Booleana & $5,89 \pm 4,67$ & $4,72 \pm 6,11$ & $93,57 \pm 9,00$ & $94,04 \pm 4,97$ \\
$(t=1 ; d=1)$ & Freqüência & $2,68 \pm 3,47$ & $1,25 \pm 3,95$ & $96,07 \pm 6,35$ & $\mathbf{9 7 , 2 3 \pm 3 , 5 9}$ \\
\hline SVM & Booleana & $8,56 \pm 6,87$ & $8,80 \pm 9,95$ & $93,57 \pm 9,00$ & $91,84 \pm 6,49$ \\
$(t=1 ; d=2)$ & Frequêncla & $7,32 \pm 5,91$ & $4,72 \pm 8,05$ & $90,89 \pm 10,96$ & $92,40 \pm 6,24$ \\
\hline
\end{tabular}

Tabela 6.57: Experimento com NStem_set e algoritmo SVM, com redução para 1.500 features

A redução para 1.500 features corresponde a $76,7 \%$ do número total de atributos. Houve melhora dos resultados, quando comparado com os resultados obtidos com redução para 1.000 features.

Nos resultados deste experimento pode ser observado que o erro total variou para todas as configurações do SVM quando utilizado a representação com atribuição de peso booleano e freqüência.

Neste experimento o menor erro total foi encontrado para a configuração default do SVM $(t=1 ; d=1)$ e representação por freqüência.

Com relação ao erro da classe positiva se obteve um comportamento semelhante ao 
erro total, com os dados são oriundos de uma classe bivalorada, balanceada. Com relação a cobertura e $F_{1}$ não se pode tirar grandes conclusões, uma vez que a variação entre os dados foi muito pequena.

Baseado nas diferenças em desvios-padrão do erro total mostrados na Tabela 6.58, o maior valor encontrado é 1,0804. Isso indica que o SVM com parâmetros $(t=1 ; d=1)$ e representação de freqüência possui melhor desempenho quando comparado com SVM com parâmetros $(t=1 ; d=2)$ e representação booleana .

\begin{tabular}{|l||c|c|c|c|}
\hline \hline Parămetros & $\begin{array}{c}t=1 ; d=1 \\
\text { Bool }\end{array}$ & $\begin{array}{c}t=1 ; d=1 \\
\text { Freq }\end{array}$ & $\begin{array}{c}t=1 ; d=2 \\
\text { Bool }\end{array}$ & $\begin{array}{c}t=1 ; d=2 \\
\text { Freq }\end{array}$ \\
\hline $\begin{array}{l}t=1 ; d=1 \\
\text { Bool }\end{array}$ & - & $-0,7803$ & 0,4546 & 0,2685 \\
\hline $\begin{array}{l}t=1 ; d=1 \\
\text { Freq }\end{array}$ & & - & 1,0804 & 0,9575 \\
\hline $\begin{array}{l}t=1 ; d=2 \\
\text { Bool }\end{array}$ & & & - & $-0,1935$ \\
\hline \hline
\end{tabular}

Tabela 6.58: Diferença em desvios-padrão com NStem_set e algoritmo SVM, com redução para 1.500 features 


\subsection{Síntese dos Experimentos}

Na Tabela 6.59 são mostrados os resultados dos 4 experimentos, utilizando SVM com parâmetros default $(\mathrm{t}=1 ; \mathrm{d}=1)$, para representação booleana e de freqüência.

\begin{tabular}{|c|c|c|c|c|c|c|c|}
\hline Class & $\begin{array}{c}\text { Reduçāo } \\
250\end{array}$ & $\begin{array}{c}\text { Reduçāo } \\
500\end{array}$ & $\begin{array}{c}\text { Reduçāo } \\
750\end{array}$ & $\begin{array}{c}\text { Reduçāo } \\
1.000\end{array}$ & $\begin{array}{c}\text { Redução } \\
1.500\end{array}$ & $\begin{array}{c}\text { Reduçäo } \\
\mathbf{3 . 0 0 0}\end{array}$ & $\begin{array}{c}\text { Sem } \\
\text { Redução }\end{array}$ \\
\hline $\begin{array}{l}\text { DCan_set } \\
\text { sVM-Bool } \\
\text { sVM-Freq }\end{array}$ & $\begin{array}{l}5,78 \pm 14,79 \\
7,03 \pm 14,77 \\
\end{array}$ & $\begin{array}{c}2,22 \pm 4,68 \\
4,58 \pm 7,91\end{array}$ & $\begin{array}{l}5,78 \pm 14,79 \\
8,28 \pm 14,64\end{array}$ & $\begin{array}{l}5,78 \pm 14,79 \\
8,28 \pm 14,64\end{array}$ & $\begin{array}{l}5,78 \pm 14,79 \\
5,78 \pm 14,64 \\
\end{array}$ & $\begin{array}{c}\mathbf{5 , 7 8} \pm \mathbf{1 4 , 7 9} \\
\mathbf{7 , 0 3} \pm 14,77\end{array}$ & $\begin{array}{c}6,11 \pm 15,81 \\
5,83 \pm 8,09 \\
\end{array}$ \\
\hline $\begin{array}{l}\text { DStem_set } \\
\text { SVM-Bool } \\
\text { SVM-Freq }\end{array}$ & $\begin{array}{l}7,85 \pm 9,32 \\
6,34 \pm 9,78\end{array}$ & $\begin{array}{l}7,14 \pm 9,65 \\
6,96 \pm 9,97\end{array}$ & $\begin{array}{l}7,14 \pm 9,65 \\
6,96 \pm 8,04\end{array}$ & $\begin{array}{l}7,14 \pm 9,75 \\
6,34 \pm 7,80\end{array}$ & $\begin{array}{l}6,34 \pm 9,32 \\
6,96 \pm 8,04\end{array}$ & $\begin{array}{l}6,34 \pm 9,32 \\
6,96 \pm 8,04\end{array}$ & $\begin{array}{l}7,05 \pm 9,52 \\
6,96 \pm 8,04 \\
\end{array}$ \\
\hline $\begin{array}{l}\text { NCan_set } \\
\text { SVM-Bool } \\
\text { SVM-Freq }\end{array}$ & $\begin{array}{c}10,27 \pm 8,78 \\
14,20 \pm 13,27\end{array}$ & $\begin{array}{c}7,14 \pm 7,99 \\
14,02 \pm 13,28\end{array}$ & $\begin{array}{c}9,11 \pm 7,75 \\
14,02 \pm 13,28\end{array}$ & $\begin{array}{c}9,20 \pm 7,89 \\
14,02 \pm 13,28\end{array}$ & $\begin{array}{l}11,88 \pm 11,93 \\
14,02 \pm 13,28\end{array}$ & - & $\begin{array}{c}7,14 \pm 8,51 \\
\mathbf{3 , 3 0} \pm \mathbf{4 , 5 7}\end{array}$ \\
\hline $\begin{array}{l}\text { NStem_set } \\
\text { SVM-Bool } \\
\text { SVM-Freq }\end{array}$ & $\begin{array}{c}6,43 \pm 6,60 \\
5,36 \pm 6,07\end{array}$ & $\begin{array}{l}5,93 \pm 4,68 \\
2,68 \pm 3,47\end{array}$ & $\begin{array}{c}5,89 \pm 4,67 \\
4,02 \pm 5,67\end{array}$ & $\begin{array}{c}5,89 \pm 4,67 \\
\mathbf{3 , 3 9} \pm \mathbf{4 , 9 2}\end{array}$ & $\begin{array}{c}5,89 \pm 4,67 \\
2,68 \pm 3,47\end{array}$ & - & $\begin{array}{c}4,68 \pm 3,24 \\
3,30 \pm 4,57\end{array}$ \\
\hline
\end{tabular}

Tabela 6.59: Erro total com algoritmo SVM, e as diferentes reduçōes de features

Analisando esses resultados pode-se chegar a algumas conclusões, além das que já foram feitas ao longo deste capítulo, nas seções anteriores.

Primeiramente foram analisados os 4 conjuntos de dados DCan_set, DStem_set, NCan_set e NStem_set para o SVM com parâmetros default $(\mathrm{t}=1 ; \mathrm{d}=1)$ para a representação booleana e de freqüência e comparando todos esses conjuntos em relação ao erro total, o menor resultado foi obtido utilizando o conjunto de dados DCan_set com representação booleana e redução para 500 features. Em seguida ficaram os conjuntos NCan_set e NStem_set sem redução de features, com representação de freqüência.

Analisando as reduções, pode-se observar que as reduções de 250, 750, $1.000 \mathrm{e}$ 1.500 features para os 3 conjuntos de dados DCan_set, DStem_set e NCan_set não obtiveram os melhores resultados.

Os melhores resultados para as reduções de $250,750,1.000,1.500$ e sem redução de features foram obtidos com o conjunto de dados NStem_set. Esse conjunto de dados usa a identificação de atributos mais simples, usando o método de stemming baseado no algoritmo de Porter e a técnica de Pré-processamento também a mais simples, baseada 
em (Weiss \& Indurkhya 1998), ou seja, todas as features são compostas de uma única palavra (1-gram). Neste conjunto de documentos essa representação gerou 1.956 features que também é o menor conjunto de features.

A aplicação das reduções não melhora em muito os resultados obtidos em relação ao erro total, sendo que para o conjunto de dados NCan_set o melhor resultado foi obtido sem redução de features com representação de freqüência. Para o conjunto de dados NStem_set, o resultado obtido sem redução de features com representação de freqüência ficou em segundo lugar. Para cada conjunto de dados há necessidade de se verificar qual a melhor redução para se conseguir um melhor desempenho quanto às taxas de erro. Portanto, nem sempre se aplicando reduçōes há uma melhora nas taxas de erro, como pode ser observado na Tabela 6.59.

$\mathrm{Na}$ Tabela 6.60 são mostrados os resultados dos 4 experimentos, utilizando os algoritmos da ferramenta $W e k a$, para representação booleana e de freqüência com a redução para 500 features.

Analisando os conjuntos de dados DCan_set e NStem_set para os algoritmos da ferramenta Weka o menor erro total foi obtido pelo algoritmo Tabela de Decisão com o conjunto de dados DCan_set com representação de freqüência. Em seguida ficou o algoritmo Regras de Decisão também com o conjunto de dados DCan_set com representação de freqüência. Ressalta-se que este valor é muito semelhante ao melhor valor obtido com SVM, também com o conjunto de dados DCan_set. O pior resultado ocorreu com Vizinho mais Próximo para o conjunto de dados NStem_set.

\subsection{Considerações Finais}

Os experimentos apresentaram uma tendência do que ocorre com outras línguas, como $\circ$ inglês e o chinês. SVM apresentou melhores resultados para a categorização de textos. Experimentos similares foram realizados por vários autores. (Joachims 1998) comparou 5 algoritmos de aprendizado, Naive Bayes, Rocchio, Vizinho mais Próximo, C4.5 e Support Vector Machines, para tratamento com textos em inglês. Ele constatou que, exceto SVM e Naive Bayes, o número ótimo de fetaures foi menor que o número total de features. Nos experimentos por ele realizado, para SVM, o uso de todas as features apresentou uma 


\begin{tabular}{|c|c|}
\hline \multicolumn{2}{|c|}{ Conjunto de Dados DCan_set } \\
\hline Classificador & $\begin{array}{c}\text { Reducāo } \\
500\end{array}$ \\
\hline $\begin{array}{c}\text { Nalve Bayes } \\
\text { Bool }\end{array}$ & $5,78 \pm 14,79$ \\
\hline $\begin{array}{c}\text { Naive Bayes } \\
\text { Freq }\end{array}$ & $13,10 \pm 14,51$ \\
\hline $\begin{array}{l}\text { Vizinho mals } \\
\text { Próximo-Bool }\end{array}$ & $5,78 \pm 14,79$ \\
\hline $\begin{array}{l}\text { Vizinho mals } \\
\text { Próximo-Freq }\end{array}$ & $5,56 \pm 14,10$ \\
\hline $\begin{array}{c}\text { Árvore } \\
\text { Decisāo-Bool }\end{array}$ & $7,21 \pm 14,85$ \\
\hline $\begin{array}{c}\text { Árvore } \\
\text { Declsāo-Freq }\end{array}$ & $8,23 \pm 14,07$ \\
\hline $\begin{array}{c}\text { Regras } \\
\text { Declsāo-Bool }\end{array}$ & $5,78 \pm 14,79$ \\
\hline $\begin{array}{c}\text { Regras } \\
\text { Decísäo-Freq }\end{array}$ & $3,47 \pm 5,60$ \\
\hline $\begin{array}{c}\text { Tabela } \\
\text { Declsão-Bool }\end{array}$ & $5,78 \pm 14,79$ \\
\hline $\begin{array}{c}\text { Tabela } \\
\text { Decisāo-Freq }\end{array}$ & $2,22 \pm 4,68$ \\
\hline
\end{tabular}

\begin{tabular}{|c|c|} 
Conjunto de Dados NStem.set \\
\begin{tabular}{|c|c|}
\hline Classificador & $\begin{array}{c}\text { Redução } \\
500\end{array}$ \\
\hline $\begin{array}{c}\text { Nalve Bayes } \\
\text { Bool }\end{array}$ & $10,57 \pm 6,93$ \\
\hline $\begin{array}{c}\text { Naive Bayes } \\
\text { Freq }\end{array}$ & $18,93 \pm 11,44$ \\
\hline $\begin{array}{c}\text { Vizinho mals } \\
\text { Próximo-Bool }\end{array}$ & $17,43 \pm 10,42$ \\
\hline $\begin{array}{c}\text { Vizinho mals } \\
\text { Próximo-Freq }\end{array}$ & $39,20 \pm 14,60$ \\
\hline $\begin{array}{c}\text { Arvore } \\
\text { Decisäo-Bool }\end{array}$ & $11,86 \pm 8,36$ \\
\hline $\begin{array}{c}\text { Árvore } \\
\text { Decisäo-Freq }\end{array}$ & $11,52 \pm 12,64$ \\
\hline $\begin{array}{c}\text { Regras } \\
\text { Decisão-Bool }\end{array}$ & $23,63 \pm 10,22$ \\
\hline $\begin{array}{c}\text { Regras } \\
\text { Decisäo-Freq }\end{array}$ & $22,32 \pm 12,77$ \\
\hline $\begin{array}{c}\text { Tabela } \\
\text { Decisäo-Bool }\end{array}$ & $7,98 \pm 9,30$ \\
\hline Tabela \\
Declsäo-Freq
\end{tabular} & $7,14 \pm 7,47$ \\
\hline
\end{tabular}

Tabela 6.60: Erro total com algoritmos da Weka, e as diferentes reduções de features

melhor eficiência do que para os outros algoritmos. Nos experimentos aqui realizados o Naive Bayes não teve um bom desempenho.

Com esse experimento, confirmou-se o que foi encontrado na literatura, em que SVM é superior a outros algoritmos de categorização de texto, como Vizinho mais Próximo, em (Cooley 1999) e (Joachims 1998). Como mostrado em (Cooley 1999), observou-se que não há necessidade de usar redução de features, em se tratando do algoritmo SVM. Neste trabalho também foi verificado que a diferança de desempenho em geral não foi tão significativa.

Os melhores resultados para quase todas as reduções foi para o conjunto de dados NStem_set, e este é o conjunto de dados mais simples. Esse conjunto de dados usa a identificação de atributos mais simples, o método de stemming baseado no algoritmo de Porter e a definição de Pré-processamento também a mais simples, baseada em (Weiss 
\& Indurkhya 1998). Essa representação gera 1.956 features que também é o conjunto de menor número de features para a mesma base de dados.

Os melhores resultados foi obtido pelo SVM com parâmetros default e para a Tabela de Decisão, ambos para o conjunto de dados DCan_set.

Há uma melhora, mas nem sempre muito significativa dos resultados quando se aplicam reduções ao conjunto de dados. Há necessidade de se pesquisar para se encontrar a melhor redução para cada conjunto.

O capítulo seguinte mostra as conclusões finais e os possíveis trabalhos futuros, em complemento a esse trabalho. 


\section{Capítulo 7}

\section{Conclusões}

Text Mining é uma área muito importante e seus domínios de aplicações são numerosos, principalmente devido a proliferação de documentos digitais, tais como bibliotecas digitas, Intranets e a própria Internet. Isso facilitou imensamente o rápido acesso a informações atualizadas sobre os mais diversos assuntos. Dentro dessa gama de informaçōes existe conhecimentos importantes inseridos nos documentos. O processo de Text Mining tornase importante por apoiar essa extração de conhecimento.

O processo de Text Mining pode ser dividido em quatro etapas: coleta de documentos, pré-processamento, extração de conhecimento e avaliação dos resultados. A etapa de coleta é coletar os documentos relacionados ao domínio da aplicação a ser avaliada, podendo ser coletados os mais diferentes tipos de documentos. A etapa de pré-processamento fornece a representação com a qual é possivel aplicar os algoritmos de extração de conhecimento. Para o desenvolvimento dessa etapa foi projetado e desenvolvido em (Imamura 2001) scripts em Perl para atribuição de pesos, necessários para a construção da representação, que é representada na forma de atributo-valor.

Para a construção dos scripts de identificação de atributos, neste trabalho foram utilizados como base 2 algoritmos distintos baseados em (Mladenić 1998) e (Weiss \& Indurkhya 1998). O algoritmo baseado em (Mladenić 1998) leva em consideração os atributos formados por até cinco termos que não sejam stopwords e que tenham ocorrido pelo menos quatro vezes nos documentos. No algoritmo baseado em (Weiss \& Indurkhya 1998) são identificados atributos formados por apenas um termo que não seja stopwords. 
Para cada um desses algoritmos foi considerado o uso de uma estratégia para a realização da normalização dos termos com base em stemming, utilizando o algoritmo de Porter e outra com base na análise léxica e sintática, provida de uma ferramenta, o etiquetador, feita pelo NILC.

A escolha desses scripts para a preparação dos documentos deste trabalho foi feita com base nos resultados apresentados nos experimentos descritos em (Imamura 2001).

$\mathrm{Na}$ etapa de extração de conhecimento foram aplicados algoritmos com o objetivo de classificar os textos existentes em classes bi-valoradas. Os algoritmos avaliados foram: Vizinho mais Próximo, Naive Bayes, Árvore de Decisão, Regras de Decisão, Tabelas de Decisão, implementados na ferramenta Weka, e Support Vector Machines, implementado na ferramenta $S V M^{\text {light }}$. Esses algoritmos foram escolhidos por terem sido mais citados na literatura, para a língua inglesa. Neste trabalho foi estudada basicamente a tarefa de categorização.

$\mathrm{Na}$ etapa de avaliação dos resultados foram apresentados os dados obtidos pelos algoritmos, utilizando-se das métricas de avaliação, tais como: erro total, erro da classe positiva, cobertura e $F_{1}$.

Como base de trabalho para testar os algoritmos contou-se com uma base textual envolvendo textos do domínio de Aquisição de Conhecimento e Redes Neurais. Os experimentos descritos neste trabalho tiveram como objetivo verificar a eficácia dos algoritmos utilizados com categorização de textos, utilizando textos em português.

Alguns dos objetivos foram atingidos na medida em que se mostrou a aplicação das diversas técnicas de Inteligência Artificial no processo de Text Mining.

Como ocorre com experimentos semelhantes para o inglês, o melhor classificador foi o SVM juntamente com o algoritmo de Tabela de Decisão para esse conjunto de documentos em português.

O conjunto de dados mais simples NStem_set usando a definição de Pré-processamento mais simples baseada em (Weiss \& Indurkhya 1998), e também a normalização de palavras mais simples, que é o método de stemming baseado no algoritmo de Porter (Porter 1980) foi o conjunto que em quase todas as reduções obteve melhor resultado usando SVM. Essa representação gerou 1.956 features que também é o conjunto de menor 
número de features para a base de documentos ${ }^{1}$.

Há uma melhora, mas nem sempre muito significativa dos resultados quando se aplicam reduções ao conjunto de dados. Há necessidade de realizar diferentees experimentos para encontrar a melhor redução para cada conjunto específico.

No que diz respeito ao pré-processamento há necessidade de um estudo tratando principalmente documentos com classes que não sejam simplesmente bi-valoradas, como o realizado por (Hsu \& Lin 2001) para a língua chinesa e (Lee, Lin, \& Wahba 2001) para o inglês.

Uma das limitações deste trabalho diz respeito ao uso de somente uma única base textual, bivalorada. No tocante a extração de conhecimento ainda há necessidade de se completar o estudo, para trabalhos futuros, como:

- Experimentos com outros domínios

- Utilização de outros algoritmos verificando se não poderiam trabalhar melhor com o português, além dos estudados neste trabalho.

- Investigação de outros algoritmos para extração de conhecimento de textos, principalmente utilizando-se da combinação de estimadores (classificadores), como foi sugerido em (Sebastiani 2002), usando-se comitês, também chamados ensembles cujo objetivo é melhorar a precisão dos estimadores individuais, aproveitando as características intrínsecas de cada um.

- Investigação dos outros métodos de redução de atributos, a fim de verificar como eles podem afetar o desempenho dos algoritmos de extração de conhecimento de textos em português.

- A utilização de outros scripts de atribuição dos pesos, além dos utilizados neste trabalho.

\footnotetext{
${ }^{1}$ Os documentos estão disponiveis em http://labic.icmc.sc.usp.br
} 


\section{Referências}

Aas, K. \& L. Eikvil (1999). Text categorization: a survey. Technical Report 941, http: //www.nr.no/research/samba/textmining.html.

Apté, C., F. Damerau, \& S. M. Weiss (1994a). Automated learning of decision rules for text categorization. In ACM SIGIR '94 - 17th Annual ACM Conference on Research and Development in Information Retrieval, Volume 12, Dublin Ireland, pp. 233-251.

Apté, C., F. Damerau, \& S. M. Weiss (1994b). Towards language independent automated learning of text categorization models. In ACM SIGIR '94 - 17th Annual $A C M$ Conference on Research and Development in Information Retrieval, Dublin Ireland.

Apté, C., F. Damerau, \& S. M. Weiss (1998). Text mining with decision rules and decision trees. In ACM SIGIR '9.8 - 21th Annual ACM Conference on Research and Development in Information Retrieval.

Apté, C. \& S. M. Weiss (1997). Data mining with decision rules and decision trees. In Future Generation Computer Systems, pp. 197-210.

Baeza-Yazte, R. \& B. Ribeiro-Neto (1999). Modern information retrieval. Addison Wesley Longman Publishing Company.

Baranauskas, J. A. \& M. C. Monard (2000). Reviewing some machine learning concepts and methods. Technical Report 102, ICMC-USP, ICMC-USP. ftp://ftp. icmc.sc. usp.br/pub/BIBLIOTECA/rel_tec/Rt_102.ps.zip.

Batista, G. E. A. P. A. (1997). Um ambiente de avaliação de aprendizado de máquina utilizando exemplos. Dissertação de Mestrado, ICMC-USP, São Carlos, SP. 
Belkin, N. J. \& W. B. Croft (1992). Information filtering and information retrieval: two sides of the same coin? . In ACM Communications, Volume 35, pp. 29-38.

Braga, A. P., A. C. P. L. F.. Carvalho, \& T. B. Ludermir (2000). Redes neurais artificiais: teoria e aplicações. Livros Técnicos e Científicos (LTC).

Breiman, L. (1996). Bagging predictos. In Machine learning, Volume 24, pp. 123-140.

Breiman, L., J. H. Friedman, R. A. Olshen, \& C. J. Stone (1984). Classification and regression trees.

Burges, C. J. C. (1998). A tutorial on support vector machines for pattern recognition. Technical report. http://citeseer.nj.nec.com/burges98tutorial.html.

Cheeseman, P., J. Kelly, M. Self, J. Stutz, W. Taylor, \& D. Freeman (1990). AutoClass: a bayesian classification system. In Readings in Machine Learing, Morgan Kaufmann, pp. 431-441.

Clark, P. \& R. Boswell (1991). Rule induction with CN2: some recent improvements. In Lecture Notes in Computer Science, Springer Verlag, pp. 151-163.

Clark, P. \& T. Niblett (1989). The CN2 induction algorithm. In Machine Learning, Volume 3, pp. 261-283.

Cohen, W. W. (1995a). Fast effective rule induction. In Twelfth International Conference on Machine Learning, San Francisco, CA, pp. 115-123. http://www. research. att. com/ ${ }^{\sim}$ wcohen.

Cohen, W. W. (1995b). Learning to classify english text with ILP methods. In Workshop on Inductive Logic Programming, Leuven. http://www.research.att.com/ wcohen.

Cohen, W. W. (1995c). Text categorization and relational learning. In 12th International Conference on Machine Learning, Lake Tahoe, California. http://ww . research. att . com $/{ }^{\sim}$ wcohen.

Cohen, W. W. \& Y. Singer (1996). Context-sensitive learning methods for text categorization. In SIGIR'96 : Nineteenth Annual International ACM SIGIR Conference on Research and Development in Information Retrieval, pp. 307-315. http: 
//www. research. att. com/ ${ }^{\sim}$ wcohen.

Cooley, R. (1999). Classification of news stories using support vector machines. In Sixteenth International Joint Conference on Artificial Intelligence Text 'Mining Workshop.

Cooper, W. S. (1995). Some inconsistencies and misnomers in probabilistic information retrieval. In ACM Transactions on Information Systems, Volume 13, pp. 100-111.

Cover, T. M. \& P. E. Hart (1967). Nearest neighbor pattern classification. In IEEE Transactions on Information Theory, Volume 13, pp. 21-27.

Craven, M., A. McCallum, D. DiPasquuo, T. Mitchell, D. Freitag, \& K. Nigam (1998). Learning to extract symbolic knowledge from the World Wide Web. In AAAI'98 : 15th National Conference on Artificial Intelligence, Pittsburgh. http: /www. research. att. com/ ${ }^{\sim}$ craven.

Craven, M. \& S. Slattery (1998). Combining statistical and relational methods for learning in hypertext domain. In ILP'98 : 8th International Conference on Inductive Logic Programming. http: /www. research. att.com/ craven.

Crestani, F., M. Lalmas, C. J. V. Rijsbergen, \& I. Campbell (1998). "Is this document relevant? ...probably": a survey of probabilistic models in information retrieval. In ACM Computing Surveys, Volume 30, pp. 528-552.

-Dagan, R. F. . I. (1995). Knowledge discovery in textual database (KDT). In First International Conference on Knowledge Discovery and Data Mining, Montreal, Canada, pp. $112-117$.

Drucker, H., D. Wu, \& V. N. Vapnik (1999). Support vector machines for spam categorization. In IEEE Transactions on Neural Networks, Volume 10, pp. 1048-1054.

Dumais, S. T., J. Platt, D. Heckerman, \& M. Sahami (1998). Inductive learning algorithms and representations for text categorization. In ACM-CIKM98, pp. 148-155.

Fayyad, U. M., G. P. Shapiro, \& P. Smyth (1996). From data mining to knowledge discovery: an overview. In AAAI Press, Menlo Park, CA, pp. 1-34.

Felix, L., S. O. Rezende, C. Y. Doi, M. de Paula, \& M. Romanato (1998). $\mathcal{M L C + +}$ 
biblioteca de aprendizado de máquina em $C++$. Technical report, ICMC-USP, $\mathrm{S}$ ftp://ftp.icmc.sc.usp.br/pub/BIBLIOTECA/reI_tec/Rt_72.ps.zip.

Gunn, S. R. (1998). Support vector machines for classification and regression. Technical report, University of Southampton, Intelligente System Research Group. http:// www. isis .ecs. solon. ac. uk/resources/svminfo/svm.pdf.

Hearst, M. A. (1999). Untangling text data mining. In 37th Annual Meeting of the Association for Computational Linguistics.

Hearst, M. A., B. Scholkopf, S. Dumais, E. Osuna, \& J. Platt (1998). Trends and controversies - support vector machines. In IEEE Intelligent Systems, Volume 13, pp. $18-28$.

Hsu, C. \& C. Lin (2001). A comparison on methods for multi-class support vector machines. In IEEE Transaction on Neural Networks.

Imamura, C. Y. M. (2001). Pré-processamento para extraçāo de conhecimento de bases textuais. Dissertação de Mestrado, ICMC-USP, Sāo Carlos, SP.

Joachims, T. (1997). A probabilistic analysis of the Rocchio algorithm with TFIDF for text categorization. In ICML97: 14th International Conference on Machine Learning, pp. 143-151. www-ai.informatik.uni-dortmund.de/PERSONAL/joachims. html.

Joachims, T. (1998). Text categorization with support vector machines: learning with many relevant features. In ECML'98 : Tenth European Conference on Machine Learning, pp. 137-142. www-ai.informatik.uni-dortmund.de/PERSONAL/ joachims . html.

J.Weston \& C. Watkins (1998). Multi-class support vector machines. Technical report.

Kecman, V. (2001). Learning and soft computing: support vector machines, neural networks and fuzzy logic models. London, England: A Bradford Book, Cambridge, Massachusetts.

Kohavi, R. (1994). $M \mathcal{L C}++$ : a machine learning library in $C++$. In IEEE Computter Society Press, pp. 740-743. 
Kohavi, R., D. Sommerfield, \& J. Dougherty (1996). Data mining using $\mathcal{M L C + + ; ~ a ~}$ machine learning library in $C++$. In Toals with $I A$, pp. 234-245. PISA

Lagus, K. (2000). Text mining with the WEBSOM. Tese de Doutorado, Department of Computer Science and Engineering, Helsinki University of Technology, Espoo, Finland.

Lee, Y., Y. Lin, \& G. Wahba (2001). Multicategory support vector machines. In 33rd Symposium on the Interface, Number 1043. http://citeseer.nj.nec.com/465375.html.

Lewis, D. D. (1998). Naive (Bayes) at Forty: the independence assumption in information retrieval. In ECML'98: Tenth European Conference on 'Machine Learning, pp. $4-15$.

Lewis, D. D. \& W. A. Gale (1994). A sequential algorithm for training text classifiers. In 7th Annual International ACM-SIGIR Conference on Research and Development in Information Retrieval, pp. 2-12.

Ling, C. X. \& H. Wang (1997). Computing optimal attribute weight setting for nearest neighbor algorithms. In Artificial intelligence review, pp. 255-272.

Matic, N., I. Guyon, J. Denker, \& V. Vapnik (1993). Writer adaptation for on-line handwritten character recognition. In IEEE Computer Society Press, pp. 187-191.

McAuliffe, T. L. \& A. A. Afifi (1984). Comparison of a nearest neighbor and other approaches to the detection of space-time clustering. In Computational Statistics and Data Analysis, Volume 2, pp. 125-142.

McCallum, A. K. \& L. D. Baker (1998). Distributional clustering of words for text classification. In SIGIR '98, Rio de Janeiro. ht.tp://www. cs . cmu. edu/〜mccallum.

McCallum, A. K., K. Nigam, \& J. Lafferty (1999). Using maximum entropy for text classification. In IJCAI'g9 Workshop on Information Filtering. http://www.cs. cmu .edu/ ${ }^{\sim m c c a l l u m}$.

Michalski, R. S., I. Bratko, \& M. Kubat (1998). Machine learning and data mining: methods and applications. England: John Wiley and Sons Ltd.

Mitchell, T. M. (1997). Machine learning. McGraw-Hill Series in Computer Science. 


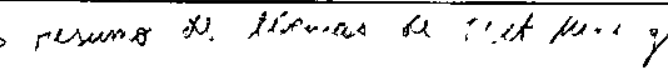

Dladenić, D. (1998). Machine learning on non-homogeneous, distributed text data. Tese de Doutorado, University of Ljubljana, Slovenia. http:/www-ai.ijs.si/ DunjaMladenic/home.html.

Murthy, S. K., S. Kasif, \& S. L. Salzberg (1994). A system for induction of oblique decision trees. In Journal of Artificial Intelligence Research, pp. 1-32.

Nilsson, N. J. (1996). Introduction to machine learning. In Stanford University.

Nunes, M. G. V. (1996). The design of a lexicon for brazilian portuguese: lessons learned and perspectives. In II. Workshop on Computational Processing of Written and Speak Portuguese, pp. 61-70.

cemesel

/Oliveira, R. B. T. \& S. O. Rezende (1998). Ferramentas de visualização de dados no MiniSet. Technical Report 71, ICMC-USP, ICMC-USP. ftp://ftp.icmc . sc . usp. br/pub/BIBLIOTECA/rel_tec/Rt_71.ps.zip.

Osuna, E., R. Freund, \& F. Girosi (1997). Training support vector machines: an application to face detection.

Padilha, T. P. P. (1999). Investigação de algoritmos de aprendizado de máquina pertencentes ao paradigma estatístico para aquisição de conhecimento. Dissertação de Mestrado, ICMC-USP, São Carlos, SP.

Pazzani, M. \& D. Billsus (1997). Learning and revising user profiles: the identification of interesting Web sites. In Machine Learning, Volume 27, pp. 313-331.

Pontil, M. \& A. Verri (1998). Support vector machines for 3d object recognition. In IEEE. Transactions on Pattern Analysis and Machine Intelligence, Volume 20, pp. 637-646.

Porter, M. (1980). An algorithm for suffixing stripping. In Program, Volume 14, pp. $130-137$.

Quinlan, J. (1993). C4.5: programs for machine learning. Los Altos, California: Morgan Kaufmann Publishers, Inc.

Quinlan, J. R. (1987). Generating production rules from decision trees. In Tenth International Joint Conference on Artificial Intelligence, Italy, pp. 304-307. 
Rezende, S. O., M. F. Paula, \& L. F. Figueiredo (1998). MiniSet ferramentas de data mining. Technical Report 80, ICMC-USP, $S$ ftp://ftp.icmc.sc.usp.br/pub/ BIBLIOTECA/rel_tec/Rt_80.ps.zip.

Rijsbergen, V. (1979). Information retrieval. London, Butterworths.

Roobaert, D. \& M. V. Hulle (1999). View-based 3D object recognition with support vector machines. In IEEE Neural Networks for Signal Processing Workshop, pp. $77-84$.

Rulequest-Research (1999). Data mining tools See5 and C5.0. http: //www . rulequest. com/see5-info.html.

Sebastiani, F. (2002). Machine learning in automated text categorization. $A C M$ Computing Surveys 34(1), 1-47. http:/faure.iei.pi.cnr.it/〜fabrizio/ Publications/ACMCS02 . pdf.

Tan, A. H. (1999). Text mining: the state of the art and the challenges. In Pacific Asia Conference on Knowledge Discovery and Data Mining: Workshop on Knowledge Discovery from Advanced Databases, pp. 65-70.

Tecuci, G. \& Y. Kodratoff (1995). Machine learning and knowledge acquisition: integrated approaches. In Academic Press.

Thomas, J. \& K. Sycara (1999). Integrating genetic algorithms and text learning for financial prediction. In GECCO-2000 Workshop on Data Mining with Evolutionary Algorithms.

Tkach, D. (1998). Text mining technology - turning information into knowledge. http: //www. software.ibm.com/data/iminer/fortext/download/whiteweb.html.

Vapnik, W. (1995). The nature of statistical learning theory. In Lecture Notes in Computer Science, Springer Verlag.

Weiss, S. M., C. Apté, , F. J. Damerau, D. E. Johnson, F. J. Oles, T. Goetz, \& T. Hampp (1999). Maximizing text mining performance. In IEEE Inteligent Systems, Volume 14, pp. 197-210. http://www. research.ibm.com/dar/papers/pdf/ ieee99_mtmp.pdf. 
Weiss, S. M., C. Apté, F. J. Damerau, D. E.Johnson, \& T. G. adn Thomas Hampp (1999). Maximizing text-mining performance. In IEEE Intelligent Information Retrieval, pp. 63-69.

Weiss, S. M. \& N. Indurkhya (1998). Predictive data mining - a practical guide. San Francisco, California: Morgan Kaufmann Publishers, Inc.

Willett, P. (1988). Recent trends in hierarchy Document clustering: a critical review. In Information Processing and Management, Volume 24, pp. 577-597.

Witten, I. H., G. W. Paynter, E. Frank, C. Gutwin, \& C. G. Nevill-Manning (1999). Kea: practical automatic keyphrase extraction. In $A C M D L$, pp. 254-255.

Yang, Y. (1999). An evaluation of statistical approaches to text categorization. In Journal of Information Retriveval Journal, pp. 69-90. http: / www . cs . cmu . edu/ “ yiming.

Yang, Y. \& X. Liu (1999). An re-examination of text categorization methods. In SIGIR '99 - 22nd ACM Internatinal Conference on Research and Development in Information Retrieval, Berkeley, US, pp. 42-49. http://www.cs. cmu . edu/ yiming.

Yang, Y. \& J. O. Pedersen (1997). A comparative study on feature selection in text categorization. In ICML '97 - 14th International Conference on 'Machine learning, pp. 412-420. http: /www.cs. cmu . edu/ ${ }^{\sim}$ yiming. 